Transactions of the Royal Society of Edinburgh

http://journals.cambridge.org/TRE

Additional services for Transactions of the Royal Society of Edinburgh:

Email alerts: $\underline{\text { Click here }}$

Subscriptions: $\underline{\text { Click here }}$

Commercial reprints: $\underline{\text { Click here }}$

Terms of use : $\underline{\text { Click here }}$

\title{
VIII.-An Analytical Theory of the Equilibrium of an Isotropic Elastic Plate
}

John Dougall

Transactions of the Royal Society of Edinburgh / Volume 41 / Issue 01 / January 1906, pp 129 - 228

DOI: $10.1017 /$ S0080456800033263, Published online: 06 July 2012

Link to this article: http://journals.cambridge.org/abstract_S0080456800033263

How to cite this article:

John Dougall (1906). VIII.-An Analytical Theory of the Equilibrium of an Isotropic Elastic Plate. Transactions of the Royal Society of Edinburgh, 41, pp 129-228 doi:10.1017/S0080456800033263

Request Permissions : $\underline{\text { Click here }}$ 


\section{VIII.-An Analytical Theory of the Equilibrium of an Isotropic Elastic Plate. By John Dougall, M.A. Commeniceteded lyy Professor (x. A. Grbson.}

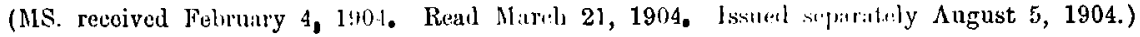

The following paper eoutains a purely inulytical discusion of the problem of the deformation of an isotropic elastic plate under wiven forces. The problem is an unusually interesting one. It was the first to be attacked (by L.IMI : all CLAPEYToN in 1828) after the istablishment of the general equations by NAviss. The solution of the problem of normal traction given by these authors, when rerlucerl to its simplest form, involves double integrals of simple harmonic functions of the coorlinates. The integrals are of complicated form, am practically impossible to interpret, a fact which, without doubt, has had much to do with the neglect of the problem in later times, and the almost complete absence of attempts to establish the approximate theory on the basis of an exact solution. An even more serious defect of Laní and Clapeyron's solution is that the integrals, as they stand, do not converge. I flaw of this sort has often been treated lightly by physical writers, the non-convergence of an integral being regarded as due to the inclusion of an infinite but unimportant constant. In the present case, however, the infinite terms are not constant, but functions of the coordinates, and the modifications necessary to secure convergence, so far from being unimportant, lead directly to the most significant terms of the solution.

The next writer to deal with the exact problem was Sir IV. THomson, who, at the end of the memoir in which he solved the problem of a spherical shell, indicated the form which the solution would take in the limiting case of a plate. His method, if carried out, would lead to integrals of the same form as Lame's.

Solutions of special problems have been given by other writers. Prof. Lavis hits wotkerl out the solution for an infinite solid subjected to normal pressure proportional to cos lice, and verified in this particular case some of the results of the approximate theory of thin plates (Proc. Lond. Wath. Soc., vol. xxi., 1859-90).

The history of the approximate theory is well known and easily accessible. It will be sutficient here to refer to-

(i) Todhunter and Pearson's History of the Elrsticity and Strength of Materials.

(ii) Clebsch's standard treatise, Theorie de l'elesticite des corps solides, as translated by Saint Venant ; in particular, Part I. chap. iii., aud SaInt Venant's brilliant note on $\$ 73$.

(iii) Prof. Love's Tiertise on the Theory of Elasticity, 189:- - especially the historical introductions to both volumes.

The various forms of the approximate theory rest partly upon the general equations of equilibrium, partly upon auxiliary hypotheses or physical principles. These principles are recognised as contained in the general equations, but on account of the

TRANS. ROY. SOC. EDIN, VOL. XLI. PART I. (NO. 8). 
analytical difficulties in the way of deducing them rignously, they are either simply assumed, or else supported by reasoning plausible rather than demonstrative.

In the following pages the problem is treated as a purely mathematical one, and the approximate theory for a finite plate deduced from an exact solution for an infinite plate. The main features of the method are-

(i) The use of Bessel functions in place of the simple harmonic functions of previous writers. Only the symmetrical forms, or functions of order zero, are required.

(ii) Transformation of the definite integrals, in terms of which the solutions are in the first place obtained, into series, by means of Cauchy's theory of contour integration and residues. The series involve Bessel functions of the second kind with complex arrament, and are so highly convergent that the principal features of the strain represented by the solution can be made out with the utmost ease. (The transformations belong to a class discussed systematically, apparently for the tirst time, in a paper "On the Determination of Green's Function by means of Cylindrical and Spherical Harmonics," Proc. Edin. Math. Soc. vol. xviii.)

(iii) Detailed solutions of the problems of internal force with vanishing face traction. The usual method of dealing with a general problem in Elasticity is to find a particular solution for the bodily force, and then to treat the problem of surface tractions completely. This is theoretically sufficient, but leaves the result in i complicaterl form, which in the present case must be simplified before practical applications can be made.

(iv) Use of Betti's Theorem (Love, Elrsticity, vol. i. \$140) to develop a method analogous to the method of Grecu's function in the Theory of the Potential, by which the properties of the solution for a finite plate can be rleduced from the infinite plate solution. (Cf. Proc. Edlin. Math. Soc., vol. xvi., "On a general Methor of Solving the Equations of Elasticity.")

The results of the ordinary theory are fully confirmed, and extended in various directions. The infinite solirl solution gires, of course, an exact particulir solution for internal force and traction on the plane fivces of a finite plate. It the hear of the solution appear the terms given by the approximate theory. In the rase of Hexure, the equation of Lagrange is obtained to :1 second approximation.

The problem of a finite plate under wiven enlge tractions cannot be completely solvenl, but exact solutions are given of certain problems relating to a circular plite. For a thin plate, with edge of any shine, the conditions satisfierl at the whe ly the principal terms of the exact solution are found to : degree of approximation beyond the reach of any theory which rests merely "n the "principle of the elastic cequivilence of statically cquipollent loads." For eximple, the celebrated boundary conditions given by Kireнног, in correction of Polsson, are verified, and extended by the inclusion of terms of higher order.

In conclusion, it may be mentioned that the methods riven here are cuually appliable to the problem of the vilsutions of a plate, and to the problems of the equilibrium and vibration of a finite circulitl cylinder, or of an open spherical shell. Some account of these applications I hope to publish shortly. 
TABLE OF CONTENTS.

INTRODUCTORY A.NAT,ISIS

(a) to $(g)$. Evaluation of definite integrals involving the linsil functions. Degenerate cases.

(h). Potential functions derived by integration from the point-son we potential. Definite integral expressions for these .

(i), (j). Solution of the problem of flow between two infinite parallel planes. Main stream and local currents . . . . . .

(k). Curvature. Diflerentiation as to are and normal

$\S 1$. Equations of equilibrium. Form of solution for a plate free from bodily force

$\S \supseteq$. Force applied at a single point of an infinite solid.

$\S 3$. Solution of the problem of normal traction for an infinite plate.

$\S 4$. Flexural and extensional components of the strain. Disadvantages of the solution in definite integrals

§5. Transformation of the definite integrals into series, by means of Cauchy's Theorem of contour integration

\$6. Types of the particular solutions composing the general solution

$\S 7$. Position of the zeroes of the functions sinh s.5.

\$8. Approximate forms of the $n^{\text {th }}$ terns of the infinite series, when $n$ is large.

\$9. The solution for arbitrary normal traction. Questions for discussion

$\$$ 10. Detailed solution of a special case. Term by term differentiations .

$\S 11$. The same special problem. Summation of two infinite series

$\S 12$. Final form of the same special solution.

$\$ 13$. Order of magnitude of the various parts of the solution when the thickness of the plate is small

\$14. Methods and results of the special case extended to the general problem of arbitrary normal traction.

$\$ 15$. Independent symbolical solution of the general problem.

$\S 16$. The problem of tangential face traction. Solution for an element of traction.

$\S 17$. Composition of the solution .

$\$ 18$. General solution. Comparison with the solution for normal traction

$\S 19$. Normal force applied at a single internal point. Solution in definite integrals.

$\S 20$. The same solution in serites.
Pran

$132 \$ 21$. Solution of a special problew of internal areal normal fricu: . . .

132 \$22. Approximate forms of displacements and stresses in the gerterin] case.

$\S$ 23. Normal force of constint, intensity throughout the thicknesim . . . . . . . 175

$\$ 4$. Internal for $:$ parallel to the faces . . 176

\$25. Solution . . . . . . . . . 178

136 26. Approximate iesults. Lagrange's equation

for flexure to a secisl approximation

$139 \$ 27$. Extensional strain. Differential equations of the principlal mode

$140 \$ 28$. Approximate values of the stresses across a plane parallel to the faces . . . . 182

142 \$29. Trunsmission of force to a distance. Expansions in polar coordinates . . . . 183

$113 \$ 30$. Typer of delormation conveying a given resultant stress . . . . . . 185

$\$ 31$. Conditions for the existence of a solution with finite potential energy. Elastic equivalence of statically equipollent loads.

$\$ 32$. Betti's reciprocal theorem. Verification of preceding solutions. . . . .

\$33. Finite plate unler edge tractions. Form of the solution deduced by means of Betti's Theorem . . . . . . . 19?

$\$ 34$. The same by another method . . . 195

35. General solution for an infinite solid under any forces. . . . . . . 196

$\$ 36$. Betti's Theorem and the problem of given elge tractions

$\$ 37$. Fxact solutions of special problems for a circular plate. Problem 1-symmetrical transverse displacement

\$38. Problem $2-$ normal displacement and normal shearing stress given. The Fourier and wher methods of obtaining such solutions.

\$39. Problem $:$ - permanent modes due to symmetrical edge tractions

\$40. Expansions of arbitrary functions..$\quad \cdot 206$

$\$ 41$. The problem of given edge tractions for a thin plate. . . . . . 208

42. Ext:nsio al strain . . . . . 208

$\$ 43$. The (ireen's function method for the permanent mode . . . . . . . . 213

163 $\$ \$ 44,45$. Flexural strain. Solutions to first and second approximation . . . 218, 220

$165 \S 46$. Flexural strain. The Green's function method. Kirchhoff's boundary conditions to a second approximation . . . 224

165

169 Addition to $P^{\prime}$ l'
$P_{A}+E$

701

73

76

180

] 81

82 


\section{INTRODUCTORY ANALYSIS.}

(a) The Bessel function $J$ is definer by the series

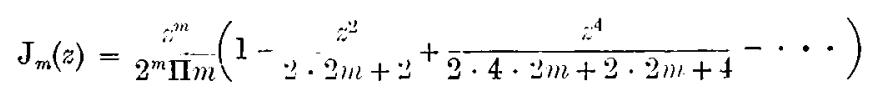

For the function of the second kind we take as definition

$$
\dot{C}_{m,}(:)=\frac{\pi}{2} \frac{J_{-m}(:)-e^{-2 m \pi} \mathbf{J}_{m,}(:)}{\sin m \pi}
$$

This makes $G_{t_{m}} \approx$ an analytic function of $m$, the value of which, when $m$ is a $r(m)$ integer, is

$$
\mathrm{G}_{m}(i)=\left(\operatorname{lom} 2-\gamma+\frac{\pi i}{2}\right), \mathrm{J}_{m}(i)-\mathrm{Y}_{i n}(z)
$$

where $Y_{m}(z)$ is Neumann's function.

In this case, therefore, $\mathrm{G}_{m} \approx=-\log \approx \Gamma_{m} \approx+$ a uniform function of $\approx$.

In the following pages we are concerned chiefly with the function of order zero

$$
\mathrm{G}_{0^{z}}=\left(\log 2-\gamma+\frac{\pi i}{2}\right)\left(1-\frac{z^{2}}{2^{2}}+\frac{z^{4}}{2^{2} \cdot 4^{2}}-\cdots\right)-\log z\left(1-\frac{z^{2}}{2^{2}}+\frac{z^{4}}{24^{2}}-\cdots\right)-\left(1+\frac{1}{2}\right)-4^{4}-\cdots
$$

When $\bmod :$ is very large, while the phase (argument) of $z$ lies between $-\pi / 2$ and $3 \pi / \approx$, then approximately

$$
\mathrm{G}_{m:=}=e^{-m \pi i} \stackrel{2}{2} e^{i\left(z+\frac{\pi}{4}\right)} \cdot \sqrt{\frac{\pi}{2}} .
$$

Similarly, when the phase of $z$ is between 0 and $\pi$ (excluding those values)

$$
\mathrm{J}_{1, \cdots}=e^{\frac{m-\pi i}{2}} e^{-i\left(2-\frac{\pi}{4}\right)} \sqrt{\frac{1}{2 \pi: i}} .
$$

(b) If $x, y, z$ and $\rho, \omega, z$ arr thr rectangular and cylindrical coordinates of a point in space, so that $x=\rho \cos \omega, y=\rho \sin \omega$, then the most important property of the Bessel Functions is that each of the eight functions

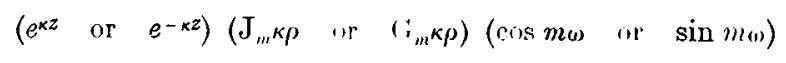

satisfies Laplace's equation, or in other words is a potential function.

Hence

Further, if

$$
\left(\nabla^{2}+\kappa^{2}\right) \cdot\left(J_{n} \kappa \rho \text { or } \quad\left(i_{m} \kappa \rho\right)(\cos m \omega \text { or } \sin m \omega)=0 .\right.
$$

then

$$
\begin{aligned}
\mathrm{R}^{2} & =\left(x-x^{\prime}\right)^{\prime 2}+\left(y-y^{\prime}\right)^{2} \\
& =\rho^{2}+\rho^{\prime \prime \prime}-2 \rho \rho^{\prime} \cos \left(\omega-\omega^{\prime}\right)
\end{aligned}
$$

$$
\left(\nabla^{2}+\kappa^{2}\right) \cdot\left(\mathrm{J}_{0} \kappa \mathrm{R} \text { or } \mathrm{G}_{0} \kappa \mathrm{R}\right)=0 .
$$

Let now $\mathrm{I}=\iint\left(\mathrm{r}_{0} \kappa \mathrm{R} f\left(x^{\prime}, y^{\prime}\right) d x^{\prime} d y^{\prime}\right.$, the integral being taken over a finite area $\mathrm{A}$.

Then $\left(\nabla^{2}+\kappa^{2}\right) \mathrm{I}=0$, if $(x, y)$ is without, 
but $\left(r^{2}+\kappa^{2}\right) \mathbf{I}=-2 \pi f\left(x^{2}, y\right)$, if $(., y)$ is within this :1lea; is ansily follows from the theorem

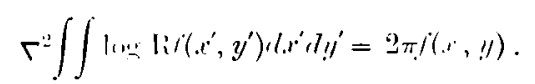

The differential equation sitisficl by I, together with the comlitions that I and its

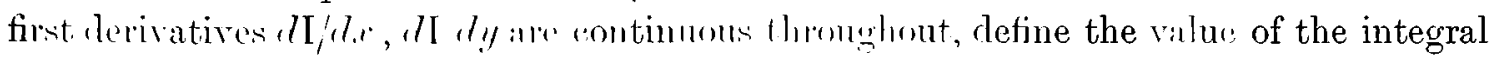
completely, and in many cases make its covaluation masy.

(c) For example, take $f^{\prime}\left(\iota^{\prime}, \prime^{\prime \prime}\right)=\mathrm{J}_{m} \beta \rho \cos m(1$, , with $m$ an integer, so that

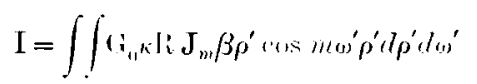

and suppose the area of integration to be a circle of $1:$ lius $"$, with centre at the origin. For convenience in the proof, let the imaginary part of $\kappa$ be positive.

$$
\begin{aligned}
& \text { Then } \mathrm{I}=\begin{array}{c}
2 \pi \\
\beta^{2}-\kappa^{2}
\end{array} \mathrm{I}_{m} \beta \rho \cos m \omega+\mathrm{AJ}_{m} \kappa \rho \cos m \omega, \text { when } \rho<a \text {, } \\
& =\mathrm{BG}_{m} \kappa \rho m m \omega, \quad \text {, when } \rho>a
\end{aligned}
$$

$A, B$ are determined from the conditions that $T$ and $d / / d \rho$ are continuous at $\rho=\alpha$ Thus we find

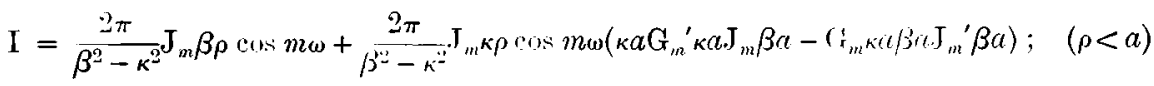

$$
\begin{aligned}
& =\frac{2 \pi}{\beta^{2}-\kappa^{2}} \mathrm{G}_{m} \kappa \rho \cos m \omega\left(\kappa+\mathrm{r}, \mathrm{J}_{m}{ }^{\prime} \kappa a \mathrm{~J} \mathrm{~J}_{m} \beta a-\mathrm{J}_{m^{\prime}} \kappa a \beta a \mathrm{~J}_{m}{ }^{\prime} \beta a\right) ; \quad(\rho>a)
\end{aligned}
$$

By the principle of continuation in the Theory of Functions, the result is true whitever loe the phase of $\kappa$. But when the phase of $\kappa$ is diminished loy $2 \pi$,

and

$$
\mathrm{G}_{m}\left(\kappa^{c}\right) \text { is increased by } 2 \pi i \mathrm{~J}_{m}\left(\kappa c^{c}\right)
$$

$$
\mathrm{O}_{m}{ }^{\prime}(\kappa c) \text { by } 2 \pi i J_{m}{ }^{\prime}(\kappa c) \text {; }
$$

hence, equating the corresponding changes in I and its value, we ubtain

$$
\begin{gathered}
\int_{0}^{a} \int_{0}^{2 \pi} \mathrm{J}_{0} \kappa \mathrm{li} \mathrm{J}_{m} \beta \rho^{\prime} \cos m \omega^{\prime} \rho^{\prime} \lambda p^{\prime} / l \omega^{\prime} \\
=\stackrel{2 \pi}{\beta^{\prime 2}-\kappa^{\prime 2}} \mathrm{~J}_{m} \kappa \rho \cos m \omega\left(\kappa a \mathrm{~J}_{m 2}{ }^{\prime} \kappa a \mathrm{~J}_{m} \beta a-\mathrm{J}_{m} \kappa a \beta a \mathrm{~J} \mathrm{~J}_{m}{ }^{\prime} \beta^{\prime} a\right) .
\end{gathered}
$$

From this again it easily follows that in $I$ and its value we may replace the $G$ functions by the $\mathrm{Y}$ functions.

(d) We have

$$
\begin{aligned}
& Y_{0} \kappa \rho=1 \ln \kappa \kappa \rho \mathrm{J}_{0} \kappa \rho+\frac{1}{4} \kappa^{2} \rho^{2}-\ldots . \\
& =\log \kappa \mathrm{J}_{0 \kappa \rho}+\log \rho\left(1-\frac{1}{4} \kappa^{2} \rho^{2} \ldots\right)+{ }_{1}^{2} \kappa^{\prime \prime} \rho^{2} . .
\end{aligned}
$$

Thus $\log \kappa \cdot J_{0} \kappa \rho-\mathrm{Y}_{0} \kappa \rho$ is an integral function of $\kappa$, in which

$$
\begin{array}{rlll}
\text { coefficient of } & \kappa^{0} & \text { is } & -\operatorname{lng} \rho, \\
\text { and coefficient of } & \kappa^{2} & \text { is } & \frac{1}{2} \mu^{2} \operatorname{lng} \rho-\frac{1}{4} \rho^{2} .
\end{array}
$$


The functions $\log \rho$ and $\frac{1}{4} \rho^{2} \log \rho-\frac{1}{4} \rho^{2}$ are thus in a sense degenerate forms of the Bessel Functions, and any theorem relating to the $\mathrm{G}$ or $\mathrm{Y}$ functions will yield a corresponcling theorem in these.

Thus by equating coefficients of $\kappa^{2}$ in the equation

we obtain

$$
\left(r^{*}+\kappa^{2}\right)\left(\log \kappa \mathrm{J}_{0} \kappa \rho-\mathrm{Y}_{0} \kappa \rho\right)=0
$$

and therefore

$$
\begin{aligned}
& \Gamma^{2} \log \rho=0 \\
& \Gamma^{2}\left(\frac{1}{4} \rho^{2} \log \rho-\frac{1}{4} \rho^{2}\right)=\log \rho
\end{aligned}
$$

We derluce at once

$$
\nabla^{4}\left(\frac{1}{4} \rho^{2} \log \rho-\frac{1}{4} \rho^{2}\right)=0 \text {. }
$$

$$
\begin{aligned}
\nabla^{2} \iint\left(\frac{1}{4} \mathrm{R}^{2} \log \mathrm{R}-\frac{1}{4} \mathrm{R}^{2}\right) f\left(x^{\prime}, y^{\prime}\right) d x^{\prime} d y^{\prime} & =\iint \log \mathrm{R} f\left(x^{\prime}, l^{\prime}\right) d l^{\prime} d l^{\prime} \\
\nabla^{4} \iint\left({ }_{4}^{1} \mathrm{R}^{2} \log \mathrm{R}-\frac{1}{4} \mathrm{R}^{2}\right) f\left(x^{\prime}, y^{\prime}\right) d x^{\prime} d y^{\prime} & =\nabla^{2} \iint \log \mathrm{R} f\left(x^{\prime}, y^{\prime}\right) d x^{\prime} d y^{\prime} \\
& ={ }^{\prime} \pi f(x, y) .
\end{aligned}
$$

and

(e) Again, from the addition theorem

we deduce

$$
\mathrm{Y}_{0} \mathrm{R}=\mathrm{Y}_{0^{\prime} \rho} \mathrm{J}_{0} \kappa \rho^{\prime}+2 \sum_{m=1} \mathrm{Y}_{m} \kappa \rho \mathrm{J}_{t n} \kappa \rho^{\prime} \cos m\left(\omega-\omega^{\prime}\right) ; \quad\left(\rho>\rho^{\prime}\right)
$$

and

$$
\log \mathrm{R}=\log \rho-\sum_{m=1} \frac{1}{\hbar}\left(\frac{\rho^{\prime}}{\rho}\right)^{m} \cos m\left(\omega-\omega^{\prime}\right) ; \rho>\rho^{\prime}
$$

$$
\begin{gathered}
\operatorname{li}_{4} \mathrm{R}^{2} \log \mathrm{R}-\frac{1}{4} \mathrm{R}^{2}=\left(\frac{1}{4} \rho^{2} \log \rho-\frac{1}{4} \rho^{2}\right)+4_{4}^{1} \rho^{\prime \prime 2} \log \rho \\
+\left\{(\rho-2 \rho \log \rho) \frac{\rho^{\prime}}{4}-\frac{\rho^{\prime 3}}{8 \rho}\right\} \cos \left(\omega-\omega^{\prime}\right) \\
+\sum_{m=2} \frac{1}{4 m}\left(\frac{\rho^{\prime}}{\rho}\right)^{m}\left(\frac{\rho^{2}}{m-1}-\frac{\rho^{\prime 2}}{m+1}\right) \cos m\left(\omega-\omega^{\prime}\right): \quad\left(\rho>\rho^{\prime}\right) .
\end{gathered}
$$

(f) In the same way, from the results of $(c)$, we may deduce the value of the integral

$$
I_{0}=\int_{0}^{a} \int_{0}^{\pi \pi}\left(\frac{1}{4} R^{2} \log R-\frac{1}{4} R^{2}\right) J_{m} \beta \rho^{\prime} \cos m \omega^{\prime} \rho^{\prime} d \rho^{\prime} d \omega^{\prime} .
$$

'The form of the result varies in the cases $m=0, m=1, m>1$. $m=0$ :

$$
\begin{aligned}
& 1_{0}=\stackrel{2 \pi}{\beta^{4}} J_{0} \beta \rho+\frac{\ddot{2} \pi}{\beta^{2}}\left\{\left(\frac{1}{\beta^{22}}-\frac{\rho^{2}}{4}\right)\left(\log a \beta a J_{0}^{\prime} \beta a-J_{0} \beta a\right)\right. \\
& \left.+\frac{a^{2}}{4}(1-\log a) \beta a \mathrm{~J}_{0}^{\prime} \beta a+{ }_{4}^{c^{2}}(\because \log a-1) \mathrm{J}_{0} \beta a\right\}, \quad \rho<a \\
& =\frac{2 \pi}{\beta^{2}}\left\{\left({ }_{4}^{1} \rho^{2} \log \rho-\frac{1}{4} \rho^{2}\right)\left(-\beta a \mathrm{~J}^{\prime} \beta a\right)+\log \rho\left(\frac{1}{\beta^{2}} \overline{-a^{2}}-\beta a \mathrm{~J}_{0}^{\prime} \beta a+\frac{a^{2}}{2} \mathrm{~J}_{0} \beta a\right)\right\}, \quad \rho>a \text {. } \\
& m=1 \text { : }
\end{aligned}
$$

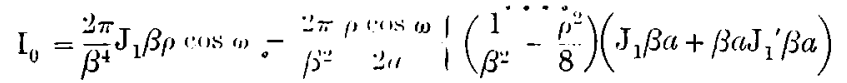

$$
\begin{aligned}
& \left.+{ }_{4}^{\prime 2}(1+2 \ln g a) \cdot \mathrm{J}_{1} \beta a+\frac{a^{2}}{4}\left(1-2 \ln \log _{5} a\right) \beta a \mathrm{~J}_{1}^{\prime} \beta a\right\}, \quad \rho<a \\
& =\frac{2 \pi}{\beta^{2}} \frac{a \cos \omega}{2 \rho}\left\{\left(\frac{1}{\beta^{2}} \cdots \frac{1}{4} \rho^{2}-\frac{1}{3} \rho^{\prime \prime} \log \rho\right)\left(\mathrm{J}_{1} \beta a-\beta a \mathrm{~J}_{1}^{\prime} \beta a\right)+\frac{a^{2}}{8}\left(\beta a \mathrm{~J}_{1}^{\prime} \beta a-3 \mathrm{~J}_{1} \beta a\right)\right\}, \quad \rho>a \text {. }
\end{aligned}
$$


$m>1$ :

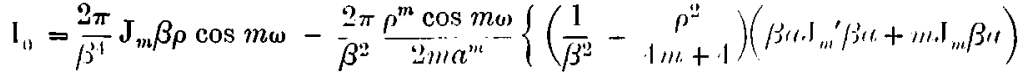

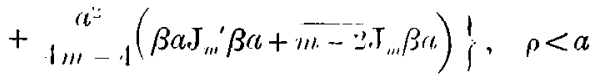

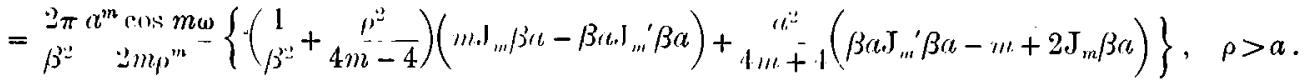

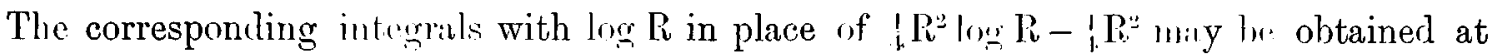
once by taking $\nabla^{*}$ of the above.

Also, all through we may write $\log (\mathrm{R} / c), \log \left(\rho^{\prime} c\right), \log (\alpha / c)$, instead of $\log \mathrm{R}, \log \rho$, $\log r$, this amounting merely to a change in the unit of length.

(g) By equating coefficients of like powers of $\beta$ in the results of $(c),(f)$ we can obtain

$$
\begin{aligned}
& \quad \int_{0}^{a} \int_{0}^{-\pi} \mathrm{G}_{0} \kappa \mathrm{R} \rho^{\prime m+2 n} \cos m \omega^{\prime} \rho^{\prime}\left(d \rho^{\prime} d(\omega)\right. \\
& \text { and } \quad \int_{0}^{a} \int_{0}^{2 \pi}\left(\frac{1}{4} \mathrm{R}^{2} \log \mathrm{R}-\frac{1}{4} \mathrm{R}^{2}\right) \rho^{\prime \prime \prime+2 n} \cos m \omega^{\prime} \rho^{\prime} d \rho^{\prime} d l \omega^{\prime} .
\end{aligned}
$$

In the case when $m=n=0$

$$
\begin{aligned}
& \int_{0}^{a} \int_{0}^{2 \pi} G_{0} \kappa \mathrm{R} \rho^{\prime} d \rho^{\prime} d \omega^{\prime}=-\frac{2 \pi}{\kappa^{2}}-{ }_{\kappa^{\prime}}^{2 \pi} J_{0} \kappa \rho \kappa a \mathrm{i}_{\mathrm{i}^{\prime},}{ }^{\prime} \kappa a, \rho<a \\
& =-\frac{2}{\kappa^{2}} \mathrm{G}_{0} \kappa \rho \kappa a \mathrm{~J}_{0}^{\prime} \kappa^{\prime \prime \prime}, \rho>a \text {. }
\end{aligned}
$$

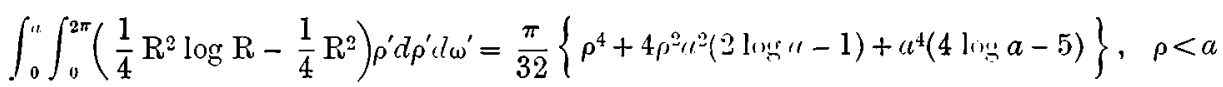

$$
\begin{aligned}
& =\left(\frac{1}{4} \rho^{2} \log \rho-\frac{1}{4} \rho^{2}\right) \pi c^{2}+\log \rho \frac{\pi a^{4}}{8}, \rho>a .
\end{aligned}
$$

These results and those of $(f)$ may easily be verified, or obtained, from the values of $\nabla^{4}$ of the integral, with the conditions that $I, \frac{d I}{d \rho}, \nabla^{2} \mathrm{I}, \frac{d}{d \rho} \nabla^{2} \mathrm{I}$ are continuous at $\rho=u$.

(h) In certain problems a class of potential functions occurs, which may be cleduced from the fundamental potential $1 / r$, where $r^{2}=x^{2}+y^{2}+z^{2}$, by successive integration with respect to $\approx$.

I'riting

$$
\begin{aligned}
& u_{1}=\log (r+z) \\
& u_{2}=z \log (r+z)-r \\
& u_{3}=\frac{1}{2}\left(z^{2}-\frac{1}{2} \rho^{2}\right) \log (r+\cdots)-\frac{3}{4} r z+\frac{1}{4} \rho^{2}
\end{aligned}
$$

we may easily verify that $u_{1}, u_{2}, u_{3}$ are potential functions, and that

$$
\begin{aligned}
& \frac{d u_{3}}{d:}=u \\
& d u_{2}=u_{1} \\
& d:=\frac{d u_{1}}{d z}=\frac{1}{r}
\end{aligned}
$$


These $z$-integrals of $1 / r$ may be expressed in the form of definite integrals involving the Bessel function $\mathrm{J}$, analogous to the integral forms for $r^{-1}$ and its $z$-derivatives,

$$
\begin{aligned}
r^{-1} & =\int_{0}^{\infty} e^{-\kappa z J_{0} \kappa \rho d \kappa} \\
\frac{d}{d z} r^{-1} & =\int_{0}^{\infty}(-\kappa) e^{-\kappa z} J_{0} \kappa \rho d \kappa, \text { etc., where } z>0 .
\end{aligned}
$$

We may notice that the value of $\int_{0}^{\infty} e^{-\kappa z} \mathrm{~J}_{U^{\kappa}} \rho d k$ follows at once from the remark that it is a potential symmetrical about the axis of $z$, and taking on that axis the value $\int_{0}^{\infty} e^{-\kappa^{z}} d \kappa=\frac{1}{z}$. We may use this idea to express $u_{1}, u_{2}, u_{3}$ in similar form.

For we have

and

$$
\int_{11}^{\kappa}(e-\kappa z-p-\kappa c) \frac{d \kappa}{\kappa}=-\log \frac{z}{c}
$$

$$
\begin{aligned}
& \int_{0}^{\infty}\left(e^{-\kappa z}-1+\kappa z e^{-\kappa c}\right) \frac{d \kappa}{\kappa^{2}}=z \log \frac{z}{c}-z \\
& \int_{0}^{x}\left(e^{-\kappa z}-1+\kappa:-\frac{1}{2} \kappa^{2} z^{2} e^{-\kappa c}\right) \frac{d \kappa}{\kappa^{3}}=-\frac{1}{2} \hat{2}^{2} \log \frac{z}{c}+\frac{3}{4} \cdots^{2},
\end{aligned}
$$

by integration with respect to $\approx$ from 0 to $z$.

Hence

$$
\begin{aligned}
& \int_{0}^{\infty}\left(e^{-\kappa z \mathrm{~J}}{ }_{0} \kappa \rho-e^{-\kappa c}\right) \frac{i \kappa}{\kappa} \quad=-\log \stackrel{r+z}{2+z} \\
& \left.\int_{0}^{\infty}\left(e^{-\kappa z J} J_{0} \kappa \rho-1+\kappa z e^{-\kappa c}\right)\right)_{\kappa^{2}}^{d k} \quad=z \log \frac{r+z}{2 c}-r
\end{aligned}
$$

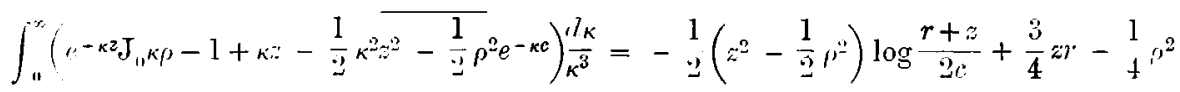

because in each case the functions equated are symmetrical potentials, taking the same value on the axis of symmetry.

By putting $z=0$ in the first and third of these we obtain two integrals, of great importance in the following analysis,

$$
\begin{aligned}
& \int_{1}^{\infty}\left(J_{0} \kappa \rho-e^{-\kappa c}\right) \frac{d \kappa}{\kappa}=-\log \frac{\rho}{2 c}
\end{aligned}
$$

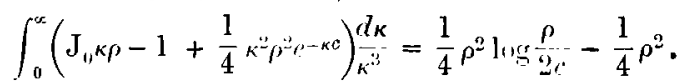

There is no difficulty in generalising the above results, but those given are all that we shall require.

(i) With a view to indicating the broul lines of the treatment of the elastic problem given in the succeeding pages, a liscussion on similar lines may be given here of a simple problem in potential, in which the attention is not distracted from the principles of the method by any complexity in the calculations.

The problem is to find the flow from a source situated between two parallel planes $z= \pm h$, under the condition that there is no flow across these planes. 
$W_{1}$ require a potential $V$, Ixeoming infunite ass $1 / 1$ at $\left(. r^{\prime}, y^{\prime}, z^{\prime}\right)$, but with no other singularity at a finite distance, and such that $\frac{d V}{d}=0$ when $\because= \pm h$.

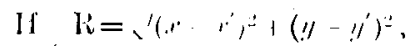

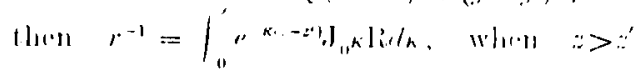

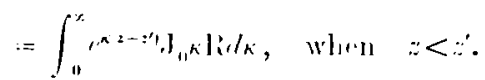

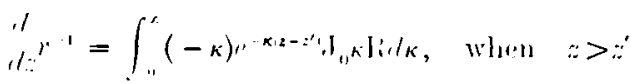

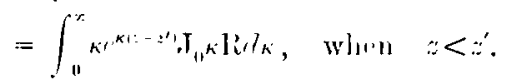

We therefore begin by finding : potentiil

giving

$$
\mathrm{Y}_{1}=(A \cosh \kappa \cdot i+\mathrm{Binh} \kappa) \cdot \mathrm{J}_{0} \kappa \mathrm{R}
$$

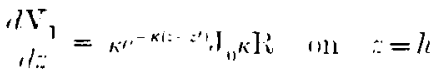

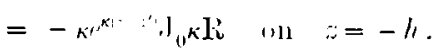

We obtain

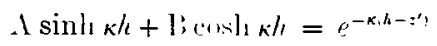

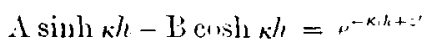

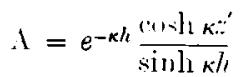

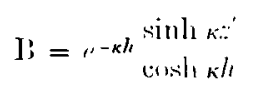

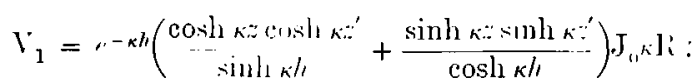

If this could be integrated with respert to is from 0 ti $x_{\text {s }}$, we should have a potential just balancing at the boundary the flow from the source.

But $V_{1}$ becomes infinite as $1 / \kappa h$ at $\kappa=0$, and the integration cannot be performed. We may, however, subtract from $V_{1}$ the (constant) potentill $e^{-\kappa c / k} /$, where $c$ is an arbitrary positive quantity. This makes interration possible, without introducing any flow across the boundary.

A solution of the problem is then

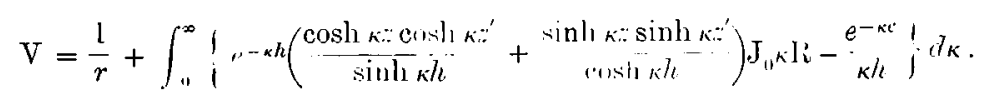

But this form of solution, while theoreticilly complete, is of little ralue because of the difficulty of interpretation. For example, it wives no indication of what on phrsical grounds we should expest to be the chief fiature of the phenomenon, namely, the practically two-dimensional chanater of the How at a morlerate distance from the source.

The transformation to which we procenl brings this out as luminously as possible.

First, it is convenient to reparite $V_{1}$ into its odd and even parts in $\kappa$, as is easily done by writing $\cosh \kappa h-\sinh \kappa / h$ for $e^{-\kappa h}$.

TRANS. ROY. SOC. EDIN., VOL. XLI. PART I. (NO. \&). 
Thus

$$
T_{1}=\left(-\cosh \kappa \overline{z-z^{\prime}}+\frac{\cosh \kappa h}{\sinh \kappa h} \cosh \kappa: z \cosh \kappa z^{\prime}-\frac{\sinh \kappa h}{\cosh \kappa h} \sinh \kappa * \sinh \kappa z^{\prime}\right) J_{0} \kappa \mathrm{R}
$$

Next we replace the term $1 / r$ in $\mathrm{V}$ by the equivalent integral

Hence

$$
\int_{0}^{\infty} e^{\mp \kappa\left(i-z^{\prime}\right)} \mathrm{J}_{0^{\kappa}} \mathrm{R} d \kappa
$$

$$
\left.\mathrm{V}=\int_{11}^{\infty},\left(\mp \sinh \kappa \overline{z-z}+\frac{\cosh \kappa h}{\sinh \kappa \bar{h}} \cosh \kappa z \cosh \kappa z^{\prime}-\frac{\sinh \kappa h}{\cosh \kappa h} \sinh \kappa: z \sinh \kappa z^{\prime}\right) \mathrm{J}_{0^{\circ}} \kappa \mathrm{R}-\frac{e-\kappa c}{\kappa h}\right\} d \kappa,
$$

the upper or lower sign being taken in the ambiguous term according as $z$ is $>$ or $<z^{\prime}$.

When $\mathrm{R}>0$, this integral can be separated into the two

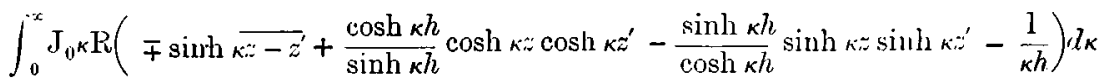

$$
\begin{aligned}
& +\int_{0}^{\infty}\left(\mathrm{J}_{0} \kappa \mathrm{R}-e^{-\kappa c}\right) \frac{d \kappa}{\kappa h} \text {. }
\end{aligned}
$$

The value of the latter integral we have found to be $-\frac{1}{h} \log \frac{k}{2 c}$.

The former integral is of the form $\int_{0}^{\infty} J_{11} \kappa \mathrm{RF}(\kappa) d \kappa$, where $\mathrm{F}(\kappa)$ is an orld function of $\kappa$, vanishing for $\kappa=0$. It may be expressed as a complex integral

$$
\frac{1}{\pi i} \int \mathrm{G}_{0} \kappa \mathrm{RF}(\kappa) d \kappa
$$

the path being from west to east along the whole of the real axis in the $\kappa$ plane, for

$$
\mathrm{G}_{0}(\kappa \mathrm{R})-\mathrm{G}_{0,}(\kappa ; \cdots \mathrm{R})=\pi i \mathrm{~J}_{0} \kappa \mathrm{R} \text {. }
$$

Now, from the original form of $V_{1}$, and the integral forms of $1 / r$, it is obvious that $\mathrm{F}(\kappa)$ vanishes at infinity in the eastern half of the $\kappa$ plane; being odd in $\kappa$ it must vanish likewise in the western half.

Hence by Cauchy's Theorem, the integral $\frac{1}{\pi i} \int \mathrm{G}_{0} \kappa \mathrm{RF}(\kappa) d \kappa$ is equal to twice the sum of the residues of the function $\left(x_{0} \kappa \mathrm{RF}(\kappa)\right.$ at its poles in the upper half of the $\kappa$ plane, and

$$
\begin{aligned}
& \mathrm{V}=-\frac{1}{h} \log _{5} \frac{\mathrm{R}}{2 c} \\
& +\frac{2}{h} \sum_{n=1} \cos \frac{n \pi \pi}{h} \cos \frac{n \pi)^{\prime}}{h}\left(i_{i} \frac{i n \pi \mathrm{r}}{h}\right. \\
& +\frac{2}{h} \sum_{n=0} \sin \left(n+\frac{1}{2}\right) \frac{\pi z}{h} \sin \left(n+\frac{1}{\bar{z}}\right) \frac{\pi z^{\prime}}{h} \mathrm{G}_{0}\left(n+\frac{1}{2}\right) \frac{i \pi \mathrm{R}}{h} .
\end{aligned}
$$

(j) The solution indicates (i) a main current in two dimensions, lefined by the

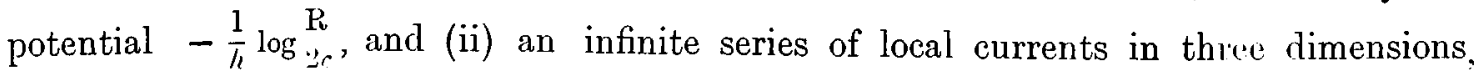


practically insensille when the distance from the somes is a morterate multiple of the thickness of the plate. In the following pinges we shall derluce analogrous solutions for 'sources of strain' of the dillirent types which may exist in an elastic solid, and develop

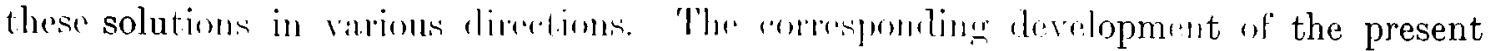
solution is extremely assy, but would carry us fow lits. Wo morely mention that the

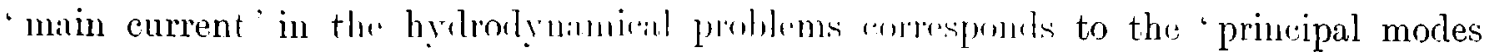
of strain, the determination of which is the aljeret of the theory of thin plates. But there is one imprortant distimetion in the two rases. In the flow problems the exact conditions definimg the 'main current' man always be found, and are indeed obvions: on the other hand, the and wous conditions in the strain problems can only be found by approximation.

(k) The following conventions seem to be very generally inlopted, but to prevent any risk of ambiguity they may le stated explicitly here. Consider any continuous plane area $A$ bounded externally by a closed curve $C_{-11}$, and internally ly one or more

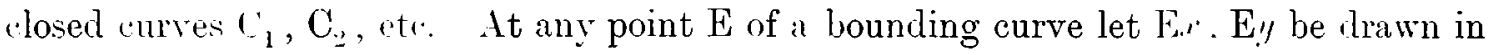
the directions of the rectangular ases of coorlinates. Let E $a$. Ey be turned through an angle $\epsilon$, which will be taken as positive when the rotation is counter-clockwise, until they coincide with $\mathrm{E} \xi, \mathrm{E} \eta$, the lirection of $\mathrm{E} \xi$ being that of the normal at $\mathrm{E}$ when drawn from within A towarls the boundary. $\mathrm{E} \xi$, $\mathrm{E} \eta$ will be taken to be the positive directions of the normal and tangent at $\mathrm{E}$, anrl if $f^{\circ}(x, y)$ be any function given within $\Lambda, \frac{d f}{d n}$ and $\frac{d f}{d s}$ will be usel to denote the rates of variation of $f$ per unit length in these positive directions.

The curvature at $\mathrm{E}$ is $\frac{d^{\prime}}{d s}$ and is denoted by $1 / \mu . \quad \rho$ is therefore positive when, in order to reach the centre of curvature from $\mathrm{E}$, we have to proceed into the area $A$.

If we suppose the figure traced on level ground, i person proceeding along the boundary in the positive direction will have the area on his left, and the curvature will be positive when he is rotating about the vertical in the counter-clockwise sense.

The following formule relating to differentiation along the are and normal will be much used in the later sections of the paper. Suppose the axes of $r$ and "! to coincide with the positive normal and tangent at a point ") of the bounding curve. It a neighbouring point $\mathrm{E}(r, y)$ on this curre

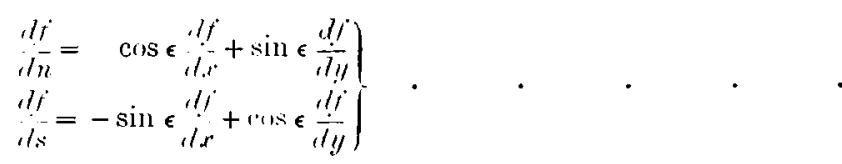

By putting $a, y, \epsilon$ equal to zero, we have at "

$$
\frac{d f}{d n}=\frac{d f}{d, r} ; \frac{d i}{d x}=\frac{d f}{d !} \quad . \quad . \quad . \quad . \quad .
$$


Differentiate the first of equations (i) with respect to $s$. Thus

$$
\begin{aligned}
& \frac{d}{d s}\left(\frac{d f}{d n}\right)=\cos \epsilon \frac{d l}{d s}\left(\frac{d f}{d x}\right)+\sin \epsilon \frac{d}{d s}\left(\frac{d f}{d y}\right)-\sin \epsilon \frac{d \epsilon}{d s} \frac{d f}{d x}+\cos \epsilon \frac{d \epsilon}{d s} \frac{d f}{d y} \\
& =\cos \epsilon\left(-\sin \epsilon \frac{\lambda^{2} f}{d x^{2}}+\cos \epsilon \frac{d^{y} f}{d x d y}\right)+\text { etc. } \\
& \text { and at } 0, \quad \frac{d}{d s} \frac{d f}{d n}=\frac{d r^{2}}{d x d y}+\frac{d \epsilon}{d s} \frac{d f}{d y} \\
& \text { or } \quad \frac{d^{2} f}{d x d y}=\frac{d}{d s} \frac{d f}{d n}-\frac{1}{\rho} \frac{d f}{d l s}
\end{aligned}
$$

Similarly from the second of (i),

$$
\begin{aligned}
& \text { at } \mathrm{O}, \frac{d^{2} f}{d s^{2}}=\frac{l^{2} f}{d y^{2}}-\frac{1}{\rho} \frac{d f}{d x} \\
& \text { or } \frac{d^{2} f}{d y^{2}}=\frac{d^{2} f}{d s^{2}}+\frac{1}{\rho} \frac{d f}{d x}
\end{aligned}
$$

Thus the values at $O$ of $\frac{d f}{d x}, \frac{d f}{d y}, \frac{d^{2} f}{d x d y}, \frac{d^{2} f}{d y^{2}}$ are known when $f$ and $\frac{d f}{d n}$ are given along the boundary.

$\frac{d^{2} f}{d x^{2}}+\frac{d^{2} f}{d y^{2}}$ or $\nabla^{2} f$ being an invariant for all systems of rectangular axes, we may also conveniently take

$$
\frac{l^{2} f}{d n^{2}}=\Gamma^{2} f-\frac{1}{\rho} \frac{d f}{d n}-\frac{d l^{2} f}{d s^{2}}
$$

1. Equations of equilibrium. Form of solution for a plate free fiom bodily force.

The equations of equilibrium of a homogeneous isotropic elastic solid are of the form

$$
\begin{aligned}
& d \hat{x}+\frac{d \hat{x y}}{d y}+\frac{d \hat{z}}{d \lambda}+\mathrm{X}=0 \\
& d \widehat{x y}+\frac{d \widehat{y y}}{d y}+\frac{d \hat{y z}}{d x}+\mathrm{Y}=0 \\
& d x+d \hat{y}+d \hat{y}+z=0
\end{aligned}
$$

where $\mathrm{X}, \mathrm{Y}, \mathrm{Z}$ are the components of the bodily force per unit volume, and $\overline{x . r}, \overline{y y}, \ldots$ $\widehat{x y}, \hat{x z}, \widehat{y z}$ are the components of stress, these being given in terms of the displacements $u, v, w$ by the equations

where

$$
\begin{aligned}
& \widehat{x x}=\lambda د+2 \mu \frac{d u}{d x} \quad, \quad \bar{y}=\mu\left(\frac{d x}{d y}+\frac{d y}{d x}\right)
\end{aligned}
$$

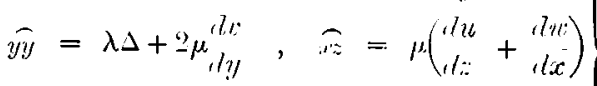

$$
\begin{aligned}
& \therefore=\lambda \Delta+2 \mu \frac{d z}{d z}, \quad \gamma_{y}=\mu\left(\frac{d n}{d x}+\begin{array}{l}
d u \\
d y
\end{array}\right)
\end{aligned}
$$

$$
\Delta=\frac{i n}{d x}+\frac{d y}{d y}+\frac{d y}{d y} .
$$


In terms of the displacements, the chuations of equilibrium are therefore

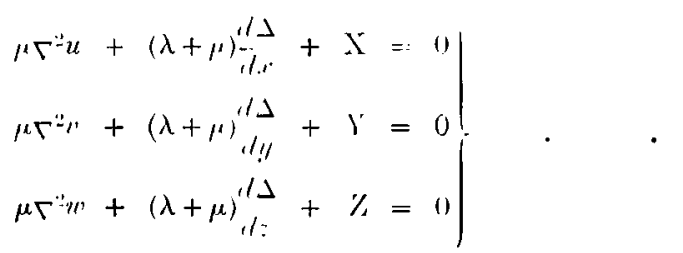

When the bodily force is null, or $\mathrm{X}=\mathrm{Y}=\%=0$, the following forms are easily shown to sitisfy equations (3),

$$
\begin{aligned}
& \text { (i) } "=2^{\prime(t)} \cdot() \\
& v=-2^{\prime \prime} \cdot 1.1 .
\end{aligned}
$$

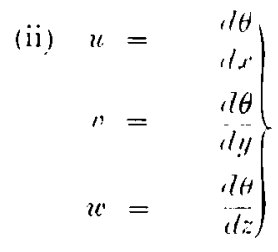

$$
\begin{aligned}
& \text { (iii) } "=\begin{array}{c}
\lambda+3 \mu d \phi \\
\lambda+\mu d l
\end{array}+2 \cdot d l^{2} \phi
\end{aligned}
$$

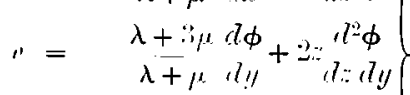

$$
\begin{aligned}
& x=-\frac{\lambda+3 \mu d \phi}{\lambda+\mu d z+2: t=\phi} d z^{2}
\end{aligned}
$$

where $\psi, \theta, \phi$ are potential functions, so that

$$
\Gamma^{\prime \prime} \psi=0, \quad \Gamma^{\prime \prime} \psi=0, \quad \Gamma^{*} \phi=0 .
$$

These solutions have been used by Bodssinese in his treatment of the problem of a solid bounded by a single plane $z=0$. They are equally effective when the boundary consists of two parallel $:$-planes. Thus, as will explicitly appear in the sequel, and as might be prover at once, any solution of (3), with $\mathrm{X}=\mathrm{Y}=\mathrm{Z}=0$, in the space between the planes $:= \pm h$, can be expressed in the form

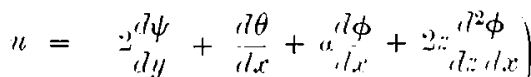

$$
\begin{aligned}
& r=-2_{d}^{d} \psi+\frac{d \theta}{d !}+a \frac{d \phi}{d y}+2 y, d z \phi
\end{aligned}
$$

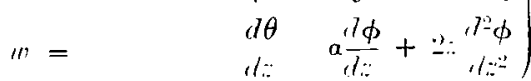

Here, and throughout the paper, the symbol a is used to lenote the fraction $(\lambda+3 \mu)$ $(\lambda+\mu)$.

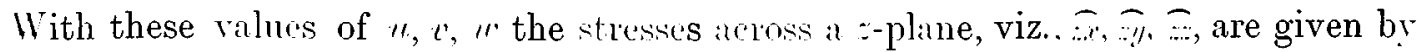

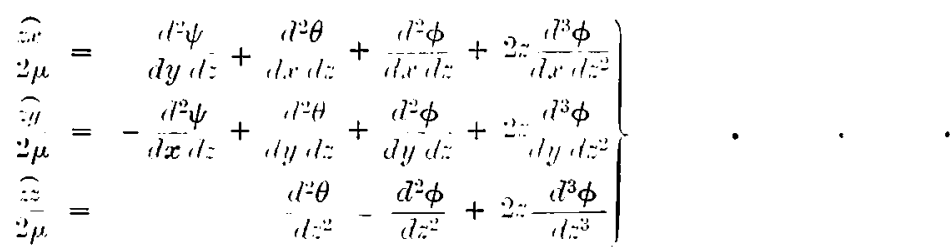


2. Foree applinel at a single point.

Returning to the solutions (i), (ii), (iii), we note that (i) and (ii) contribute nothing to the dilatation $\Delta$, and (ii), (iii) nothing to the $z$-rotation $\omega_{3}=\frac{1}{2}\left(\frac{d r}{d x}-\frac{d u}{d y}\right)$.

These properties can be used to resolve any given displacement into its $\psi, \theta, \phi$ components, the bodily force being null.

An example of fundamental importance is the displacement in an infinite solid due to a single force applied at a given point. Thus for a unit force applied at the origin in the direction of the axis of $z$ we have

$$
\left.\begin{array}{l}
u=\frac{x}{r^{3}} \\
v=\frac{y z}{r^{3}} \\
w=\frac{z^{2}}{r^{3}}+\frac{a}{r}
\end{array}\right\} \text { each multiplied l } \frac{1}{8 \pi \mu} \frac{\lambda+\mu}{\lambda+2 \mu}=\frac{1}{4 \pi \mu(a+1)}
$$

where $r^{2}=x^{2}+y^{2}+z^{2}$; or say. for a $Z$ force of $4 \pi \mu(\alpha+1)$ units applied at $\left(x^{\prime}, y^{\prime}, z^{\prime}\right)$ we have

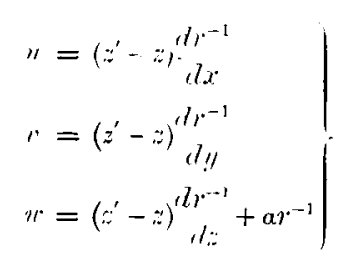

$r^{-1}$ being written for $1 / r$, where $r$ is the distance from $(x, y, z)$ to $\left(x^{\prime}, y^{\prime}, z^{\prime}\right)$.

'These give

But in (4)

$$
\Delta=(a-1) \stackrel{d r}{\prime r}: \omega_{3}=0
$$

$$
\Delta=2(1-a) \frac{d^{2} \phi}{d l z^{2}} ; \omega_{0::}=\frac{d^{2} \psi}{l z^{2}}
$$

Hence we take $\psi=0$, and choose $\phi$ so that $\frac{d \phi}{d i}=-\frac{1}{2} r^{-1}$.

Now the functions $\log \left(r+z-z^{\prime}\right)$ and $-\log \left(r-z \overline{-z^{\prime}}\right)$ are both potentials having $r^{-1}$ for $z$-derivative; the former is without singular point in the region $:>z^{\prime}$, the latter in the region $z<z^{\prime}$. We may without confusion use a single symbol to denote either function indifferently, and define

We may therefore take

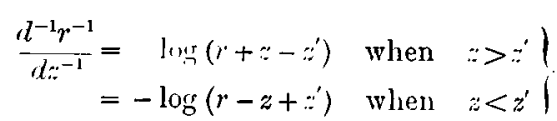

$$
\phi=-\begin{array}{cc}
1 & d l^{-1}, r^{-1} \\
\underline{2} & d v^{-1}
\end{array} .
$$

Comparison of the displacement $w$ in (4) and (i) gives now

$$
d \theta=z^{\prime \prime r}+r^{-1}+\frac{u}{2} r^{-1} \text {. }
$$


For a $Z$ force of $4 \pi \mu(1+1)$ units at $\left(x^{\prime}, y^{\prime},:^{\prime}\right)$ we have therefore

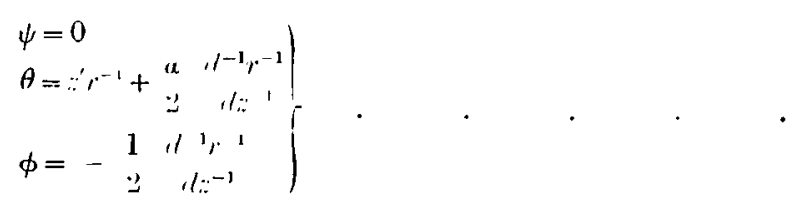

It is casy to verify that these values of $\psi, \theta, \phi$ substituted in (4) do actually reprorluce equations (6).

Similarly for an $X$ force of $4 \pi \mu(a+1)$ units at $\left(x^{\prime}, y^{\prime}, y^{\prime}\right)$ we find

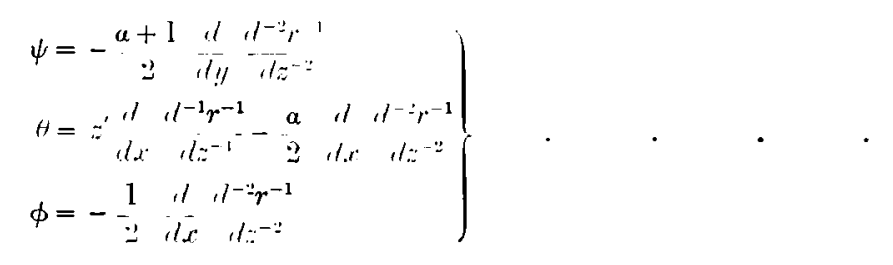

Here ${ }^{\prime l^{-*} y^{-1}}$ denotes a potential function having ${ }^{1 z^{-1} r^{-1}}$ for $i$-derivative, and is defined by the erfuations

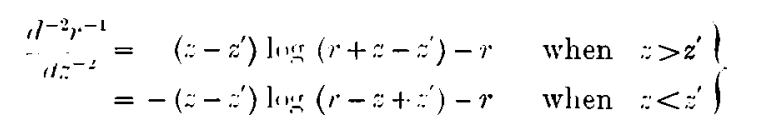

It may be observed that the necessity for dealing separately with the two regions $:>z^{\prime}$ and $z<z^{\prime}$ in these cases is not inconsistent with the theorem of (4), which refers only to a displacement free from singularity in the space considered.

\section{Solution of the problem "f normal traction.}

Coming now to the problems relating to a solid bounded by the tro parallel planes $z=h$ and $z=-h$, we begin with the simplest of these, and seek a solution of the equations of equilibrium siving

the normal stress

$$
\mathrm{X}=\mathrm{Y}=\mathrm{Z}=1 \text { throughent the burly: }
$$

$$
\begin{array}{rlrl}
-\cdots=f(x, y) & \text { on } & \because=h, \\
& =0 & \text { on } & z=-h ;
\end{array}
$$

the tangential stresses $\widehat{. .,}, \hat{z}=0$ on both faces $= \pm= \pm$. The arbitrary function $f(x, y)$, which we shall suppose to vanish at all points without a given finite area $A$, is expressed in : form amenable to analytical tritment in the familiar theorem

$$
\operatorname{Limit}_{\epsilon=0} \iint_{\left[\left(x-x^{\prime}\right)^{2}+\left(y-y^{\prime}+y^{\prime}+\epsilon^{2}\right)^{3}\right.}=2 \pi j(x, !)
$$

the integral being taken over the area $A$.

(If we imagine the plane $z=0$ to be covered with attrating matter of surface density $f(x, y)$, then the theorem expresses the well-known relation between the density at 
$(x, y, 0)$ and the limiting value of the normal attraction at $(x, y, \epsilon)$ as $\epsilon$ approaches zero.)

As a preliminary to the general problem, we take therefore the special case in which $z=$ on $z=h$ is equal to $\varepsilon /\left\{\left(x-x^{\prime}\right)^{2}+\left(y-y^{\prime}\right)^{2}+\epsilon^{2}\right\}^{1}$, or, in the form of a definite integral,

where

$$
\int_{10}^{\kappa}\left({ }^{\prime \prime}-\kappa \varepsilon_{\kappa} \times\right]_{0}(\kappa \mathrm{R}) d \kappa
$$

$$
\mathrm{R}^{2}=\left(x-x^{\prime}\right)^{2}+\left(! y-y^{\prime}\right)^{2}
$$

Making a further reduction, we begin by taking, in place of this integral, simply the function $\kappa \mathrm{J}_{0} \kappa \mathrm{R}$.

The function $\psi$ is not required, and $\phi, \theta$ are of the forms

$$
\left.\begin{array}{l}
\phi=\left(\mathrm{C}_{1} \sinh \kappa *+\mathrm{C}_{3} \cosh \kappa z\right) \mathrm{J}_{1} \times \mathrm{R} \\
\theta=\left(\mathrm{C}_{3} \sinh \kappa *+\mathrm{C}_{4} \cosh \kappa ;\right) \cdot \mathrm{J}_{1,} \mathrm{R}
\end{array}\right\}
$$

In accordance with (5) these satisfy the conditions

Hence we easily find

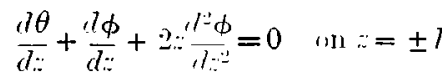

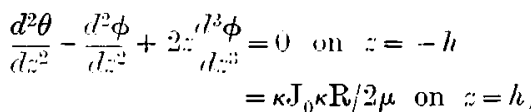

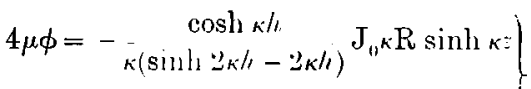

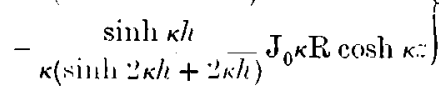

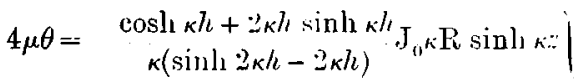

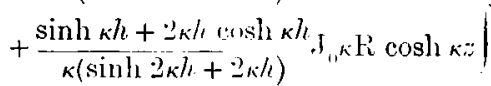

If these expressions, multiplied by $e^{-\kappa \epsilon}$, could be integrated with respect to $\kappa$ from 0 to $\infty$, we should have at once a solution of the preliminary problem. But this integration is not possible, owing to the nature of the functions of $k$ near the lower limit $\kappa=0$. In fact, if the values of $4 \mu \dot{\phi}, 4 \mu \theta$ in (13) be expanderl in ascending powers of $\kappa$, the expansions will contain terms in $1 / \kappa^{3}$ and $1 / \kappa$, so that neil $\kappa=0$

$$
\begin{aligned}
4 \mu \phi & =\mathrm{H} / \kappa^{3}+\mathrm{K} / \kappa+\text { terms of positive llogre" } \\
4 \mu \theta & =\mathrm{L} / \kappa^{3}+3 / \kappa+", "
\end{aligned}
$$

These terms of negrtive degree are potentials contributing nothing to the stresses on $z= \pm h$, as we see from $(12)$, since $\kappa_{0} \mathrm{~J}_{1} \mathrm{R}$ contains no terms of newintive degree. They might therefore he subtracted from the expressions (13) without affecting the satisfaction of the conditions in (12). This simple subtraction would, however, introduce terms not integrable right up to the upper limit, at loust after $c$ is put equal to zero, as eventually it will be. The difficulty is met by subtricting from $4 \mu \phi$, not $\mathrm{H} / \kappa^{3}+\mathrm{K} / \kappa$, but $\mathrm{H} / \kappa^{3}+\mathrm{K} e^{-\kappa h} / \kappa$; and from $1 \mu \omega$, not $\mathrm{L} / \kappa^{3}+\mathrm{II} / \kappa$, but $\mathrm{I}_{2} / \kappa^{3}+\mathrm{M} e^{--\kappa h} / \kappa$. 
(There are, of course, any number of equally suitable modifications; instead of ${ }^{-\alpha h}$ we might take $e^{-x c}$ or $1 /\left(1+\kappa^{2}\right)$, for instance.) A solution of the preliminary problem of normal traction equal to $\epsilon /\left(\mathrm{R}^{2}+\epsilon^{2}\right)^{\xi}$ on the face $z=h$ is thus obtained in the form

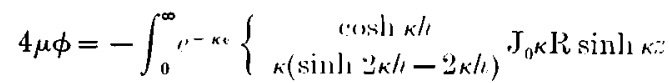

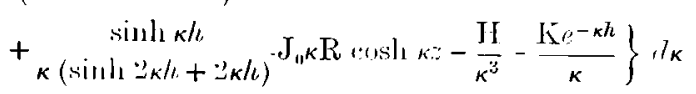

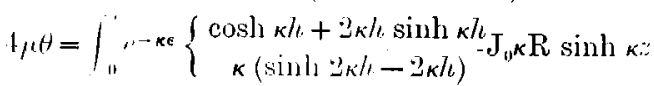

$$
\begin{aligned}
& \left.+\frac{\sinh \kappa h+2 \kappa h \cosh \kappa h \cdot}{\kappa(\sinh 2 \kappa h+2 \kappa h)} \cdot \mathrm{J}_{0} \kappa \mathrm{R} \cosh \kappa:-\frac{\mathrm{L}}{\kappa^{3}}-\frac{3\left[\rho^{-\kappa h}\right.}{\kappa}\right\} d \kappa
\end{aligned}
$$

The solution of the original general problem is found by multiplying by $f^{\prime}\left(x^{\prime}, y^{\prime}\right) / 2 \pi$, integrating with respect to $x^{\prime}, y^{\prime}$ over the area $\mathrm{A}$, and finally taking the limit for $\epsilon=0$. But a glance at the forms near $\kappa=\infty$ of the functions in (14) shows that the triple integrals are absolutely convergent, it being supposed that $-h<z<h$. Hence we may integrate with respect to $x^{\prime}, y^{\prime}$ first, and by a wellknown theorem the limits for $\epsilon=0$ may then be found by simply putting $\epsilon=0$ in the integrands, provided the resulting integrals are convergent, as they manifestly are.

This gives the value of $\phi$, for example, in the form

$$
\int_{0}^{\infty} d \kappa \iint f\left(x^{\prime}, y^{\prime}\right) \mathrm{F}(\kappa) d x^{\prime} d y^{\prime}
$$

but, always provided $-h<z<h$, we may change this if we please into

$$
\iint f\left(x^{\prime}, y^{\prime}\right) d x^{\prime} d y^{\prime} \int_{0}^{\infty} \mathrm{F}(\kappa) d \kappa
$$

Finally, we may with great advantage confine our study in the first place to what is usually spoken of as a urit element of normal traction at $\left(x^{\prime}, y^{\prime}, h\right)$. The area $\mathrm{A}$ enclosing this point is diminished, and the intensity of traction increased without limit, so that $\iint f\left(x^{\prime}, y^{\prime}\right) d x^{\prime} d y^{\prime}$ remains equal to unity. The resulting solution is simply that of (14), but with $\epsilon$ put equal to zero within the integral signs.

As we have just seen, the solution for the general case can at any time be found from this elementary solution (15) by multiplying by $f^{\prime}\left(x^{\prime}, y^{\prime}\right) / 2 \pi$ and integrating over the area $A$.

4. Flexural and extensional components of the strain. Disadvantages of the solution in clefinite integrals.

In the elementary solution each of the potentials $\phi, \theta$ may with advantage be decomposed into an odd and an even part in $z$. Thus, for an element of normal.

TRANS. ROY. SOC. EDIN., VOL. XLI. PART I. (NO. 8). 
traction of $8 \pi \mu$ units at $\left(x^{\prime}, y^{\prime}, h\right)$, a solution is given by

where

$$
\phi=\phi_{0}+\phi ; \theta=\theta_{0}+\theta_{\text {. }}
$$

$$
\begin{aligned}
& \left.\begin{array}{l}
\phi_{0}=\int_{0}^{\infty}\left\{-\frac{\cosh \kappa h}{\kappa(\sinh 2 \kappa h-2 \kappa h)} \mathrm{J}_{0^{\kappa}} \mathrm{R} \sinh \kappa z+\frac{3 z}{4 \kappa^{3} h^{3}}+e_{\kappa}^{-\kappa h}\left(\frac{2 z^{3}-3 \mathrm{R} z}{16 h^{3}}+\frac{9}{40} z\right)\right\}, l_{\kappa} \\
\theta_{11}=\int_{11}^{\kappa}\left\{\frac{\cosh \kappa h+2 \kappa h \sinh \kappa h}{\kappa(\sinh 2 \kappa h-2 \kappa h)} \mathrm{J}_{0} \kappa \mathrm{R} \sinh \kappa z-\frac{3 z}{4 \kappa^{3} h^{3}}-\frac{\rho-\kappa h}{\kappa}\left(\frac{2 z^{3}-3 \mathrm{R}^{3} z}{16 h^{3}}+\frac{9}{40} \frac{z}{h}+\frac{3}{2} \bar{h}\right)\right\} d \kappa
\end{array}\right\} \\
& \left.\phi_{\ell}=\int_{0}^{\infty}\left\{-\frac{\sinh \kappa h}{\kappa(\sinh 2 \kappa h+2 \kappa h)} \mathrm{J}_{0} \kappa \mathrm{R} \cosh \kappa z+\frac{e^{-\kappa h}}{4 \kappa}\right\} d \kappa\right) \\
& \left.\theta_{\varepsilon}=\int_{0}^{\infty}\left\{\frac{\sinh \kappa h+2 \kappa h \cosh \kappa h}{\kappa(\sinh 2 \kappa h+2 \kappa h)} J_{\| 1} \mathrm{R} \cosh \kappa z-\frac{3 e^{-\kappa h}}{4 \kappa}\right\} . \lambda_{\kappa}\right\}
\end{aligned}
$$

The conditions satisfied at the faces by the partial solutions (16) and (17) are easily made out. For when $\phi, \theta$ are both odd functions of $z$, then $\approx x$, $z$, are even and $\bar{z}$ odd; but when $\phi, \theta$ are even functions of $s$, then $\widehat{x} \bar{z}, \bar{y}$ are odd, $\bar{z} z$ even, as is obvious from (5). Hence (16) gives equal values of opposite sign for $z:$ at corresponding points on $:= \pm h ;(17)$ gives equal values of the same sign.

It follows that (16) is the solution for elements of normal force of $4 \pi \mu$ units at each of the points $\left(x^{\prime}, y^{\prime}, h\right),\left(x^{\prime}, y^{\prime},-h\right)$, the force being in the positive direction of $0 \%$ in each case, and therefore a traction on $z=h$, but a pressure on $z=-h$; in (17) the only difference is that the force is a traction on both planes.

Hence, also, (16) subtracted from (17) will give the solution for traction on $:=-h$ alone.

Each of the integrals in (16), (17) defines a potential function without singularity at a finite distance in the space between the planes $z= \pm l$, and all the successive derivatives with respect to $x, y, z$ of any of these functions may be calculated by differentiation within the sign of integration, provided we are dealing with a point actually within the solid, so that $-h<z<h$.

'The solutions defined by these integrals are therefore formally satisfactory. It is, however, a serious objection to them that they do not lend themselves readily to interpretation, and it is not easy to make out from them any of the simple laws which the ordinary approximate theory leads us to anticipate.

In particular, the solutions in their present form throw no light on the question of the behaviour of the functions and their derivatives at points the distance of which from the sources of strain is great in comparison with the thickness of the plate, a question of great importance for the application to the thin-plate theory.

The analytical transformations to which we now proceed reduce the solutions to a form entirely free from these objections. Each of the integrals is shown to be composed of two parts of very different character. The first part represents a function the value of which diminishes with great rapidity as the distance from the source increases, while the remaining part is a function of very simple form. Each solution is thus resolved into a permanent or persistent element and a local, transitory, or decaying element, the latter being insignificant beyont the immediate vicinity of the source. 


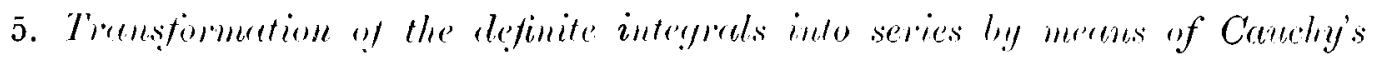

Theorem.

The integral $w_{n}$ of (16) am lee written as the sum of three integrals, armely-

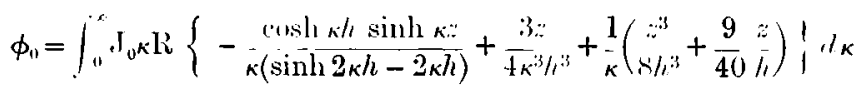

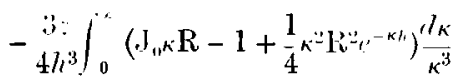

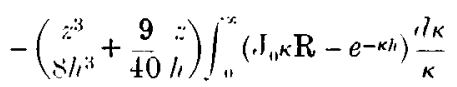

It should be olsserver that the first and third of these integrals cease to converge when $\mathrm{R}=0$. Hence the transformation does not apply to points on the line $x=x^{\prime}, y=y^{\prime}$, the normal to the plate through the sources.

Consider now the first integral in (18). The function of $\kappa$ multiplying $\mathrm{J}_{0} \kappa \mathrm{R}$ within the integral sign is an odd function of $\kappa$ vanishing for $\kappa=0$. Hence, as in $(i)$, the integral is equivalent to the complex integral

$$
\int \frac{1}{\pi i}\left(i_{0} \kappa \mathrm{R}\left\{-\frac{\cosh \kappa h \sinh \kappa z}{\kappa\left(\sinh i^{2} \alpha h-2 \kappa \lambda_{1}\right.}+\frac{3 z}{4 \kappa^{3} h^{3}}+\frac{1}{\kappa}\left(\frac{z^{3}}{8 h^{3}}+\frac{9}{40} \frac{z}{h}\right)\right\} d \kappa,\right.
$$

the path of integration running from west to east along the whole of the real axis, and just avoiding the origin, which is a singular point of $\mathrm{G}_{0} \kappa \mathrm{R}$, on the north or upper side.

On this path take points $\mathrm{E}, \mathrm{W}$ at distances $\| \pi / 2 h$ to the right and left of the origin, and on $\mathrm{EW}$ as side describe a square $\mathrm{EWAB}$ in the upper part of the plane. The integral over each of the sides $W A, A B, B E$ is easily proved to have zero for limit when $u$ tends to infinity through positive integral values.

Hence, by Cauchy's fundamental theorem, the integral over the path $\mathrm{W} E$ is equal to the sum of the residues of the integrand at its poles in the upper half of the $\kappa$ plane, multiplied by $2 \pi i$, that is, to the series

$$
\sum_{\kappa}\left(i_{0} \kappa R(-) \frac{\cosh \kappa^{h} \sinh \kappa: i}{\kappa h(\cosh 2 \kappa h-1)},\right.
$$

the summation extending over the zeroes of the function sinh $2 \kappa h-2 \kappa h$ in the upper half of the $\kappa$ plane, in the order of their moduli.

If $\zeta_{n}$ is a zero of the function $\sinh \zeta-\zeta$, the corresponding zero of $\sinh 2 \alpha h-2 \kappa h$ is $\kappa_{n}=\zeta_{n} / 2 h$, and

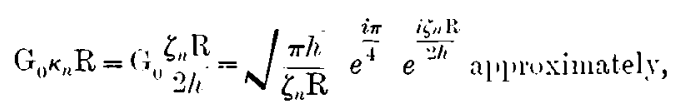

and we see that this part of $\phi_{0}$, with its successive derivatives, is practically insensible when $\mathrm{R}$ is a very moderate multiple of $2 h$. (Ct. $\S 7$, intia.) 
As to the other two integrals which occur in (18), we have proved in $(h)$ that

and

$$
\begin{aligned}
& \int_{0}^{\infty}\left(\mathrm{J}_{0} \kappa \mathrm{R}-1+\frac{1}{4} \kappa^{2} \mathrm{R}^{2} e^{-\kappa h}\right) \frac{d \kappa}{\kappa^{3}}={ }_{4}^{1} \mathrm{I}^{2} \log \frac{\mathrm{R}}{2 / \ell}-\frac{1}{4} \mathrm{R}^{2}, \\
& \int_{0}^{\infty}\left(\mathrm{J}_{0} \kappa \mathrm{R}-e^{-\kappa h}\right) \frac{d \kappa}{\kappa}=-\log \frac{\mathrm{R}}{2 h} .
\end{aligned}
$$

These functions will occur so often that it will be convenient to reserve an invariable symbol for the former of them, say

and then

$$
\left.\begin{array}{rl}
\chi(\mathrm{R}), \text { or simply } \chi, & =\frac{1}{4} \mathrm{R}^{2} \log \frac{\mathrm{R}}{2 h}-{ }_{4}^{1} \mathrm{R}^{2} \\
\Gamma^{2} \chi & =\log \frac{\mathrm{R}}{2 \lambda}
\end{array}\right\} .
$$

The persistent part of $\phi_{0}$ is therefore

$$
-\frac{3 z}{4 h^{3}} x+\left(\frac{z^{3}}{8 h^{3}}+\frac{9}{40} \frac{a}{7}\right) \nabla^{2} x,
$$

which is the sum of two potential functions,

$$
-\frac{3}{4 h^{3}}\left(z \chi-\frac{1}{6 \gamma^{3} \nabla^{2} \chi}\right) \text { and } \frac{9}{40 h^{*} \nabla^{2} \chi}
$$

6. Types of the particular solutions composing the general solution.

A glance at the relation between the results just obtained and the form of $\phi_{0}$ in (16) enables us to write down at once the corresponding transformations of $\theta_{0}, \phi_{e}, \theta_{e}$.

Collecting the results, we find

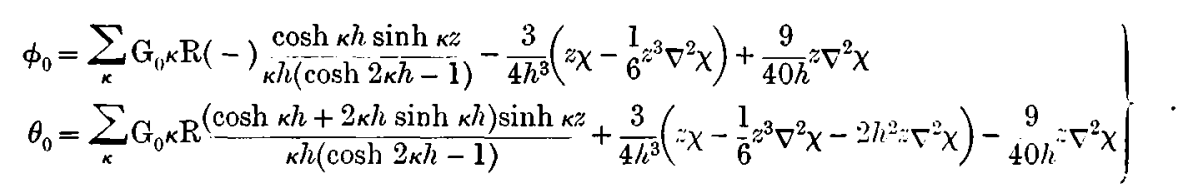

where $\kappa$ is a zero of $\sinh 2 \kappa h-2 \kappa h$, with positive imaginary part.

$$
\left.\begin{array}{l}
\phi_{e}=\sum_{\kappa}\left(i_{0} \kappa \mathrm{R}(-) \frac{\sinh \kappa h \cosh \kappa: z}{\kappa h h(\cosh 2 \kappa h+1)}+\frac{1}{4} \Gamma^{2} \chi\right. \\
\theta_{e}=\sum_{\kappa} G_{t} \kappa \mathrm{R} \frac{(\sinh \kappa h+2 \kappa h \cosh \kappa h) \cosh \kappa z}{\kappa h(\cosh 2 \kappa h+1)}-3_{4}^{3} \Gamma^{2} \chi
\end{array}\right\}
$$

where $\kappa$ is a zero of $\sinh 2 \kappa h+2 \kappa h$, with positive imaginary part.

The solution must give $\widehat{x}, \widehat{z y}, \widehat{z i}$ all equal to zero at the two plane faces of the plate, except when $R=0$, and we are thus prepared to find that the strain defined by the terms corresponding to any one root $\kappa$ gives zero stress across $z= \pm h$. Thus in (20) $\sum_{k}$ contains a series of particular solutions of the type

$$
\left.\left.\begin{array}{l}
\phi=\quad-\cosh \kappa h \sinh \kappa: \mathrm{F}(x, y) \\
\theta=(\cosh \kappa l+2 \kappa h \sinh \kappa h) \sinh \kappa: \mathrm{F}(x, y)
\end{array}\right\} \begin{array}{r}
\text { where }\left(\nabla^{2}+\kappa^{2}\right) \mathrm{F}=0 \\
\sinh 2 \kappa h-2 \kappa h=0
\end{array}\right\} .
$$


Caleulating the stresses by means of (5), we find

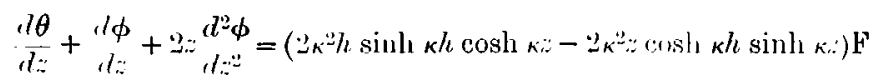

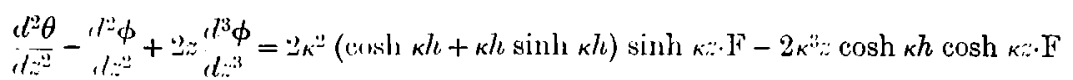

both of which vimish when $:= \pm 1$.

We have further in $(20)$ a solution of the type

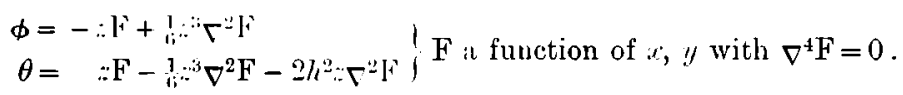

From this, by $(4),(5)$,

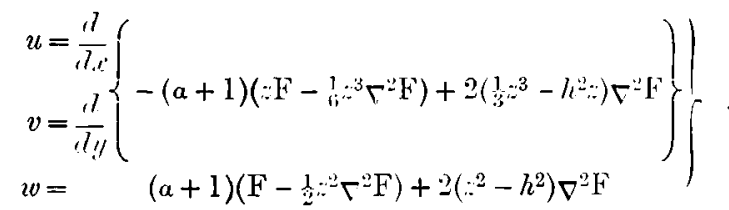

$$
\begin{aligned}
& \widehat{\omega r}=4 \mu\left(\because^{2}-h^{2}\right) \frac{d}{d x} \nabla^{2} \mathrm{~F} \\
& \left.\begin{array}{l}
\hat{z ! \prime}=4 \mu\left(\varkappa^{2}-7 \eta^{2}\right) \frac{d}{d !} \nabla^{\prime \prime} \mathrm{F} \\
\approx=0
\end{array}\right\}
\end{aligned}
$$

The solution for unit normal traction on $z=h$ contains a strain of this type, with $\mathrm{F}=\chi(\mathrm{R}) \cdot 3 / 32 \pi \mu h^{3}$.

Lastly, in (20) there is a solution of the type

giving

$$
\left.\begin{array}{c}
\phi=-: \mathrm{F} \\
\theta=\mathrm{F}
\end{array}\right\} \begin{aligned}
& \mathrm{F} \text { a function of } x, ! \\
& \text { with } \nabla^{2} \mathrm{~F}=0,
\end{aligned}
$$

$$
\begin{aligned}
& u=-(a+1) \frac{l \mathrm{~F}}{\eta, r} \\
& u=-(a+1) ! \mathrm{F} \\
& \eta=(a+1) \mathrm{F}
\end{aligned}
$$

Obviously this is merely a degenerate case of (ii).

Again in (21) we have a series of solutions of the type

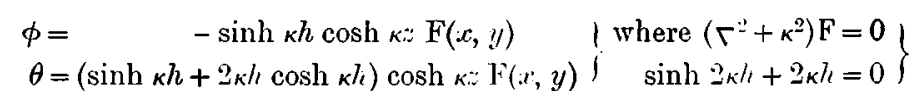

In this, as in (i) $\cong=\because=\because=0$ on $:= \pm h$.

(v) The persistent part of $(* 1)$ is of the type

giving

$$
\left.\begin{array}{l}
\phi=\mathrm{F}(x, y) ; \theta=-3 \mathrm{~F}(x, !), \text { where } \Gamma^{2} \mathrm{~F}=0 \\
"=(a-3) \frac{d \mathrm{~F}}{d !}, r=(a-3) \frac{d \mathrm{~F}}{d y} ; \widehat{r}=\widehat{z ! y}=\widehat{z}=0
\end{array}\right\}
$$




\section{Position of the zeroes of the functions $\sinh \zeta \pm \zeta$.}

The nature of the infinite series occurring in the above. solution will be made more intelligible by a short discussion of the position of the roots of the functions $\sinh 2 \kappa h \pm 2 \kappa h$. These are obviously found from the corresponding roots of $\sinh \zeta \pm \zeta$ by dividing by $2 h$.

(i) $\sinh \zeta-\zeta=0$.

$\zeta=0$ is a triple root, and the remaining roots are all complex, falling into sets of 4 of the form $\pm p \pm i q$, where $p, q$ are real. If, then, $\zeta=\xi+i \eta$, $\xi$ and $\eta$ being real, we need only consider the case of $\xi$ and $\eta$ both positive. We have then

$$
\sinh \xi \cos \eta=\xi \text { and } \cosh \xi \sin \eta=\eta \text {. }
$$

$\operatorname{Cos} \eta$ and $\sin \eta$ are therefore both positive, and $\eta$ must lie between $2 n \pi$ and $2 n \pi+\pi / 2$. It is easy to prove that there is no root between 0 and $\pi / 2$. For $\xi>\tanh \xi$, or $\xi / \sinh \xi>1 / \cosh \xi$, so that

$$
\cos \eta>\sin \eta / \eta \text { or } \eta>\tan \eta \text { or } \eta>\pi / 2 \text {. }
$$

For every positive integral value of $n$, however, beginning with $n=1$, there is one root, and one root only, with $\eta$ between $2 n \pi$ and $2 n \pi+\pi / 2$. This will be readily seen on roughly tracing the graphs $\sinh \xi \cos \eta=\xi$ and $\cosh \xi \sin \eta=\eta$, or it may be proved by an elementary application of the Theory of Functions. Thus, if we make the variable $\zeta$ describe the contour of the rectangle formed by the four lines

$$
\eta=2 n \pi, \quad \xi=\mathrm{N}, \quad \eta=2 n \pi+\pi / 2, \quad \xi=0,
$$

where $\mathrm{N}$ is a large positive number, it will be found that the function $\nu=\sinh \zeta-\zeta$ describes once a contour enclosing the point $\nu=0$ in the $\nu$ plane. There is therefore just one point within the rectangle at which $\nu$ becomes zero.

For the large roots $\cos \eta$ must be small, or

Hence

$$
\eta=2 n \pi+\pi / 2-\epsilon \text {, where } \epsilon \text { is small. }
$$

Then

$$
\text { or } \xi=\log \left(\frac{\cosh (n+\eta=2 n \pi+\pi / 2,}{4 n+1} \pi\right) \text { approximately. }
$$

$$
\epsilon=\xi / \sinh \xi=2 \log (\overline{4 n+1} \pi) /(\overline{4 n}+1 \pi) .
$$

By successive approximation we may now find the roots as nearly as we wish, but exact values are not at all necessary, the first approximation being quite sufficient for our purpose,

(ii) $\sinh \zeta+\zeta=0$.

$$
\zeta_{n}=\log (4 n+1 \pi)+\left(2 n+\frac{1}{2}\right) \pi i \quad . \quad . \quad .
$$

In this case $\zeta=0$ is a simple root, and the rest of the roots are complex. If $\zeta=\xi+i \eta$ we have

$$
\sinh \xi \cos \eta+\xi=0 \text { and } \cosh \xi \sin \eta+\eta=0 .
$$

$\operatorname{Cos} \eta, \sin \eta$ are both negative when $\xi$ and $\eta$ are positive; hence $\eta$ lies between $(2 n-1) \pi$ 
and $(2 n-1) \pi+\pi / 2$, and it may le shown, as in the previous case, that there is actually one root with $\eta$ between these limits for all positive integral values of $n$.

Also

To a first approximation

$$
\begin{gathered}
\eta=(\cdot n-1) \pi+\pi / 2-\epsilon ; \cosh \xi=2 n \pi-\pi / 2 \\
\epsilon=2 \ln n(\overline{4 n-1} \pi) /(4 n-1 \pi) . \\
\zeta_{n}=\log (\overline{4 n-1} \pi)+\left(2 n-\frac{1}{2}\right) \pi i
\end{gathered}
$$

In addition to the roots of (27), (28) we have of course a corresponding series in the second quadrant, the images of these in the axis of imaginaries.

8. Alpmerimete fin'ms of the' $\mathrm{n}^{\text {th }}$ te'ms of the infinite series, when $\mathrm{n}$ is large.

It may be useful to give in terms of $n$ ipproximate forms for the general terms of $(20),(21)$ corresponding to the "th ${ }^{\text {th }}$ roots in the first "fuadrant.

(i) $\phi_{0}$ and $\theta_{0}$.

$$
\begin{aligned}
& \kappa h=\frac{1}{2} \log 4 n \pi+\left(n+\frac{1}{4}\right) \pi i \\
& \sinh \kappa h=\frac{1}{2} e^{\kappa h}=l_{2}(4 n \pi)^{\frac{1}{2}(g)}(n+1) \pi i \\
& \frac{\cosh \kappa h}{\kappa h(\cosh 2 \kappa h-1)}=\frac{1}{\sinh \kappa h \cdot 2 \sinh ^{2} \kappa / t}=\frac{4}{(4 \imath \imath))^{?}} e^{-(n+i) \pi i}(-i) \\
& \sinh \kappa:=\frac{1}{2}\left(e^{\alpha h}\right)_{h}^{z}-\frac{1}{2}\left(e^{\kappa h}\right)^{-}{ }_{h}^{z} \\
& =\left\{\left\{(4 n \pi)^{\frac{z}{2 h} e^{(n+1)} \frac{\pi i z}{h}}-(4 n \pi)^{-\frac{z}{2 h}} e^{-(n+1) \frac{\pi i}{h}}\right\}\right.
\end{aligned}
$$

Hence in $\phi_{0}$ the general term

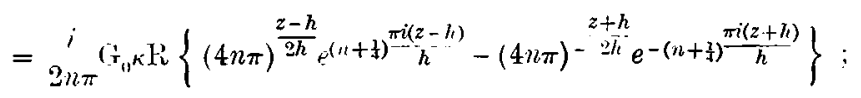

in $\theta_{0}$, the same as this, with the factor $i / 2 n \pi$ omitted.

In both

(ii) $\phi_{e}$ and $\theta_{e}$.

$$
\mathrm{C}_{\mathrm{n}, k} \mathrm{R}=\sqrt{\frac{\pi}{2 n \mathrm{R}}} \mathrm{p}^{-(n+3) \frac{\pi \mathrm{R}}{h}} e^{\frac{i \mathrm{R} \log 4 n \pi}{2 h}}
$$

$$
\begin{aligned}
\kappa h & =\frac{1}{2} \log 4 n \pi+\left(n-\frac{1}{4}\right) \pi i \\
\cosh \kappa h & =\frac{1}{2}(4 n \pi)^{\frac{1}{2}} e^{(n-1) \pi i}
\end{aligned}
$$

In $\phi_{e}$ the general term

$$
\underset{2 n \pi}{i}\left(\mathrm{~s}_{0} \times \mathrm{R}\left\{(4 n \pi)^{\frac{z-h}{2 h}} e^{(n-1))^{\pi i(z-h)}}+(4 n \pi)^{-\frac{z+h}{2 h}} e^{-(n-1) \frac{\pi(z+h)}{h}}\right\}\right.
$$

In $\theta_{0}$ the same, with the factor $i / 2 n \pi$ omitted.

$$
\mathrm{G}_{0} \kappa \mathrm{R}=\sqrt{\frac{h}{2 n \mathrm{R}}} e^{-\left(n-\frac{1}{2}\right) \frac{\pi \mathrm{R}}{h} e^{i \mathrm{R} \log 4 \lambda \mu \pi}} .
$$

9. The solution for arlitiry normal traction. Questions for discussion.

The solution of the general problem of given normal traction requires the multiplication of the functions in $(\because 0),(21)$ by $f\left(x^{\prime}, y^{\prime}\right)$ and integration with respect to $x^{\prime}, y^{\prime}$ over a finite area $A$. There is no difficulty in showing that these integrations can be -performed term by term, and that the resulting series converge absolutely. 
When the solution of the general problem has thus been obtained in terms of series of surface integrals, several questions present themselves for treatment, among which may be specially mentioned

(i) For how many times in succession may these series be differentiated term by term with respect to the coordinates $x, y, z$ ?

(ii) When the thickness of the plate is infinitesimal, but $f(x, y)$ does not vary as $h$ tends to zero, what are the orders of the various parts of the solution, and of the related physical quantities?

(iii) How are the answers to these two questions affected by discontinuity in the applied traction, or its $x, y$ derivatives?

A perfectly general discussion of these questions would be tedious and difficult, and it will probably be more useful to consider the points suggested in the light of a special case, in which the integrations required can be performed, and the outstanding features of the solution can be grasped with comparative ease.

10. Detailed solution of a special case. Term by term differentiations.

The solution we propose to work out is to satisfy the following boundary conditions :-

$$
\begin{aligned}
& \left.\begin{array}{rl}
\hat{z}:= & 4 \pi \mu \mathrm{J}_{m}(\beta \rho) \cos m \omega, \text { on } z=h \\
& =-4 \pi \mu \mathrm{J}_{m}(\beta \rho) \cos m \omega, \text { on } z=-h
\end{array}\right\} \text { when } \rho<a \\
& =0 \quad \text { on } z= \pm h \text {, when } \rho>a \\
& \widehat{z x}=\widehat{z y}=0, \quad \text { on } z \pm h \text {. }
\end{aligned}
$$

$\rho, \omega, z$ are the cylindrical coordinates of the point $(x, y, z)$, so that $x=\rho \cos \omega$, $y=\rho \sin \omega$.

$\beta$ is any constant, and $m$ is an integer.

The solution, obtained from (20) by integration, is

$$
\begin{aligned}
& \phi=\phi_{1}+\phi_{2} ; \quad \theta=\theta_{1}+\theta_{2} \text {, where } \\
& \phi_{1}=-\frac{3}{4 h}\left(z \mathrm{~F}-\frac{1}{6} z^{3} \nabla^{2} \mathrm{~F}\right)+\frac{9}{407^{3}} \nabla^{23} \mathrm{~F} \\
& \theta_{1}=\frac{3}{4 h^{3}}\left(\because \mathrm{F}-\frac{1}{6} z^{3} \nabla^{2} \mathrm{~F}-2 / i^{*} \because \nabla^{*} \mathrm{~F}\right)-{ }_{40 h^{2}}^{9} \Gamma^{2} \mathrm{~F} \\
& \text { with } \quad F=\iint \chi(R) \cdot J_{m}\left(\beta \rho^{\prime}\right) \cos m \omega^{\prime} \rho^{\prime} d \rho^{\prime} d \omega^{\prime} \\
& \left.\phi_{2}=\sum_{\kappa}(-) \frac{\cosh \kappa h \sinh \kappa z}{\kappa h(\cosh 2 \kappa h-1)} \iint G_{0} \kappa \mathrm{RJ}_{m} \beta \rho^{\prime} \cos m \omega^{\prime} \rho d \rho^{\prime} d l \omega^{\prime}\right) \\
& \theta_{2}=\sum_{\kappa} \frac{(\cosh \kappa h+2 \kappa h \sinh \kappa h) \sinh \kappa:}{\kappa h(\cosh 2 \kappa h \quad 1)} \quad \text { (same intergral)) }
\end{aligned}
$$

the integrals being taken over the circle of radius $\because$.

Consider in the first place the part of the solution lefined by $\phi_{2}, \theta_{\mathrm{s}}$.

The value of the surface integral in (30) takes different forms when $\rho\rangle$ and $\langle a$. 
Is proved in (c)

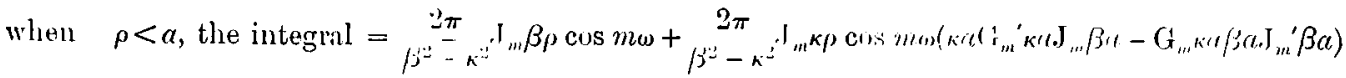

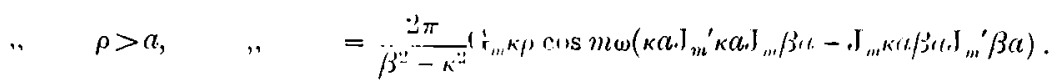

Now when $x \rho$, se : ate both large,

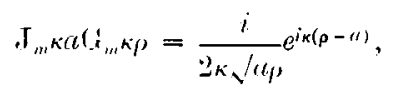

which, with its derivatives, is very small when $(\rho-\alpha) / 2 \%$ is even morlerately large. Thus. in the space without the cylinder $\rho="$, the part of the strain given by $\phi_{\dot{z}}, \theta_{2}$ is, when $h$ is small, insensible except in the immediate neighbourhood of that cylinder. The same remark applies to the strain within the cylinder, so far as it is givoul by the parts of $\phi_{2}, \theta_{2}$ arising from the second term in the value of the surface integral.

We naturally inquire, how do these rapidly decaying parts of the solution behave, and what is the order of magnitude of the corresponding displacements and stresses, at points actually on the surface $\rho=a$ ? Now, taking for example the value of $\phi_{2}$ in the external region, namely,

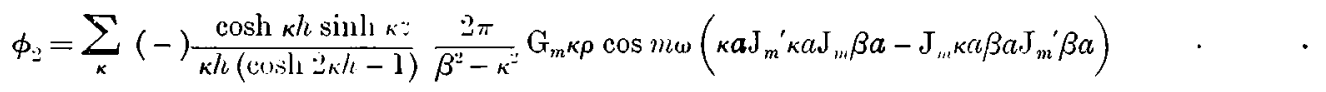

we see from $\S 8$ (i) that when $\rho=a$, the general term has the approximate form

$$
\frac{\Lambda}{n^{3}}\left\{n^{\frac{z-h}{2 h}} e^{(n+\xi) \frac{\pi i(z-h)}{h}}-n^{-\frac{z+h}{2 h h}} e^{-(n+1) \frac{\pi(z+h)}{h}}\right\}
$$

A being independent of $\mu$. Moreover, each differentiation of $\phi$ with respect to $\rho$ or $:$ will remove a factor $1 / n$ from the general term. Hence thee such differentiations, but no more, are permissible, if $-h<:<h$. But from ( $t)$ it is clear that none of the displacements requires more than two, and none of the stresses more than three of these differentiations for their calculation. As for $\theta_{2}$, the general term is of one orler higher in $n$ than the corresponding term in $\phi_{2}$, but in compensation for this only two differentiations are required to find the stresses. Hence, so far as the lecaying part of the solution is concerned, displacements and stresses at $\rho=\alpha$ may be found by means of term hy term differentiation, and sulseequent substitution of $a$ for $\rho$.

Again, considering the order of these various ifuantities in $h$, regarded as infinitesimal, and remembering that $\kappa h$ and $\kappa=$ are of onlur zero in $h$, we see thit the expression for $\phi_{2}$ in $\left(30^{\prime}\right)$ and the corresponding expression for $\theta_{2}$ are of order $h^{2}$ when $\rho=a$, and each differentiation with respect to $\rho$ or $:$ diminishes the order by one. Hence the displacements at $\rho="$ are of order $h$ ancl the stresses of order zeró, so far as they arise from the decaying part of the solution $\psi_{2}, \theta_{2}$. 
11. The same speciul problem. Summation of two infinite series.

An important part of the strain given by $\phi_{2}, \theta_{c_{2}}$ remains to be considered, namely, that which arises from the term $2 \pi /\left(\beta^{2}-\kappa^{2}\right) . \quad J_{m} \beta \rho \cos m \omega$ in the value of the surface integral for the case whon the point $(\rho, \omega)$ is within the cylinder $\rho=\alpha$. Denoting these parts of $\phi_{2}, \theta_{22}$ by $\phi_{3}, \theta_{3}$, we have

$$
\begin{aligned}
& \phi_{3}=2 \pi \mathrm{J}_{m} \beta \rho \cos m(n) \sum_{\kappa}(-) \frac{\cosh \kappa h \sinh \kappa z}{\left(\beta^{2}-\kappa^{2}\right) \kappa h(\cosh 2 \kappa h-1)} \mid \\
& \left.\theta_{3}=2 \pi J_{m} \beta \rho \cos \| \omega \sum_{\kappa} \frac{(\cosh \kappa h+2 \kappa h \sinh \kappa h) \sinh \kappa}{\left(\beta^{2}-\kappa^{2}\right) \kappa h(\cosh 2 \kappa h-1)}\right)
\end{aligned}
$$

We note in the first place that $\phi_{3}$ admits of three, and $\theta_{3}$ of two term by term differentiations with respect to $z$ when $-h<i<h$, while $x, y$ differentiations can be performed without restriction. Combining this result with those already obtained, we see that in the complete solution in terms of surface integrals, all the differentiations necessary to give the displacements and strains or stresses at any point in the body of the plate can be performed on the series term by term.

When $h$ is small, $\phi_{3}$ and $\theta_{3}$ are of order $h^{2}$, and a $z$-differentiation lowers the order by one. This can be seen from the series, or otherwise, for, as we shall now show, the value of the series can be found in finite terms.

Consider the function of $\kappa$,

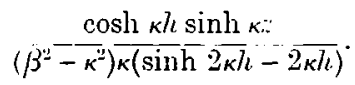

This function, multiplied by $\kappa$, vanishes at infinity at all points of the path $\mathrm{EW} \mathrm{A} \mathrm{B} \mathrm{described} \mathrm{in} \S 5$; hence the sum of its residues vanishes. The function being odd in $\kappa$, the residues at the poles $\kappa= \pm \kappa^{\prime}$ are equal. Thus

$$
\begin{aligned}
& 2 \text { (series of residues at zeroes of } \sinh 2 \kappa h-2 \kappa h \text { in upper part of plane) } \\
+ & 2 \text { (residue at } \kappa=\beta)+(\text { residue at } \kappa=0)=0 .
\end{aligned}
$$

The residue at $\kappa=\beta$ is

Also if

$$
(-) \frac{\cosh \beta h \sinh \beta z}{2 \beta^{2}(\sinh 2 \beta h-2 \beta h)} \text {. }
$$

$$
\frac{\cosh \kappa h \sinh \kappa \%}{\kappa(\sinh 2 \kappa / \iota-2 \kappa h)}=\frac{1}{\kappa^{3}}+{ }_{\kappa}^{\mathrm{B}}+\cdots, \text {, near } \kappa=0 \text {, }
$$

then the residue at $\kappa=0$ is

Hence

$$
\frac{A}{\beta^{4}}+\frac{1}{\beta^{2}}
$$

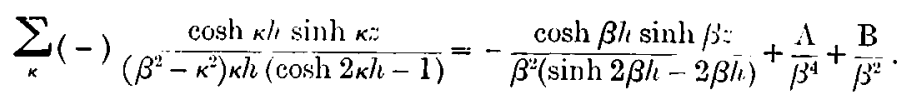

It may be noted that $\mathrm{A} / \beta^{4}+\mathrm{B} / \beta^{*}$ are simply the terms of negative degree in the expansion of

in ascending powers of $\beta$.

$$
\frac{\cosh \beta h \sinh \beta z}{\beta^{2}(\sinh 2 \beta h-2 \beta h)}
$$


llence, putting in the values of $\mathrm{A}$ and $\mathrm{B}$ from (16), (18),

$$
\begin{aligned}
& \phi_{\beta}=2 \pi J_{m} \beta \rho \cos m \omega\left\{-\frac{\cosh \beta h \sinh \beta z}{\beta^{2}(\sinh 2 \beta h-2 \beta h)}+\frac{3 z}{4 \beta^{4} h^{3}}+\frac{1}{\beta^{2}}\left(\frac{z^{3}}{8 h^{3}}+\frac{9}{40} \frac{z}{h}\right)\right\} \\
& \left.\theta_{3}=2 \pi \mathrm{J}_{m} \beta \rho \cos m \omega\left\{\frac{(\cosh \beta h+2 \beta h \sinh \beta h) \sinh \beta z}{\beta^{2}(\sinh 2 \beta h-2 \beta h)}-\frac{3 z}{4 \beta^{4} h^{3}}-\frac{1}{\beta^{2}}\left(\frac{z^{3}}{8 h^{3}}+\frac{69}{40} \frac{z}{h}\right)\right\}\right\}
\end{aligned}
$$

and similarly

12. The seme problem. Final form of the solution.

We come lastly to $\phi_{1}, \theta_{1}$ of $\S 10$.

The function $\mathrm{F}$ requires separate formulæ for its expression in the three cases $m=0, m=1, m>1$, but in all cases

$$
\begin{aligned}
& \left.\mathrm{F}=\left(2 \pi / \beta^{4}\right) \mathrm{J}_{m} \beta \rho \cos m \omega+\mathrm{F}_{1}, \quad \text { when } \rho<a\right\}
\end{aligned}
$$

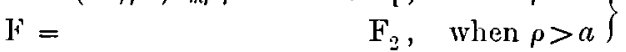

where $\mathrm{F}_{1}$ and $\mathrm{F}_{2}$ satisfy the equations $\nabla^{4} \mathrm{~F}_{1}=0, \nabla^{4} \mathrm{~F}_{2}=0$. The values of $\mathrm{F}_{1}, \mathrm{~F}_{2}$ for the various cases are given in $(f)$.

When $\rho<a$, the term $\left(2 \pi / \beta^{4}\right) J_{m} \beta_{\rho} \cos m \omega$ of $\mathrm{F}$, taken by itself in $\phi_{1}$ and $\theta_{1}$, would give

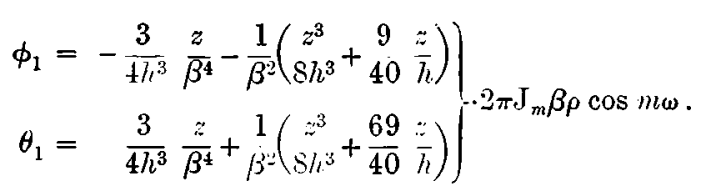

'These are precisely the terms of negative degree (both in $\beta$ and in $h$ ) with signs changed, in the expressions for $\phi_{3}$ and $\theta_{3}$ given at the end of $\S 11$.

If, then, we take this part of $\phi_{1}, \theta_{1}$ along with $\phi_{3}, \theta_{3}$ we have the complete solution in the form

$$
\begin{aligned}
& \phi=-2 \pi J_{m} \beta \rho \cos m \omega \frac{\cosh \beta h \sinh \beta:}{\beta^{2}(\sinh 2 \beta h-2 \beta h)} \\
& +2 \pi \sum_{\kappa}(-) \frac{\cosh \kappa h \sinh \kappa z}{\left(\beta^{2}-\kappa^{2}\right) \kappa /(\cosh 2 \kappa h-1)} \mathrm{J}_{m \kappa \rho} \cos m \omega\left(\kappa a \mathrm{G}_{m}{ }^{\prime} \kappa a \mathrm{~J}_{m} \beta a-\mathrm{G}_{m} \kappa a \beta a \mathrm{~J}_{m}{ }^{\prime} \beta a\right) \\
& -\frac{3}{4 h^{3}}\left(z \mathrm{~F}_{1}-\frac{1}{6} z^{3} \nabla^{2} \mathrm{~F}_{1}\right)+\underset{40 h^{9}}{9} \nabla^{2} \mathrm{~F}_{1} \\
& \theta=2 \pi \mathrm{J}_{m} \beta \rho \cos m \omega^{\left(\cos h_{2} \beta h+2 \beta h\right.} \frac{\sinh \beta h) \sinh \beta z}{\beta^{2}(\sinh 2 \cdot \bar{\beta} h-\overline{2} \beta h)}
\end{aligned}
$$

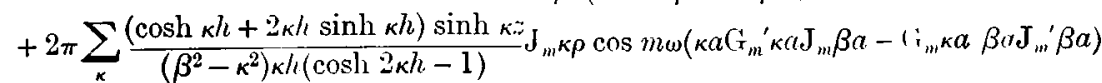

$$
\begin{aligned}
& +\frac{3}{4 h^{3}}\left(z \mathrm{~F}_{1}-\frac{1}{6} z^{3} \nabla^{2} \mathrm{~F}_{1}-2 h^{2}-\nabla^{2} \mathrm{~F}_{1}\right)-\frac{9}{40 h} \nabla^{2} \mathrm{~F}_{1} \\
& \text { when } \rho<a \text {. }
\end{aligned}
$$

and

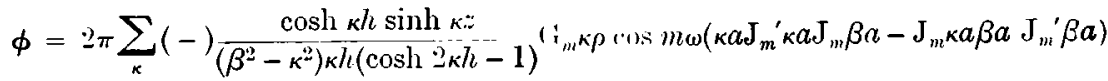

$$
\begin{aligned}
& -\frac{3}{4 h^{3}}\left(\approx \mathrm{F}_{3}-\frac{1}{6}-\nabla^{2} \mathrm{~F}_{2}\right)+\underset{+40 / i^{2}}{9}-\nabla^{2} \mathrm{~F}_{2}
\end{aligned}
$$

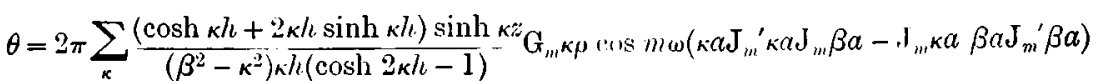

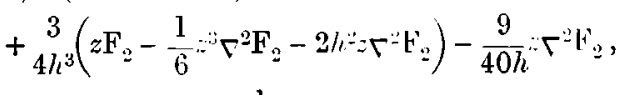

$$
\begin{aligned}
& \text { when } \rho>a \text {. }
\end{aligned}
$$


ln (33) and (31) each line represents a potential function; in (33) the first lines define a particular solution giving the proper values of the tractions at the surface, as may be seen from (13); the partial solutions given by the $\kappa$ series give zero surface tractions, and represent a strain insensible except in the vicinity of $\rho=\alpha$; and the solutions defined by the last lines, being of the form (23), give zero surface tractions.

From these remarks it follows immediately that the solution (33), (34) satisfies all the conditions of the problem in the two regions $\rho\langle\alpha, \rho\rangle \alpha$, taken separately. To verify the solution completely, it would be necessary to show in addition that certain conditions are fulfilled at the surface $\rho=a$, namely,

(i) that the displacements and strains are continuous at this surface, and

(ii) that the integral value of the stresses $\ldots, \ldots$ over any small area lying partly within and partly without the cylinder $\rho=a$, on either of the plane faces of the plate, tends to zero when the area is indefinitely diminished.

The condition (i) ensures the 'synexis' of the solution across the surface $\boldsymbol{\rho}=a$, and can be proved by showing, as may easily be done by means of summations similar to those of $\S 11$, that $\phi, \theta, \frac{d \phi}{d \rho}$ and $\frac{d \theta}{d \rho}$ are continuous at that surface. For by the Theorv of the Potential this carries with it the continuity of all the derivatives of $\phi$ and $\theta$, and therefore of the displacements and stresses, as well as of all their derivatives, uncler the proviso, of course, that $-h<z<h$.

The condition (ii), or some equivalent, is required in order to exclude the possibility of stresses with finite resultant passing into the solid through the lines $z= \pm h, \rho=\imath$; or, in other words, in order to ensure that the solution is not partly due to linear elements of traction at these lines.

\section{1.) Order of the varions parts of the solution, when $\mathrm{h}$ is small.}

The final form of the solution, as exhibited in (33), (34) was obtained by combining parts of $\phi_{1}, \theta_{1}$ with $\phi_{2}, \theta_{2}$, and until this was done, it was not immediately evident that $\phi$ and $\theta$ were potentials. Thus the part of the solution arising from the imaninary values of $\kappa$, or from any one of them, is not, within the region of applied traction, a potential by itself, and the same is true of the $\phi_{1}, \theta_{1}$ part, which may be considered as coming from the zero values of $\kappa$. This has sometimes to be taken into account in calculating the stresses; the formula for $\widehat{m}$, for eximple, in (i) rey uires arlitional terms if, while $u, v, w$ are still given by $(t), \phi$ and $\theta$ are not potentials.

On the other hand, the separation of the solution into the two palls (29), (30) has this very marked advantage that, when $h$ is very small, the first part gives the terms of the two lowest orders in $h$ of $\psi, \theta, 1$ inmely those of orless $h^{-*}$ and $h^{0}$, while the second part, as we have already seen, contiains no terms of lower orler than $1 i^{2}$. When, however, we come to calculate displacements and stresses, the separatimn is less simple, mainly in consispuence of the fact that $x^{\prime}, y$ differentiations clo not change the order of $\phi_{1}, \theta_{1}$, but diminish the order of $\psi_{2}, \theta_{2}$ by one for each differentiation. 
The following table, which may $\mathrm{l}$ c deduced immediately from the results of $\$ \$ 10,11$, show's the order in hef displacements and stresses arising from $\phi_{1}, \theta_{1}$ and $\phi_{2 .}, \theta_{2}$ respectively.

\begin{tabular}{|c|c|c|c|}
\hline & & & \\
\hline & $\phi_{1}, \theta_{1}$ & 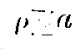 & $p=a$ \\
\hline$u, v$ & $-2,0$ & $y$ & 1 \\
\hline wo & $-3,-1$ & 1 & 1 \\
\hline$\widehat{x} \cdot \hat{x}, \widehat{x y}, \widehat{y y}$ & $-2,0$ & 0 & 0 \\
\hline 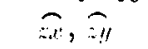 & -1 & 1 & 0 \\
\hline$\approx$ & 0 & 0 & 0 \\
\hline
\end{tabular}

It thus ippeils that the first part of the solution fives all the displacements to a second approximation, and all the stresses but $-:-$ to a first approximation. With regard to $\approx$, it should be observed that the solutions depenling on $\mathrm{F}_{1}, \mathrm{~F}_{2}$ contribute nothing to it, so that, within $\rho=\alpha$, its value comes altogether from the particular solution, and without $\rho=a$, its value is zero beyond the immediate vicinity of that surface.

14. Methods and results of the special case extended to the general problem of arbitrary normal traction.

One feature of the solution expressed by equations (33), (34) we have already found useful, especially in the important case when $h$ is small, in such a way that $\beta h$ and $l / a$ are small fractions. We refer to the explicit spentration in the solution of a purely local elemcut, entirely negligible except, within a certain strip of breadth comparable with the thickness of the plate, from an element of a persistent or permanent character, with an area of influence not affected by the inclefinite diminution of $h$.

Another advantage of the form of solution in (38), (34) is that the particular solution for the space within which the traction is applied is found in such a form that it can be readily expanded in powers of $h$, so as to wive the terms of positive order in the infinitesimal $h$, as well as those of negative order which were already separated in (29). Thus in the particular solution, or first line of $\phi$ in (33), the factor

$$
\frac{\cosh \beta h \sinh \beta:}{\beta^{2}(\sinh 2 \beta h-2 \beta h)}
$$

can be expanded in ascending powers of $\beta$, the series converging if $|\Xi B h|<\left|\zeta_{1}\right|$, where $\zeta_{1}$ is the complex root of $\sinh \zeta-\xi=0$ with smallest morlulus. Since : is of the same order as $h$, and we are supposing $\beta$ independent of $h$, it is clear that the terms of the series will be of iscending order in $h$.

We shall now show inow the solution for the generil case when the given normal traction is a function of $x$, $y$ of unspecificel form may le transformed, under certain restrictions, so as to yield the invintages to which we hive been refuring as pertaining to the solution ( $3: 3),(34)$.

The problem we suppose to be the silluc as that stited at the beginning of $\$ 10$, 
but with $f(x, y)$ instead of $\mathrm{J}_{m} \beta_{\rho} \cos m(w$, and with any continuous area $\mathrm{A}$ instead of the circle within $\rho=a$.

The solution will then be of the form defined in (29), (30), but the integral of (29) will now be

and the integral of $(30)$

$$
\mathbf{F}=\iint \chi(\mathbf{R}) f\left(x^{\prime}, y^{\prime}\right) d x^{\prime} d y^{\prime}
$$

$$
1=\iint \mathrm{r}_{0}(\kappa \mathrm{R}) f\left(x^{\prime}, y^{\prime}\right) d x^{\prime} d y^{\prime}
$$

Since $\Gamma^{2} \chi(R)=\log (R / \underline{m} h)$ we have

also

$$
\left.\begin{array}{c}
\nabla^{4} \mathrm{~F}=\nabla^{2}\left(\nabla^{2} \mathrm{~F}\right)=2 \pi f(x, y) \\
\left(\Gamma^{2}+\kappa^{2}\right) \mathrm{I}=-3 \pi f(x, y)
\end{array}\right\} .
$$

If $f(x, y)$ and its derivatives of the first two orders are finite within $\mathrm{A}$, we may transform I by Green's Theorem. Thus, excluding from the area A an infinitesimal circle about $(x, y)$ as centre, and writing $\nabla^{\prime \prime 2}$ for $\frac{d^{2}}{d x^{2}}+\frac{d^{2}}{d y^{\prime \prime 2}}$,

or

$$
\mathrm{I}=-\frac{1}{\kappa^{2}} \iint f\left(x^{\prime}, y^{\prime}\right) \nabla^{\prime 2} \mathrm{G}_{0} \mathrm{R} d x^{\prime} d y
$$

$$
\begin{aligned}
\mathrm{I}= & -\frac{1}{\kappa^{2}} \iint \mathrm{G}_{0} \kappa \mathrm{R} \nabla^{\prime \prime 2} f\left(x^{\prime}, y^{\prime}\right) d x^{\prime}, l y^{\prime} \\
& -\frac{1}{\kappa^{2}} \int\left\{f\left(x^{\prime}, y^{\prime}\right) \frac{d}{d n}\left(\mathrm{~A}_{0} \kappa \mathrm{R}-\mathrm{G}_{0} \kappa \mathrm{R} \frac{d}{d n} f\left(x^{\prime}, y^{\prime}\right\} d s\right\}\right. \\
& -\frac{2 \pi}{\kappa^{2}} f(x, y)
\end{aligned}
$$

the line integral being taken round the boundary of A.

If this three-termed equivalent of the integral $I$ be substituted in the series for $\phi_{2}$ and $\theta_{2}$, each of these series may be subdivided into three, $\phi_{2}$ for instance into

$$
\sum_{\kappa} \frac{1}{\kappa^{2}} \frac{\cosh \kappa \kappa h \sinh \kappa z}{\kappa h(\cosh 2 \kappa h-1)} \iint \mathrm{C}_{0} \kappa \mathrm{R} \nabla^{\prime 2} f\left(x^{\prime}, y^{\prime}\right) d x^{\prime} d y^{\prime},
$$

a series of the same general form as the original series, but at once more convergent, and of two orders higher in $h$;

$$
\sum_{\kappa} \frac{1}{\kappa^{2}} \frac{\cosh \kappa h \sinh \kappa: \dot{s}}{\kappa h(\cosh 2 \kappa h-1)} \int\left\{f\left(x^{\prime}, y^{\prime}\right) \frac{d}{d n} \mathrm{G}_{0} \kappa \mathrm{R}-\left(i_{0} \kappa \mathrm{R} \frac{\prime \prime}{d n} f\left(x^{\prime}, y^{\prime}\right)\right\} d *,\right.
$$

which corresponds to a strain local to the boundary of $\mathrm{A}$;

(iii) $2 \pi f(x, y) \sum_{\kappa} \frac{1}{\kappa^{2}} \frac{\cosh \kappa h \sinh \kappa}{\kappa(\cosh 2 \kappa l-1)}$, a series which can be summerl in the same way as (31), being in fact simply the first series of $(31)$ with $\beta=0$. The sum is therefore

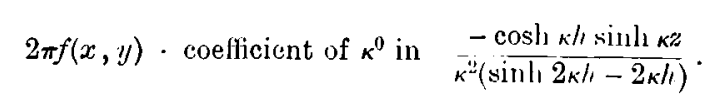

We may now, by repetition of the same transformation, obtain a similar threefold 
equivalent for the series (i), and continue the proress as far as the continuity of the derivatives of $f(x, y)$ will permit. We shonld thus obtain, in place of $\phi_{2}$,

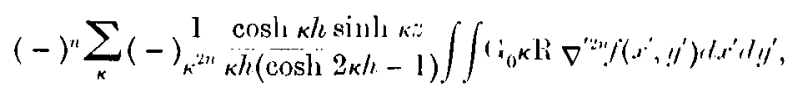

a function of order $h^{2 n+\cdots}$.

(ii) A series of line-intenrals which wre neer not writr slown, corresponding to a local perturbation at the alge of $A$, and giving the arlge values of the relative part of $\phi$ up to terms in $l i n$.

(iii) $\mathrm{A}$ series of $"$ ter'ms

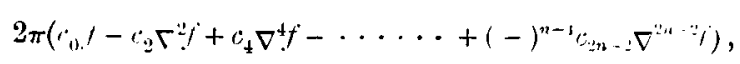

where $c_{3 r}$ is the coefticient of $\kappa^{-2 r}$ in the ascending power uxpullion of $(-) \frac{\cosh \kappa h \sinh \kappa z}{\kappa^{2}(\sinh 2 \kappa h-2 \kappa h)}$, and is obviously a rational integral function of $z$ and $h$ of $1 \operatorname{leg} w \cdot 2 r+2$. If the function $f(x, y)$ his its derivatives of every finite order continuous throughout the area $\mathrm{A}$, the process can be carried to as high a value of $n$ as we please, and we can thus obtain the values of $\phi_{2}, \theta_{2}$ to any required order in $h$. It should be noticerl, however, that the series (iii) is not necessarily convergent when continued to infinity, as we may see by taking as an example $f=\cos \alpha x^{\prime}$, when the series would become $: \pi \cos a x\left(c_{0}+c_{0} t^{2}+c_{4} a^{4}+\cdots\right)$, which is the expansion (without the terms of negative degree) of

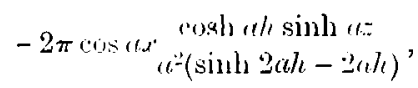

and is therefore divergent if $|2 \mu h|>\left|\zeta_{1}\right|, \zeta_{1}$ being the eromplex root of $\sinh \zeta-\zeta=0$ with smallest modulus. The form of the condition suggrests that in ordinary cases the series will be convergent if $l$ is small enough: and when this is so, this part of $\phi_{2}, \theta_{2}$ taken along with $\phi_{1}, \theta_{1}$ will define an exact particuliur solution within $\Lambda$, giving the proper vilues of the surface tractions, and arranged in terms of ascending order in $h$.

As a special case, the series will terminate if, for some finite value of $n, \nabla^{* n} f=0$, and in particular if $f$ be a rational integral function of $r^{\prime}, y$. (It may be noted here that the solution for $f=\rho^{m+2 p} \cos m \omega$ might be obtained from the solution for $f=\mathrm{J}_{m} \beta \rho$ cos $m \omega$ by expanding in powers of $\beta$, and equating netticients of $\beta^{m+2 p}$ in conditions and solution.)

Looking back now to the $\phi_{1}, \theta_{1}$ part of the solution, and having regard to (18), (29), (35), we see that we may write symbolically

$$
\begin{aligned}
\mathrm{F} & =2 \pi \Gamma^{-4} f^{\prime} ; \nabla^{*} \mathrm{~F}=2 \pi \Gamma^{* \prime \prime} f, \quad \text { and } \\
\phi_{1} & =2 \pi\left(c_{-4} \Gamma^{-1} f-c_{-2} \Gamma^{-2} f\right) .
\end{aligned}
$$

The particular solution to any order in $h$ is then given $b$

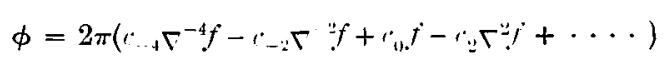

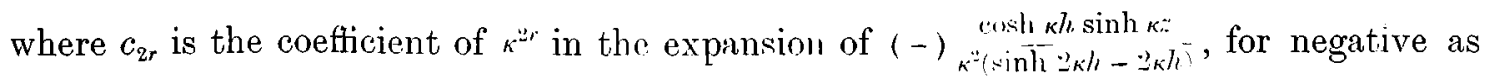
well as positive values of $r$; or, as we may put it, this particnlar part of $\phi$ is given by

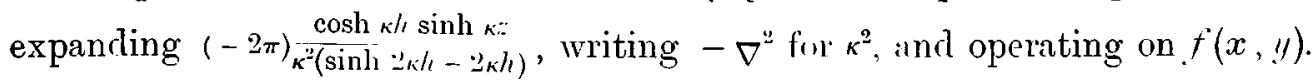




\section{Independent symbolical solution of the yeneral problem.}

The form of the last result suggests a method of dealing with the problem from the beginning, which, though not easy to develop independently with thorough rigour, has the advantage of conciseness, and will therefore be useful in giving a rapid account both of the foregoing solution and of those to be obtained in the following pages.

We begin by observing that

$$
\begin{aligned}
\left(\begin{array}{l}
d^{2} \\
d^{2} x^{2}
\end{array}+\frac{d^{2}}{d^{2} y^{2}}+\frac{d^{2}}{d z^{2}}\right) \sinh \kappa z f(x, y) & =\sinh \kappa\left(\frac{d^{2}}{d x^{2}}+\frac{d^{2}}{d ! y^{2}}+\kappa^{2}\right) f(x, y) \\
& =\sinh \kappa \cdot\left(r^{2}+\kappa^{2}\right) f(c, y) .
\end{aligned}
$$

Hence $\sinh \kappa f(x, y)$ is a potential function, provided we regard $\kappa$ as an operator such that $\kappa^{2}=-\nabla^{2}$. We may, if we please, take $\kappa=i \nabla$, but it will not be necessary to interpret odd powers of the operator $\nabla$.

On this understanding, it is obvious from (12), (13) that we obtain a solution giving

by taking

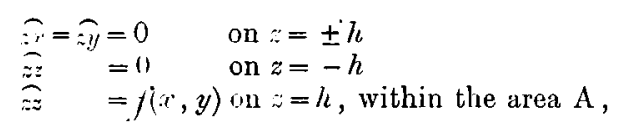

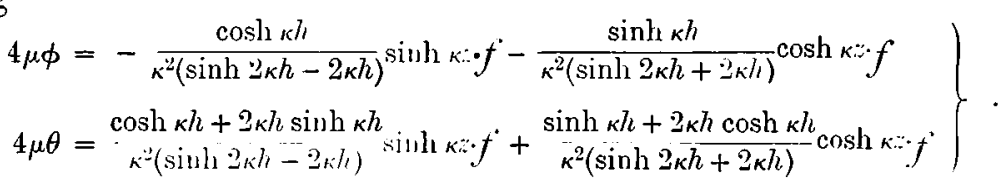

Now, taking as il specimen the first term of $4 \mu \phi$, we observe that the function of $\kappa$

$$
\text { (-) } \frac{\cosh \kappa h}{\kappa^{2}(\sinh 2 \kappa h-2 \kappa h)} \sinh \kappa
$$

vanishes at infinity round the path W A BE of 5 .

Hence the function is represented by the sum of its polar elements. (If $\kappa_{1}$ be a simple pole of the function, and if in the vicinity of this pole the function $=A_{1} /\left(\kappa-\kappa_{1}\right)+$ finite, then $A_{1} /\left(\kappa-\kappa_{1}\right)$ is the polar element at this pole, and $A_{1}$ is the residue there. The point $\kappa=0$ is a multiple pole, and the polar element there has the form $1 / \kappa^{4}+B / \kappa^{2}$, these being the terms of negative degree in the expansion of the function near $\kappa=0$ ).

Taking the elements belonging to $\pm \kappa_{1}$ tomether we obtain

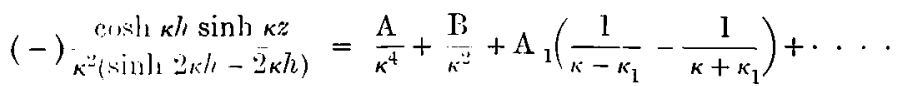

$$
\begin{aligned}
& =\frac{A}{\kappa^{4}}+\frac{B}{\kappa^{2}}+\frac{2 A_{1} \kappa_{1}}{\kappa^{2}-\kappa_{1}{ }^{2}}+\cdots \ldots . \\
& =\frac{1}{\kappa^{4}}+\frac{\mathrm{B}}{\kappa^{2}}+(-) \frac{\cos h_{1} \kappa_{1} h \sinh \kappa_{1}:}{\kappa_{1} h\left(\cosh 2 \kappa_{1} h-1\right)} \frac{1}{\kappa^{2}-\kappa_{1}^{2}}+\ldots \ldots
\end{aligned}
$$

the series extending over the poles with positive imminury part. When we put $\kappa^{2}=-\nabla^{2}$, this part of $4 \mu \phi$ becomes

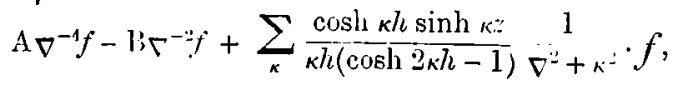

where $\kappa$ is no longer an operator, but simply a root of $\sinh : \kappa^{\prime} h-2 \kappa h=0$. 
Now since

one vilue of

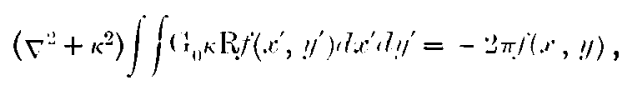

Similarty one

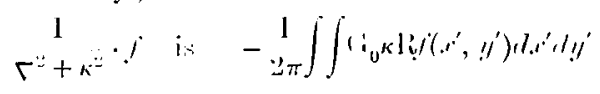

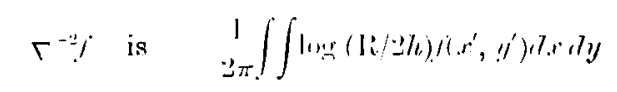

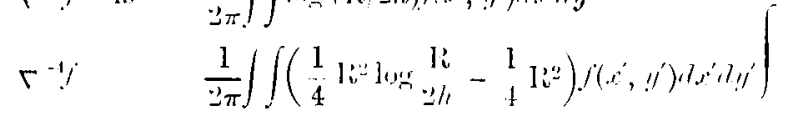

and of

Hene for the first part of $4 \mu \phi$ we olitain

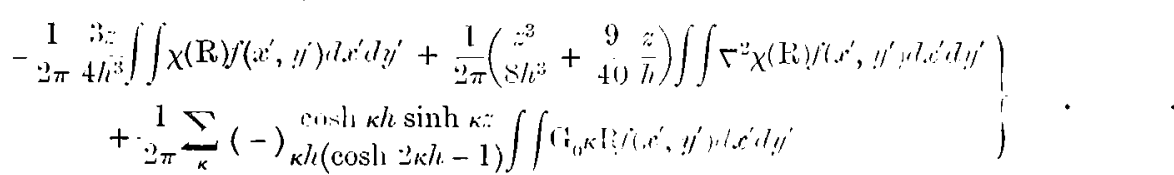

which agrees with our previous solution, the element of whis is given in $(\because 0)$.

Further, the results obtained at the end of $\$ 1+$ clearly agree with what we should get by expanding the function of $\kappa$ in (37) in asending power series and interpreting.

As an example of this use of equations (37) we may find to a first approximation the value of $a$ at points not very close to the edige of 1 .

From (5)

Thus

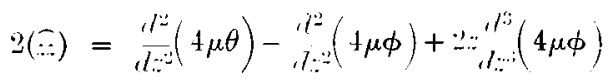

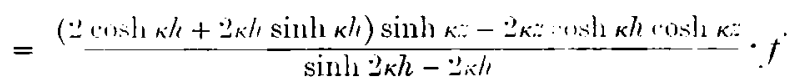

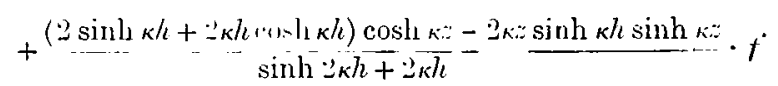

$$
\begin{aligned}
& =\left(3 / i^{2}-z^{2}\right): f l^{3} / l^{3}+f .
\end{aligned}
$$

$$
\Xi=\left\{\left(3 l^{2}-z^{2}\right)=,+1 l^{3}+1,2\right\} f(r,, l)
$$

and we verify at a glance that this gives the proper values at the faces.

16. The problem of tang!ntial fice traction. Solution fir an dement of traction.

We will now pass to the problem in which the given surface traction is tangential. Taking the direction of the traction parallel to the axis of $x$, we may talie for conditions

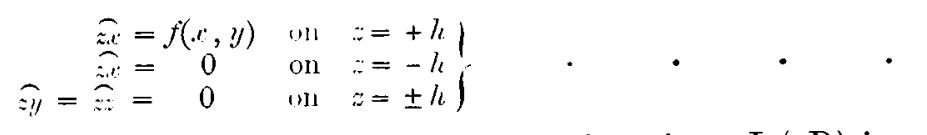

According to the method explained in $\S 3$, we begin with the function $\kappa J_{0}(\kappa R)$ in place of $f(x, y)$, and determine potentials $\psi, \theta, \phi$ giving

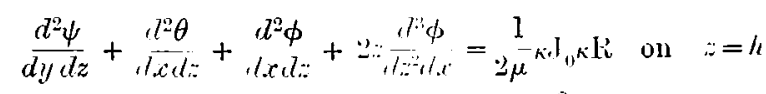

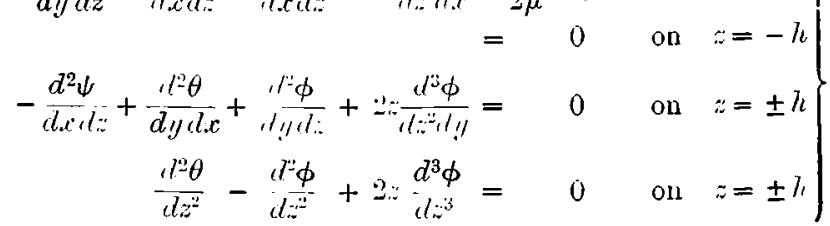

TRANS. ROY. SOC. EDIN., VOL. XLI. PART I. (NO. 8). 


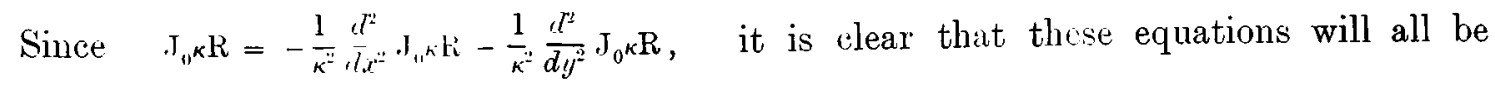
satisfied if we talie

where

$$
\psi=\frac{d \psi^{\prime}}{d ! !}, \theta=\frac{d \theta^{\prime}}{d l^{\prime}}, \phi=\frac{d \phi^{\prime}}{d x} .
$$

From (44),

$$
\begin{aligned}
& \left.\begin{array}{rlrl}
\frac{d \psi^{\prime}}{d t:} & =-\frac{1}{2 \mu} \frac{1}{\kappa} \mathrm{J}_{0} \kappa \mathrm{R} & \text { on } & z=h \\
& =0 & \text { on } & z=-h
\end{array}\right\}
\end{aligned}
$$

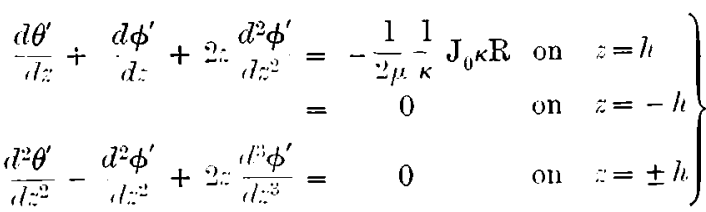

or, separating the odd and eren parts in $z$,

$$
4 \pi \psi^{\prime}=-\frac{1}{\kappa^{2}} \frac{\cosh \kappa: 2}{\sinh \kappa h} \mathrm{~J}_{0^{\circ}} \kappa \mathrm{R}-\frac{1}{\kappa^{2}} \frac{\sinh \kappa}{\cosh \kappa h} \mathrm{~J}_{0^{\prime}} \kappa \mathrm{R}
$$

We also find easily

$$
\begin{aligned}
4 \pi \phi^{\prime}= & -\frac{\sinh \kappa h}{\kappa^{2}(\sinh 2 \kappa h-2 \kappa h)} \mathrm{J}_{v^{\kappa}} \mathrm{R} \sinh \kappa z \\
& -\frac{\cosh \kappa h}{\kappa^{2}(\sinh 2 \kappa h+2 \kappa h)} \cdot \mathrm{J}_{0} \kappa \mathrm{R} \cosh \kappa \% \\
4 \mu \theta^{\prime}= & \frac{2 \kappa h \cosh \kappa h-\sinh \kappa h}{\kappa^{\prime \prime}(\sinh 2 \kappa h-2 \kappa h)} \mathrm{J}_{v^{\kappa}} \mathrm{R} \sinh \kappa z \\
& +\frac{2 \kappa h \sinh \kappa h-\cosh \kappa h}{\kappa^{2}(\sinh 2 \kappa h+2 \kappa h)} \mathrm{J}_{0^{\kappa}} \mathrm{R} \cosh \kappa *
\end{aligned}
$$

Treating these expressions as in $\$ 3$, we find a solution for an element of $\mathrm{X}$-traction at $\left(x^{\prime}, y^{\prime}, h\right)$ of $8 \pi \mu$ units given by $(43)$ with

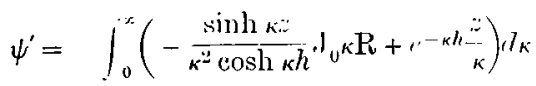

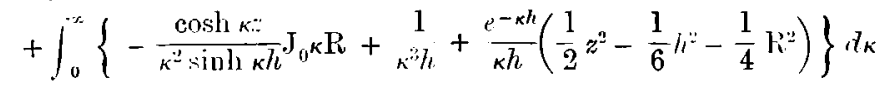

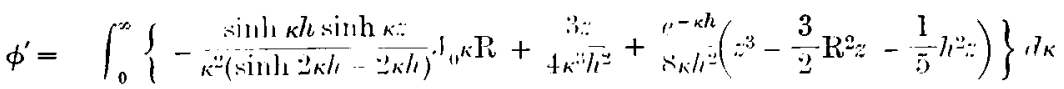

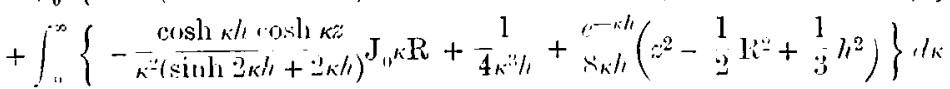

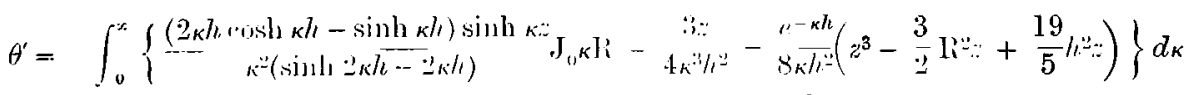

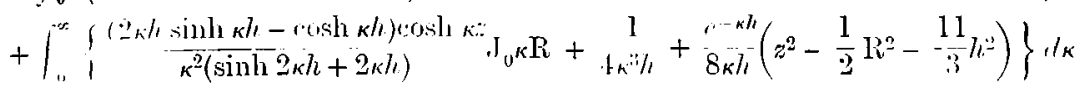

These expressions may he trins linmed by the method of $\$ 5$, and a slight inspection of the relations between (16), (17) and (20), (21) will cnable us to write down the 


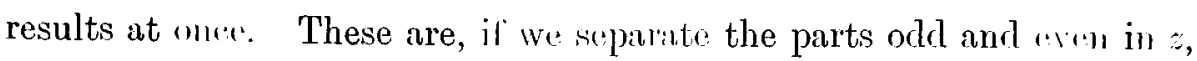

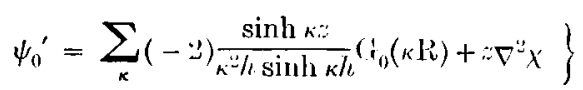

where $\kappa$ is a positive imaginary root of enshekh,

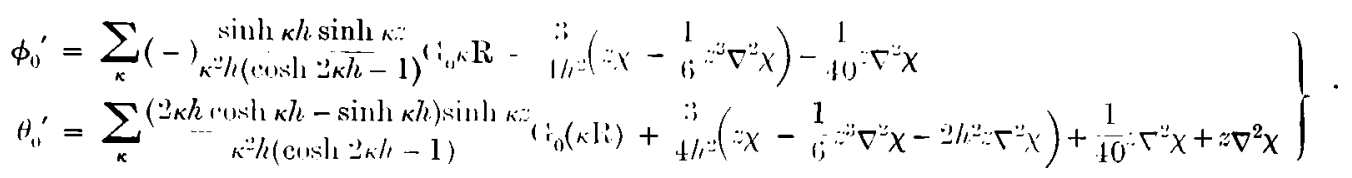

where $\kappa$ is a zero of $\sinh : 2 k-{ }^{2} \kappa h$, with positive imaginary part.

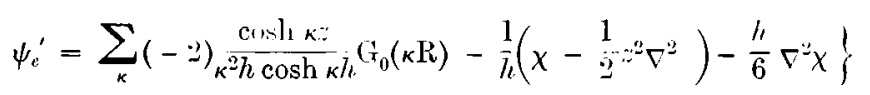

where $\kappa$ is a positive imaginary root of $\sinh \kappa h$.

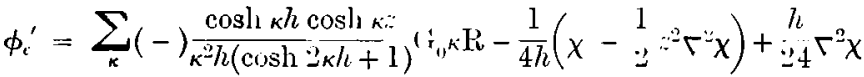

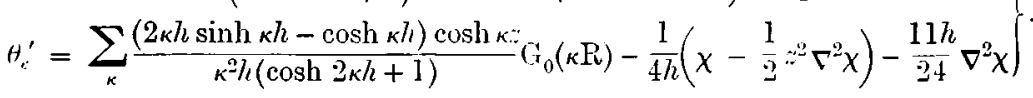

where $\kappa$ is a zero of $\sinh \beth^{\prime} h+\vartheta_{\kappa} k$, with positive imaginary part.

The solution is defined by these equations with

$$
\psi=\frac{d \psi^{\prime}}{d ! !}, \theta=\frac{d \theta^{\prime}}{d x}, \phi=\frac{d \phi^{\prime}}{d x} .
$$

\section{Compusition of the solution.}

On examining the composition of the solution, we observe in the decaying parts of $\phi, \theta$, solutions of the class already obtained in ( 22$),(\because 5)$, and in the corresponding part of $\psi$, solutions of the type

$$
\begin{aligned}
& \psi=\sin (2 n+1) \frac{\pi \cdot:}{2 h} \mathrm{~F}(x, y) \\
& \psi=\cos \frac{n \pi z}{h} \mathrm{~F}(r, y),
\end{aligned}
$$

each giving $\frac{d \psi}{d:}=0$ on $z= \pm h$, and therefore zero tractions at the surface. As for the permanent terms, they may be arranger in the following groups, in each of which the surface stresses vanish.

$$
\begin{aligned}
& \left.\phi=-\frac{3}{4 h^{2}} \quad\left(x-\frac{1}{6}\right)^{3} \Gamma^{2} x\right)
\end{aligned}
$$

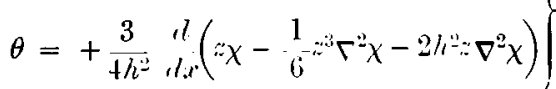

$$
\begin{aligned}
& \phi=-\frac{1}{40}: \frac{d}{d x} \Gamma^{2} \chi \\
& \left.\theta=\frac{1}{40} z \frac{d}{d x} \nabla^{2} x\right\}
\end{aligned}
$$


These are of the types (29), $\because 4)$.

(iii)

$$
\left.\begin{array}{rl}
\psi & =z \frac{d}{d ! l} \nabla^{2} x \\
& =z \frac{d}{d, r^{2}} \nabla^{2} x
\end{array}\right\}
$$

This gives, by (4), since $\nabla^{4} \chi=0$,

$$
\begin{aligned}
& t=z^{2} \frac{d^{2}}{d y^{2}} \nabla^{2} x=-\pi \cdot \frac{l^{2}}{d x x^{2}} \nabla^{2} x \\
& n=-i \frac{d^{2}}{d x d y} \nabla^{2} \chi \\
& n=\frac{\pi}{7 l} \Gamma^{2} \chi
\end{aligned}
$$

and may therefore be considered as of the type (24). It is important to note, however, both here and in the cases immediately following, that the transformation on the value of " will not hold after the elementary solution has been integrated for the purposes of the general problem in $( \pm 1)$.

$$
\begin{aligned}
& \psi=-\frac{1}{h} \cdot \frac{d}{d !}\left(x-\frac{1}{2} \cdot{ }^{2} \Gamma^{2} x\right) \\
& \left.\phi=-\frac{1}{4 h} \frac{d}{d \lambda}\left(x-\frac{1}{2}=\nabla^{2} x\right)\right\} \\
& \left.=-\frac{1}{4 h} \frac{1}{d n^{2}}\left(x-\frac{1}{2}\right)^{2} \Gamma^{2} x\right)
\end{aligned}
$$

Now $F$ being any function of $x$, y, satisfying $\nabla^{4} \mathrm{~F}=0$, the solution

leads to

$$
\begin{aligned}
\psi & =4 \frac{d}{d !}(\mathrm{F}-1 \\
\dot{\phi}=\theta & =\frac{d}{d x}\left(\mathrm{~F}-\frac{1}{2} \nabla^{2} \mathrm{~F}\right)
\end{aligned}
$$

$$
\begin{aligned}
& u=\quad s \frac{d^{2} \mathrm{~F}}{d y^{2}}+(a+1) \frac{d^{2} \mathrm{~F}}{d x^{2}}+\frac{3-a^{2}}{z^{2}} d d^{2} r^{2} \Gamma^{2} \mathrm{~F}
\end{aligned}
$$

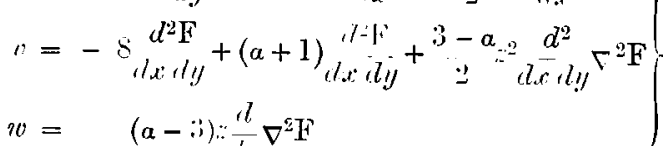

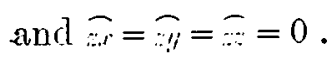

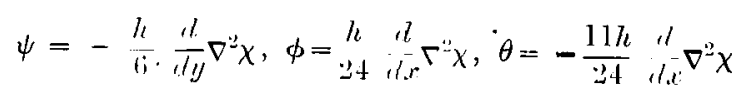

which may be further decompesed into

of the form $(26)$, ami

$$
\psi=0, \phi=\frac{l 1}{2-4} \frac{d}{d x} \nabla^{2} \chi, \theta=-\frac{h}{8} \frac{d}{d x} \Gamma^{2} \chi,
$$

$$
\psi=-\frac{h}{6} \frac{d}{d ! y} \nabla^{2} \chi, \phi=0, \theta=-\frac{h}{3} \frac{d / 3}{d x} \nabla^{2} \chi,
$$

the displacements rore-ponling to which vanish. 


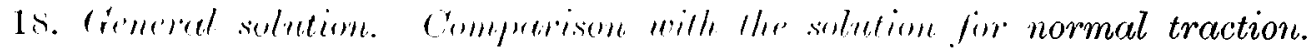

The solution of the seneral problem of $(+1)$ may lo: found by multiplying the expressions for $\psi^{\prime}, \theta^{\prime}, \psi^{\prime}$ in (18) . . . (51) by $\frac{1}{8 \pi \mu} f\left(r^{\prime}, l^{\prime}\right)$ : inl integrating term by term over the area 1 within which $f$ is finite. Is in the case of the problem of given normal triction, term ly term lifferentiations of the resulting series will be legitimate just so far as the derivatives are required for the calculation of displacements and stresses. In order to see this, it should he noticed that, while an extra differentiation as to ${ }^{\prime}$ or $y$ will be required in virtue of $(43)$, the series for $\psi^{\prime}, \theta^{\prime}$. $\phi^{\prime}$ have general terms of one order higher in $1 / \kappa$ as compared with thos: of (20), (21). One effect, however, of this alditional differentiation will be to increase the relative importance at the edge of the area $A$ of that part of the displacement and stress which arises from the local perturhition, such displacement hing of order $h$, amil stress of order zero, as in the former problem, whereas the displacement and stress as a whole is of higher order in $h$ than before.

The functions $\psi^{\prime}, \theta^{\prime}, \phi^{\prime}$ being symmetrical about the axis $R=0$, it is clear that the solution for in element of traction of $8 \pi \mu$ units at $\left(x^{\prime} \cdot y^{\prime}, l\right)$ parallel to the axis of $y$ is given by

$$
\psi=-\frac{d \psi^{\prime}}{d, r}, \theta=\frac{d \theta^{\prime}}{d ! l}, \phi=\frac{l l \phi^{\prime}}{d ! !}
$$

with $\psi^{\prime}, \theta^{\prime}, \phi^{\prime}$ is in (18)... (51).

It will be seen presently that surface traction may lie regarled as a special case of force applied in the body of the plate. We may therefore postpone any more extended development of the above solution, and in particular ally more explicit comparison of the results with those of the accepted approximate theory of thin plates, until we have obtained the solutions of tho problems relative to sources of strain situated in the interior of the solid.

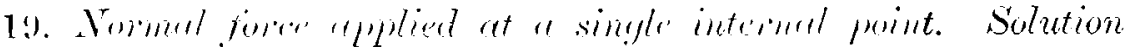 in definite integrals.}

W. take first the cus of a single force, say for convenience of $t \pi \mu(\alpha+1)$ units, applied at $\left(x^{\prime}, y^{\prime}, y^{\prime}\right)$ parallel to $(y)$, the lines of the plate being free from stress. Referring to (6), we see that the conditions of the problem may be taken to be

$$
\begin{aligned}
& u=\left(i^{\prime}-\because\right)^{d r^{-1}} \frac{d r}{d t}
\end{aligned}
$$

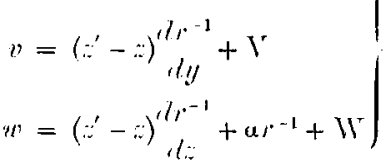

(ii) $\mathrm{U}, \mathrm{V}, \mathrm{W}$, along with their lerivatives as to $(x, y, z)$ of the first order, are finite and continuous at erery point of the solid at a finite distance, and have derivatives of 
the second order satisfying

where

$$
\left.\mu \nabla^{2} \mathrm{U}+(\lambda+\mu)^{\prime \prime \Delta} \frac{d \Delta}{d x}=0 ; \mu \nabla^{2} \mathrm{~V}+(\lambda+\mu)^{\prime \prime \Delta} \frac{d \Delta}{d y}=0 ; \mu \Gamma^{2} \mathrm{TV}+(\lambda+\mu)^{\prime \prime}\right)^{\prime \Delta}=0 ;
$$

(iii) $\widehat{z x}=\widehat{-i y}=\widehat{\because:}=0$ on $z= \pm h$

$$
\mathrm{A}=\frac{d \mathrm{U}}{l d x}+\frac{d \mathrm{~V}}{\mathrm{ll} !}+\frac{d \mathrm{~W}}{d ! 2}
$$

It is clear that these conditions do not completely define the solution, seeing that no condition to be satisfied at infinity is mentioned. But instead of laying down any such condition at infinity, it is simpler in the first instance to be content with any solution fulfilling (i), (ii), and (iii). The most general solution can then be obtained without difficulty, and with this before us, conditions at infinity can be riscussed to much greater advantage than at present.

The problem is solved when $U, V, W$ are found in the form (4), so as to give the same tractions on $z= \pm h$ as those due to (6), but reversed. These reversed tractions, as follows very readily from (5), (8), are given by

Now when $z>:^{\prime}$,

$$
\begin{aligned}
& \begin{array}{l}
\frac{\partial y}{\partial \mu}=\frac{d}{d x} \mid\left(\frac{1-a}{2} r^{-1}+\left(z-z^{\prime}\right) \frac{d r^{-1}}{d z} \mid\right. \\
\frac{\hat{z} y}{\partial \mu}=\frac{d !}{d ! \lambda} \mid
\end{array} \\
& \widehat{\hat{\overline{2}}}=\quad-\frac{1+a}{2} \frac{d r^{-1}}{d z}+\left(z-z^{\prime}\right)^{\prime l^{2} r^{-1}} \frac{z^{2}}{d z^{2}}
\end{aligned}
$$

but when $z<z^{\prime}$,

$$
\begin{aligned}
& r^{-1}=\int_{0}^{\infty} e^{-\kappa\left(z-z^{\prime}\right)} \mathrm{J}_{0} \kappa \mathrm{R} d \kappa \\
& \frac{d r^{-1}}{d z}=\int_{0}^{\infty}(-\kappa) e^{-\kappa\left(z-z^{\prime}\right) \mathrm{J}_{0} \kappa \mathrm{R} d \kappa} \\
& \frac{d r^{\prime} r^{-1}}{d r^{2}}=\int_{0}^{\infty} \kappa^{2, \nu^{\prime}} \kappa\left(z-z^{\prime}\right) \mathrm{J}_{0} \kappa \mathrm{R} d \kappa
\end{aligned}
$$

$$
\begin{aligned}
& r^{-1}=\int_{0}^{\infty}{ }^{r} \kappa\left(z-z^{z}\right) \mathrm{J}_{0} \kappa \mathrm{R} d \kappa \\
& \frac{d r^{-1}}{d z}=\int_{0}^{\infty} \kappa \kappa \kappa(z-z) \mathrm{J}_{0} \kappa \mathrm{R} d \kappa \\
& \frac{d t^{2} r^{-1}}{d z^{2}}=\int_{0}^{\infty} \kappa^{2} e^{\kappa\left(z-z^{\prime}\right) \mathrm{J}_{0} \kappa \mathrm{R} d \kappa}
\end{aligned}
$$

Hence if $U, V, W$ be defined as in (4), the function $\psi$ is not requirul, and the conditions to be satisfied by $\theta, \phi$ are, if in the first instance wr take integrands instead of integrals,

$$
\begin{aligned}
& \frac{d \theta}{d z}+\frac{d \phi}{d z}+2 z \frac{d^{3} \phi}{d z^{2}}=\left\{\frac{1-a}{2}-\kappa\left(h-z^{\prime}\right)\right\} e^{-\kappa\left(h-\ddots^{\prime}\right), J_{0} \kappa} \mathrm{R}, \text {, wn } z=h \\
& =\left\{\begin{array}{c}
1-a \\
2
\end{array}-\kappa\left(l+z^{\prime}\right)\right\} e^{-\kappa\left(h+z^{\prime}\right)} \mathrm{J}_{0} \mathrm{~K} \mathrm{R} \text {, on } z=-h \\
& \frac{d^{2} \theta}{d z^{2}}-\frac{d^{2} \phi}{d z^{2}}+2 z^{\cdot l^{3} \phi} \frac{1+a}{d \cdot z^{3}}=\left\{\frac{1+a}{2} \kappa+\kappa^{2}\left(h-z^{\prime}\right)\right\} e^{-\kappa(h-z)} \mathrm{J}_{0} \kappa \mathbf{R} \text {, on } z=h \\
& =\left\{-\frac{1+a}{2} \kappa-\kappa^{2}\left(h+z^{\prime}\right)\right\}^{--\kappa\left(h+z^{\prime}\right) J_{0} \kappa R, \text { on } z=-h .} .
\end{aligned}
$$


Assmmill:!

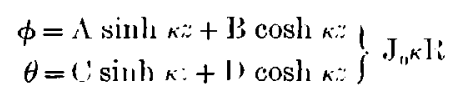

(55) give four aplutions to dedermine A, B, (), T). By addition and subtraction

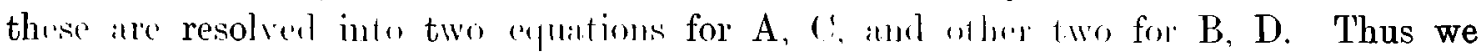
find

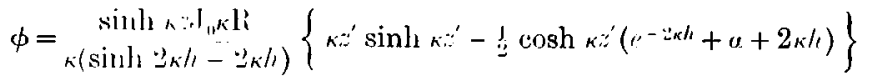

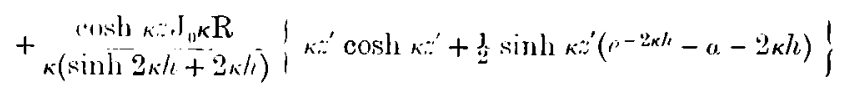

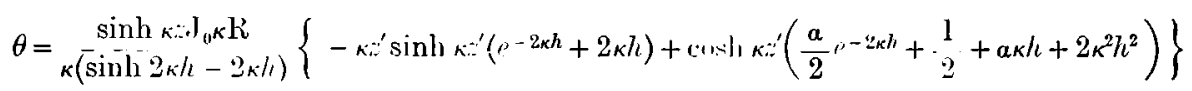

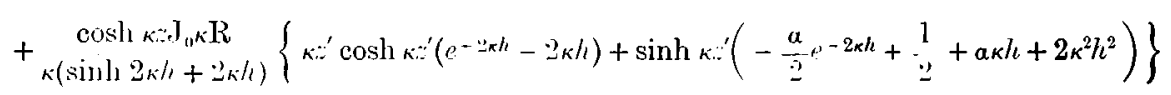

If these expressions could be integraterl with respect to $\kappa$ from 0 to $\infty$, the balancing displacements U, V, W of (55) would be determined. Near the upper limit the functions converge to zero exponentialwise, since both $\approx$ and $z^{\prime}$ lie between $-h$ and $+h$. But for $\kappa=0$ both functions are infinite, and their expansions in ascending powers of $\kappa$ contain terms of negative degree which must be removed after the manner of $\S 3$. The integrals are then convergent, but a further modification of a different sort is necessary before they an be transformed into series as in $\$ 5,16$. The possibility of this transformation in the former cases was intimately related to the fact that the functions in (13), (46) were odd in $\kappa$, which the functions in (58) obviously are not. However, when the odd and even parts are separated, the latter are found to have a very simple form, free from the denominators $\sinh 2_{k} h \pm 2 k h$, for we find

$$
\begin{aligned}
& \phi=\frac{1}{2 \kappa} \sinh \kappa\left(:-x^{\prime}\right) \cdot \mathrm{J}_{\mathrm{v}} \mathrm{K} \mathrm{R}
\end{aligned}
$$

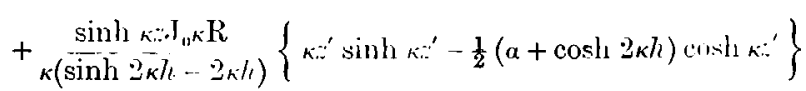

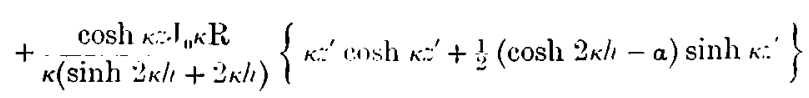

$$
\begin{aligned}
& \theta=-\left\{\frac{a}{2 \kappa} \sinh \kappa\left(z-z^{\prime}\right)+z^{\prime} \cosh \kappa\left(z-z^{\prime}\right)\right\} \mathrm{J}_{0} \kappa \mathrm{K}
\end{aligned}
$$

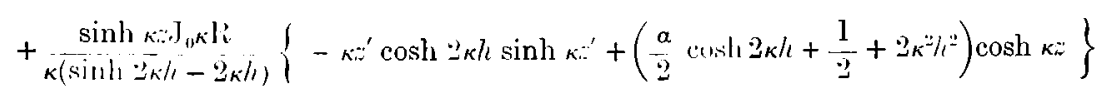

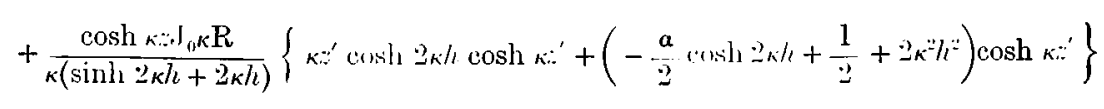

The even terms in $n$ can he eliminated from these "xpressions by including the 
values of $\phi, \theta$ which define the source, given in (8). For these are

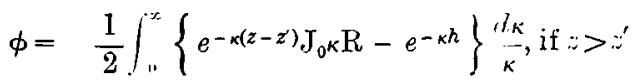

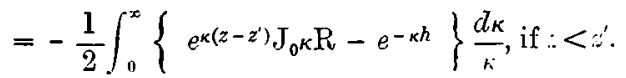

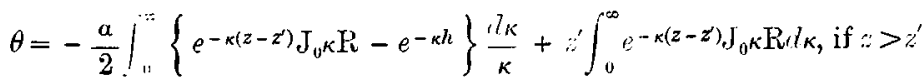

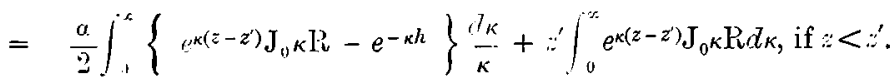

These will obviously be reproduced, after preparation by removal of terms of negative degree and integration, from

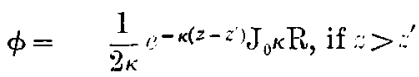

$$
\begin{aligned}
& =-\frac{1}{2 \kappa} \kappa^{\kappa(z-z) J_{0} \kappa} \mathrm{R}, \text { if } z<z^{\prime} . \\
& \theta=\left(-\frac{a}{2 \partial_{\kappa}}+z^{\prime}\right) e^{-\kappa(z-z) \mathrm{J}_{0} \kappa} \mathrm{R}, \text { if }: i>z^{\prime} \\
& =\left(\frac{a}{2 \kappa}+z^{\prime}\right) e^{(z-z)} \mathrm{J}_{n} \kappa \mathrm{R} \text {, if } z<i^{\prime} .
\end{aligned}
$$

When these last terms are taken in, the first lines of $\phi, \theta$ in (59) become

$$
\begin{aligned}
& \phi= \pm \frac{1}{2 \kappa} \cosh \kappa\left(:-z^{\prime}\right) \mathrm{J}_{0} \kappa \mathrm{R} \\
& \theta=\mp \frac{a}{2} \cosh \kappa\left(: \because-z^{\prime}\right) \mathrm{J}_{0} \kappa \mathrm{R} \mp z \sinh \kappa\left(:-z^{\prime}\right) \mathrm{J}_{0} \kappa \mathrm{R},
\end{aligned}
$$

the upper or lower sign being taken in the ambiguities according as $z\rangle$ or $\left\langle z^{\prime}\right.$. Hence, when the source is taken in, the following are the unprepared and unintegrated forms of $\phi, \theta$ :-

$$
\begin{aligned}
& \phi= \pm \frac{1}{2 \kappa} \cosh \kappa\left(:-z^{\prime}\right) \mathrm{I}_{0^{\kappa}} \mathrm{R}
\end{aligned}
$$

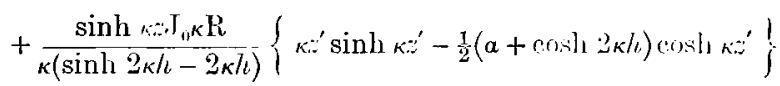

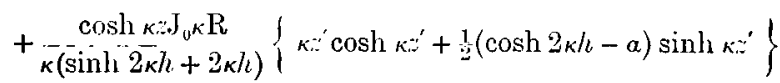

$$
\begin{aligned}
& \theta=\mp \frac{a}{2 \kappa} \cosh \kappa\left(z-z^{\prime}\right) \cdot J_{0} \kappa \mathrm{R} \mp z^{\prime} \sinh \kappa\left(z-z^{\prime}\right) \mathrm{J}_{0} \kappa \mathrm{R} \\
& +\frac{\sinh \kappa z J_{0} \kappa \mathrm{R}}{\kappa(\sinh 2 \kappa h-2 \kappa l \epsilon)}\left\{-\kappa z^{\prime} \cosh \nu^{\prime} h h_{1} \sinh \kappa z^{\prime}+\left(\frac{\alpha}{2} \cosh 2 \kappa h+\frac{1}{2}+2 \kappa^{2} / h^{2}\right) \cosh \kappa \kappa^{\prime}\right\}
\end{aligned}
$$

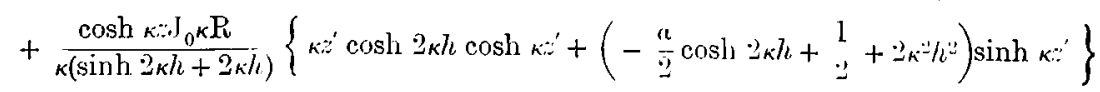

In (61) the terms of negative degree in $\kappa$ are of the forms:-in $\phi, \mathrm{H} / \kappa^{3}+\mathrm{K} / \kappa$; in $\theta, \mathrm{L} / \kappa^{3}+\mathrm{M} / \kappa$; and these terms, as in $\$ 3$, give $\widehat{x}=\widehat{z}=\widehat{z}=0$ at the faces of the plate. 
Hence if from $\phi$ in $(61)$ we subtract $\mathrm{H} / \kappa^{3}+\mathrm{K}_{r^{-\alpha h} / \kappa}$, and from $\theta, \mathrm{I}_{1} / \kappa^{3}+\mathrm{N}_{e^{-\alpha h}} / \kappa$, the resulting expressions, integrated with respert to $k$ from 0 tro $\infty$, will define a solution of the problem stated at the beginning of this article.

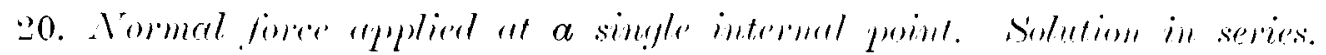

To the integrials thus olitininel we can apply the transformation of $\$ 5$, but one remark slould be mule. From the synthesis which save (6il), it is sufficiently robvious, in riew of the foms in (58) and (60), that the cxpressions of $(61)$, with $\left(\mathrm{A}_{1}, \mathrm{R}\right.$ substituted for $\mathrm{J}_{n}(\kappa \mathrm{R})$, vanish effectively at infinity in the first quarlant of the $\kappa$ plane; that the similarly vinish in the second yuadrant follows at once from the fact that the functions of (61) are odd functions of $\kappa$.

$\therefore$ glance at the relation between (16), (17) and ( 20$),(\because 1)$ will again save us the necessity of writing down the details. Thus, let the values of $H, K, L, M$, when $R$ is put equal to zero, be denoted by $\mathrm{H}_{0}, \mathrm{~K}_{0}, \mathrm{~L}_{0}, \mathrm{M}_{10}$. Then the persistent part of the transformed solution is given by

$$
\left.\begin{array}{l}
\phi=\mathrm{H}_{u} \chi(\mathrm{R})-\mathrm{K}_{0} \Gamma^{*} \chi\left(\mathrm{l}_{i}\right) \\
\theta=\mathrm{L}_{0} \chi(\mathrm{R})-\mathrm{M}_{i j} \Gamma^{-2} \chi\left(\mathrm{Ii}_{i}\right)
\end{array}\right\} .
$$

The decaying part is given by

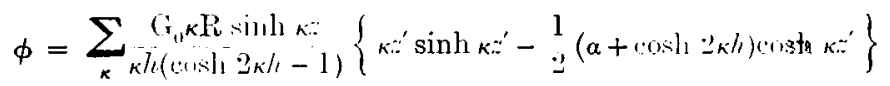

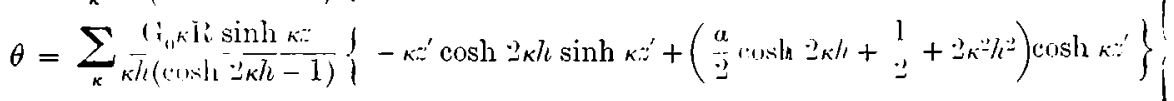

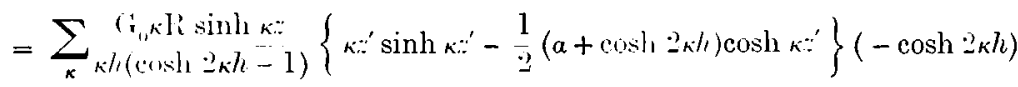

where $\kappa$ is a zero of $\sinh { }^{\prime} k h-2 k h$, with positive imalinally part;

with

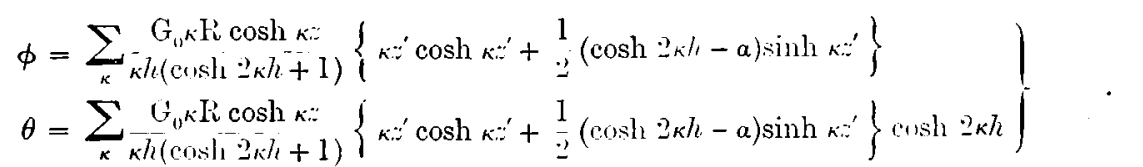

where $\kappa$ is a zero of $\sinh : * h+2 \kappa h$, with positive imasinary part.

When the values of $\mathrm{H}_{0}, \mathrm{~K}_{0}, \mathrm{~L}_{0}, \mathrm{M}_{0}$ are obtained from (6i1), it will be found that (6.) may be decomposed as follows :-

$$
\begin{aligned}
& \phi=-\frac{3(a+1)}{8 h^{3}}\left(\because x-\frac{1}{6} ; \nabla^{3} \nabla^{2} x\right) \\
& \theta=\frac{3(u+1)}{s / u^{-}}\left(z x-\frac{1}{6} z^{3} \nabla^{2} x-2 h \nabla^{2} x\right)
\end{aligned}
$$

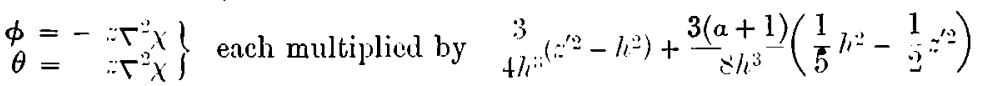

$$
\begin{aligned}
& \phi=\frac{y^{\prime}(a-3)}{8 h} \nabla^{2} X=\theta
\end{aligned}
$$

or less than $z^{\prime}$. $\phi=\mp \frac{1}{2} \Gamma^{2} \chi ; \theta= \pm \frac{1}{2} a \nabla^{2} \chi$, with upper or lower signs, as $z$ is greater

TRANS. ROY. SOC. EDIN., VOL. XLI. PART I. (NO. s). 
There being no discontinuity of displacement or strain at the plane $z=z^{\prime}$, except at the point where the force is ipplied, we are prepared to find that (iv) give no displacement at all if $R>0$. So long. however, as we keep to the specification of the strain by the $\phi, \theta$ functions, it is convenient to retain the terms in (iv). By so doing, we of course make the $\phi$ of the space above the plane $z=z^{\prime}$ and the $\phi$ of the space below that plane two distinct potential functions, but we preserve the non-singular character of each of these functions at the axis $\mathrm{R}=0$.

If we take the limit of the above solution for $z^{\prime}=h$, which obviously may be done by putting $z^{\prime}=h$ in each term and using the lower signs in (iv), we obtain simply the solution of $(20),(21)$ multiplied by $\frac{1}{2}(\alpha+1)$. Since the present solution is for a force of $4 \pi \mu(a+1)$ units, and the other for an element of traction of $8 \pi \mu$ units, it follows that a unit element of traction may be regarded as simply the limiting case of a unit force, the point of application of which approaches indefinitely near the surface.

\section{Solution of a special problem of internal areal normal force.}

When the displacements due to a unit $Z$ force at $\left(x^{\prime}, y^{\prime}, z^{\prime}\right)$, with the surface free, are known, the corresponding displacements for a body distribution of force, of amount $Z\left(x^{\prime}, y^{\prime}, z^{\prime}\right)$ per unit volume at $\left(x^{\prime}, y^{\prime}, z^{\prime}\right)$, can be found by multiplying by $\mathrm{Z}\left(x^{\prime}, y^{\prime}, z^{\prime}\right) d x^{\prime} d y^{\prime} d z^{\prime}$ and integrating through the space in which $\mathrm{Z}$ is finite. Certain peculiarities in the form of the solution given in $\$ 20$ make it convenient to take the integration with respect to $z^{\prime}$ last, or, as comes to the same thing, to begin by considering the solution for an areal distribution of force on the plane $z=z^{\prime}$, of magnitude $Z\left(x^{\prime}, y^{\prime}, z^{\prime}\right)$ per unit area.

We take first a special problem analogous to that worked out in $\$ 10$, and suppose the $\mathrm{Z}$ force to be distributed over the area of a circle of radius $a$ in the plane $z=z^{\prime}$, with centre on $\mathrm{O} z$, the intensity per unit area being $4 \pi \mu(\alpha+1) \mathrm{J}_{m} \beta \rho \cos m \omega$. It will be sufticient to attend to the value of $\phi$, for when that is known, the corresponding value of $\theta$ can be written down at once.

The series deduced by integration from (63), (64), say $\phi_{2}$, fall naturally into two parts as in $\$ 10$, viz., (i) series defining a local perturbation at the cylinder $\rho=a$,

$$
\begin{aligned}
& \phi=\sum_{\kappa} \frac{\sinh \kappa}{\kappa h(\cosh 2 \kappa h-1)}\left\{\kappa z^{\prime} \sinh \kappa z^{\prime}-\frac{1}{2}(a+\cosh 2 \kappa h) \cosh \kappa z^{\prime}\right\} \cdot \frac{2 \pi}{\beta^{2}-\kappa^{2}} \mathrm{P}_{\kappa} \quad(\text { where } \sinh 2 \kappa h-2 \kappa h=0) \\
& +\sum_{\kappa} \frac{\cosh \kappa}{\kappa h(\cosh 2 \kappa h+1)}\left\{\kappa z^{\prime} \cosh \kappa z^{\prime}+\frac{1}{2}(\cosh 2 \kappa h-a) \sinh \kappa z^{\prime}\right\} \cdot \frac{2 \pi}{\beta^{2}-\kappa^{2}} P_{\kappa}(\text { where } \sinh 2 \kappa h+2 \kappa h=0) \\
& \mathrm{P}_{\kappa}=\mathrm{J}_{m, m} \kappa \rho \cos n \omega \omega\left(\kappa a \mathrm{G}^{\prime \prime}{ }^{\prime} \kappa a \mathrm{~J}_{m} \beta a-\mathrm{G}_{n k} \kappa a \beta a \mathrm{~J}_{m}{ }^{\prime} \beta a\right), \quad \text { if } \rho<a \\
& =\mathrm{G}_{m} \kappa \rho^{\prime} \cos m \omega\left(\kappa \omega \mathrm{J}_{m}{ }^{\prime} \kappa a \mathrm{~J}_{m} \beta a-\mathrm{J}_{m} \kappa a \cdot \beta a \mathrm{~J}_{m}{ }^{\prime} \beta a\right) \text {, if } \rho>a
\end{aligned}
$$

(ii) When $\rho<a$, series which can be summed in finite terms,

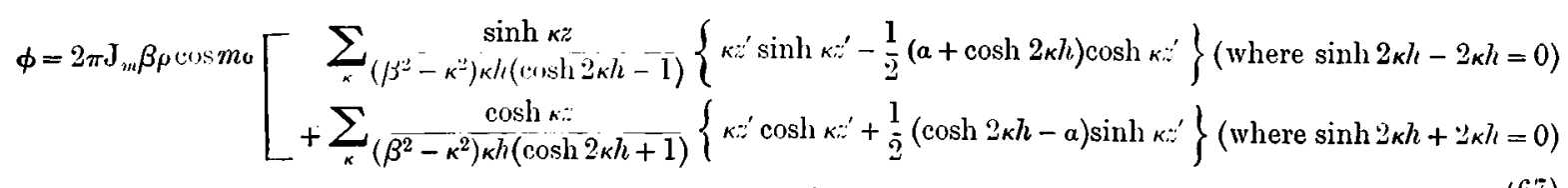


In order to sum these last series, consider the function of "

$$
\begin{aligned}
& \pm \quad \begin{array}{c}
\cos 1 / \kappa\left(z-z^{\prime}\right) \\
2 \kappa\left(\beta^{2}-\kappa^{2}\right)
\end{array} \quad\left( \pm \text { accomding as } z>\text { or }<z^{\prime}\right)
\end{aligned}
$$

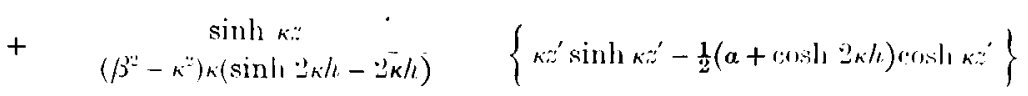

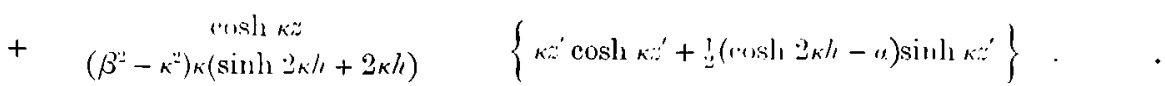

Looking back at (61) we assure ourselves that this function vallishes at infinity in such at wily as to malke the sum of its residues zero. Also, since the function is odd in $\wedge$, the resiclues at $\kappa= \pm \kappa_{1}$ are equal, and therefore the sum of the residues at the zeroes of $\sinh 2 \kappa h \pm 2 \kappa h$ is simply the coefficient of $2 \pi \mathrm{J}_{m} \beta \rho \cos m(n)$ in (67). The sum of the residues at $\kappa= \pm \beta$ is

$$
\begin{aligned}
& \mp \frac{\cosh \beta\left(z-z^{\prime}\right)}{\underline{2} \beta^{\prime 2}} \\
-\frac{\sinh \beta:}{\beta^{2}(\sinh 2 \beta h-2 \beta h)} & \left\{\beta z^{\prime} \sinh \beta z^{\prime}-\frac{1}{2}(\alpha+\cosh 2 \beta h) \cosh \beta z^{\prime}\right\} \\
-\frac{\cosh \beta}{\beta^{2}(\sinh 2 \beta h+2 \beta h)} & \left\{\beta z^{\prime} \cosh \beta z^{\prime}+\frac{1}{2}(\cosh 2 \beta h-a) \sinh \beta z^{\prime}\right\}
\end{aligned}
$$

If this last expression near $\beta=0$ be of the form $\frac{A}{\beta^{4}}+\frac{B}{\beta^{2}}+\ldots$, the residue at $\kappa=0$ of $(68)$ is simply $-\frac{A}{\beta^{4}}-\frac{B}{\beta^{2}}$.

Hence the coefficient of $2 \pi J_{m} \beta \rho \cos m \omega$ in (67) is simply (69) with sign changed and the terms of negative degree in $\beta$ subtracted. These terms of negative degree, just as in $\$ 12$, are added on again when we take in the part of the solution coming from (65), which is obtained by writing $\mathrm{F}$ for $\chi$ in (65) where

$$
\begin{aligned}
\mathrm{F} & =\iint \chi(\mathrm{R}) \mathrm{J}_{m} \beta \rho^{\prime} \cos m \omega^{\prime} \rho^{\prime} d \rho^{\prime} d \omega^{\prime} . \\
& \left.=\frac{2 \pi}{\beta^{4}} \mathrm{~J}_{m} \beta \rho \cos m \omega+\mathrm{F}_{1} . \quad \text { (Introd. }\left(f^{\prime}\right) .\right)
\end{aligned}
$$

The term $\frac{2 \pi}{\beta^{4}} J_{m} \beta \rho \cos m \omega$ being taken in for the purpose just mentioned, we are left with $\mathrm{F}_{1}$ instead of $\chi$ in (65). Since $\nabla^{4} \mathrm{~F}_{1}=0$, these equations now define a combination of deformations of the persistent or permanent type, under no body force and no surface traction.

The solution therefore resolves itself into

(i) this free deformation of the permanent mode;

(ii) a local perturbation;

(iii) a particular solution giving the proper discontinuity of stress corresponding to the applied areal force. 
The particular solution is

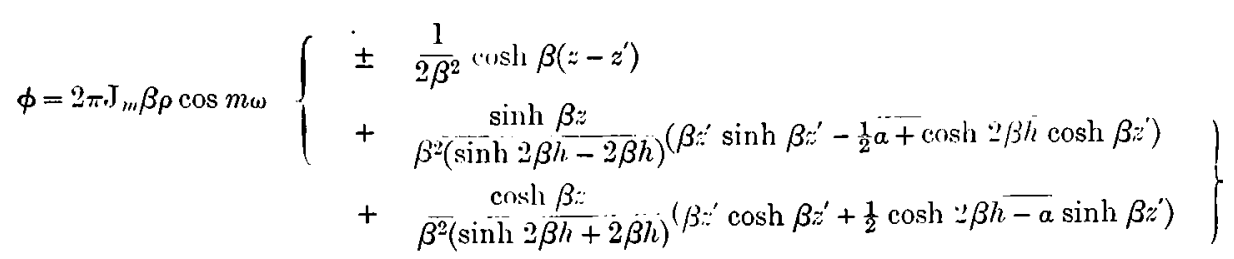

with a corresponding expression for $\theta$, obtainable from (61) by changing $\kappa$ into $\beta$ and then replacing $J_{0} \beta \mathrm{R}$ by $\frac{1}{\beta} 2 \pi \mathrm{J}_{m} \beta \rho \cos m \omega$.

It is easy to verify that this is actually a particular solution. Consider in the first place the analogous forms of $\phi, \theta$ in (61), and for greater generality, suppose $\mathrm{J}_{0} \kappa \mathrm{R}$ replaced by $f(x, y)$ where $\left(\nabla^{2}+\kappa^{2}\right) f=0$.

Then, from the method by which (61) were found, it is obvious that they give no stress across the planes: $= \pm h$. Let us examine the effect of the discontinuity in the forms of $\phi, \theta$ at the plane $z=\varepsilon^{\prime}$, on the displacements and stresses as given in (4), (5).

If we take simply

$$
\begin{aligned}
& \phi=\frac{1}{\partial_{\kappa}} \cosh \kappa\left(z-z^{\prime}\right) f \\
& \theta=-a \cosh \kappa\left(z-z^{\prime}\right) f-z^{\prime} \sinh \kappa\left(z-z^{\prime}\right) f
\end{aligned}
$$

then we find at $z=i^{\prime}$,

$$
\begin{aligned}
& u=v=w=0
\end{aligned}
$$

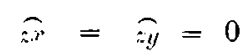

$$
\begin{aligned}
& \widehat{\approx \approx} \quad=-\mu(a+1) \kappa i \text {. }
\end{aligned}
$$

Thus with the complete expression (61), the displacements are continuous, as also the stresses $\approx \approx ; 0$, but $\approx z\left(z=z^{\prime}+\right)$ exceeds $\widehat{z z}\left(z=z^{\prime}-\right)$ by $-2 \mu(\alpha+1) \kappa f$. We thus see that in (70) the corresponding discontinuity in $\approx=$ will be $-4 \pi \mu(\alpha+1) \cdot \mathrm{J}_{m} \beta \rho \cos m \omega$. This continuity of displacement, and discontinuity in $\cdots$, are precisely as demanded by the conditions of equilibrium of the plate.

If we take (61) with $\mathrm{J}_{0} \mathrm{R}$ unaltered, prepare them for integration as in $\$ 19$, multiply by $e^{-\kappa \epsilon}$ and integrate with respect to $\kappa$ from 0 to $\infty$, the discontinuity in $z z$ at $z=z^{\prime}$ will become

$$
-2 \mu(\alpha+1) \int_{11}^{\infty}, \cdots \kappa \epsilon \kappa J_{0} \kappa R d \kappa .
$$

If further we multiply this by $Z\left(x^{\prime}, y^{\prime},:^{\prime}\right) d l_{x} x^{\prime} d y^{\prime}$ and integrate with respect to $x^{\prime}, y^{\prime}$, and then take the limit for $\varepsilon=0$, the discontinuity becomes, in virtue of (11),

$$
-4 \pi \mu(a+1) Z\left(x, y,:^{\prime}\right) \text {. }
$$

We have thus a proof of the solution for an areal distribution of $Z$ force, independent of the infinite solid solution (6), which might itself be found from the beginning by this method. 
20. The general problem of internal normal fince. Alpwrimete forms $\because f^{\prime}$ displacements and stresses.s.

The developmentis uriven in $\$ \$ 14,15$ may olwionsly be applied in the present case also.

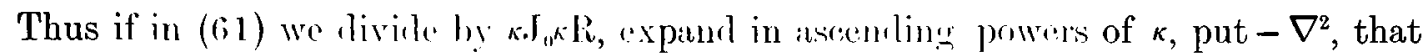

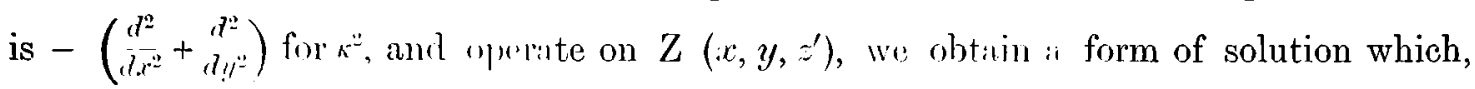
with the unterpretition of $\nabla^{-1 \%}$ and $\nabla^{-2} Z$ given in (38), is simply the foregoing general solution for areal force of intensity $2 \mu(\alpha+1) Z$, armugel in toms of ascending order in $h^{2}$. The solution in this form firils if at $(x, y, z), \%$ or any of its successive derivatives hecome discontinuous, but it has leen shown in $\$ 1+$ how the local perturbation in the neighbourhood of any surfice of discontinuity may be calculatexl.

For the case when $Z$ vanishes outside an area $A$, the principal part of the perturbation at the edge of $A$, when $h$ is small, is found by substituting for $G_{0} k R$ in (63), (64),

$$
-\frac{1}{\kappa^{2}} \int\left\{\mathrm{Z}_{\left(x^{\prime}, y^{\prime}\right)} \frac{d}{d n} \mathrm{G}_{0} \kappa \mathrm{R}-\left(i_{1,}(\kappa \mathrm{R}) \frac{d}{d n} \mathrm{Z}_{\left(x^{\prime}, i^{\prime}\right)}\right\}, l_{*},\right.
$$

where differentiations and integrations have reference to the acceuted coordinates.

Since the solution for the cise when there are any finite number of surfaces at which $\mathrm{Z}$ or its derivatives become disiontinuous an be found from this elementary case by simple summation, we see that discontinuity in the force itself gives rise to values of $\phi, \theta$ in the perturbation terms of order $h^{2}$ at the surface, discontinuity in $\frac{1 Z}{d m}$ to terms of order $h^{3}$ if $Z$ itself is continuous. The next term is of order $h^{4}$ and depends on discontinuity of $\nabla^{2} Z$, that is, of the second derivatives of $Z$, and $s$ on.

The symbolical solution for $Z$ force distributed on the plane $z=z^{\prime}$ with intensity $2 \mu(a+1) Z\left(x, y, z^{\prime}\right)$ per unit aros at $\left(x, y, z^{\prime}\right)$ is given by

$$
\begin{aligned}
& \phi= \pm \frac{1}{2 \kappa^{2}} \cosh \kappa\left(\because-y^{\prime}\right)
\end{aligned}
$$

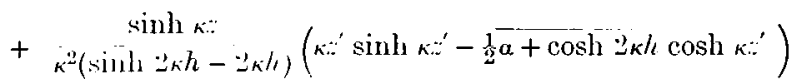

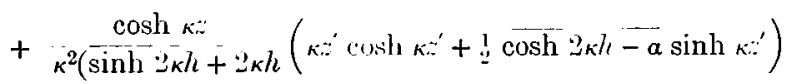

$$
\begin{aligned}
& \theta=\mp \frac{a}{2 \kappa^{2}} \cosh \kappa\left(:-\ddots^{\prime}\right) \mp \frac{\hat{a}^{\prime}}{\kappa} \sinh \kappa\left(\because \because-y^{\prime}\right)
\end{aligned}
$$

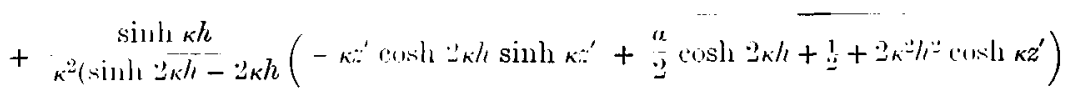

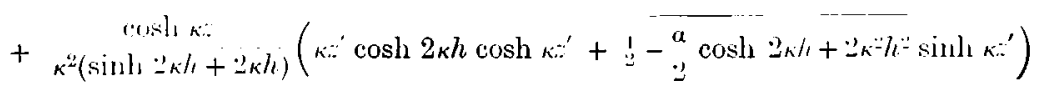

$$
\begin{aligned}
& \text { with } \kappa^{2}=-\nabla^{\prime \prime} \text {, operating on } Z\left(r, y, .^{\prime}\right) \text {. . . . . ( } 71
\end{aligned}
$$

The ipproximate solution is olitained by expanding in ascending powers of $\kappa^{2}$. By retaining only the terms of negative degree in $\kappa^{2}$. each of the displacements will be 
given to a second approximation, and each of the stresses, except $\overline{z z}$, to a first approximation. The result is obviously the same as that found by integrating the permanent terms $(65)$ of the original source solution.

If we write

$$
\mathrm{F}=\iint \mathrm{Z}\left(x^{\prime}, y^{\prime}, z^{\prime}\right) \chi(\mathrm{R}) d x^{\prime} d y^{\prime},
$$

then the displacements for $Z$ force of intensity $Z\left(x, y, z^{\prime}\right)$ per unit area on $z=z^{\prime}$ are

$$
\begin{aligned}
& u=\frac{d}{d x} \frac{3}{32 \pi \mu h^{3}}\left\{-(a+1) z \mathrm{~F}+\Gamma^{2} \mathrm{~F}\left(\frac{a+5}{6} z^{3}+\frac{a-3}{2} z^{\prime 2} z-\frac{+1}{5} h^{2} z+\frac{a-3}{3} h^{2} z^{\prime}\right)\right\} \\
& v=\frac{d}{d y} \\
& w=\frac{3}{32 \pi \mu h^{3}}\left\{(a+1) \mathrm{F}+\nabla^{2} \mathrm{~F}\left(\frac{3-a}{2} z^{2}+\frac{3-a}{2} z^{\prime 2}-4 h^{2}+\frac{a+1}{5} h^{2}\right)\right\} .
\end{aligned}
$$

The corresponding results for a volume distribution of force, $\mathrm{Z}(x, y, z)$ per unit volume, are found by integrating these with respect to $z^{\prime}$ from $-h$ to $+h$.

In order to calculate the stress.$-:$ from displacements, we should need the value of $w$ to a third approximation. It is therefore easier to find $\widehat{z} z$ directly from (71) and (5). On dividing by $2 \mu(\alpha+1)$, we find, corresponding to (7:),

$$
\hat{\approx}=\mp \frac{1}{2} Z+\frac{3 h^{2}-z^{3}}{4 h^{3}} \mathrm{Z}
$$

When the force is $Z(x, y, z)$ per unit volume, this leads to

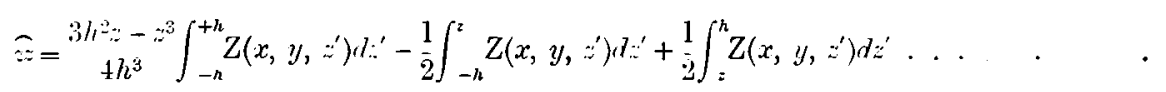

We can now find the stresse: $\widehat{x} \vec{x}, \widehat{x} !$, , Iy to a second approximation.

For

whence, eliminating $d w$,

$$
\begin{aligned}
& \hat{x}=(\lambda+2 \mu) \frac{d \prime \prime}{d x}+\lambda \frac{d l^{\prime \prime}}{d ! j}+\lambda_{d !}^{\prime \prime \prime \prime} \\
& \widehat{\approx}=\lambda \frac{d u}{d x}+\lambda \frac{d v}{d y}+(\lambda+2 \mu)_{i z:}^{d x v},
\end{aligned}
$$

and similarly

$$
\widehat{x x}=\frac{4 \mu(\lambda+\mu)}{\lambda+2 \mu}\left(\frac{d u}{d x}+\sigma \frac{d v}{d y}\right)+\frac{\lambda}{\lambda+2 \mu} \widetilde{z x}
$$

Also

$$
\widehat{y y}=\frac{4 \mu(\lambda+\mu)}{\lambda+2 \mu}\left(\sigma \frac{d u}{d x}+\frac{d v}{d y}\right)+\frac{\lambda}{\lambda+2 \mu}-\widehat{z z}, \text { where } \quad \sigma=\frac{1}{2} \lambda /(\lambda+\mu) .
$$

$$
\widehat{x y}=\mu\left(\frac{d u}{d y}+\frac{d v}{d x}\right)
$$

We have now only to put in the values of $u, v$ from (72) and the value of $\widehat{z z}$ from (73). 
If we denote $3 \begin{aligned} & 3 \\ & 32 \pi \mu h^{3}\end{aligned}(\alpha+1) \mathrm{F}$, the principal term in $*$ in $(7 \%)$, by $\mathrm{W}$, we have

or

$$
\begin{aligned}
\nabla^{4} \mathrm{~W} & =\frac{3}{32 \pi \mu h^{3}}(a+1) \nabla^{4} \mathrm{~F} \\
& =\frac{\vdots}{16 \mu h^{3}} \cdot(a+1) \mathrm{Z} \\
& =\frac{3}{8 \mu h^{3}} \frac{\lambda+2 \mu}{\lambda+\mu} \mathrm{Z} \\
\mathrm{C} \nabla^{4} \mathrm{~W} & =Z, \text { where } \quad \because=\frac{8}{3} \mu h^{3}(\lambda+\mu /(\lambda+2 \mu)
\end{aligned}
$$

In this notation, to a first approximation

Again from (72),

$$
\begin{aligned}
& \hat{c x}=-\frac{3 c^{2}}{2 h^{3}}\left(\begin{array}{l}
d^{2} \mathrm{~W} \\
d x^{2}
\end{array}+\sigma_{d y^{2}}^{d \prime W}\right) \\
& \widehat{y y}=-\frac{3 \mathrm{C}}{2 h^{3}} \cdot\left(\sigma^{\prime 2} d x^{2}+\frac{d^{2} \mathrm{~W}}{d y^{2}}\right) \\
& x_{i !}=-\frac{3 \mathrm{C}}{2 h^{3}}(1-\sigma) d \frac{d^{2} \mathrm{~W}}{d x} d y .
\end{aligned}
$$

$$
\begin{aligned}
\widehat{\approx x} & =\mu\left(\frac{d u}{d z}+\frac{d w}{d x}\right)=\frac{3 \mu}{32 \pi \mu h^{3}} \cdot 4\left(z^{2}-h^{2}\right) \frac{d}{d x} \nabla^{2} \mathbf{F} \\
& =\frac{3}{4 h^{3}} \mathrm{C}\left(z^{2}-h^{2}\right) \frac{d}{d x} \nabla^{2} \mathrm{~W}
\end{aligned}
$$

23. Normal force a function of $\mathrm{z}$ only.

It may be useful to put down here the next term in the development of $w$, of which the two principal terms are given in (72). This is

$$
\begin{gathered}
\frac{3}{32 \pi \mu h^{3}} \nabla^{4} \mathrm{~F}\left\{\begin{array}{c}
a-7 \\
24\left(z^{4}+z^{\prime 4}\right)+\frac{(a-3)^{\prime \prime}}{4(a+1)}: z^{\prime 2}+\left(\frac{3-a}{10}+\frac{2 a-2}{a+1}\right) h^{\prime 2}\left(z^{2}+z^{\prime 2}\right)+\left(\frac{4}{3 a+3}-\frac{4}{5}+\frac{11 a+11}{3 \cdot 5^{2} \cdot 7}\right) h^{4} \\
+\frac{(3-a)^{2}}{3 a+3} h^{2} z z^{\prime}
\end{array}\right\} . \\
\quad \pm(1-a)\left(z-z^{\prime}\right) Z\left(x, y, z^{\prime}\right) .
\end{gathered}
$$

In this, of course,

$$
\nabla^{4} \mathrm{~F}=2 \pi Z\left(x, y, z^{\prime}\right)
$$

The terms which have to be added to $(72),(78)$ in order to give the complete particular values of $u, v, w$, all contain $x, y$ derivatives of $\nabla^{4} \mathrm{~F}$ or $\mathrm{Z}$. Hence, if $\mathrm{Z}\left(x, y, z^{\prime}\right)$ is a function of $z^{\prime}$ alone, (72) and (78) give a complete particular solution of the problem. Further, $\%$ may have one constant value in one region of the plane $z=z^{\prime}$, and another constant value in another region of that plane. (72), (78) will still give a particular solution in each of those regions taken separately, or rather in the cylindrical spaces of which these regions are sections, but it ought to be carefully noticed that it is not in general an exact solution when the two regions are considered together as part of one body. The point of failure is, it need scarcely be said, the condition of synexis ; 
the two particular solutions do not fit, that is, they do not give the same values for displacements and strains on the two sides of the cylindrical surface or surfaces of discontinuity.

On the other hand, the supplementary terms required in order to make the solution synectic belong to what we have (alled the decaying type. They give rise to displacements and strains of infinitely high order, if we may so speak, in the small quantity $h$, except very near the surfaces of discontinuity. This being so, we need not be surprised to find that the solution (72), (78) is not necessarily the simplest particular solution in any one region within which $Z$ is continuously constant.

Thus, for example, if we pick out the terms which contain $z^{\prime 2}$ as a factor, we find displacements proportional to

$$
\begin{aligned}
& u=\frac{d}{d x}:-i \nabla^{2} \mathrm{~F} \\
& v=\frac{d}{d ! y}, \\
& m=\nabla^{2} \mathrm{~F}+\nabla^{4} \mathrm{~F}\left(\frac{3-a}{a+1} \frac{1}{2^{2}} z^{2}+\mathrm{C}_{1} h^{2}\right)
\end{aligned}
$$

which belong to the type ( 23 ), and contribute nothing to body force or face tractions. These terms might therefore be omitted in any problem where the condition of synexis is irrelevant, and in particular when the object is merely to obtain a particular solution for body force and face traction in a problem relating to a finite solid.

\section{Internal firce parallel to the froces.}

We will now go on to consider the problem of force applied to the body in a direction parallel to the faces of the plate.

A force of $4 \pi \mu(a+1)$ units applied at $\left(x^{\prime}, y^{\prime}, z^{\prime}\right)$ in the direction of $a x$ gives in an infinite solid displacements defined, according to (9), by

$$
\begin{aligned}
& \psi=-\frac{n+1}{2} \frac{d}{d ! ! \prime} d d^{2-u+1} \cdot 2 \\
& \left.\theta=-\begin{array}{ccc}
a & \frac{d}{d x} & d^{-3} r^{-1} \\
2 & d z^{-2} & +z^{\prime} \frac{d}{d s} \frac{d^{-1} r^{-1}}{d z^{-1}}
\end{array}\right\} \\
& \phi=-\frac{1}{2} \frac{d}{d x} \frac{d d^{-2 p^{-1}}}{d z^{-2}}
\end{aligned}
$$

Honce the tractions which such a force produces on $z= \pm h$ will be neutralised by a system $\psi, \theta, \phi$ for which

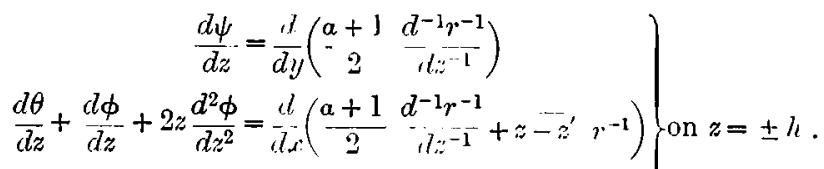

$$
\begin{aligned}
& \left.\frac{d^{2} \theta}{d z^{2}}-\frac{d l^{2} \phi}{d z^{2}}+2 z \frac{d^{3} \phi}{d z^{3}}=\frac{d}{d x}\left(\frac{a-1}{z} r^{-1}+\overline{z-z^{\prime}} \frac{d r^{-1}}{d z}\right)\right)
\end{aligned}
$$


These conditions ane satisfiesl if

and

$$
\psi=\frac{d \psi^{\prime}}{d ! y}, \theta=\frac{d \theta^{\prime}}{d l r^{\prime}}, \phi=\frac{d \phi^{\prime}}{d / x} .
$$

We may take

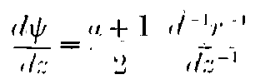

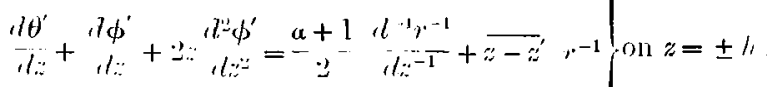

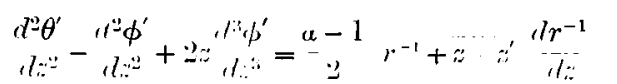

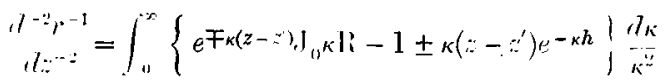

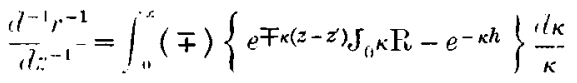

$$
\begin{aligned}
& r^{-1}=\int_{0}^{x} \mp \kappa(z-z), J_{1} \kappa R, R
\end{aligned}
$$

upper or lower signs being taken, as all along, according as $\dot{z}>z^{\prime}$ or $z<z^{\prime}$. We therefore determine provisional values of $\psi^{\prime}, \theta^{\prime}, \phi^{\prime}$, such that

$$
\begin{aligned}
& \frac{1 \psi^{\prime}}{1 /:}=\mp \frac{a+1}{2 \kappa} e^{\mp \kappa\left(z-z^{\prime}\right) J_{0} \kappa} \mathrm{R} \\
& \left.\frac{d \theta^{\prime}}{d z}+\frac{d \phi^{\prime}}{d z}+2 z \frac{d^{2} \phi^{\prime}}{d z^{2}}=\left(\mp \frac{a+1}{3_{K}}+z-z^{\prime}\right) e^{\mp \times(z-z) \mathrm{J}_{0} \kappa} \mathrm{R}\right\} \text { on } z= \pm h \\
& d^{2} \theta^{\prime}-\frac{d^{2} \phi^{\prime}}{d z^{2}}+2: \frac{d^{3} \phi^{\prime}}{d z^{3}}=\left(\frac{\alpha-1}{2} \mp \kappa \overline{-z^{\prime}}\right) e^{\mp \kappa(2-z) \mathrm{J}_{0^{k}} \mathrm{R}}
\end{aligned}
$$

These provisional values are easily found to be

$$
\begin{aligned}
& \psi^{\prime}=\frac{a+1}{2 \kappa^{2}} \cosh \kappa\left(z-z^{\prime}\right) \\
& \left.+\frac{a+1}{2 \kappa^{2} \cosh \kappa h} \sinh \kappa / \sinh \kappa z^{\prime} \sinh \kappa z\right\} \mathrm{J}_{10} \kappa \mathrm{R}
\end{aligned}
$$

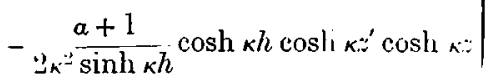

$$
\begin{aligned}
& \phi^{\prime}={ }_{2 \kappa^{\prime 2}}^{1} \cosh \kappa\left(z-z^{\prime}\right)
\end{aligned}
$$

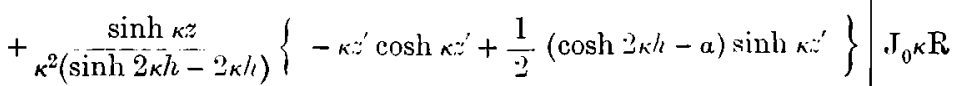

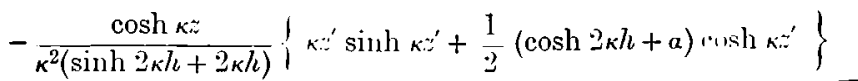

$$
\begin{aligned}
& \theta^{\prime}=\frac{a}{2 \kappa^{2}} \cosh \kappa\left(z-z^{\prime}\right)-\frac{\ddot{z}^{\prime}}{\kappa} \sinh \kappa\left(\ddot{\left.z-z^{\prime}\right)}\right.
\end{aligned}
$$

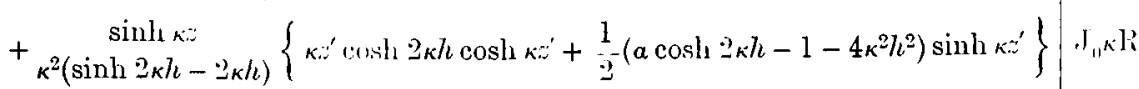

$$
\begin{aligned}
& -\frac{\cosh \kappa z}{\kappa^{2}(\sinh 2 \kappa h+2 \kappa h)}\left\{\kappa z^{\prime} \cosh 2 \alpha h \sinh \kappa z^{\prime}+\frac{1}{2}\left(\alpha \cosh 2 \kappa h+1+4 \kappa^{\prime \prime} l z^{\prime \prime}\right) \cosh \kappa z^{\prime}\right\} \mid
\end{aligned}
$$

TRA.N. ROY. SOC. EDIN., VOI. XLI. PART I. (NO. 8). 
The source itself is similarly given by the temporary values

$$
\left.\begin{array}{l}
\psi^{\prime}=-\frac{a+1}{2 \kappa^{2}} \\
\theta^{\prime}=-\frac{a}{2 \kappa^{2}} \mp \frac{z^{\prime}}{\kappa} \\
\phi^{\prime}=-\frac{1}{2 \kappa^{2}}
\end{array}\right\} \epsilon^{\mp \kappa\left(z-z^{\prime}\right) \mathrm{J}_{0} \kappa \mathrm{R}}
$$

Thus, when the source is included, the provisional values of $\psi^{\prime}, \theta^{\prime}, \phi^{\prime}$ are as in (80), but with the first lines altered,

$$
\begin{aligned}
& \text { in } \psi^{\prime} \text { to } \pm \frac{a+1}{2 \kappa^{2}} \sinh \kappa\left(z-z^{\prime}\right) \\
& , \phi^{\prime}, \pm \frac{1}{2 \kappa^{2}} \sinh \kappa\left(z-z^{\prime}\right) \\
& , \theta^{\prime}, \pm \frac{a}{2 \kappa^{2}} \sinh \kappa\left(z-z^{\prime \prime}\right) \mp \frac{z^{\prime}}{\kappa} \cosh \kappa\left(z-z^{\prime}\right)
\end{aligned} \mid J_{0} \kappa \mathrm{R}
$$

25. Solution of the problem of internal force parallel to the faces.

From these expressions the solution in the form of definite integrals, and finally of series, is obtained as in the previous cases. After the explanations already given, it will be sufficient to write down the final results. For the transitory part of the solution,

$$
\begin{aligned}
& \psi^{\prime}=\sum_{\kappa}(a+1) \frac{1}{\kappa^{2} / 1} \sinh \kappa z^{\prime} \sinh \kappa z \mathrm{G}_{0} \kappa \mathrm{R},(\kappa \text { a pos.-imag. root of } \cosh \kappa h) \\
& -\sum_{\kappa}(a+1)_{\kappa^{i=h}}^{1} \cosh \kappa \kappa^{\prime} \cosh \kappa * \mathrm{G}_{0} \kappa \mathrm{R},(\kappa \text { a pos. imag. root of } \sinh \kappa h) \text {. }
\end{aligned}
$$

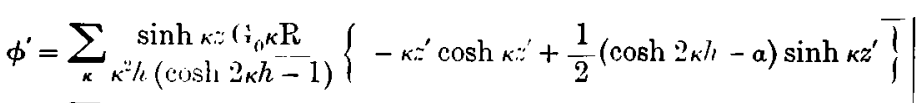

$$
\begin{aligned}
& \theta^{\prime}=\sum_{\kappa} \text { same as previous line multiplied by }(-\cosh 2 \kappa h)
\end{aligned}
$$

where $\kappa$ is a zero of $\sinh 2 \kappa h-2 \kappa h$, with pos. imag. part.

With

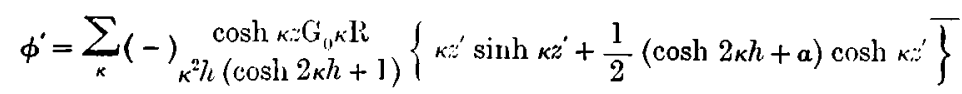

$$
\begin{aligned}
& \theta^{\prime}=\sum_{\kappa} \text { same as previous line multiplied by } \cosh 2 \kappa \lambda^{\prime} \text {. }
\end{aligned}
$$

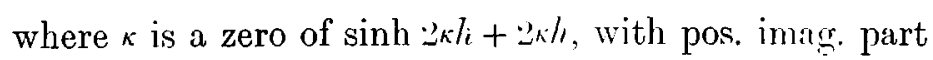

We may recall the method of obtaining the permanent terms. Taking any one of the functions of (80), altered as in (81), we omit the factor $J_{11} R$, and then find its expansion near $\kappa=0$ to contain terms of negative degree in $\kappa$, say $A / \kappa^{3}+B / \kappa$. The 
permanent part of this function is then $A \chi(R)-P^{2} \nabla^{2} \chi(R)$. We thus find for this part of the solution

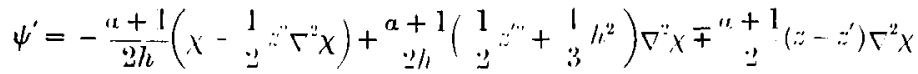

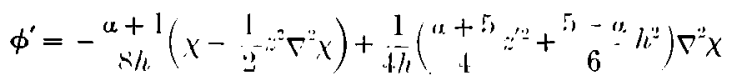

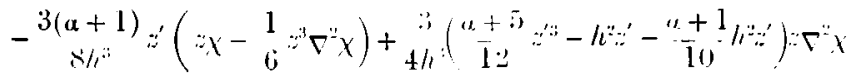

$$
\begin{aligned}
& \mp \frac{1}{\because i}\left(\because-a^{\prime}\right) \Gamma^{\prime \prime} x \\
& \theta^{\prime}=-\frac{a+1}{s / h}\left(x-\frac{1}{2} r^{2} x\right)+\frac{1}{4 h}\left(\frac{a+5}{4} z^{2}+\frac{5-a}{6} h^{2}+a+1 h^{2}\right) \Gamma x
\end{aligned}
$$

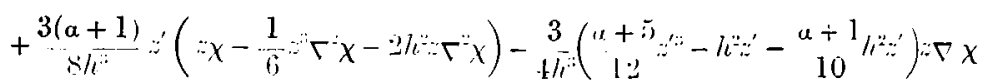

$$
\begin{aligned}
& \mp \frac{a}{2}\left(z-z^{\prime}\right) \Gamma^{\prime \prime} x \pm z^{\prime} \Gamma^{\prime \prime} x
\end{aligned}
$$

When $z^{\prime}$ is put equal to $h$ in the above values of $\psi^{\prime}, \phi^{\prime}, \theta^{\prime}$ it will be found, with very little trouble, that they reduce to those of $(-18) \ldots .(51)$, multiplied by $\frac{1}{2}(\alpha+1)$. (Cf. $\$ 20$.) As in $\$ \geq 0$, the displacements due to the ambiguous terms in (83) are null if $R>0$. But there is this difference in the present case, that they do not continue to vanish in the corresponding solution for an areal distribution of force on $z=z^{\prime}$.

If the intensity of the distribution is $\mathrm{X}\left(x, y, v^{\prime}\right)$ per unit area at $(x, y)$, this solution is defined as in (79), $\psi^{\prime}, \theta^{\prime}, \phi^{\prime}$ being obtained from (82), (83) by muitiplying by

$$
\frac{1}{4 \pi \mu(a+1)} \mathrm{X}\left(x^{\prime}, y^{\prime}, z^{\prime}\right) \pi x^{\prime}, 1 y^{\prime}
$$

and interrating over the area within which $\mathrm{X}$ is tinite.

When this is done we find that the ambiguous terms lead to

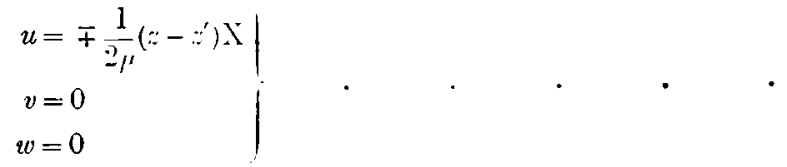

In verification, we observe that these displacements are continuous above and below the plane $z=z^{\prime}$, and that the corresponding stresses are also continuous with the exception of $z$, the value of which just below $z=z^{\prime}$ exceeds its vilue just above by $\mathrm{X}$. The value of $z$ : being $\mp \frac{1}{2} \mathrm{X}$, we have for the contribution of $(84)$ to the resultant $\int_{-h}^{+h} \overrightarrow{z x} d z$,

$$
\frac{1}{2} x\left(\int_{-h}^{\prime} d z-\int^{n} d z\right)=: x
$$


20. Alproximate values of the displacements. Lagrange's equation for flexure to a second approximation.

The unambiguous terms in (83), as in (82), fall naturally into two classes, in the first of which $\psi^{\prime}, \theta^{\prime}, \phi^{\prime}$ are odd functions of $z$, while in the second they are even. Of the displacements derived from the first class, $u$ and $v$ are odd, and $v$ even in $z$, and the strain may be described as flexural. In the other class $u, "$ are even, and $w$ odd in $z$, and the strain may be described as extensional. A force $\mathrm{X}$ at $\left(x^{\prime}, y^{\prime}, z^{\prime}\right)$ acting along with a parallel but oppositely directed force $\mathrm{X}$ at $\left(x^{\prime}, y^{\prime},-z^{\prime}\right)$ would give rise to flexural strain only; equal and similarly directed $X$ forces at these two points to extensional strain only. This follows at once from the fact that the terms of $\psi^{\prime}, \theta^{\prime}, \phi^{\prime}$, which are odd in $z$, are also odd in $z^{\prime}$, and vice verse.

The distribution of force being $\mathrm{X}\left(x, y, z^{\prime}\right)$ per unit area at $(x, y)$ on $z=z^{\prime}$, let

$$
\mathrm{F}=\frac{l l}{d x} \iint \mathrm{X}\left(x^{\prime}, y^{\prime}, z^{\prime}\right) \chi(\mathrm{R}) d x^{\prime} d y^{\prime}
$$

Then from the flexural part of (83),

$$
\begin{aligned}
& \left.\begin{array}{l}
\phi=-\frac{3}{32 \pi \mu l l^{3}}:\left(\because \mathrm{F}-\frac{1}{6} z^{3} \nabla^{2} \mathrm{~F}\right) \\
\theta=\frac{3}{32 \pi \mu l^{3}} \because\left(\because \mathrm{H}-\frac{1}{6} z^{3} \nabla^{2} \mathrm{~F}-2 h^{2} z \nabla^{2} \mathrm{~F}\right)
\end{array}\right\} \text { with } \\
& \left.\begin{array}{l}
\phi=-\nabla^{\prime \prime F} \\
\theta=\nabla^{2} \mathrm{~F}
\end{array}\right\} \text { each multiplied by } \frac{3}{32 \pi \mu h^{3}} \cdot \frac{2}{a+1}\left(\frac{a+11}{10} h^{2} \alpha^{\prime}-\frac{a+5}{12^{-}} z^{\prime 3}\right)
\end{aligned}
$$

These lead to

$$
\begin{aligned}
& \begin{array}{l}
u=\frac{d}{d x} \mid \underset{3}{3} \\
v=\frac{d}{d y} \mid 32 \pi \mu h^{3}
\end{array}\left\{-(a+1) z^{\prime} \mathrm{F}+\nabla^{2} \mathrm{~F}\left(\frac{a+5}{6} z^{\prime} z^{3}+\frac{a+5}{6} z^{\prime 3 z z}-\frac{a+21}{5} h^{2} z^{\prime} z\right)\right\} \\
& w=\frac{3}{32 \pi \mu h^{3}}\left\{(a+1) \mathrm{F}+\Gamma^{2} \mathrm{~F}\left(\frac{3-a}{2} z^{2}+\frac{a+1}{5} h^{2} z^{\prime}-a+5 ; y^{\prime 3}\right)\right\} . \quad .
\end{aligned}
$$

For $\mathrm{Y}$ force the same expressions hold if we take $\mathrm{F}=\frac{1}{d y} \iint \mathrm{Y}\left(x^{\prime}, y^{\prime}, x^{\prime}\right) \chi(\mathrm{R}) d x^{\prime} d y^{\prime}$.

These formulæ, with all of (72) but the last terms of $u, v$, and with the odd parts in $\approx$ arising from the ambiguous terms, give to a second approximation the displacements of the flexural mode under any forces. The differential equation sitisfied by $\bar{u}$, the normal displacement of the mirl plane, or value of $w$, for $\approx$ equal to zero, is important in the history of the approximate theory. We can lww write it down to a second approximation, namely, with $\mathrm{C}$ is in (75),

$$
\begin{aligned}
& \mathrm{CV}^{4} \bar{w}=\mathrm{Z}+z^{\prime}\left(\frac{d \mathrm{X}}{d j}+\frac{d \mathrm{Y}}{d y}\right)
\end{aligned}
$$

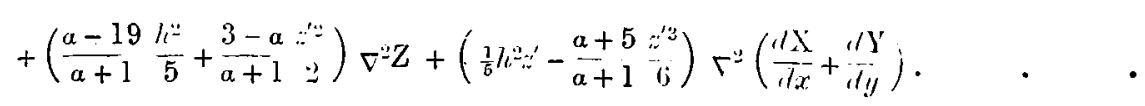


This equation wives the result for an areal distribution $X, Y, Z$ on $z=z^{\prime}$. For traction on $z= \pm h$ replace $z^{\prime}$ ly $\pm h$; for a volume rlistrilution $\mathrm{X}, \mathrm{Y}, \mathrm{\gamma}$ replace

and so on.

$$
\begin{aligned}
& Z \text { by } \int_{-h}^{h} Z\left(x, y, z^{\prime}\right) d z^{\prime}
\end{aligned}
$$

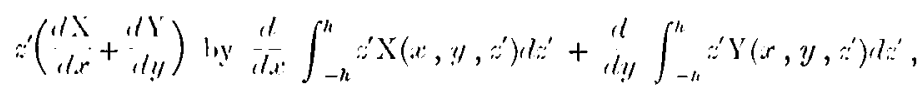

27. Extrusional strain. Differential equations of the prineipal mode.

T'he unambigums extensional terms of (83) remain to be considered.

Write $\mathrm{E}=\frac{1}{3 z^{2} \pi \mu h} \iint \mathrm{X}\left(x^{\prime}, y^{\prime}, z^{\prime}\right) \chi(\mathrm{R}) \cdot d x^{\prime} d y^{\prime}$.

Then for an areal distribution $\mathrm{X}$, these terms are

$$
\begin{aligned}
& \psi^{\prime}=-4\left(\mathrm{E}-\frac{1}{2}:^{\prime \prime} \nabla^{2} \mathrm{E}\right)+4\left(\frac{1}{2} z^{\prime \prime \prime}+\frac{1}{3} h^{2}\right) \nabla^{2} \mathrm{E} \\
& \theta^{\prime}=-\left(\mathrm{E}-\frac{1}{2} \nabla^{\prime \prime} \mathrm{E}\right)+\frac{2}{a+1}\left(\frac{a+5}{4}:^{\prime \prime 2}+\frac{5-a}{6}-h^{2}+a+1 h^{2}\right) \nabla^{2} \mathrm{E} \\
& \phi^{\prime}=-\left(\mathrm{E}-\frac{1}{2}: \nabla^{*} \mathrm{E}\right)+\frac{2}{a+1}\left(\begin{array}{c}
a+5 \\
4
\end{array}\right.
\end{aligned}
$$

The second parts of these expressions give

$$
\begin{aligned}
& u=(a-3)\left(\frac{1}{2} z^{\prime \prime}-\frac{1}{3} h^{2}\right) \frac{d^{\prime \prime}}{d r^{2}} \nabla^{2} \mathrm{E}+4\left(z^{\prime \prime}+\frac{2}{3} h^{2}\right) \nabla^{\prime \prime} \\
& v=(\alpha-3)\left(\frac{1}{2} z^{\prime 2}-\frac{1}{3} h^{2}\right) \frac{d^{2}}{d x d y} \nabla^{2} \mathrm{E} \\
& w=0
\end{aligned}
$$

The first parts give

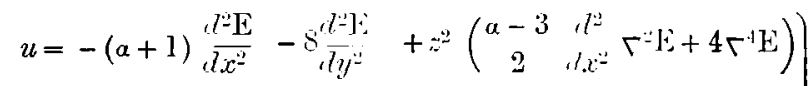

$$
\begin{aligned}
& v=-(a+1) \frac{d l^{2} \mathrm{E}}{d x d y}+8 \frac{d^{2} \mathrm{E} !}{d x d !}+\because^{2} \quad \frac{a-3}{2} \frac{d^{2}}{d x d !} \Gamma^{2} \mathrm{E} \\
& w=(3-a)=\frac{d}{1}+\frac{1}{x} \Gamma^{*}
\end{aligned}
$$

If now further we write $\quad \mathrm{K}=\frac{1}{32 \pi \mu h} \iint \mathrm{Y}\left(x^{\prime}, y^{\prime}, z^{\prime}\right) X(\mathrm{R}), l x^{\prime}, l y^{\prime}$

the corresponding displacements for a distribution of $\mathrm{Y}$ force on $z=z^{\prime}$ can at once be written down from symmetry. The results for $\mathrm{X}$ and $\mathrm{I}$ force combined cannot conveniently be expressed in terms of one function, as in the case of the flexural mode, and the best plan is probably to put everything in terms of the principal values of $u, v$, namely,

$$
\begin{aligned}
& \mathrm{U}=-(a+1) \frac{d^{2} \mathrm{E}}{d x^{2}}-8 \frac{d^{2} \mathrm{E}}{d y^{2}}-(a+1) \frac{d^{2} \mathrm{~K}}{d x}+8 \frac{d^{2} \mathrm{k}}{d x}+ \\
& \mathrm{V}=-(a+1) \frac{d^{2} \mathrm{E}}{d x+l y}+8 \frac{d^{2} \mathrm{E}}{d x \cdot d ! y}-(a+1)^{\prime r^{2} \mathrm{~K}} \frac{d^{2}}{d y^{2}}-8 \frac{d^{2} \mathrm{~K}}{d x^{2}} j
\end{aligned}
$$


We have then

$$
\begin{aligned}
& \left.u=\mathrm{U}+\frac{3-a_{a+1}\left(1, z^{2}+1, y^{\prime \prime}-\frac{1}{3} h^{2}\right)}{d x} \frac{d}{d x}\left(\frac{d \mathrm{U}}{d x}+\frac{d \mathrm{~V}}{d y}\right)+\left(1 z^{2}+\frac{1}{2} z^{\prime 2}+\frac{1}{3} h^{2}\right) \frac{\mathrm{X}}{2 \mu h}\right) \\
& v=\mathrm{V}+\frac{3-a}{a+1}\left(\frac{1}{2} z^{2}+\frac{1}{2} y^{2}-\frac{1}{3} h^{2}\right) \frac{d}{d y}\left(\begin{array}{l}
d 1 \\
1
\end{array}\right. \\
& w=\frac{a-3}{a+1}=\left(\frac{l \mathrm{U}}{d t}+\frac{l \mathrm{lV}}{d ! n}\right)
\end{aligned}
$$

The ordinary approximate theory obtains differential equations to determine $\mathrm{U}, \mathrm{V}$. These are easily found by eliminating $\mathrm{E}$ and $\mathrm{K}$ in turn from (88).

Thus

and

$$
\begin{aligned}
& \frac{d \mathrm{U}}{d x}+\frac{d \mathrm{~V}}{d y}=-(a+1)\left({ }_{d x}^{\prime \prime} \Gamma^{2} \mathrm{E}+{ }_{d, y}{ }^{\prime \prime}{ }^{2} \mathrm{~K}\right) \\
& \frac{d l i}{d ! l}-\frac{d \mathrm{~V}}{d x}=\quad-s\left(\frac{d}{d y} \nabla^{2} \mathrm{E}-\frac{d}{d c^{2}} r^{2} \mathrm{~K}\right)
\end{aligned}
$$

$$
\begin{aligned}
& \frac{1}{a+1} \frac{d}{d y}\left(\frac{d \mathrm{U}}{d y}+\frac{d \mathrm{~V}}{d y}\right)+\frac{1}{d} \frac{d}{d y}\left(\frac{d \mathrm{U}}{d ! y}-\frac{d \mathrm{~V}}{d x}\right)=-\frac{\mathrm{X}}{16 \mu h} \\
& \frac{1}{a+1} \frac{d}{d !}\left(\frac{d \mathrm{~V}}{d y}+\frac{d \mathrm{~V}}{d y}\right)-\frac{1}{d y} \frac{d}{d x}\left(\frac{d \mathrm{U}}{d y}-\frac{d \mathrm{~V}}{d x}\right)=-\frac{\mathrm{Y}}{16 \mu h}
\end{aligned}
$$

The principal parts of the contribution of $Z$ force to extensional displacements appear in $(72),(78)$.' In the notation of those formulie

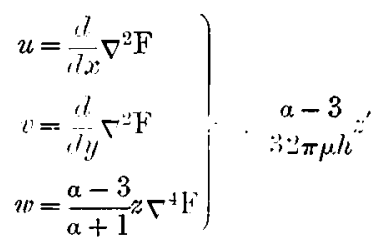

with in addition, $w=$ the odd part in $:$ of the ambiguous term in $(78)$.

If these last values of $u, v$ be included in the principal values $U, V$, then the righthand members of $(90)$ will become respectively

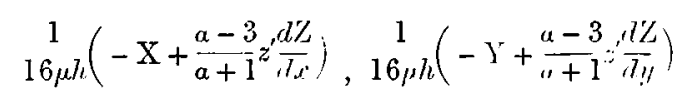

28. Approvimate malues "f the stresses ceross "phome prevellel to the faces.

For any distribution of fores parallel to the faces of the plate, the formula of $\S \S 26,27$ give the terms of the two lowest orders in the values of $u, v$, and the term of lowest order in $\omega^{*}$ From these terms we can calculate all the stresses but $\ldots$ to a first approximation, and as in $\$ 2$, , when the first term of $\widehat{z z}$ is known, we can find

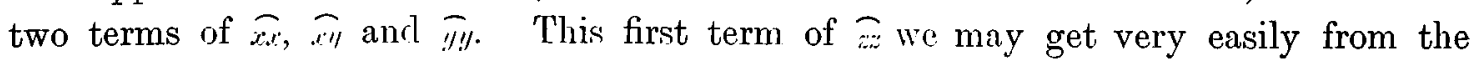
symbolical form of the solution corresponding to $(80),(81)$. Thus for areal force $\mathrm{X}$

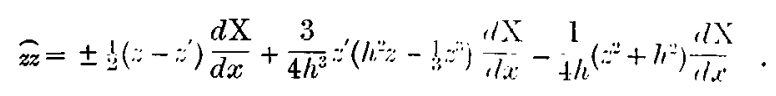

* There should be added from ( 84 ) llwe term.

$$
\left\|=\mp\left(:-\because^{\prime}\right) \mathrm{X} / \mu, \quad v=\mp\left(:-:^{\prime}\right) \mathrm{Y} / 2 \mu, \quad\right\| \stackrel{=}{=} 0 .
$$


From $(84),(86),(89)$ for areal forre $\mathrm{X}$

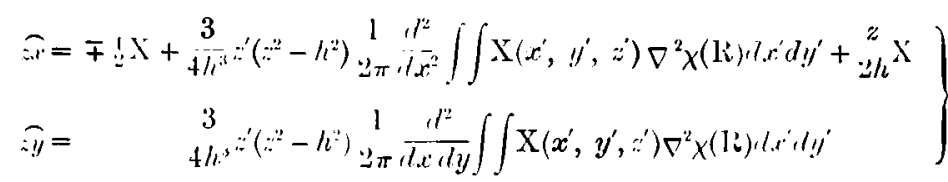

It may be verified that these give zero stress on $z= \pm 1$, and $\frac{d y}{d x}+\frac{d y}{d y}+\frac{d \bar{z}}{d z}=0$.

From the formulie we have given, it is of course merely a matter of the simplest algebra to calculate any of the stresses to whatever criler of approximation may be required, but it may be worth while to remark here that the fundamental equations of equilibrium (1) may be userl with great advantage in obtaining the principal results. If, for example, we know only the first terms of $x, \ldots \%$, I.!. the two first of these equations would give the first terms of $z x, \hat{y}$ by a simple intention with respect to $z$, and then the list equation would give the first term of..- . Similarly, when the first

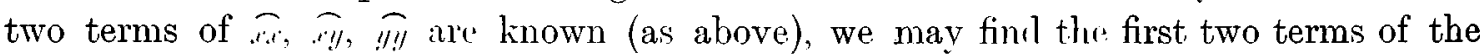
other stresses.

29. Transmission of foren to a listance. Erpronsions in pular coordinates.

We have up to this point been considering mainly the particular solution to which our general source solutions lead for any given distribution of force; or, as we may say, we have been investigating the effect of any given force system on that part of the solid to which the force is applied. But it is also of great interest to inquire what is the effect of this force at points of the solid remote from its region of application. It is obvious that we obtain a sufficient answer to this question by retaining only the permanent terms in the source solutions, those terms, namely. which are given in (65) and (83).

For force applied only at points on a given normal to the plate, these formula are all that we require. They show at a glance that the distant effect depends chiefly on resultant forces and couples, but not entirely, since $z^{\prime}$ and $z^{\prime 2}$ oceur in the formulæ for $\mathrm{Z}$ force, and $z^{\prime \prime}, z^{\prime \prime}$ in those for $\mathrm{X}$ force. When the force is not confined to a line, but is distributed over a finite volume of the solid, the result is obtained in more intelligible form if before integration the function $\chi$ is suitably expanded so as to yield a series of solutions in which accented and unaccented coordinates are explicitly separated. The most nonvenient expansion of $\chi$ is in terms of polar coordinates as given in $(e)$ of the introlustory section.

Suppose, then, a single form applied at the point $\left(. r_{1}, y_{1}, i_{1}\right)$ or $\left(\rho_{1}, n_{1}, z_{1}\right)$, the components of the force heing $\mathrm{X}_{1}, \mathrm{Y}_{1}, \mathrm{Z}_{1}$, parallel to the restingular axes, or $\mathrm{P}_{1}, \Omega_{1}, \mathrm{Z}_{1}$ parallel to radius vector, transverse, aml ixis of $z$. We have to find the displacements at $(\rho, \omega, z)$ where we suppose $\rho>\rho_{1}$.

For an $\mathrm{X}$ force, the value of $\psi$ is $\frac{d \psi^{\prime}}{d y}$ with $\psi^{\prime}$ : pending on the magnitude of $\mathrm{X}$ being for the moment suppressed. 
This is the same as $-\frac{d \psi^{\prime}}{d y}$ or $(-)$ rate of variation of $\psi^{\prime}$ in the direction perpendicular to the force at its point of application.

Similarly $\quad \phi=-\frac{l \phi^{\prime}}{d x^{\prime}}=(-)$ rate of variation of $\phi^{\prime}$ in direction of force.

Hence for

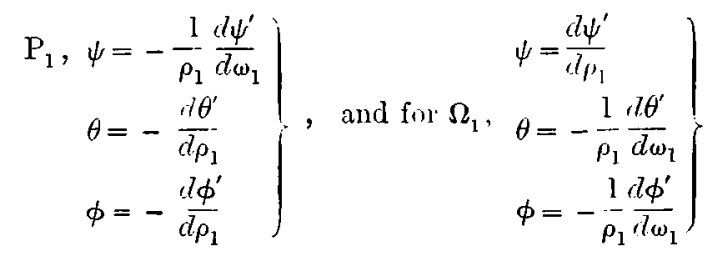

We shall take separately the extensional and flexural parts of the solution.

Also in the following $u, r$ are the displacements along radius vector and tran werse.

I. Extensional terms.

The following solutions occur.

(i) $\left.\begin{array}{rl}u & =\left(\frac{7-a}{4}-\frac{9+a}{2} \log \frac{\rho}{2}+3-a+y^{-2}\right) \cos \omega \\ v & =\left(\frac{7-a}{4}+\frac{9+a}{2} \log \frac{\rho}{2 h}+\frac{3-a}{2} z^{-2}\right) \sin \omega \\ w & =(3-\alpha) z p^{-1} \cos \omega\end{array}\right\}$

(ii) Same as (i) with $\cos \omega$ changed into $\sin \omega$, and $\sin \omega$ into $-\cos \omega$

(iii) When $m>1$,

$$
\begin{aligned}
& u=\left\{\frac{8 m-(m-2)(a+1)}{4(m-1)} \rho^{-m+1}+\frac{3-a}{2}-m \omega^{2} \rho^{-m-1}\right\} \cos m \omega \\
& l=\left\{\therefore\left(m-\frac{2)-m(\omega+1)}{4(m-1)} \rho^{-m+1}+\frac{3-a}{2} m \omega^{2} \rho^{-m-1}\right\} \sin m \omega\right. \\
& w=(3-\mu): p^{-m} \cos n \omega
\end{aligned}
$$

(iv) Same as (iii) with $\cos m \omega$ changed into $\sin m \omega$, and $\sin m \omega$ into - cos $m \omega$.

(v) $u=\rho^{-m-1} \cos m \omega$

$v=\rho^{-m-1} \sin m \omega \omega$

(vi) $\|=\rho^{-m-1} \sin m \omega$

For the force with components $P_{1}, \Omega_{1}, Z_{1}$, the coefficients of the above solutions are the following, in each case divider by $32 \pi \mu h$.

(i) $P_{1} \cos \omega_{1}-\Omega_{1} \sin \omega_{1}=X_{1}$

(ii) $P_{1} \sin \omega_{1}+\Omega_{1} \cos \omega_{1}=Y_{1}$

(iii) $\rho_{1}{ }^{m-1} \cos m \omega_{1} P_{1}-\rho_{1}{ }^{\prime \prime \prime-1} \sin m \omega_{1} \Omega_{1}$

(iv) $\rho_{1}^{m-1} \sin m \omega_{1} P_{1}+\rho_{1}{ }^{m-1} \cos m \omega_{1} \Omega_{1}$

(v) $\left\{\frac{-s m+(m+2)(\alpha+1)}{4}(\overline{m+1}) \rho_{\mathrm{I}}^{m+1}+(;-\alpha)\left(\frac{1}{2} \cdot y_{1}^{2}-\frac{1}{3} h^{2}\right) m \rho_{1}^{m-1} ; \cos m \omega_{1} \mathrm{P}_{1}\right.$

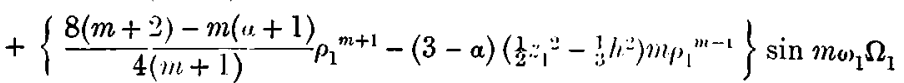

$+\quad(a-3) \rho_{1}{ }_{1}{ }^{\prime \prime}{ }_{1} \cos m \omega_{1} Z_{1}$

(vi) Sume as (v) with $\cos m \omega_{1}$ changed into $\sin m \omega_{1}$, and $\sin m \omega_{1}$ into- $\cos m \omega_{1}$. 
II. Flexural terms.

The flexural solutions are of the form given in (23), or in polur coordinates

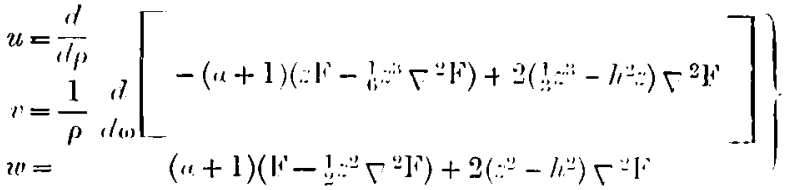

$$
\begin{aligned}
& \text { (i) } \mathrm{F}=\chi(\rho)=\frac{1}{4} r^{2} \log \frac{\rho}{2 / 2}-\frac{1}{4} r^{2} \\
& \text { (ii) } \mathrm{F}=\frac{1}{4}\left(\rho-2 \rho \log \frac{\rho}{2 / l}\right) \cos (1) \\
& \text { (iii) } \mathrm{F}=\frac{1}{4}\left(\rho-2 \rho \log \frac{\rho}{2 h}\right) \sin \omega \\
& \left.\begin{array}{l}
\text { (iv) } \mathrm{F}=\frac{1}{4 m(m-1)} \rho^{-m+"} \cos m \omega \\
\text { (v) } \mathrm{F}=\frac{1}{4 m(m-1)} \rho^{-m+2} \sin m \omega
\end{array}\right\} m>1 \text {. } \\
& \text { (vi) } \mathrm{F}=\log \frac{\rho}{2 h} \\
& \text { (vii) } \left.\mathrm{F}=\rho^{-m} \cos m(n) \quad\right\} m>0 \text {. }
\end{aligned}
$$

For the force with components $P_{1}, \Omega_{1}, Z_{1}$ the coefficients of the above solutions are the following, in each case divided by $\frac{: 2 \pi}{3} \pi h^{3}$.

$$
\begin{aligned}
& \text { (i) } Z_{1} \\
& \text { (ii) }-\ddot{\prime}_{1} \cos \omega_{1} \mathrm{P}_{1}+\ddot{n}_{1} \sin \omega_{1} \Omega_{1}+Z_{1} \rho_{1} \cos \omega_{1}=-X_{1 \mu_{1}}+Z_{1 \mu^{\prime \prime}} \\
& \text { (iii) }-z_{1} \sin \omega_{1} \mathrm{P}_{1}-z_{1} \cos \omega_{1} \Omega_{1}+Z_{1} \rho_{1} \sin \omega_{1}=-\mathrm{I}_{1 *_{1}}+Z_{1} y_{1} \\
& \text { (iv) }-{ }_{1} m \rho_{1}{ }^{m-1} \cos m \omega_{1} \mathrm{P}_{1}+{ }_{1}{ }^{m \prime \prime} p_{1}{ }^{\prime \prime \prime-1} \sin m \omega_{1} \Omega_{1}+\rho_{1}{ }^{m} \cos m \omega_{1} Z_{1} \\
& \text { (v) Same as (iv) with } \cos m \omega_{1} \text { changed to } \sin m \omega_{1} \text {, and } \sin m \omega_{1} \text { to }-\cos m \omega_{1} \\
& \text { (vi) }-\frac{1}{2}: \rho_{1} \rho_{1} \mathrm{P}_{1}+\left\{\frac{1}{4} \rho_{1}{ }^{2}+\frac{1}{3} h^{2}-\frac{1}{2} \cdots_{1}^{2}+\frac{2}{a+1}\left(z_{1}^{2}-h^{2}\right)\right\} Z_{1}
\end{aligned}
$$

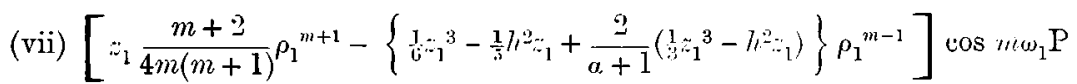

$$
\begin{aligned}
& -\left[z_{1} \frac{1}{4(m+1)} \rho^{{ }^{\prime \prime \prime+1}}-\{\quad " \quad\} \rho_{1}{ }^{\prime \prime \prime-1}\right] \sin m / \omega_{1} \Omega_{1} \\
& -\left[\frac{1}{4 m(m+1)} \rho_{1}^{{ }^{m}+"}+\left\{\frac{1}{5} h^{2}-\frac{1}{2}{ }_{1}{ }^{2}+\frac{2}{a+1}\left(z_{1}{ }^{2}-l l^{2}\right)\right\} \frac{1}{m} \rho_{1}{ }^{m}\right] \cos m \omega_{1} Z_{3}
\end{aligned}
$$

(viii) Sume as (vii) with $\cos m \omega_{1}$ changed to $\sin m \omega_{1}$ and $\sin m \omega_{1}$ to $-\cos m \omega_{1}$.

30. Types of deformation conceying a given resultent stress.

In these formulæ we remark at once a striking relation between the forms of the displacements ", ", " $"$ in the various solutions, and the multipliers of $P_{1}, \Omega_{1}, Z_{1}$ in the coefficionts of the solutions.

In I. (iii), e.g., these multi,$l i r s$ are $\rho_{1}{ }^{n-1} \cos m \omega_{1},-\rho_{1}^{m-1} \sin m \omega_{1}, 0$, which are simply the displacements of $\mathrm{I}$. (v) with sign of $m$ changed, and consequently suitable for space containing the origin.

TRANS. ROY. SOC. EDIN., VOL. XLI. PART I. (NO. 8). 
Similarly in I. ( $v$ ) the multipliers of $P_{1}, \Omega_{1}, Z_{1}$ are displacements compounded of the types (iii), (v), with sign of $m$ changed, and so on.

The full explanation of this peculiarity will be given presently, when it will be seen that an independent verification of all the results may be obtained by means of the important principle known as Betti's Theorem.

In the meantime we may examine the scheme of solutions from another very important point of view.

With reference to any individual solution, the following questions are obviously of prime importance :-

(1) What is the resultant stress transmitted!

$(2)$ Is the whole potential energy of the part of the solid bounded internally by a given cylindrical surface, finite or infinite?

Now, in order to single out those solutions which convey a finite resultant stress across any eylinder (or other surface) surrounding the origin, we have merely to look at the table of coefficients. Thus, for instance, I. (i) appears with coefficient $\mathrm{X}_{1} / 32 \pi \mu h$, from which we may infer (as verified below) that this solution conveys a stress with resultant a force of $32 \pi \mu h$ units parallel to the axis of $x$, and passing through the origin.

In this way we find that the six solutions, corresponding to the six elements which specify the resultant of a force system, are

$$
\text { I. (i), (ii), (vi) with } m=0 \text {; II. (i), (ii), (iii). }
$$

For these we shall write lown the values of the stresses $\widehat{\rho \rho}, \widehat{\rho \omega}, \widehat{\rho z}$, the components of the stress across the cylinder $\rho=$ constant.

In all, of course, we have $\approx=0$, and in I. in addition $\widehat{p}=\widehat{z=1}=0$.

$$
\begin{aligned}
& { }_{2 \mu}^{\rho \rho}=\left(\frac{a-15}{2}-\rho^{-1}+\overline{a-3} \because^{-2} \rho^{-3}\right) c(\omega) \\
& \widehat{\rho \omega}=\left(\begin{array}{c}
a+1 \\
\ddot{z} \mu
\end{array}\right.
\end{aligned}
$$

The resultant is a force along ( $),$, of magnitude

I. (ii)

$$
\begin{aligned}
& \iint(\hat{\rho} \rho \cos \omega-\hat{\rho} \omega \sin \omega) p d t \omega d x, \quad \text { talken over the cylinder } \rho \text {, } \\
& =\left(\stackrel{a-15}{2}-\frac{a+1}{2}\right) \pi \cdot 2 h \cdot 2^{2} \mu=-32 \pi \mu h .
\end{aligned}
$$

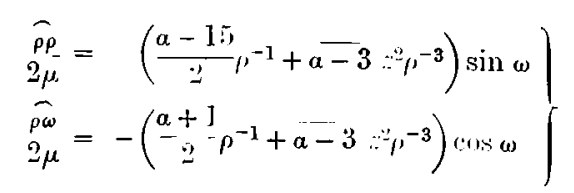

The resultant is a force along $0 y$ of milgnitude - :;2 $2 \mu h$.

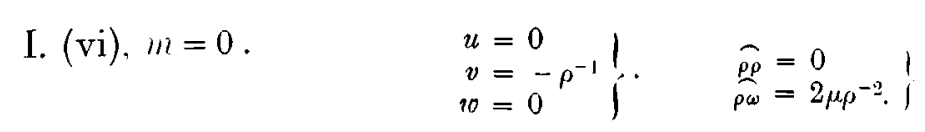


The resultant is a comple in the plano $r y$, of mannitude $8 \pi \mu l$, and we observe that the solution werms with asellicicint $-\Omega_{1} \rho_{1} / 8 \pi \mu h$.

II. The stresses in the general flexmal solution (94) are

II. (i)

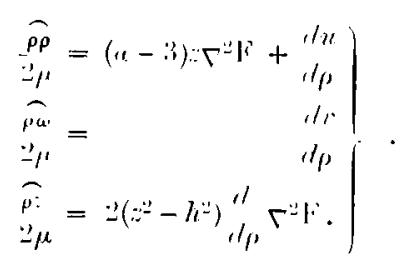

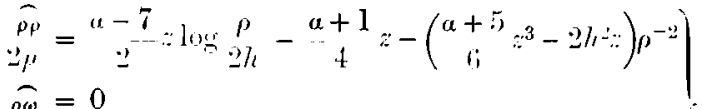

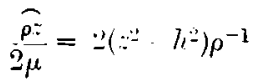

The resultant is a force along ( $)$, of magniturle $-\frac{32}{3} \pi \mu l^{3}$.

II. (ii)

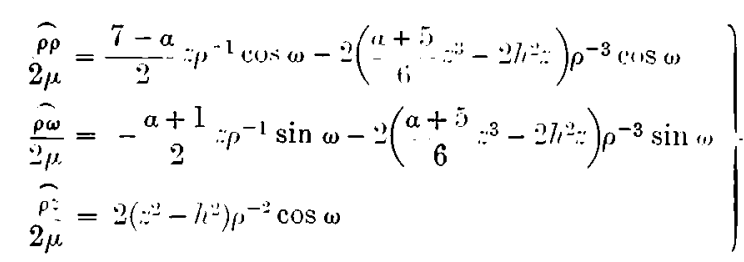

The resultant is : couple about $0 y$, of mignitude

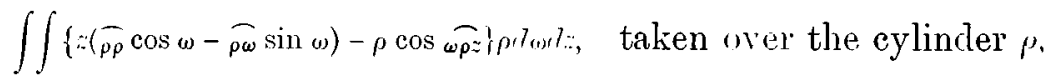

$$
\begin{aligned}
& =\frac{32}{3} \pi \mu h^{3} \text {. }
\end{aligned}
$$

II. (iii)

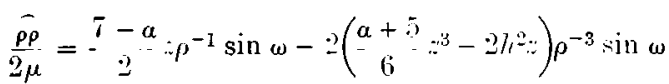

$$
\begin{aligned}
& \frac{\widehat{\rho} \omega}{2 \mu}=\frac{a+1}{2} \because \rho^{-1} \cos \omega+2\left(\begin{array}{c}
a+5 \\
6
\end{array} z^{3}-2 / l^{2} \because\right) \rho^{-3} \cos \omega
\end{aligned}
$$

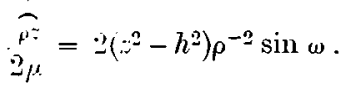

The resultant is a couple about $(U x$, of magnitule

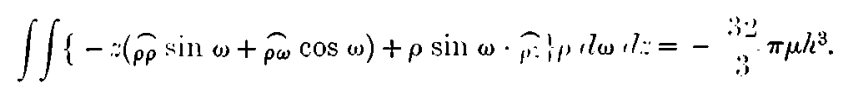

31. Conditions for the existence of a solution with funte potential energy.

Elrstive equivalence of statieally exuipollent loreds.

The corresponding results for any listribution of body force, or of traction on the faces of the plate, may be deduced at once from the alove by integration with respect to $\rho_{1}, \omega_{1}, z_{1}$ or $\rho_{1}, \omega_{1}$ with $z_{1}= \pm h$.

If the region within which the force is applied be entirely enclosed by a cylinder $\rho=a$, the results are valid for all points exterior to this cylinder. 
For a distribution of force of finite intensity per unit area or per unit volume, the potential energy of that part of the solid within the cylinder is clearly finite. The energy of the remaining part of the solid can be determined from the forms of $\S 29$.

Now, the energy between the cylinders $\rho=a, \rho=l$ is the integral of

$$
\stackrel{2}{2}(u \hat{\rho \rho}+n \hat{\rho \omega}+u \cdot \hat{\rho} \bar{z})
$$

taken over the belt of the cylinder $\rho=\ell$ cut off by the plate, diminished by the corresponding integral for $\rho=a$. Hence the condition of finiteness of the whole potential energy is simply that the value of the integral for the surface $\rho=\mu$ tends to zero as $p$ tends to infinity. 'This conlition is obviously satisfied by all the partial solutions of $\S \supseteq 9$, except those which have been already singled out as conveying a finite resultant stress. It is also satisfied by one of the latter lass, namely, that which conveys a couple in the plane of the plate.

Hence, when force is ipplied to a circumscribed portion of the solid, a solution giving finite potential energy will exist provided the force either constitutes an equilibrating system, or reduces to a couple in the plane of the plate. It does not follow, however, even for an equilibrating application of force, that a solution will exist giving vanishing displacements at infinity. We need only point to the solutions of $\$ 29$, II. (vi) and (iv), (v) with $m=2$. This being so, it may be of interest to write down a few more detuils of those solutions which rank in importance next to the solutions of finite resultant stress.

I. (iii) with $m=2$.

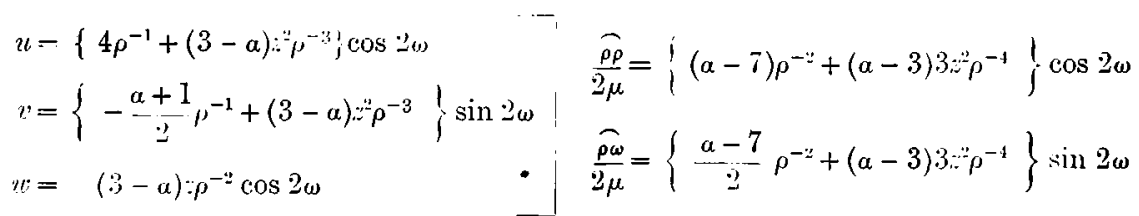

This solution occurs with coefficient $\left(\mathrm{X}_{1} x_{1}-\mathrm{Y}_{1} z_{1}\right) / 32 \pi \mu h$.

I. (iv) with $m=2$ is obtained by writing $\sin 2 \omega,-\cos 2 \omega$ for $\cos 2 \omega, \sin 2 \omega$ in the preceding, and the coefficient is $\left(\mathrm{X}_{1} y_{1}+\mathrm{Y}_{1}, r_{1}\right) / 32 \pi \mu / /$.

I. (v) with $m=0$.

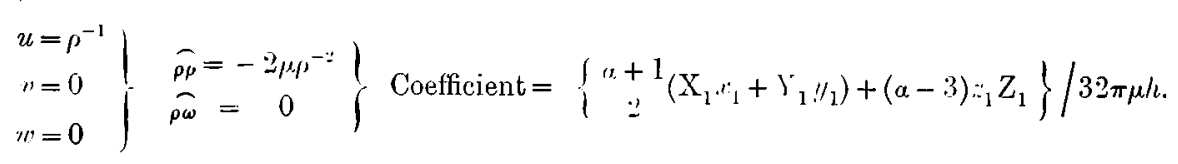

II. (iv) with $n=2$. F $=\frac{1}{5} \cos 2 \omega$.

$$
\begin{aligned}
& u=\left(\begin{array}{c}
a+5) \\
6
\end{array} ;-2, \cdots\right) \rho^{-3} \cos 2 \omega
\end{aligned}
$$

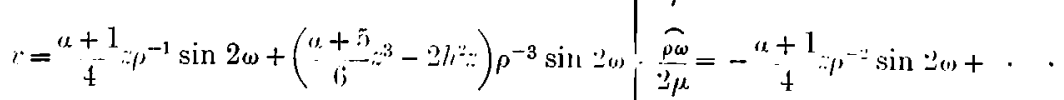

$$
\begin{aligned}
& w=\frac{a+1}{i} \cos 2(1)+\left(\begin{array}{c}
(1)-2+2 \\
4
\end{array}\right.
\end{aligned}
$$


The cocthiciont is

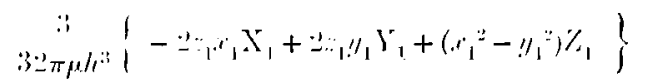

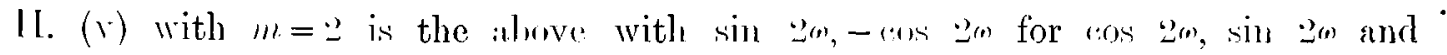
rocthicient

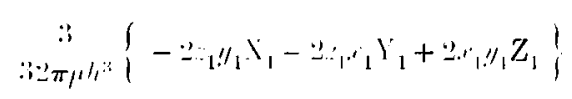

I1. (vi). $\mathrm{F}=\log _{2,1 /}$.

Coetticient is

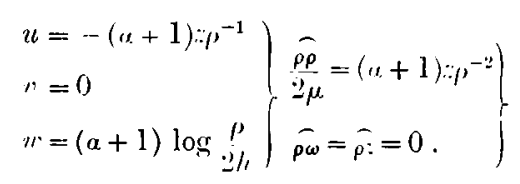

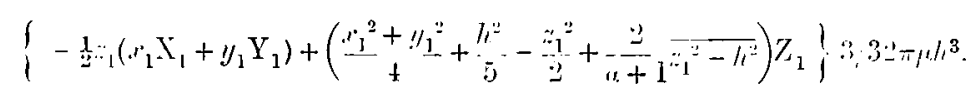

For all the remaining solutions, the stresses are of the third or higher order in $1 / \rho$, The results of this and the preceling irtirle lear directly upon a principle of fundamental importance in theories of approximation, generaily referred to as the principle of the rlastic crumerence of stretically equipollent systems of lored, and a study of these results will be found of service in imparting precision and definiteness to one's view of the principle in its application to the theory of plates.

It may be noted here, with reference to the occurrence of the function log $(\rho / 2 h)$ in some of the principal solutions of $\$ 30$, that it would make no essential difference if this function were replareel throughout by $\log (\rho, c), c$ being any length whatever, the unit of length for example. 'The change would be equivalent to adding a solution of the permanent type, giving no body force or traction on the faces, and it will be observed that the addition would disappear altogether when the applied forces are in equilibrium.

We have here, in fact, an instance of the indeterminateness that of necessity arises in the absence of conditions at infinity, :mul we are thus brought to the question, what is the exact extent of this indeterminateness? or, as it may be put, given one solution of a problem sitisfying the conditions at a finite rlistance, what is the most general solution sitifying surh conditions?

For the investigation of this "question we have at hand a porrerful instrument in Betti.s Theoreme, which oceupies in the theory of elastic solids the place held by Green's Theorem in the Therry of the Potential.

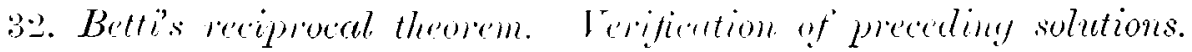

Betti's Therem may be thus stated:- diven two sets of displacements of an elastic solid, with the two rorresponding sets of forces maintaining these displacements (including body forees, surfice tractions, and linetic reactions), then the work done by the forces of the first set acting on the displacements of the second set is 
equal to the work done by the forces of the second set acting over the displacements of the first.

In potential theory one of the chief applications of Green's Theorem is to the case when one of the potential systems includes a mass concentrater at a single point, and in the present subject Betti's Theorem finds an application of like importance when one of the displacement systems contains a finite force applied at one point, or, in analytical language, includes a point singularity of the first order, that is to say, of one of the three forms indicated in (6).

Thus, let us suppose the solid to be bounded hy a surface $s$, and in the first set let the displacements be $u, v, w$; the components of body foree per unit volume $X, Y, Z$; and the components of the traction on $\mathrm{S}, \mathrm{F}, \mathrm{H}, \mathrm{H}$; in the second set let the displacements be $u^{\prime}, v^{\prime}, w^{\prime}$; the only internal force a force $\mathrm{X}^{\prime}, \mathrm{Y}^{\prime}, \mathrm{Z}^{\prime}$ at $\left(x^{\prime}, y^{\prime}, z^{\prime}\right)$, and the tractions on $S, \mathrm{~F}^{\prime}, \mathrm{G}^{\prime}, \mathrm{H}^{\prime}$.

We may apply Betti's Theorem to the space bounded by $S$ ' and a sphere $S^{\prime}$ of radius $\epsilon$ drawn round $\left(x^{\prime}, y^{\prime}, z^{\prime}\right)$ as centre. Thus w have

$$
\begin{aligned}
& \iiint\left(\mathrm{X} u^{\prime}+\mathrm{Y}^{\prime} v^{\prime}+\mathrm{Z} u u^{\prime}\right) d \mathrm{~V}+\iint\left(\mathrm{F} u^{\prime}+\left(u^{\prime} r^{\prime}+\mathrm{H} u^{\prime}\right) d s^{\prime}+\iint\left(\mathrm{F} u u^{\prime}+\mathrm{G} u^{\prime}+\mathrm{H} u^{\prime}\right) d \mathrm{~S}^{\prime}\right. \\
& =\iint\left(\mathrm{F}^{\prime} u+\left(\mathrm{r}^{\prime} x+\mathrm{H}^{\prime} u\right) d \mathrm{~S}+\iint\left(\mathrm{F}^{\prime} u+\left(\dot{r}^{\prime} v+\mathrm{H}^{\prime} u\right)+\mathbb{S}^{\prime} .\right.\right.
\end{aligned}
$$

Now take the limits of both members of this equality for $\epsilon=0$.

Since near the centre of the sphere $S^{\prime}, u^{\prime}, \iota^{\prime}, u^{\prime}$ are of order $1 / \epsilon, F^{\prime}, c^{\prime}, H^{\prime}$ of order $1 / \epsilon^{2}$, and $1 \boldsymbol{S}^{\prime \prime}$ of order $\epsilon^{2}$, the effect on the volume integral is simply to extend it to the whole volume within $S$; the surface integral $\iint\left(\mathrm{F} u u^{\prime}+\left(u v^{\prime}+\mathrm{H} v^{\prime}\right) d \mathrm{~S}^{\prime}\right.$ vanishes, and the surface integral $\iint\left(\mathrm{F}^{\prime} u+\left(\mathrm{G}^{\prime} v+\mathrm{H}^{\prime} u\right) d \mathrm{~s}^{\prime}\right.$ has the silme limit as

namely,

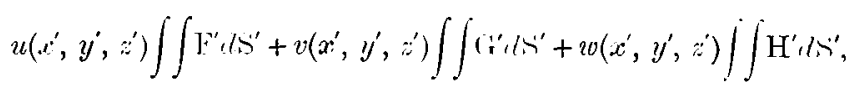

the tractions $\mathrm{F}^{\prime}, \mathrm{G}^{\prime}, \mathrm{H}^{\prime}$ on $\mathrm{S}^{\prime}$ being statically equivalent to the force $\mathrm{X}^{\prime}, \mathrm{I}^{\prime}, Z^{\prime}$ at its centre.

It is thus apparent, and might imlend have been anticipated, that Betti's Theorem may legitimately be applied when one of the systems contains a force acting at a single point, provided the work done by this force on the other system of displacements be taken into account.

The theorem thus becomes

$$
\begin{aligned}
& \iiint\left(\mathrm{X} u^{\prime}+\mathrm{Y} v^{\prime}+Z^{\prime} w^{\prime}\right) d V+\iint\left(\mathrm{F} u^{\prime}+\left(\mathrm{i}^{\prime} v^{\prime}+\mathrm{H} w^{\prime}\right) d \mathrm{~S}-\iint\left(\mathrm{F}^{\prime} u+\mathrm{G}^{\prime} v+\mathrm{II} w\right) d \mathrm{~S}\right. \\
& \left.=u\left(x^{\prime}, y^{\prime}, z^{\prime}\right) \mathrm{X}^{\prime}+\cdots\left(x^{\prime}, y^{\prime}, a^{\prime}\right)\right)^{\prime \prime}+w\left(x^{\prime}, y^{\prime}, z^{\prime}\right) z^{\prime}
\end{aligned}
$$

In order to :pply the theorem to the plate probloms under disenssion, take for the solid a portion of the plate bounded externally by any orthogonal cylimlcr. Let us 


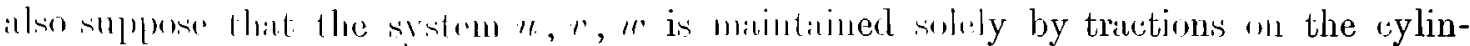

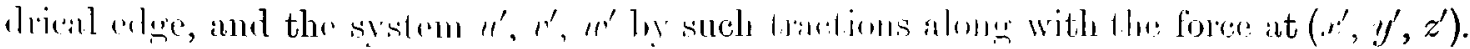
Further, it will be convenient to derompese the latice system, and take $u_{1}, n_{1}, w_{1}$ as due

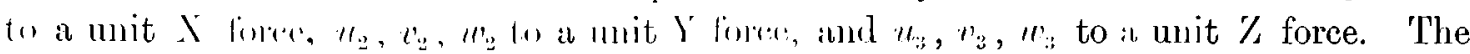

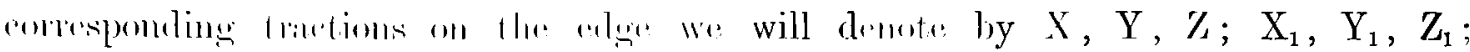
$X_{2}, Y_{2}, Z_{2} ; X_{i}, Y_{3}, Z_{3}$. I'lue theorem (96i) then gives

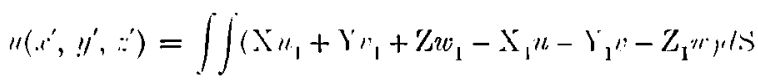

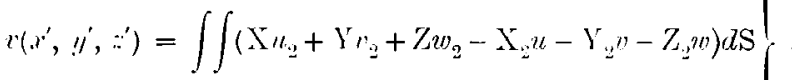

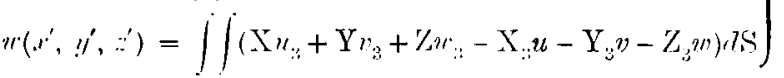

the integrals being taken wer the edge.

Is onc application of these forms, we may inlicate briefly how they can be used to verify the single force solutions alrcarly obtained.

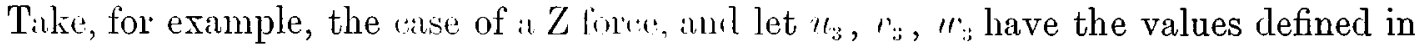
(i3), (64), (65). Mso let the edge be the cylinulur $\mathrm{R}=$ constant.

(i) The coefficient of the principal flexural term, in which, with the notation of (94) $F \infty \chi(R)$, is determiner from the comlition that the resultant of the stress $\pi_{i}$ must balanre the applied fores.

It is interesting to note that the conditions of equilibrium of applied fores and surface tractions may he regarted as sperial cases of Betti's Theorem. We have only to talie for auxiliary systems the rigid body displacements $u=0, v=0, u=1 ; u=y$, $r=-x, w=0$, etc.

(ii) In the third of equations (9r) take for $", ", "$ the values of $(94)$ with $F=R^{2}$. Only the two Hexural terms of (65) contribute to the surface integral; the contribution from the particular solution $\phi=G_{0} \kappa \mathrm{R} \sinh \kappa ;, \theta=-\cosh 2 k h \cdot \phi$ must vanish, as we see by pushing the elge to infinity.

'This, with the result of (i), gives the coeficient of the second flexural term of (65).

(iii) The principal extensional term is rerifierl by taking

$$
u=\frac{a+1}{3}\left(x-x^{\prime}\right), \quad n=\stackrel{a+1}{y}\left(y-y^{\prime}\right), \quad u=(a-3) z .
$$

(iv) The coetficient of the particular solution $\phi=\mathrm{C}_{\mathrm{r}}, \mathrm{R} \sinh \kappa * \theta, \theta=-\cosh 2 \kappa h \cdot \phi$ in (693) is verified by taking for $\|, n, w$ the values defined by $\phi=\mathrm{J}_{0} \mathrm{R} \sinh \kappa z$, $\theta=-\cosh 2 \kappa l \cdot \phi$.

None of the solutions composmling to the other roots of $\sinh 2 \kappa h-2 \kappa h$ contribute to the surface integral. In fact, the pirtial contribution from a root $\kappa^{\prime}$ being independent of the radius of the cylinder, must vinish identically, since the Bessel Functions supply in factor twuling to \%oro or infinity when $\mathrm{R}$ is marle infinite, according as $\kappa^{\prime}$ is a higher or lower root than $\kappa$.

(v) The coefficient of the particular solution $\phi=\mathrm{G}_{0}{ } \mathrm{R} \cosh \kappa \approx, \theta=\cosh 2 \kappa \cdot l \cdot \phi$, may be verified in the same way. 
It is now ensy to see the significance of the forms of the coetticients in the solutions of $\S 29$ and the confirmation of the values there given would obviously present no difficulties.

33. Finite plate under edge tractions. Form of the solution deduced by means of Bettis Theorem.

We pass, however, to a more important application of the theorems (97). The system $u, v, w$ we still suppose maintained by erge tractions alone, but in addition to the external edge the solid may now be bounded by one or more internal edges. For $u_{1}, v_{1}, u_{1}$, etc., we take the definite values defined in (79), (82), (83), and in (63), (64), (65).

Thus in (97) $u_{1}, v_{1}, w_{1}, \mathrm{X}_{1}, \mathrm{Y}_{1}, Z_{1}$, and the other displacements and tractions marked with suftixes, are known functions of $x^{\prime}, y^{\prime}, z^{\prime}$, and the equations give explicitly the values of the displacements at any internal point in terms of the risplacement and stress at the edge or edges.

The ideal solution would give the internal displacement in terms of edge displacement alone, or of erge stress alone, but the analytical difficulties are such that we are unable to solve the problem thus completely even for the simplest case, that of a single infinite plane edge. Meantime, however, we may derive valuable information from the expressions of (97), and in the first place as to the form into which any solution due to edge tractions alone may be thrown.

Just as in the case of the original source solutions, we find that the solution, in which, of course, the accentel letters are now the variables, may be decomposed into an extensional and a flexural part, while in each of those parts we may separate a permanent mode from an infinite series of transitory or decaying moles of two types, the $\psi$ type, characterised by no dilatation or normal displacement, and the $\theta$, $\phi$ type, in which there is no molecular rotation in the planc: of the plate.

In the following inalysis integrals of the same form is those in (97) oceur frequently; the system $u, v, w$ appearing in each aise, but associated with various other systems. For conciseness we shall refer to the first integrial of (97) ils the $1 \%$ or difference from $u_{1}, v_{1}, w_{1}$, and similarly in other miscr.

I. Extensional part of the silution.

(i) Permanent mode.

In $u_{1}, v_{1}, w_{1}$, the term: which relite to this mole are the unambigunus terms, even in $z$, of $(83)$, after these have bern divicled hy $4 \pi \mu(a+1)$. These, as maly be seen from a glance at the beginning of $\$-7$, an cruivalent to

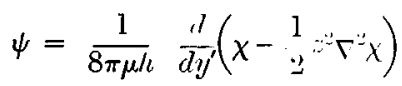

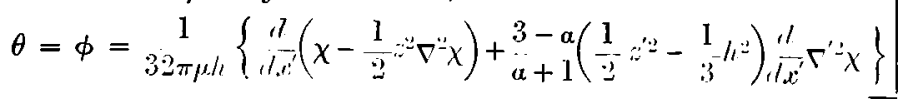

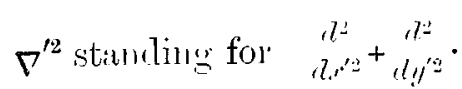


Now let the work difference from the system $\psi=\frac{1}{8 \pi \mu h}\left(x-\frac{1}{2} \cdot v^{2} \nabla^{2}\right)$ he denoted by $\mathrm{E}_{1}$, and that from the system $\theta=\phi=\frac{1}{32 \pi \mu l}\left(x-\frac{1}{2}:^{a} \gamma^{2} x\right)$ ly $\mathrm{E}_{2}$; then obviously the work differences from the two systems immediately precerling are respectively

Hence $\left.(9)^{-}\right)$gives

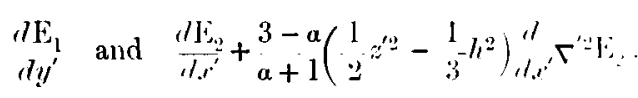

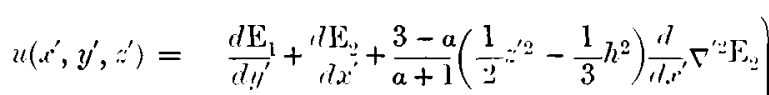

In the same way from $u_{2}, v_{2}, w_{2}$ and $u_{3}, v_{3}, w_{3}$ we obtain

$$
\begin{aligned}
& r\left(x^{\prime}, l^{\prime}, z^{\prime}\right)=-\frac{d \mathrm{E}_{1}}{d l t^{\prime}}+\frac{d \mathrm{E}_{3}}{d y^{\prime}}+\frac{3-a}{a+1}\left(\frac{1}{2} y^{\prime \prime 2}-\frac{1}{3} l^{2}\right) \frac{d}{d y^{\prime}} \Gamma^{\prime \prime}-\mathrm{E}_{2} \\
& r\left(x^{\prime} y^{\prime}, \because^{\prime}\right)=\frac{a-3}{a+1} \dot{\prime}^{\prime} \Gamma^{\prime 2} \mathrm{E}_{2}
\end{aligned}
$$

Moreover, it can be seen in il moment that the displacements due to

$$
\psi=\frac{d}{d y} \gamma^{\prime \prime \prime} \chi \text { and to } \theta=\phi=-\frac{2}{a+1} \frac{d}{d x^{\prime}} \Gamma^{2} x
$$

are in reality the same; as also those due to

$$
\psi=\frac{d}{\lambda x^{\prime}} \nabla^{\prime \prime} \chi \text { and to } \theta=\phi=\frac{2}{a+1} \frac{d}{d ! l^{\prime}} \nabla^{\prime} \cdot \chi .
$$

It follows that

$$
\text { and } \left.\begin{array}{r}
\frac{d}{d y^{\prime}} \Gamma^{\prime \prime} \mathrm{E}_{1}+\frac{8}{a+1} \frac{d}{d x^{\prime}} r^{2} \mathrm{E}_{2}=0 \\
\frac{d}{d x^{\prime}} r^{\prime \prime 2} \mathrm{E}_{1}-\frac{8}{a+1} \frac{d}{d y^{\prime}} r^{\prime 2} \mathrm{E}_{2}=0
\end{array}\right\}
$$

If we write $\mathrm{U}$ for $\quad \frac{d \mathrm{E}_{1}}{\lambda ! !^{\prime}}+\frac{\lambda \mathrm{E}_{2}}{d x^{\prime}}-\frac{3-a}{a+1} \frac{1}{3} l t^{2} \frac{d}{d r^{\prime}} \nabla^{\prime 2} \mathrm{E}_{2}$,

and $\mathrm{V}$ for

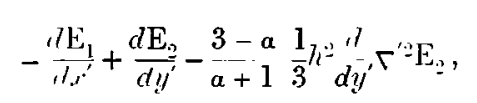

we obtain the form which it is convenient to take as the standard for this kind of strain, namely,

with

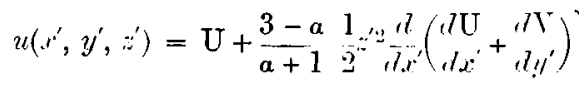

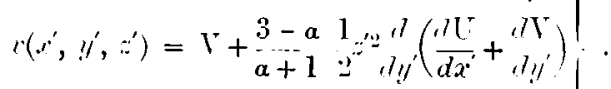

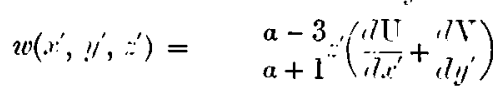

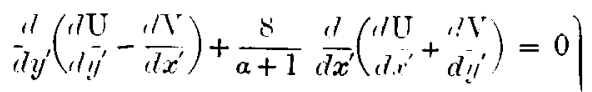

$$
\begin{aligned}
& \frac{d}{d x^{\prime}}\left(\frac{d \mathrm{U}}{d y^{\prime}}-\frac{d \mathrm{~V}}{d x}\right)-\frac{8}{a+1} \frac{d}{d ! \prime}\left(\frac{d \mathrm{U}}{d y^{\prime}}+\frac{d \mathrm{~V}}{d y^{\prime}}\right)=0
\end{aligned}
$$

TRANS. ROY. SOC. EDIN., VOL. XLI. PART I. (NO. 8). 
(ii) Transitory modes, $\psi$ or rotational type.

Referring to the expression for $\psi^{\prime}$ in (82), put

$$
\mathrm{E}_{3} \equiv \text { work difference from the system } \psi=\frac{1}{8 \pi \mu \kappa^{2} h} \cosh \kappa: \mathrm{T}_{0} \kappa \mathrm{R} \text {. }
$$

Then for this part of the solution

$$
\left.\begin{array}{l}
u\left(x^{\prime}, y^{\prime}, y^{\prime}\right)=\sum_{\kappa} 2 \frac{d \mathrm{E}_{3}}{d y^{\prime}} \cosh \kappa:^{\prime} \\
r\left(x, y^{\prime}, y^{\prime}\right)=\sum_{\kappa}(-2) \frac{\prime E_{*}}{d \cdot x^{\prime}} \cosh \kappa z^{\prime} \\
u\left(x^{\prime}, y^{\prime}, z^{\prime}\right)=0
\end{array}\right\}
$$

where $\kappa$ is a pos. imag. root of $\sinh \kappa h$, and $\frac{d^{2} \mathrm{E}_{3}}{d x^{\prime 2}}+\frac{d^{2} \mathrm{E}_{3}}{d y^{\prime 2}}+\kappa^{2} \mathrm{E}_{3}=0$.

The solutions here are obviously of the type $\psi=\cosh \kappa z^{\prime} \mathrm{E}_{3}\left(x^{\prime}, y^{\prime}\right)$.

(iii) 'Transitory modes, $\theta-\phi$ or dilatational type.

Looking to (64), (8*), put

$\mathrm{E}_{4} \equiv$ work difference from the system $\left\{\begin{array}{l}\phi=\frac{\cosh \kappa z \mathrm{G}_{0} \kappa \mathrm{R}}{\kappa \pi \bar{\mu}(a+1) \kappa^{2} h(\cosh \overline{2 \kappa h+1})} \\ \theta=\cosh 2 \kappa h \cdot \phi\end{array}\right.$

Then

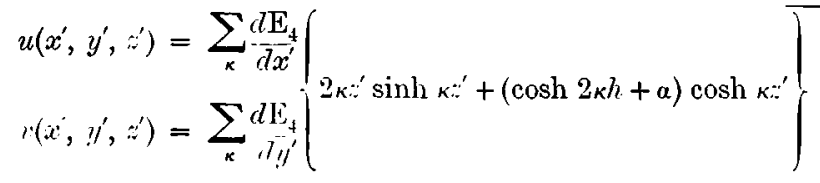

$$
\begin{aligned}
& u\left(x^{\prime}, y^{\prime}, \varkappa^{\prime}\right)=\sum_{\kappa} \kappa \mathrm{E}_{4}\left\{2_{\kappa z^{\prime}} \cosh \kappa z^{\prime}+(\cosh 2 \kappa h-\alpha) \sinh \kappa z^{\prime}\right\}
\end{aligned}
$$

where $\kappa$ is a zero of $\sinh 2 \kappa h+2 \kappa h$ with pos. imag. part, and $\frac{d^{2} \mathrm{E}_{4}}{d x^{23}}+\frac{d^{2} \mathrm{E}_{4}}{d y^{2}}+\kappa^{2} \mathrm{E}_{4}=0$.

The solutions are of the type $\phi=\cosh \kappa z^{\prime} \mathrm{E}_{4}\left(x^{\prime}, y^{\prime}\right), \theta=\cosh 2 \kappa h \cdot \phi$.

II. Flexural part of the solution.

(i) Permanent mode.

Let $F_{1} \equiv$ work difference from the system

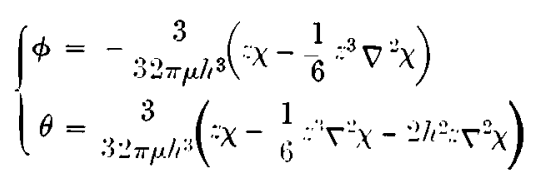

Then $\nabla^{\prime 2} \mathrm{~F}_{1}=$ work difference from $\phi=-\underset{32 \pi \mu l^{\prime}}{3} z \nabla^{2} \chi=-\theta$,

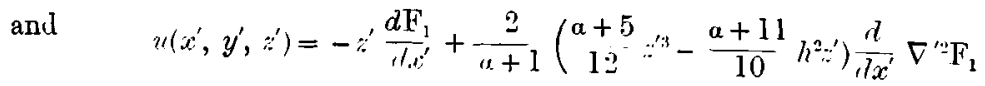

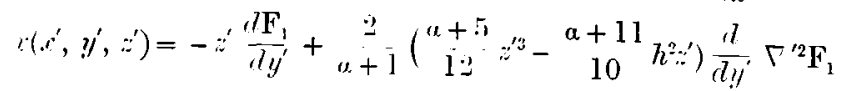

$$
\begin{aligned}
& w\left(x^{\prime}, y^{\prime}, z^{\prime}\right)=\mathbf{F}_{2}+\left\{\frac{1}{n} / l^{\prime \prime}-\frac{1}{2} y^{\prime \prime}+\frac{2}{a+1}\left(z^{\prime \prime}-h^{2}\right)\right\} \Gamma^{\prime \prime} \mathrm{F}_{1}
\end{aligned}
$$


Here $\nabla^{\prime \prime} \mathrm{F}_{1}=0$, and if we write $(a+1) \mathrm{F}$ for $\mathrm{F}_{1}+\frac{1}{n} l^{\prime} \nabla^{\prime \prime \prime} \mathrm{F}_{1}$, the expessins reduce to the form which we hilve talken throughout for this kind of strain, namely.

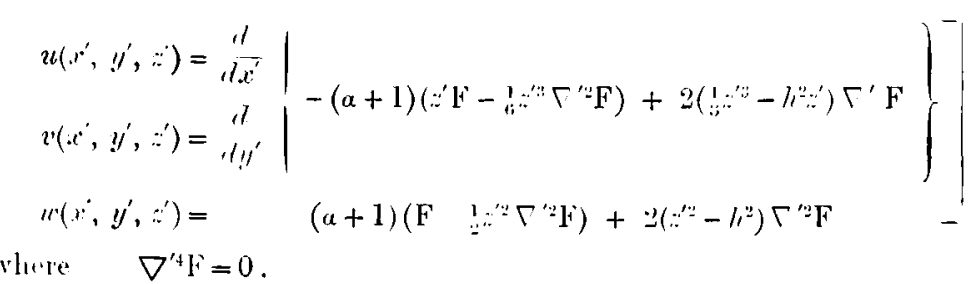

(ii) 'Transitory moles, $\psi$ or rotational type.

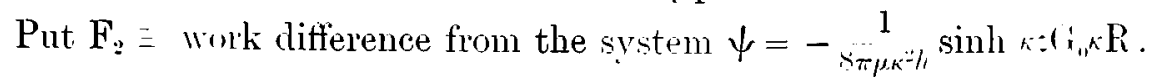

$$
\text { Then } \quad \begin{aligned}
u\left(x^{\prime}, y^{\prime}, y^{\prime}\right) & =\sum_{\kappa} 2 \frac{d \mathrm{~F}_{2}}{d y} \sinh \kappa !^{\prime} \\
r\left(x^{\prime}, y^{\prime}, y^{\prime}\right) & =\sum_{\kappa}(-2) \frac{d \mathrm{~F}_{2}}{d l^{\prime}} \sinh \kappa !^{\prime} \\
& u\left(x^{\prime}, y^{\prime}, y^{\prime}\right)=0
\end{aligned}
$$

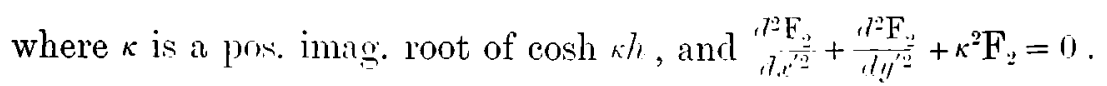

The solutions are of the type $\psi=\sinh k z^{\prime} \mathrm{F}_{2}\left(x^{\prime}, y^{\prime}\right)$.

(iii) Transitory morles, $\theta-\phi$ or dilatational trpe.

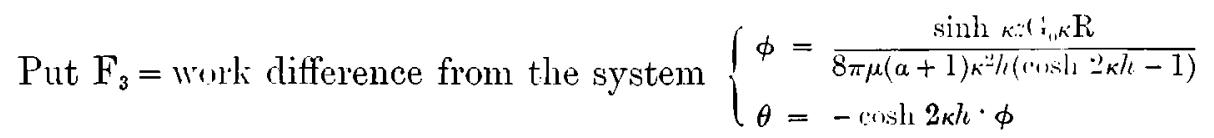

Then

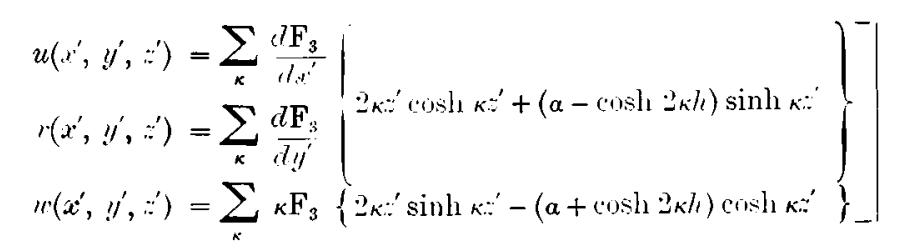

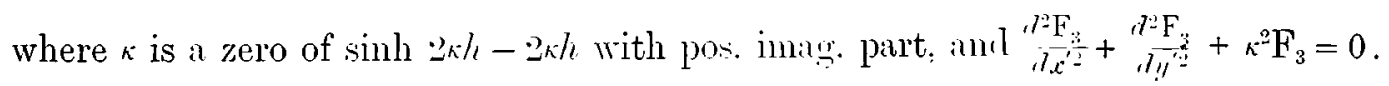

The solutions are of the type $t^{\prime}=\sinh \kappa z^{\prime} \mathrm{F}_{3}\left(l^{\prime}, y^{\prime}\right), \theta=-\cos h_{2} 2_{\kappa} i^{\prime} \cdot \phi$.

34. Form of the solution for edge tructions deduced by another method.

We have thus shown that the most general deformation of a finite plate under edge tractions only is compounded of the types specifiel in (98) . . (103). The deformation is of the same form as that given by our infinite plate solutions for any part of the solid free from bolly force or surface traction, and it may be of advantage to show in a direct manner why this should be so.

Suppose, then, that we have given a displacement $(u, v, w)$ of a finite plate bounded by an external edge $S$ and one or more internal edges $S^{\prime}$, the only applied forces being tractions on the edges. Imagine the plate continued inwards and outwards so as to 
form a complete infinite plate. By the general existence theorem of the subject, there exist values of $u, v, w$ in the space within an internal edge, continuous at the edge with the values of the displacenents of the original solid, and produced by edge tractions alone. Similarly, if we take any surface $\mathrm{S}^{\prime \prime}$, within the infinite plate, but completely enclosing the erge $S$, there exist ralnes of $", n$, " continuous with the original $u, v, w$ at the external adge, and hecoming zero on $\mathrm{S}^{\prime \prime}$; these "liso being prorluced by edge tractions only, namely, on $\mathrm{s}$ and $\mathrm{S}^{\prime \prime}$.

If, then, we take $u, v, w$ to be zero outside $\mathrm{s}^{\prime \prime}$, we olitiin altogether a system of displacements continuous throughout the infinite solid. The forces required to maintain this system are given lirectly ly the general equations of equilibrium. These forces form areal distributions on $\mathrm{S}, \mathrm{S}^{\prime}, \mathrm{S}^{\prime \prime}$, and are measurerl by the discontinuity of stress at these surfaces. Further, on the whole they make up an equilibrating system. But we have shown in the preceding paiges how to find a solution for such a system of force, this solution giring displacements of order $\log \mathrm{R}$ at most, and stresses of order $\mathrm{R}^{-2}$ at most, at a great distance. Only one solution fulfilling these conditions being possible, our solution is the solution.

Hence, finally, any displacement of a finite plate under edge tractions only is of the same form as that given by our infinite solid solutions for a certain system of areal force, distributed partly over the edges, and partly over an arbitrary external surface. This is what we proposed to prove.

\section{General solution for an infinite solid under any forces.}

It is now easy to determine the most general form of displacement of an infinite solid, under null borly force and fire tration, and free from singularity at a finite distance. For if $u, c, w$ be any such displacement, then within any surfice $\mathbb{S}$, however distant, we have proved that $u, n, w$ are given by the absolutely convergent series (98) .... (10:3).

If we take a right circular cylinder for the surface $s$, the functions $\mathrm{F}$ which satisfy equations of the form $\frac{d^{2} \mathrm{~F}}{d x^{2}}+\frac{d t^{\prime \prime} \mathrm{F}}{\left.d \eta^{2}\right|^{2}}+\kappa^{2} \mathrm{~F}=0$ can be expressed in series of the form

$$
\sum_{m} \mathrm{~J}_{m} \kappa \rho\left(\Lambda_{m} \cos m(m)+\mathrm{B}_{m} \sin m \omega\right)
$$

and the only restriction on the coetticients $A_{m}, B_{m}$ is that they must make the double series in which the complete solution is thus expressed absolutely convergent for all ralues of $\rho$, however great.

The most general solution for any system of force ipplied at a finite distance is of course obtained by adding to this complete free wolution the particular wolution already investigated. It may be observel that this final result might have been obtained in one step by the process of 53 , if in that article we had taken for $u, v, w$ any displacements under given borly frorce and surface traction, insteirl of under edge traction only. The identity of the results of the two methorls will be seen to depend essentially on the 
fact that in the solution for a single firce in any direetion, the component displacement

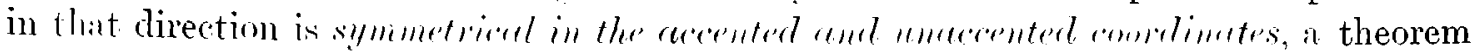
anileguns to a well-known property of Green's function in Potential Theory.

It is interesting to olwerve that, in the process suggesterl in the last sentence but

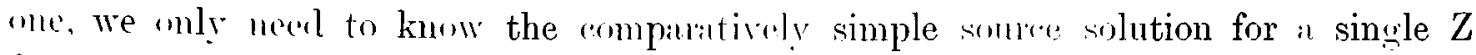

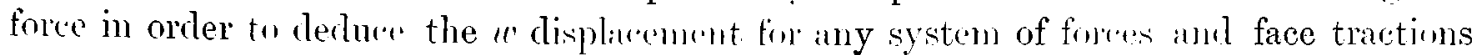
whatever.

\section{Ippliation of Bett's Theorem to the problem of given entge tractions.}

In the remaining pares, we shall be occupied almost exclusively with deformations of a finite plate under elge tractions only. For brevity wo may refer to such deformations as fiee.

The formule (97) express the internal displacements in terms of the erlye displicements ami edge tractions. We may indicate here the general lines along which we naturally proceer in the attempt to reduce these formulæ to expressions in terms of displacements alone or of tratetions alone.

Taking the first equation of (97), for example, if we wish a formula containing edge displacements only, we look fir free displacements in the form of functions $u_{1}^{\prime}, v_{1}^{\prime} . w_{1}^{\prime}$ of $x, !, z$, such that $u_{1}+u_{1}^{\prime}, v_{1}+u_{1}^{\prime}, u_{1}+w_{1}^{\prime}$ shall be equal to zero at the edge.

If $\mathrm{Y}_{1}{ }^{\prime}, \mathrm{Y}_{1}{ }^{\prime}, \mathrm{Z}_{1}{ }^{\prime}$ le the edge tractions in the system $u_{1}{ }^{\prime}, r_{1}{ }^{\prime}, u_{1}{ }^{\prime}$, then by Betti's Theorem

$$
\iint\left(\mathrm{X} u_{1}^{\prime}+\mathrm{Y} u_{1}^{\prime}+Z w_{1}^{\prime}-\mathrm{X}_{1}^{\prime} u-\mathrm{Y}_{1}^{\prime}{ }^{\prime}-Z_{1}^{\prime} w^{\prime}\right) d \bar{s}=0
$$

and by addition of this equation to $(97)$,

$$
u\left(x^{\prime}, y^{\prime},:^{\prime}\right)=-\iint\left\{u\left(\mathrm{X}_{1}+\mathrm{X}_{1}{ }^{\prime}\right)+v\left(\mathrm{Y}_{1}+\mathrm{Y}_{1}^{\prime}\right)+w\left(Z_{1}+Z_{1}{ }^{\prime}\right)\right\} d \mathrm{~S} .
$$

The problem of arbitrary edge displacements is thus reduced to a problem in which these displacements have a comparatively simple form.

When we attempt to find a formula in terms of erge tractions only, the procedure is not quite so simple, in consequence of the fact that the tractions $\mathrm{X}_{1}, \mathrm{Y}_{1}, \mathrm{Z}_{1}$ are not equilibratiug, but equivalent to a negative unit $\mathrm{X}$ force through $\left(x^{\prime}, y^{\prime}, z^{\prime}\right)$. From various methorls of meeting this difficulty we select the following as the most convenient in the present case.

We have seen in $\$ 0$ that the system $\imath_{1}, v_{1}, u_{1}$ can be decomposed into four systems. The first system, say $\mathrm{l}_{1}, V_{1}, W_{1}$ convers no resultant stress; the second system conveys a stross equivalent to a unit $\mathrm{X}$ force through the origin, and the displacements are independent of $x^{\prime}, y^{\prime}, z^{\prime}$; the third system conveys a couple $z^{\prime}$ in the plane $z 0 x$, the displacements contain $z^{\prime}$ as it factor, but are otherwise independent of $r^{\prime} . y^{\prime}, z^{\prime}$; the fourth system conveys a couple $-y^{\prime}$ in the plane $x(y y$, the displacements involving $x^{\prime}, y^{\prime}, z^{\prime}$ only in the form of the fictor $y^{\prime}$. 
The displacements $u_{2}, v_{2}, v_{2}$ and $u_{;}, v_{j}, u_{3}$ are similarly decomposable into equilibrating systems $\mathrm{U}_{2}, \mathrm{~V}_{2}, \mathrm{~W}_{2}$ and $\mathrm{U}_{3}, \mathrm{~V}_{3}, \mathrm{~W}_{3}$ with other systems conveying resultant forces ancl couples. The contributions to $u\left(x^{\prime}, y^{\prime}, z^{\prime}\right)$. $u^{\prime}\left(x^{\prime}, y^{\prime}, z^{\prime}\right), w^{\prime}\left(x^{\prime}, y^{\prime}, z^{\prime}\right)$ in $(97)$ from the various systems conveying forces and couples amount on the whole merely to a rigid borly displacement of the plate. If this be neglected, then the value of $u\left(x^{\prime}, y^{\prime}, z^{\prime}\right)$, for instance, becomes simply the work difference from the srstem $U_{1}, V_{1}, W_{1}$, the edge tractions due to which are equililırating, and can be balanced by a free system. $\mathrm{U}_{1}^{\prime}, \mathrm{V}_{1}^{\prime}, \mathrm{W}_{1}^{\prime}$. We then obtain from $(97)$

$$
u\left(x^{\prime}, y^{\prime}, z^{\prime}\right)=\iint\left\{\mathrm{X}\left(\mathrm{U}_{1}+\mathrm{U}_{1}^{\prime}\right)+\mathrm{Y}\left(\mathrm{V}_{1}+\mathrm{V}_{1}^{\prime}\right)+Z\left(\mathrm{~W}_{1}+\mathrm{W}_{1}^{\prime}\right)\right\}, d \mathrm{~S}
$$

and similarly for $v, u$.

37. Exact solutions of special problems for a circular plrte.

As already stated, we are not at present in a position to complete the solution of the problem of arbitrary edge tractions, even for the simplest form of edge. The method just indicated may be used, however, whatever be the form of the edge, to obtain approximately the boundary conditions which define the permanent part of the solution. But before entering on this important application, we shall consider a few special problems which admit of exact solution. All of these have reference to a plate bounded by a right circular cylinder, with or without a concentric circular aperture, and to systems of displacement symmetrical about the axis.

The radius of the external edge is $a$, of the internal erlge $b$; and the axis of : coincides with the axis of the cylinder. ", ", w are the displacements in the directions in which the coordinates $\rho, \omega, z$ increase.

\section{Problem 1. Symmetrical tiansierse displacement.}

The displacement $r$, in the most general case, is given by a series involving cosines and sines of multiples of $\omega$. We can determine the symmetrical term of the series. 'This "onstitutes the whole solution when the plate is subjerterl mily to symmetrical torsional force.

For a transverse force $\Omega_{1}$ applied at the point $\left(\rho_{1}, \omega_{1}, \hat{\nu}_{1}\right)$ we have seen in article 29 that the solution is:

$$
\begin{aligned}
& \psi=\frac{d \psi^{\prime}}{d \rho_{1}} \\
& \theta=-\frac{1}{\rho_{1}} d \theta_{\omega_{1}^{\prime}} \\
& \phi=-\frac{1}{\rho_{1}} \frac{d \phi^{\prime}}{d \omega_{1}}
\end{aligned} \mid \cdot \frac{\Omega_{1}}{4 \pi \mu(a+1)}
$$

The solution for a constant linear distribution of transverse fore on the circle $\rho=\rho_{1}$, $z=z_{1}$, of intensity $\Omega_{1} / 2 \pi \rho_{1}$ per unit length, is found by integrating this with respect to $\omega_{1}$ from 0 to $2 \pi$, and dividing by $2 \pi$. The result of the integration is simply to 
eliminate all but the ymmetrical part of $\psi$, and to eliminate $\theta, \phi$ altogether. The solution is therefore,

when $\rho>\rho_{1}$,

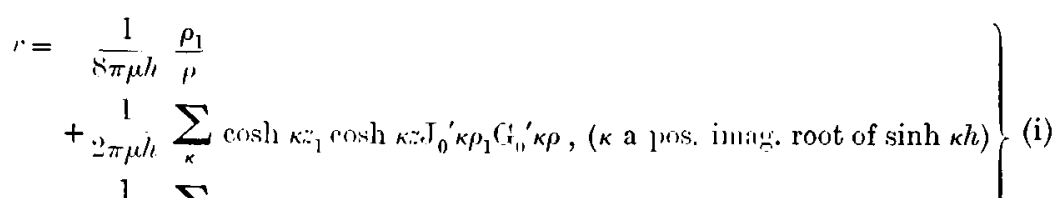

$$
\begin{aligned}
& -\frac{1}{2 \pi \mu h} \sum_{\kappa} \sinh \kappa_{\kappa:{ }_{1}} \sinh \kappa J_{0}{ }^{\prime} \kappa \rho_{1}{ }^{\prime} i_{0}{ }^{\prime} \kappa \rho,(\kappa \text { a pos. imag. root of } \cosh \kappa h)
\end{aligned}
$$

when $\rho<\rho_{1}, \rho$ and $\rho_{1}$ have simply to be interchanged.

The only stress across a cylinder $\rho$ is

$$
\hat{\rho \omega}=\mu\left(\frac{d v}{d \rho}-\frac{n}{\rho}\right) \text {. }
$$

Hence if $\Omega_{a}, \Omega_{b}$ be the transverse components of the traction on $\rho=1, \rho=b$, and $v_{1}$ the tramsrerse displacement at $\left(\rho_{1}, \omega_{1}, i_{1}\right)$ produced by this traction, Betti's Theorem gives, as in (97), for the permanent part of $v_{1}$,

$$
\frac{1}{2 \pi} \int_{0}^{2 \pi} r_{1} d \omega_{1}=\frac{1}{8 \pi \mu h} \iint\left(\Omega_{a} \frac{\rho_{1}}{c}+v_{i} \frac{2 \mu \rho_{1}}{c^{2}}\right) d \Phi_{a}+\frac{1}{8 \pi \mu h} \iint \Omega_{b} \frac{1}{\rho_{1}} d \dot{s}_{b} .
$$

Also, since the distributions $\Omega_{a}, \Omega_{b}$ have equal moments about $U_{\tau}$,

$$
a \iint \Omega_{u} d \dot{S}_{a}+b \iint \Omega_{b} d \Phi_{b}=0 .
$$

In the case of a symmetrical deformation, we have therefore

$$
v_{1}=\frac{b^{2}}{4 \mu h}\left(\frac{1}{\rho_{1}}-\frac{\rho_{1}}{u^{2}}\right) \int_{-h}^{h} \Omega_{b} d z+\frac{\rho_{1}}{a} v_{a} .
$$

The displacement $c_{1}=\rho_{1}$ being merely a rigid body rotation about $0 ;$, the permanent solution is practically

$$
v_{1}=\frac{1,2}{4 \mu h \rho_{1}} \int_{-h}^{h} \Omega_{l}, d z=-\frac{t^{2}}{4 \mu h \rho_{1}} \int_{-h}^{h} \Omega_{2, l z} \quad \text {. . . . . . . . }
$$

This might have been got at once by omitting the term $r=\rho_{1} / 8 \pi \mu h \rho$ from the source solution, in accodaure with the method explained at the end of the last article.

For a uniform system of couple about the edge-normal $\int \Omega_{b} d z$ vanishes, and the permanent displacement is rigorously null.

For the transitory part of the solution we will, to simplify the algebra, suppose the cylinder solir.

Further, this not baing a case where the separation into odd and even purts in $z$ is of much consequence, we may shorten the formule by combining the two $\kappa$ series into one.

Thus $\cosh \kappa(h+\xi) \cosh \kappa\left(h+z_{1}\right)$ being obviously equal to $\alpha$ sh $\kappa * \cosh \kappa \tau_{1}$ when 
$\sinh \kappa h=0$, but equal to $-\sinh \kappa z \sinh \kappa z_{1}$ when $\cosh \kappa h=0$, we may transform (i) if we put $h+z=\zeta, h+z_{1}=\zeta_{1}$, into

For

$$
v=\frac{1}{8 \pi \mu h} \frac{\rho_{1}}{\rho}+\frac{1}{2 \pi \mu h} \sum_{\kappa} \cosh \kappa \zeta_{1} \cosh \kappa \zeta J_{0}{ }^{\prime} \kappa \rho_{1} \mathrm{G}_{0}{ }^{\prime} \kappa \rho,(\kappa \text { a pos. imag. root of } \sinh 2 \kappa h) \text {. }
$$

$$
r=\mathrm{G}_{0}{ }^{\prime} \kappa \rho \text {, we have when } \rho=\mu, \widehat{\rho \omega}=-\frac{\mu}{a}\left(2\left(\mathrm{r}_{i,}{ }^{\prime} \kappa a+\kappa a \mathrm{G}_{0} \kappa a\right)\right. \text {. }
$$

Hence, when the transitory part of $v$ in (iii) is balanced, the solution becomes $\left(\rho>\rho_{1}\right)$

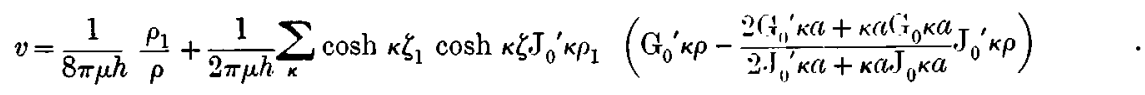

This gives at $\rho=a$,

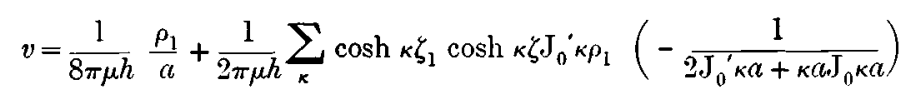

Hence, for the free displacement at $\left(\rho_{1}, z_{1}\right)$ under symmetrical transverse traction $\Omega_{a}$ on $\rho=a$, Betti's Theorem gives (omitting the rigid body rotation)

From this

$$
v_{1}=\frac{a}{\mu h} \sum_{\kappa} \mathrm{J}_{0}{ }^{\prime} \kappa \rho_{1} \cosh \kappa \zeta_{1}\left(-\frac{1}{2 \cdot \mathrm{J}^{\prime}{ }^{\prime} \kappa t+\kappa a \mathrm{~J}_{0} \kappa a}\right) \int_{0}^{2 h}{ }^{\cos s l_{1} \kappa \zeta \Omega_{a} d \zeta} .
$$

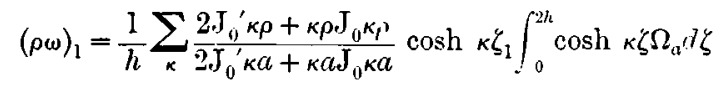

The series passes continuously, as $\rho$ increases to $a$, into the limit

$$
\frac{1}{h} \sum_{\kappa} \cosh \kappa \zeta_{1} \int_{0}^{2 h} \cosh \kappa \zeta \Omega_{a^{\prime}} l \zeta \text {, provided this latter series converges. }
$$

By Fourier's Theorem we know that it does, namely, to the value $\Omega_{a}$, it being noted that $\int_{0}^{m h} \Omega_{a} d \zeta=0$. The solution is thus verified. Of course, it could easily be obtained by the Fourier method ab initio.

The series (iv) converges very rapidly unless $\rho$ and $\rho_{1}$ are nearly equal. By an application of the Residue Calculus, it may be transformed into a series in which the functions of $\rho, \rho_{1}$ are the fluctuating functions, and the functions of $\zeta, \zeta_{1}$ the convergence factors. For consider the function of $\kappa$,

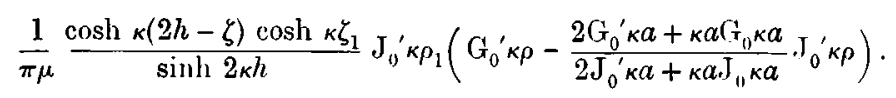

It is easy to see that $\log \kappa$ disappears from the last factor, am that the whole function is a uniform, odd function of $\kappa$; also that if $\zeta>\zeta_{1}, \rho>\rho_{1}$, the function vanishes at infinity in such a way as to make the total sum of its residues equal to zero.

The poles of the function are $\kappa=0$, the (pure imaginary) zeroes of $\sinh 2 \kappa h$, and the (real) zeroes of $2 \mathrm{~J}_{0}{ }^{\prime} \kappa r t+\kappa r \mathrm{~J}_{0} \kappa \alpha$. The function being oill, we have (series of residues at pos. imag. roots of sinh $2 \kappa h)+\left(\right.$ series of residues at pos. roots of $\left.2 \mathrm{~J}_{0}^{\prime} \kappa \kappa c+\kappa<\% \mathrm{~J}_{0} \kappa \alpha\right)$. $+\frac{1}{2}$ residue at $(\kappa=0)$, e jual to zero. 


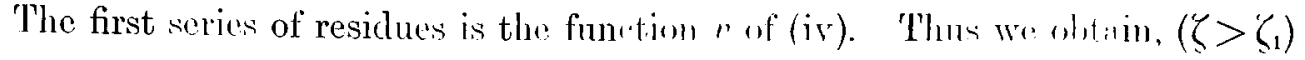

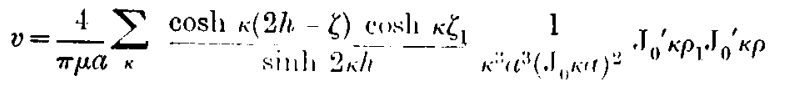

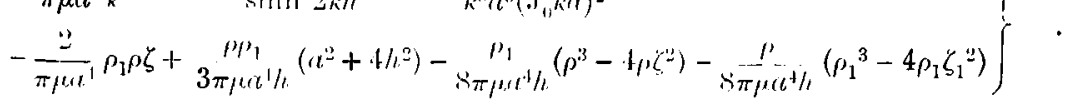

Ithen $\zeta>\zeta_{1}$, wo have merely to interehange $\zeta$ and $\zeta_{1}$ in this formula.

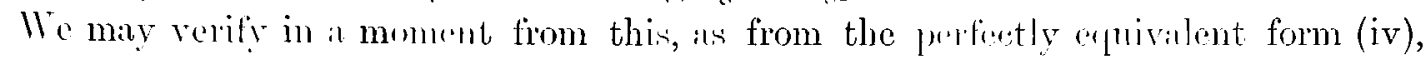
that the internal eomple is bilaneer by the stmess at the cylindrimal boumlary, and that there is mo stress alcross the plane ends. But if we remone thr last three terms from (vi), we make no change in the internal singulinity, thes trin beim the same whether sor $\zeta_{1}$ be the greater.

We thus obtain the displacement when the internal (..nuple is lalinenl at the plane ends, namely, $\left(\zeta>\check{\varsigma}_{1}\right)$

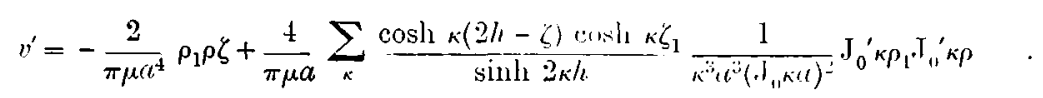

Here, as in (vi), the summation extends over the positive ronts of $2 . \mathrm{J}_{1}^{\prime} k r+\kappa a \mathrm{~J}_{10} \kappa c$. The solution for symmetric transverse traction $\Omega_{2 h}, \Omega_{0}$ on the ent $;$, which might be obtained in an abnormal form from (iv) with the comilnte formula for $\rho>\rho_{1}$, is siven in normal form hy a direct application of Betti's Theorem to (rii).

Thus

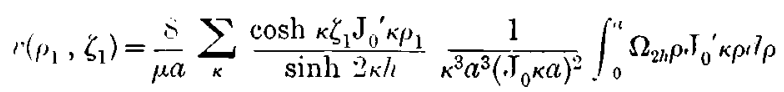

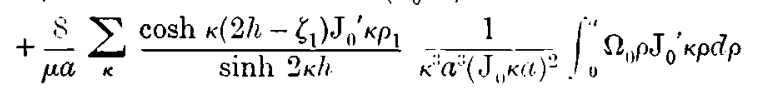

$$
\begin{aligned}
& -\frac{4}{\mu a^{4}} \rho_{1} \zeta_{1} \int_{0}^{a} \Omega_{0} \rho^{2} d \rho
\end{aligned}
$$

The result belongs rather to the theory of a long rod than to that of a thin plate. The permanent term depends only on the integral couple, and coincides with that given by SaInt Venant's theory of T'orsion.*

38. Problem 2. Boundary ralues of the normal displacencht u, and the sherriving stress normal to the plate $\widehat{\mathrm{z} \rho}$, are given functions symmetricul allout the aris; the displacement $\mathrm{v}$, or the shearing stress 0 ow, muishe's.

We begin with the case of a solid erlinder.

(i) Permanent extensional mole.

Referring to $\S 33$, I. (i), we see that under the conditions proposed the function $\mathrm{E}_{1}$ must vanish, and the solution in cylindries is

$$
\begin{aligned}
& u\left(\rho_{1}, \ddot{1}_{1}\right)=\frac{d \mathrm{E}_{2}}{d \rho_{1}}+\frac{3-a}{a+1}\left(\frac{1}{2} \cdots 1^{2}-\frac{1}{3} h^{2}\right), d \rho_{1} r_{1}{ }^{2} \mathrm{~K}_{2} \\
& w\left(\rho_{1}, \dot{1}_{1}\right)=\frac{a-3}{a+1} \ddot{1}_{1} \nabla_{1}^{2} \mathrm{E}_{2}
\end{aligned}
$$

* The writer hopes to publish shortly a solution of the problem of equilibrium of an infinite circular crlinder, in which the celebrated solutions of SAINT VENANT will :lp'pear as the leading terms. It will be shown that in a finite cylinder the permanent modes are siven exactly by SAINT Visast's theory. In the theory of thin plates, the permanent modes can only, in general, be found approximatcly.

TRANS. ROY. SOC. EDIN., VOL. XLI. PART I. (NO. 8). 
where $\quad \mathrm{E}_{2}=\frac{1}{32 \pi \mu h}$. work diffce. from $\theta=\phi=\chi(\rho)-\frac{1}{2} z^{2} \log \rho+\frac{1}{4} \rho_{1}{ }^{2} \log \rho$,

or $\quad E_{2}=\frac{\rho_{1}{ }^{2}}{128 \pi \mu h}$. work diffce. from $\theta=\phi=\log \rho$, the part omitted being merely a constant.

Now in the system $\theta=\phi=\log \rho$

Hence the solution

$$
\begin{array}{l|l}
u=(a+1) / \rho & \hat{\rho \rho}=-2 \mu(a+1) / \rho^{2} \\
w=0 & \hat{\rho}=0
\end{array}
$$

$$
\begin{array}{l|l}
u=-(\dot{a}+1) \rho / a^{2} & \hat{\rho \rho}=2 \mu(\alpha-7) / a^{2} \\
w=-2(\alpha-3) ; / a^{2} & \widehat{\rho} \tilde{z}=0
\end{array}
$$

will, taken along with $\theta=\phi=\log \rho$, give $u$ and $\widehat{\rho z}=0$ at $\rho=\alpha$. The balanced solution gives $\widehat{\rho \rho}=-16 \mu / \alpha^{2}, w=2(3-\alpha) z / \alpha^{2}$, at the edge.

Hence

and

$$
\mathbf{E}_{2}=\frac{\rho_{1}^{2}}{16 a h} \int_{-n}^{h}\left\{\frac{Z_{a}}{2 \mu}(3-\alpha) i+4 u_{a}\right\} d z
$$

$$
\left.\begin{array}{l}
u\left(\rho_{1}, z_{1}\right)=\rho_{1} \\
v\left(\rho_{1}, z_{1}\right)=2_{a+1}^{a-3}
\end{array}\right\} \cdot \frac{1}{8 a h} \int_{-h}^{h}\left\{\frac{Z_{a}}{2 \mu}(3-a) z+4 u_{a}\right\} d z^{\prime \prime}
$$

(ii) Transitory extensional modes.

The solution is given by (100) with

$$
\mathrm{E}_{4}=\frac{\mathrm{J}_{0} \kappa \rho_{1}}{8 \pi \mu(a+1) \kappa^{2} h(\cosh 2 \kappa h+1)} \text {. work diffce. from the system }\left\{\begin{array}{l}
\phi=\mathrm{G}_{0} \kappa \rho \cosh \kappa z \\
\theta=\cosh 2 \kappa h \cdot \phi
\end{array}\right.
$$

In the system mentioned

$$
\begin{array}{r}
u=\kappa \mathrm{G}_{0}{ }^{\prime} \kappa \rho\{(\cosh 2 \kappa h+a) \cosh \kappa z+2 \kappa z \sinh \kappa z\} \\
w=\kappa \mathrm{G}_{0} \kappa \rho\{(\cosh 2 \kappa h-a) \sinh \kappa z+2 \kappa: \cosh \kappa z\} \\
\frac{1}{\kappa^{2}} \frac{\tilde{z \rho}}{2 \mu}=\mathrm{G}_{0}{ }^{\prime} \kappa \rho\{(\cosh 2 \kappa h+1) \sinh \kappa z+2 \kappa z \cosh \kappa z\} \\
\frac{1}{\kappa^{2}} \frac{\hat{\rho \rho}}{2 \mu}=-\mathrm{G}_{0} \kappa \rho\{(\cosh 2 \kappa h+3) \cosh \kappa z+2 \kappa: \sinh \kappa z\} \\
-\frac{1}{\kappa \rho} \mathrm{G}_{0}{ }^{\prime} \kappa \rho\{(\cosh 2 \kappa h+\alpha) \cosh \kappa z+2 \kappa: \sinh \kappa z\} .
\end{array}
$$

The balancing system for $u$ and $\widetilde{z \rho}$ at $\rho=a$ is therefore

$$
\phi=-\frac{\mathrm{G}_{0}{ }^{\prime} \kappa a}{\mathrm{~J}_{0}{ }^{\prime} \kappa a} \mathrm{~J}_{0} \kappa \rho \cosh \kappa * i, \theta=\cosh 2 \kappa h \phi .
$$

In the balanced system, at the edge

$$
\begin{aligned}
w & =\frac{1}{a J_{0}^{\prime} \kappa a}\{(\cosh 2 \kappa h-a) \sinh \kappa z+2 \kappa z \cosh \kappa z\} \\
\frac{\rho \rho}{2 \mu} & =-\frac{\kappa}{a J_{0}^{\prime} \kappa a}\{(\cosh 2 \kappa h+3) \cosh \kappa z+2 \kappa z \sinh \kappa z\}
\end{aligned}
$$

Hence for the free solution with edge values $u_{-}=u_{a}, \widehat{z \rho}=Z_{a}$,

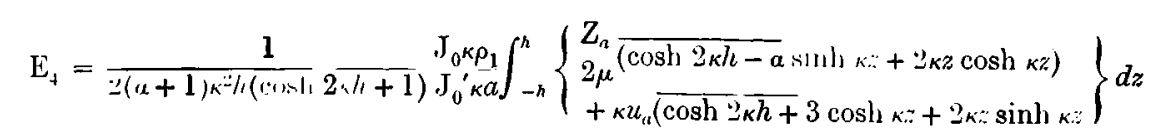


(iii) Permunent flexurul mode.

The solution is given ly (101) with

In the system

$$
\mathrm{F}_{1}=\frac{:}{32 \pi \mu h^{3}} \cdot \frac{1}{4} \rho_{1}{ }^{2} . \quad \text { work diffec. from } \quad \theta=-\phi=z \log \rho .
$$

Hence the solution balancing $"$ and $\approx$ at $\mu=x$ is

$$
\theta=-\phi=z \log \rho, \quad u=-(a+1) \cdots / \rho \mid \begin{aligned}
& \widehat{z_{p}}=0 \\
& \bar{\rho} \rho=(a+1) \log \rho
\end{aligned}
$$

$$
\begin{array}{ll}
u=(a+1): i \rho / a^{2} & \begin{array}{l}
\hat{z_{\rho}}=0 \\
w=-(a+1) \rho^{2} / 2 a^{2}+(a-3) z^{2} / a^{2}
\end{array} \\
\hat{\rho \rho \rho}=2 \mu(7-a) z / a^{2}
\end{array}
$$

In the balanced solution, at the edge

$$
w=(a+1)\left(\log a-\frac{1}{2}\right)+(a-3)::^{2} / a^{2} ; \quad \widehat{\rho \rho}=16 \mu z / a^{2}
$$

The constant term in the value of $w$ will disappear since $\int_{-h}^{h} \widehat{a p} / z=0$. Thus

and

$$
\mathrm{j}_{1}=\frac{3}{32 a h^{3}} \rho_{1}^{2} \int_{-h}^{h}\left\{\frac{Z_{u}}{2 \mu}(a-3): z^{2}-8: u u_{t}\right\} d z
$$

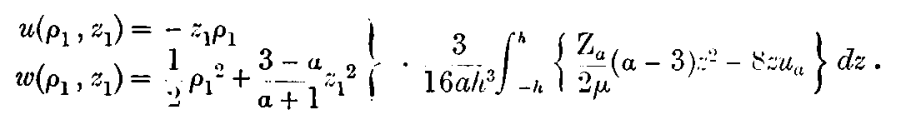

(iv) Transitory flexural modes.

The solution is given by (103) with

In this system

$$
\mathrm{F}_{3}=\frac{\mathfrak{J}_{0} \kappa \rho_{1}}{8 \pi \overline{\mu(a+1) \kappa^{2} h(\cosh 2 \kappa h-1)}} \cdot \text { work diffce. from the system }\left\{\begin{array}{l}
\phi=G_{0} \kappa \rho \sinh \kappa z \\
\theta=-\cosh 2 \kappa h \cdot \phi
\end{array}\right.
$$

$$
\begin{aligned}
& u=\kappa \mathrm{G}_{0}{ }^{\prime} \kappa \rho\left\{(a-\cosh 2 \kappa h) \sinh \kappa: z+2_{\kappa::} \cosh \kappa: z\right\} \\
& w=\kappa G_{0} \kappa \rho\{-(a+\cosh 2 \kappa h)(v) \cdot h \kappa z+2 \kappa * \sinh \kappa z\} \\
& \frac{1}{\kappa^{2}} \frac{\hat{z \rho}}{2 \mu}=G_{0}{ }^{\prime} \kappa \rho\left\{(1-\cosh 2 \kappa h) \cosh \kappa z+2 \alpha_{\kappa: *} \sinh \kappa *\right\} \\
& \frac{1}{\kappa^{2}} \frac{\widehat{\rho} \rho}{2 \mu}=G_{0} \kappa \rho\{(\cosh 2 \kappa l \ell-3) \sinh \kappa:=2 \kappa: \cosh \kappa=\} \\
& -\frac{1}{\kappa \rho} \mathrm{G}_{0}{ }^{\prime} \kappa \rho\{(a-\cosh 2 \kappa \lambda) \sinh \kappa=+2 \kappa z \cosh \kappa=\}
\end{aligned}
$$

The system balancing $u$ and $\widehat{z \rho}$ at $\rho=\alpha$ is

$$
\phi=-\frac{\mathrm{G}_{0}{ }^{\prime} \kappa a}{J_{0}^{\prime} \kappa a} \mathrm{~J}_{0} \kappa \rho \sinh \kappa:, \theta=-\cosh 2_{\kappa} h \cdot \phi .
$$

In the balanced system, at the edge

$$
\begin{aligned}
& w=\frac{1}{a \mathrm{~J}_{0}^{\prime} \kappa a}\left\{-(a+\cosh 2 \kappa h) \cosh \kappa *+2 \kappa_{*} \sinh \kappa * ;\right\}
\end{aligned}
$$

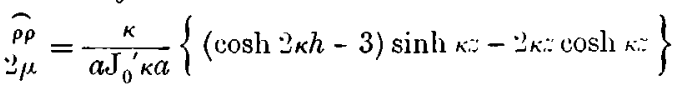

Hence for the free solution with erlge values $u=u_{a}, \widehat{\tilde{\rho}}=Z_{t}$,

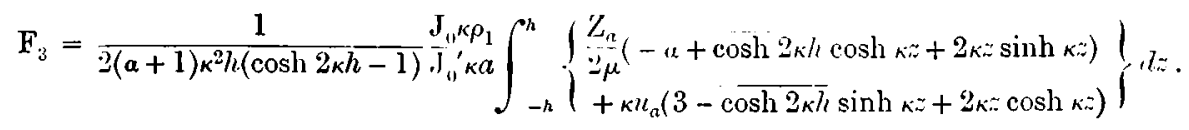

If the given values of $Z_{a}, u_{a}$ are the same as the edge values of $\widehat{z p}, w$, in one of the particular solutions, then clearly this particular solution by itself is the solution, and the 
integrals which define the coefficients of the other particular solutions must vanish, while the integral corresponding to the solution left has its value determined. These results are easily verified by actual integration.

This remark may be userl to find the solution for a hollow cylinder, which of course might also be obtained directly by the above process. We shall illustrate the method by finding the value of $F_{3}$ corresponding to any given root $\kappa$ of $\sinh 2 \kappa h-2 \kappa h$, when we have given $u_{a}, \|_{b}, Z_{a}, Z_{b}$.

This value of $\mathrm{F}_{3}$ we know is of the form $\mathrm{AJ}_{0} \kappa \rho_{1}+\mathrm{BG}_{0} \kappa \rho_{1}$.

The complete values of $\|$ and of $\approx \rho_{i}-\omega_{\mu}$ for $\beta=\rho_{1}$ are given by series which manifestly converge uniformly sor long 4 s $b<\rho_{1}<a$.

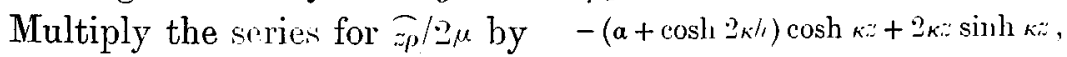

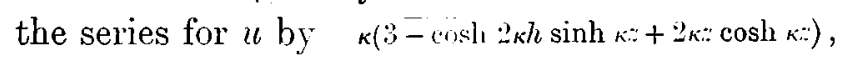

add, and integrate with respect to $z$ from $-h$ to $h$. All the terms disappear except that associated with the given root $\kappa$. We thus find

$$
\begin{aligned}
& \left(A J_{0}{ }^{\prime} \kappa \rho_{1}+\mathrm{BG}_{0}{ }^{\prime} \kappa \rho_{1}\right) 2(a+1) \kappa^{2} h\left(\cosh 2{ }^{\prime} \kappa h-1\right)
\end{aligned}
$$

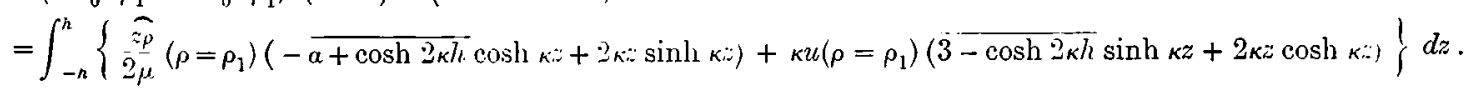

This is proved for the case $l<\rho_{1}<\|$. Now take the limits of both sides for $\rho_{1}=a$. The limit of the integral is found simply by replaring $z \rho$ and $u\left(\rho=\rho_{1}\right)$ in the integrand by $\widetilde{z \rho}$ and $u(\rho=a)$, promidnd the resulting integral has " meaning, which will be the case if $\widetilde{z \rho}$ and $u(\rho=\prime \prime)$ are integrable functions of $z$. Similarly we may take the limit for $\rho_{1}=b$, and thus obtain two equations to determine A and B.

It will be observed that by this method we avoid two difficulties which in problems of this kind are often introrluced unnecessarily by physical writers, namely, (i) the difficulty as to the convergence of the series for $\widetilde{z \rho}$ and $u$, when the value $\rho_{1}=a$ or $b$ is substituted term by term, and (ii) the allied difficulty as to the continuity of the series right up to $\rho=a$ or $b$, even when it is known to converge. Judging from analogy, we maty feel reasonably certain that the serics will in fact converge at the limits, at least in the rase of ordinary functions; but it is worth while noting that, whether they converge or not, the Fourier method of assuming the continuity and convergence, and determining the coctficients by integration, does wive the correct values of these coefficients.

On the other hand, while our 'Green's function' method proves definitely that any possible solution has the form siven above, it does not prove that a solution is possible for arbitrary edge values of and $"$. The investigation might be completed by verifying that the solution obtained does actually satisfy the conditions, which would not be difficult in the present case. Alternatively, we may rely upon physical considerations, or upon a general allalytical existence theorm. The proofs of theorems of this type in other binches of physical mathematics haw buen consid maly improved within recent years by Poincarr and others, and their methods alc cyually applicable to the elastice equations. 
39. Problem 3. To determine the premusent modes, leacing given the

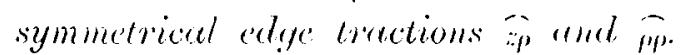

Supposing the cylinder solid, we have only to make as slight molification in the pricess of (i), (iii) in last article.

Firtensional mode.

In (i) the balaneing solution must now be taken as

$$
u=\frac{(a+1)^{2}}{i-a} \frac{\rho}{u^{2}}, u=-\begin{gathered}
2(3-a)(u+1) \\
7-u
\end{gathered} u^{2} .
$$

This, aloug with $\theta=\phi=\operatorname{lng} \rho$, wives $\widehat{\rho}$ and $\hat{\rho}=0$ at $\rho=r$.

The balanced solution gives at the elge

Hence

$$
u=\frac{8 a+1}{u i-u} \cdot u=-\frac{2(3-a)(a+1)}{i-a} \| .
$$

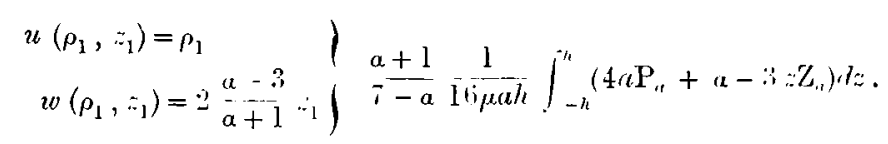

This gives the permanent mode exactly. The ordinary approximate theory omits the term in $Z_{a}$ from the integral.

- In CHrEF'i solution of the problem of a rotating disc, the stress 20 vanishes identically at the alge, while $\int_{-h}^{h} \mathrm{P}_{n}{ }^{\prime}=0$. His solution is therefore exact, so far as the fundamental mode is concerned.

As in last article, we infer from the form of the ahore solution that for any other than the permanent mode $\quad \int_{-h}^{h}(t \rho \hat{\rho \rho}+a-3: \hat{\rho})=0$.

This may be verified by actual integration. Further, in the case of a hollow cyliniler the solution is of the form

$$
\left.\begin{array}{l}
u\left(\rho_{1}, \ddot{1}_{1}\right)=A \rho_{1}+B \mu_{1} \\
u\left(\rho_{1}, \ddot{1}_{1}\right)=2 A(a-3) \cdot \ddot{\prime}_{1},(a+1)
\end{array}\right\}
$$

and the coefficints $A, B$ are found from the conditions that

$$
\int_{-h}^{h}(4 \rho \cdot \hat{\rho}+\bar{a}-3: \because \cdot \hat{z \rho}) d z \text { must, for } \rho=a \text { and } \rho=b
$$

have the same value for the assumed form and for the given tractions.

Flexural morle

Referring to (iii) of last article, the solution balincing

$$
\begin{aligned}
& \theta=\phi=\approx \log \rho \text { at } \rho=a \text { is now } \\
& u=-\frac{(a+1)^{2}}{7-\alpha} \frac{n \rho}{a^{2}} ; w=\frac{(a+1)^{2}}{7-a} \frac{p^{2}}{2\left(t^{2}\right.}+\begin{array}{c}
(3-a)(u+1) \\
\tau-\alpha
\end{array} \frac{z^{2}}{t^{2}} .
\end{aligned}
$$

The balanced solution gives at the calye

$$
u=-8 \quad a+\frac{1}{7-a} \frac{z}{a} ; w=\text { const }+\frac{(3-a)(a+1)}{7-a} i^{\prime \prime} .
$$


Hence

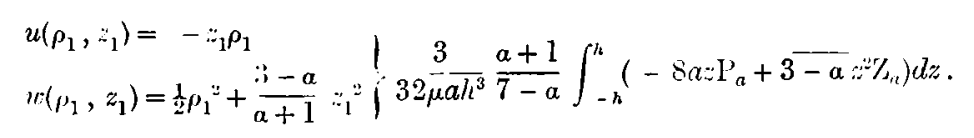

The ordinary approximate theory takes account of the term in $z \mathrm{P}_{a}$ only.

The solution for a hollow cylinder can be obtained by this method, or by taking, in the notation of (94),

$$
\mathrm{F}=\mathrm{A} \rho_{1}{ }^{2}+\mathrm{B} \log \rho_{1}+\mathrm{C}\left(\frac{1}{4} \rho_{1}{ }^{2} \log \rho_{1}-\frac{1}{4} \rho_{1}{ }^{2}\right),
$$

determining $\mathrm{C}$ from the value of $\int_{-h}^{h} \widehat{z \rho} d z$ at either edge, and then determining $A$ and $B$ from the conditions that

$$
\int_{-h}^{n}(-8 \rho \tilde{\rho} \bar{\rho}+\overline{3}-\bar{a}: \because \bar{p}) d z \text { must for } \rho=a \text { or } \rho=b
$$

have the same value in the assumed form as in the actual displacement.

\section{Eixpensions of arbitrary functions.}

When we attempt to apply the method of last article to the determination of the modes corresponding to the various roots of $\sinh : \kappa h \pm * k h$, we are at once confronted with an apparently insuperable difficulty. The determination of any one mode is reduced by the application of Betti's Theorem to the special problem of balancing the particular source solution involving a given root $\kappa$. Now in similar investigations connected with Laplace's equation, the equation of conduction of heat, and other partial differential equations of the second order which occur in physical mathematics, the analogous balancing problem can be solved without difficulty for certain simple forms of edge, and the balancing solution is of the same type as the particular source solution, that is, involves only the same root $\kappa$. In the present problem, however, the balancing solution will in general involve particular solutions of all types, as will be seen below.

Various theorems relating to the expansion of arbitrary functions may be found, similar to the theorem suggester at the cml of $\S 38$, but these do not help us, at all events immediately, to the weneral solution sought. One way of obtaining these expansion theorems may be indicated here; the methor is of very wide application.

On a circular cylinder $\rho=\alpha$, within the infinite plate, let areal force be distributed, the components of its intensity per unit area being $\mathrm{P} \cdots s m \omega, \Omega \sin m \omega, \mathrm{Z} \cos m \omega$, where $\mathrm{P}, \Omega, \mathrm{Z}$ are functions of $z$.

The infinite plate solution for this distribution of force can br written down, and the components of stress $\widehat{\rho \rho}, \widehat{\rho \omega}, \hat{\rho z}$, calculated. These are given in different analytical forms according as $\rho$ is greater or less than " . The expansion theorems are derived from the conditions of equilibrium

$$
\underset{\epsilon=0}{\mathrm{~L} t}\{\widehat{\rho \rho}(\rho=\|-\epsilon)-\widehat{\rho \rho}(\rho=\|+\epsilon)\}=P
$$

with two similar equations for $\Omega, \mathrm{Z}$. 
There are other axpmusions which we know must exist, lout the ecefficients of which we cammot determine. The following rxamples are of special importance, and will be found useful immerliately. They refer to the asse of it plate bounded by a single infinite arlge, filling, sily, the region $r>0$, and the displarements considered are such that $u$, $"$ are fum tions of $r,:$ only, while $"$ vanishes.

Extensional mudes.

The permanent mole is of the form $u=x, u^{\prime \prime}=\begin{gathered}x-3 \\ a+1\end{gathered}:$.

In a transitory morle with $\phi=e^{i \kappa * r} \cosh \kappa z, \theta=\cosh 2 \kappa h \cdot \phi$, we have

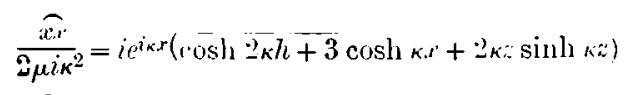

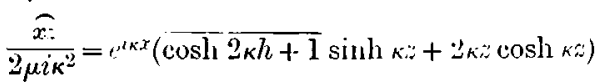

As a special case of the results of last article, it follows that the coefficient of the permanent mole is determined from the given value of $\int_{-h}^{h} d:$ at the orlge; and this integral normal stress is zero for wich of the transitory modes.

Hence if $\mathrm{P}(z)$ be any even function of $z$, with $\int_{-h}^{h} \mathrm{I}(z) d z=0$, and $\mathrm{Z}(z)$ be any old function of $z$, coefficients $\mathrm{C}_{\kappa}$ exist such that at the same time

and

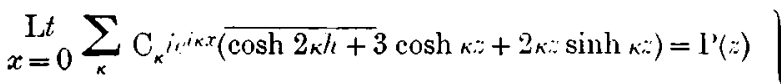

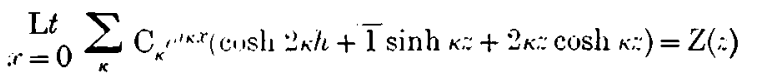

Flexural morl's.

In the permanent mode $\mathrm{F}$ of equation (95) is of the form $\mathrm{A} x^{2}+\mathrm{B} x^{3} . \mathrm{A}$ and $\mathrm{B}$ are found from the edge values of $\left.\int_{-h}^{h} z \cdot x\right)$ and $\int_{-h}^{h} \hat{x} d x$. These integrals vanish for a transitory mote, in which, with $\phi=e^{i \kappa x} \sinh \kappa z, \theta=-\cosh 2 \kappa h \cdot \phi$,

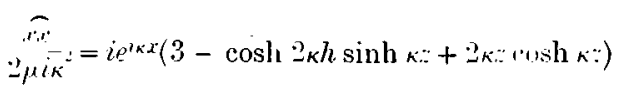

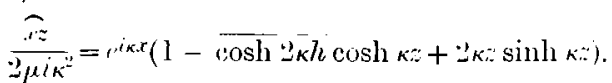

We infer that if $P(z)$ be any odd function of $z$, with $\int_{-n}^{n} z \mathrm{P}^{\prime}(\xi) d z=0$, and $Z(z)$ any even function of $z$, with $\int_{-n}^{h} Z(:) d z=0$, values of $C_{x}$ exist such that simultaneously

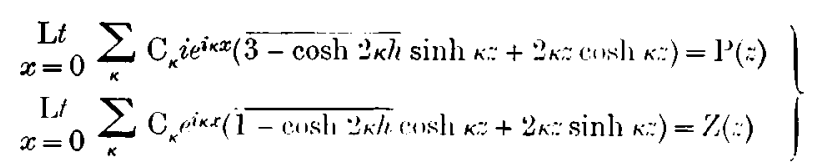

The limit for $x=0$ may be tikien term by term, provided the resulting series converge. In the following analysis we shall assume that they do so, but this is merely in order to avoid lengthy forms of statement; the argument could be put, if necessary, in a form independent of this assumption. 
41. The problem of given edge tractions for a thin plate.

The form of the complete solution is exactly known, and the three boundary conditions in their exact forms could, therefore, at once be written down. The whole strain is compounded of an infinite number of morles of equilibrium of known types, and it is obviously suggested as the method of attack that we should try to disentangle from the general boundary conditions those special conditions by which each mode is separately defined. When the plate is thin we find that within certain limits this can be done, and, in particular, the conditions defining the permanent modes, which in the case supposed are incomparably the most important, can be found with considerable exactness.

We shall understand that the edge traction, or any component of the edge traction, is given as a function of $x, y, z / h$ or of $s, z / h$, where $s$ is the arc of the edge line, so that if $\zeta$ be put for $z / h$ the form of this function is completely independent of $h$. The theory may be applied to cases in which the proviso is not fulfilled, but before such application the given traction is to be separated into pints of ascending order in $h$, say, for example, $f_{0}(x, y . \zeta)+h f_{1}(x, y, \zeta)+h^{2} f_{0}(x, y, \zeta)+$ etc. ; then for a first approximation we deal only with $f_{0}(x, y, \zeta)$. The theory does not contemplate such a distribution of traction, as, for example, $\sin (m s / h), m$ being a number, where the rate at which the traction varies along the are is of a lower order in $h$ than the traction itself.

The trace of the cylindrical edge on the middle plane of the plate is the edge line; the outward normal, and the tangent, to the edge line will be referred to as the normal, and tangent simply; the generator of the cylindrical edge at right angles to these at their point of intersection may be called the perpendicular.

Let $l, m$ be the direction cosines of the normal, then $-m, l$ are those of the tangent.

The normal displacement is $\quad p=l u+m v$ and the tangential displacement $q=-m u+l v$.

The tractions on the edge in the directions of normal, tangent, and perpendicular, are $\widehat{n n}, \widehat{n s}, \widehat{n z}$ or $\mathrm{N}, \mathrm{S}, \mathrm{Z}$.

\section{Extensional strain.}

In this case $\mathrm{N}, \mathrm{S}$ are even functions, ancl $\mathrm{Z}$ : in odd function of $*$

It will be advantageous to express as fill as possible the displacements anıl tractions at an edge in the various types of solution in terms of lerivatives along the tangent and normal.

Alongside the symbol $\alpha$ we shall use the more familiar $\sigma$, the relation between the two being given by $a+1=4(1-\sigma) ; 3-a=4 \sigma$. 
(i) Permanent morle.

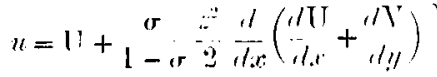

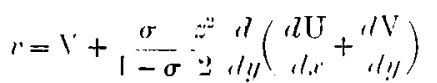

$$
\begin{aligned}
& w=-\frac{\sigma}{1-\sigma}\left(\frac{d \mathrm{U}}{d \gamma_{i}}+\frac{d \mathrm{~V}}{d i j}\right)
\end{aligned}
$$

where

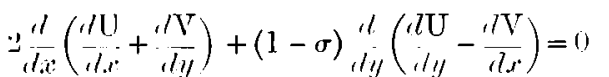

$$
\begin{aligned}
& 2 \frac{d l}{d ! !}\left(\frac{d \mathrm{U}}{d, x}+\frac{d \mathrm{~V}}{d ! y}\right)-(1-\sigma) \frac{d \prime}{d x}\left(\frac{d \mathrm{U}}{d ! !}-\frac{d \mathrm{~V}}{d \cdot t}\right)=0
\end{aligned}
$$

Put

$$
\begin{aligned}
& \left.\Delta=\frac{1}{1-\sigma}\left(\frac{d \mathrm{~L}^{5}}{d x^{2}}+\frac{d v}{d y}\right)=\frac{1}{1-\sigma}\left(\frac{d u}{d, v}+\frac{d v^{\prime}}{d y}\right)\right) \\
& \Pi=\frac{l}{2}\left(\frac{d \mathrm{U}}{d ! \prime}-\frac{d \mathrm{~V}}{d x}\right) \quad=\frac{1}{2}\left(\frac{d u}{d ! \prime}-\frac{d x}{d x}\right)
\end{aligned}
$$

Then

$$
\left.\begin{array}{ll}
\frac{d \Delta}{d \cdot c}+\frac{d \Pi}{d y}=0 \\
\frac{d \Delta}{d ! \prime}-\frac{d \Pi}{d,}=0
\end{array} \mid \text { and at an e.lge } \begin{array}{l}
\frac{d \Delta}{d n}+\frac{d \Pi}{d s}=0 \\
\frac{d \Delta}{d s}-\frac{d \Pi}{d n}=0
\end{array}\right\}
$$

Also

$$
\left.\begin{array}{l}
\hat{y}=\frac{2 \mu}{1-\sigma}\left(\frac{d u}{d x}+\sigma \frac{d u}{d y}\right) \\
\hat{y y}=\frac{-\mu}{1-\sigma}\left(\sigma \frac{d u}{d x}+\frac{d v}{d y}\right) \\
\hat{x}=\frac{2 \mu}{1-\sigma} \cdot \frac{1-\sigma}{2}\left(\frac{d u}{d y}+\frac{d y}{d x}\right)
\end{array}\right)
$$

The components of traction parallel to the axes are

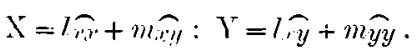

These are easily transformer into

$$
\mathrm{X}=2 \mu\left(l \Delta+m \Pi-\frac{d b}{d !}\right) ; \mathrm{Y}^{\prime}=2^{2} \mu\left(-l \Pi+m \nu+\frac{d u}{d s}\right) .
$$

Hence

also

$$
\left.\begin{array}{l}
\widehat{m n}=l \mathrm{X}+m \mathrm{Y}=2 \mu\left(\Delta+m \frac{d u}{d s}-l \frac{d l}{d s}\right) \\
\widehat{n s}=-m \mathrm{X}+l \mathrm{Y}=2 \mu\left(-\Pi+l^{\prime l u}+m \frac{d v}{d s}\right) \\
\widehat{n z}=0
\end{array}\right\}
$$

or

$$
\left.\begin{array}{l}
\widehat{n n}=2 \mu\left(\Delta-\frac{d y}{7 s}-\frac{\nu}{\rho}\right) \\
\widehat{n=s}=2 \mu\left(-\Pi+\frac{d p}{d \cdot s}-\frac{q}{\rho}\right)
\end{array}\right\}
$$

TRANS. ROY. SOC. EDIN., VOL. XLI. PART I. (№ 8). 
If $P . q$ be the values of $p, q$ when $z=0$, then

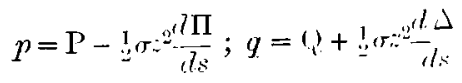

$$
\begin{aligned}
& \left.\frac{\partial \widehat{m}}{2 \mu}=\Delta-\frac{d \mathrm{Q}}{d s}-\frac{\mathrm{P}}{\rho}-\frac{1}{2} \sigma v^{2}\left(d^{2} \frac{\Delta}{d \xi^{*}}-\frac{1}{\rho} \frac{d \Pi}{d *}\right)\right)
\end{aligned}
$$

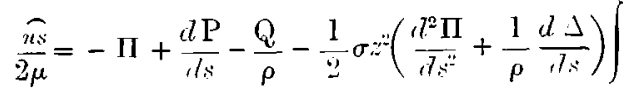

(ii) Rotational Transitory Medes.

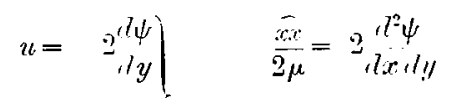

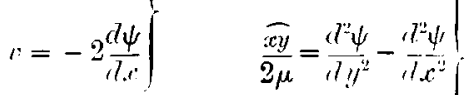

$$
\begin{aligned}
& \frac{\overline{x_{\tilde{x}}}}{\overline{2 \mu}}=\frac{d^{2} \psi}{d y d}
\end{aligned}
$$

Taking the axes of $x$ and $y$ for a moment along the normal and tangent, these give at once by means of $(k)$

$$
\begin{aligned}
& \left.p=2^{d} d s\right) \quad \frac{m n}{2 \mu}=2 \frac{d}{d s} \frac{d \psi}{d n}-\frac{2}{\rho} \frac{d \psi}{d s} \\
& \left.q=-2 \frac{d \psi}{d n}\right\} \quad \frac{\widehat{n s}}{2 \mu}=\frac{d^{2} \psi}{d z^{2}}+\frac{2}{\rho} \frac{d \psi}{d n}+2 \frac{d l^{2} \psi}{d s^{2}} ! \\
& \frac{\widehat{n}}{2 \mu}=\frac{d^{2} \psi}{d s i z}
\end{aligned}
$$

The function $\psi$ can be expressed as a series of terms of the form

$$
\psi_{n}(x, y) \cos \frac{n \pi z}{h}, \text { where } \nabla^{2} \psi_{n}-\frac{n^{2} \pi^{2}}{h^{2}} \psi_{n}=0
$$

Hence in cases where the values of $\psi$ along an edge are given independently of $h$, or generally, when the rate of variation of $\psi$ along an edge is of the same order in $h$ as $\psi$ itself, say order zero, terms of various orders occur in the expressions for the displacements and tractions.

Thus

$$
\begin{aligned}
& \frac{d \psi}{d l s}, \frac{d^{2} \psi}{d s^{2}} \quad \text { are of order } \quad 0 \\
& \frac{d \psi}{d n}, \frac{d}{d s} d \psi, \frac{d}{d n}, \frac{d \psi}{d s} \cdot \frac{}{d z} \text {. . . . . . - } 1 \\
& \begin{array}{llll}
d^{2} \psi & \text { is } \quad . \quad . \quad & -2 \\
i z^{2} &
\end{array}
\end{aligned}
$$

It follows that in such cases this type of strain contributes mainly to the tangential displacement and traction at an eige. 
We see also that the principal part of the displicement is of one order higher in $h$ than the principal part of the traction.

(iii) Dilatetional trensitony mudes.

There would bo some ulvantion in working with the functional symbols $\theta, \phi$, as with $\psi$ in the list case, but on the whole: it seems clearer to deal with a typical solution corresponding to a silugle root $\alpha$ of $\sinh : * k+\nu_{k} k$.

$$
\begin{aligned}
& \phi=\cosh \kappa: f(x, y) ; \theta=\cosh 2 \kappa h \cdot \phi
\end{aligned}
$$

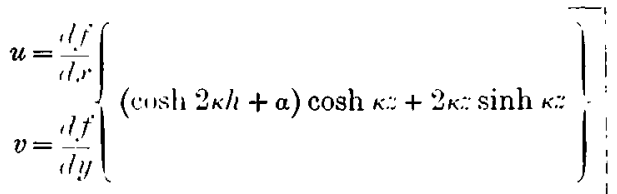

$$
\begin{aligned}
& w=\kappa f\left\{(\cosh \Xi \kappa h-a) \sinh \kappa::+\otimes_{\kappa::} \cosh \kappa::\right\}
\end{aligned}
$$

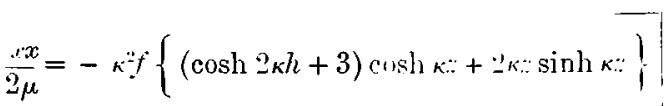

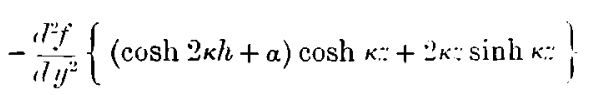

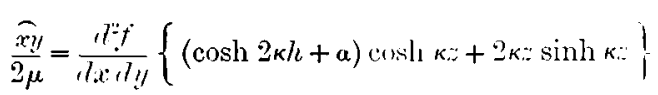

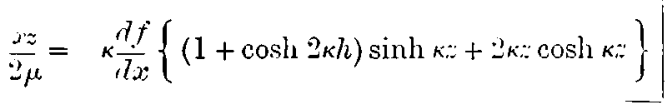

Hence

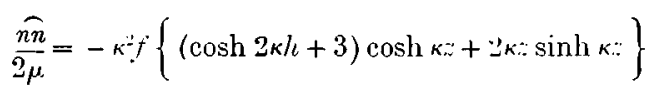

$$
\begin{aligned}
& -\left(\frac{1}{\rho} \frac{d f^{\prime}}{d h}+\frac{d^{2} f}{d \xi^{2}}\right)\{(\cosh 2 \kappa h+\alpha) \cosh \kappa=+2 \kappa * \sinh \kappa=\xi\} \\
& \widehat{\frac{n s}{2 \mu}}=\left(\frac{l}{l s} \frac{d f}{d n}-\frac{1}{\rho} \frac{d f}{l s}\right)\{(\cosh 2 \kappa h+\alpha) \cosh \kappa:=2 \kappa: \sinh \kappa: z)
\end{aligned}
$$

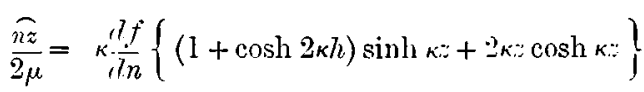

Thus at an edge where the rate of variation of $f^{\prime}$ is of the same order in $h$ as $f$ itself, say order zero, the normel and perpendicular displacements are of order -1 , while the tangential displacement is of an order one higher; the normal and perpendicular tractions are of order -2 , the tangential traction being of order -1 , or again one higher.

Hence this type of strain contributes most to those components of displacement and traction to which the $\psi$ type contributes least, at in elge.

(iv) It is now possible to assign approximately to each of the three types of strain the portion which it carries of any given distribution of edge traction. Let this distribution be N, S, Z, functions of $z$, s, of order zero in $h$. We can sitisfy the conditions to the first order by a solution in which the principal part of the traction 
is of order zero for each type of strain. For, taking account only of these principal parts, the equations to be satisfied at the edge are on this supposition

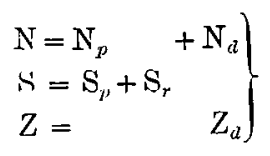

the suffixes referring to the permanent, rotational, and dilatational types respectively. Now

Also

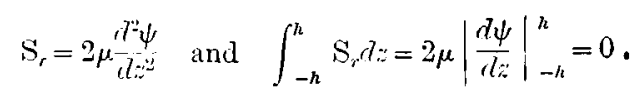

$$
\left.\mathrm{N}_{d}=-2 \mu \sum_{\kappa} \kappa^{2} \cdot f_{\kappa} \quad\{(\cosh 2 \kappa h+3) \cosh \kappa=2 \kappa * \sinh \kappa z)\right\},
$$

and, as in art. 40 ,

Hence the above equations give

$$
\int_{-h}^{h} \mathrm{~N}_{a} d z=0
$$

$$
\left.\begin{array}{l}
\mathrm{N}_{\mu}=\frac{1}{2 h} \int_{-h}^{h} \mathrm{~N} d z \\
\mathrm{~S}_{l^{\prime}}=\frac{1}{2 h} \int_{-h}^{h} \mathrm{~S} d z
\end{array}\right\}
$$

and these conditions determine the permanent mode.

$\psi$ can now be found from the boundary condition

For, taking

$$
2 \mu \frac{d l^{\prime \prime} \psi}{d l^{\prime \prime}}=S-\frac{1}{2 h} \int_{-h}^{h} \mathrm{~S} d z
$$

the condition is

$$
\psi=\sum_{n=1} \psi_{n}(x, y) \cos \frac{n \pi z}{h},
$$

$$
\sum_{n=1} \psi_{n} \cdot n^{2} \cos \frac{n \pi z}{h}=-\frac{h^{2}}{2 \mu \pi^{2}}\left\{S-\frac{1}{2 h} \int_{-h}^{h} \mathrm{~S} d z\right\} .
$$

Now the right-hand member here is a function of $s, z$, even in $z$, the $z$-integral of which from $-h$ to $h$ is zero for all values of $s$. It can therefore be expanded by Fourier's Theorem in the form, valid from $z=-h$ to $z=h$,

$$
\sum_{n=1} \mathrm{~A}_{n} \cos \frac{n \pi z}{h} \text {. }
$$

$\psi_{n}$ is then determined as satisfying $\Gamma^{2} \psi_{n}+\frac{n^{2} \pi^{2}}{l^{2}} \psi_{n}=0$ throughout the plate, and taking the value $\mathrm{A}_{n} / \mu^{2}$ at the boundiury.

Lastly, the equations to determine the dilatational mode are (since at the edge $\frac{d f}{d n}=-i \kappa f$ to the first order),

$$
\begin{aligned}
& \sum_{\kappa} f_{\kappa} \cdot \kappa^{z}\{(\cosh 2 \kappa h+3) \cosh \kappa z+2 \kappa z \sinh \kappa z\}=-\frac{1}{2 \mu}\left\{\lambda-\frac{1}{2 h} \int_{-h}^{h} \mathrm{~N} d z\right\} \\
& \sum_{\kappa} f_{\kappa} \cdot i \kappa^{2}\{(1+\cosh 2 \kappa h) \sinh \kappa z+2 \kappa z \cosh \kappa z\}=-\frac{1}{2 \mu} Z
\end{aligned}
$$


By the sime methen as in the ratse of $\psi$, but using the theorem (i) of $\$ 40$,

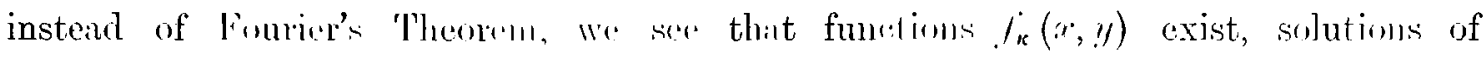

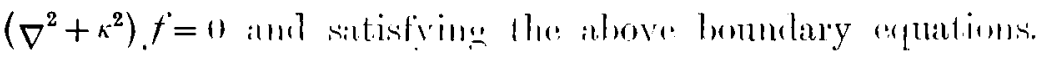

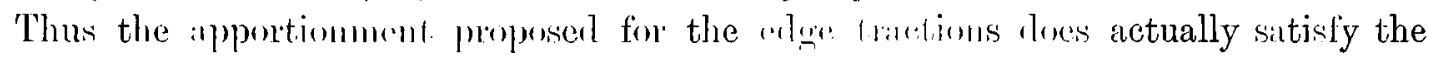
comblitions to a first approximation. Tlare solution limm gives tractions of which the principal parts are the lasetions antually assigned in the problem. The resilual traction wiven by the solution is of the lirst and higher orters; and a second approximation to the problem will be abtaines by sulutwatiug a solution giving the residual thations of the fins onder, suld solution hoing found by the methorl used in the first ipproximntion.

This proness would be tenlions, and the way would be blockend at an early stage by our ignorance of the coefficients of the expansions (i) of $\$ 40$.

We therefore pass at mue to the comsiclation of the powerful method fumished by Betti's Theorem for the determination of the permanent mode.

43. Extensionul strain. The Grech's Function method for the permanent mode.

The method has already heon explained (\$36). If we wish the permanent displacement at $\left(x^{\prime}, y^{\prime}, z^{\prime}\right)$ in any liretion (sily the displicement 1$)$, we tilis the permanent part of the solution for a unit force in that direction (a unit $\mathrm{X}$ foree). modify it by removing the terms which convey resultant stress, and then try to balunce it at the edge by adding a solution, without internal singularity, which shall neutralise its edge tractions.

The displacements at the elge in the bilinced solution, i.e. in the solution obtained as the sum of the source and balancing solutions, being $u^{\prime}, v^{\prime}, u^{\prime}$, or $p^{\prime}, q^{\prime}, w^{\prime}$, and the given tractions $\mathrm{X}, \mathrm{Y}, \mathrm{Z}$, or $\mathrm{N}, S, Z$, we have

$$
\begin{aligned}
& \left.u x^{\prime}, y^{\prime}, z^{\prime}\right)=\iint\left(\mathrm{X} u^{\prime}+\mathrm{J}^{\prime} r^{\prime}+Z m^{\prime}\right) \cdot 7 s, l: \\
& =\iint\left(\mathrm{N} p^{\prime}+\mathrm{S}^{\prime} t^{\prime}+Z \mathrm{Z} v^{\prime}\right) d s d z
\end{aligned}
$$

tle integral being taken over the cylinilrical boundary.

'The thickness $\because h$ being supposed infinitesimal, the object of the method is to determine in few of the terms of $p^{\prime}, q^{\prime}, w^{\prime}$ of lowest order in $h$.

An alternitive methol would be to determine the functions $\mathrm{E}_{1}, \mathrm{E}_{2}$ of $\$ 33$, I. (i), in trims of elge tractions; but the least confusing method of all is perhaps to detulnime

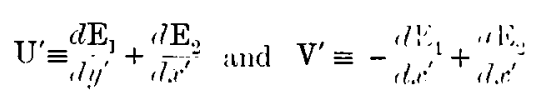

These do not contain $z^{\prime}$, but when they are known the complete solution can obviously be written down. We begin with $\mathrm{U}^{\prime}$, and in fact it will not be necessary to determine $\mathrm{V}^{\prime}$ separately. 
Now $\mathrm{U}^{\prime}$ is the work difference from the system

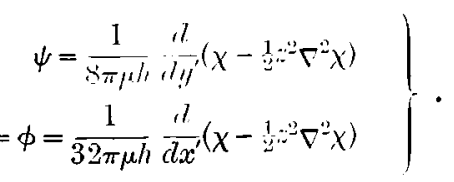

From this system let those terms be removed which transmit the resultant stress (equivalent to a unit $\mathrm{X}$ force through $x^{\prime}, y^{\prime}, z^{\prime}$ ). Then the remaining displacements have still the forms discussed in $\$ 42$ (i), and we shall use for them, and for the various quantities related to them, the notation given there, modified by the addition of a suffix 0 in each case.

The problem is now to balance the edge tractions due to the system $u_{11}, v_{0}, w_{n}$.

The principal parts of these tractions, which in this case are simply the terms independent of $z$, are balanced by a solution of the permanent type (which we shall distinguish by the suffix 1) such that at the edge

$$
\left.\begin{array}{l}
\left(\Delta_{i 1}-\frac{d Q^{\prime \prime}}{d s}-\frac{P_{11}}{\rho}\right)+\left(\Delta_{1}-\frac{d Q_{1}}{d s}-\frac{P_{1}}{\rho}\right)=0 \\
\left(-\Pi_{0}+\frac{d P_{01}}{d s}-\frac{Q_{0}}{\rho}\right)+\left(-\Pi_{1}+\frac{d P_{1}}{d s}-\frac{Q_{1}}{\rho}\right)=0
\end{array}\right\}
$$

These conditions define the solution with suffix 1 .

The residual tractions from the compound solution $u_{0}+u_{1}, v_{0}+v_{1}, w_{0}+w_{1}$ contain the factor $z^{2}$ and are of order $h^{2}$ as compared with those already balanced. To balance these residual tractions, solutions of all types are required, but, is in $\S 42$ (iv), the permanent solution (which will be marked with suttix 2 ) is determinod from the integral residual tractions; the permanent displacements are of order $h$, while those from the transitory solutions are of order $h^{2}$, those from the source solution being of order $h^{-1}$.

The displacements of the balanced solution are therefore to terms of order $h$ inclusive in the notation of $\$ 42$ (i),

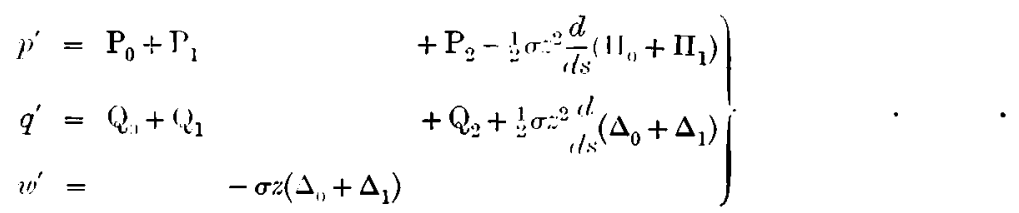

and with these values of $p^{\prime}, q^{\prime}, 1 \prime^{\prime}$

$$
\mathrm{U}^{\prime}\left(x^{\prime}, y^{\prime}, z^{\prime}\right)=\iint\left(\mathrm{N} y^{\prime}+\mathrm{S} q^{\prime}+Z u^{\prime}\right) l^{\prime} d z \quad \text {. . . . . . . }
$$

All the steps of the above process can actually be amied out in the cisc of a circular plate, and the final formula gives a perfectly refinite solution provided merely that $\mathrm{N}, \mathrm{s}, \mathrm{Z}$ are functions integrable over the erlge. It should be specially noter that, in this form of the solution, riscontinuity of the applied traction gives rise to no difticulty whatever.

On the other hand, the formuli does not give is ready answer to such an important question as "What are the rolations between the tractions actually applied, and the 
tractions refuired to maintain lhe pemanent whlution alome" or the practically equivilent question, "What conditions must the colow stress satisfy in order that the

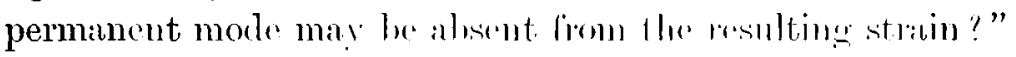

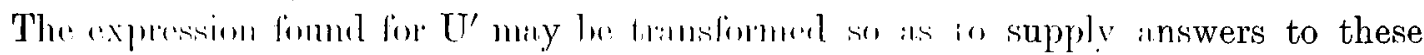
questions:

In the first plane, we mote that the values of $f^{\prime}, q^{\prime}, w^{\prime}$ will still be contere to the order stated (but will contain superfluons terms) if in the "xpressions just given for them we wite

and

$$
\mathrm{H}_{n}+\mathrm{HI}_{1}+\mathrm{I}_{2} \text { for } \mathrm{H}_{n}+\mathrm{IH}_{1}
$$

Write also

$$
\Delta_{11}+\Delta_{1}+\Delta_{.} \text {for } \Delta_{11}+\Delta_{1} \text {. }
$$

$$
\text { II for } \Pi_{0}+I_{1}+\Pi_{2}, \Delta \text { for } \Delta_{11}+\Delta_{1}+\Delta_{2} \text {, }
$$

and similarly for the other quantities.

Hence

$$
\begin{aligned}
& p^{\prime}=\mathrm{P}-\frac{1}{2} \sigma v^{\prime \prime} \frac{\mathrm{III}}{1 / \mathrm{l}} \\
& \left.\begin{array}{l}
q^{\prime}=Q+\frac{1}{2} \sigma::^{\prime \prime} d \bar{s} \\
w^{\prime}=-\sigma: \Delta
\end{array}\right\}^{\text {the error in each case }} \text { being of order } h^{2}
\end{aligned}
$$

and

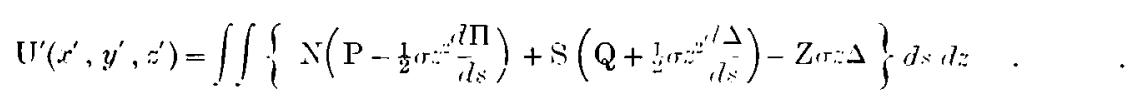

Also from (1) $\Delta-\frac{d Q}{d s}-\frac{\mathrm{P}}{\rho},-\Pi+\frac{d}{d s}-\frac{?}{\rho}$ are of orden $h$, and the formula for $C^{\prime}$ will therefore be correct to the same orler as before if we substitute $\frac{d Q}{d s}+\frac{P}{\rho}$ for $\Delta$, and $\frac{d Y}{d s}-\frac{Q}{\rho}$ for II. We shall also write

Thus

$$
\begin{aligned}
& \int_{-n}^{h} \mathrm{~N} d z=\mathrm{N}_{0}, \quad \int_{-n}^{h} z^{2} \mathrm{~N} / z=\mathrm{N}_{2}
\end{aligned}
$$

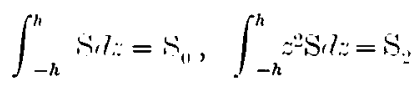

$$
\begin{aligned}
& \int_{-h}^{h} \because Z d z=Z_{1} \text {. }
\end{aligned}
$$

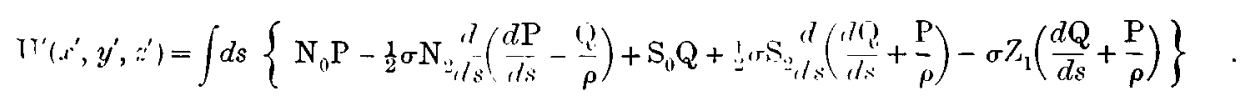

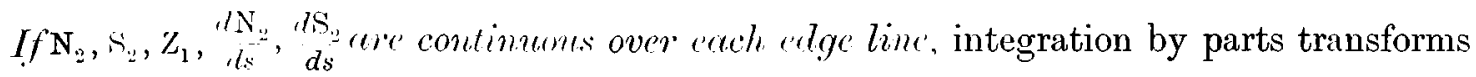
this into

$$
\int d s\left\{\mathrm{P}\left(\mathrm{N}_{0}-\frac{\sigma}{\rho} Z_{1}-\frac{1}{2} \sigma \frac{d^{2} \mathrm{~N}_{2}}{d s^{2}}-\frac{1}{2} \frac{\sigma}{\rho} \frac{d \mathrm{~S}_{2}}{d s}\right)+\mathrm{Q}\left(\mathrm{S}_{0}+\sigma \frac{d Z_{1}}{d s}-\frac{1}{2} \frac{d}{\rho} \frac{d \mathrm{~N}_{2}}{d s}+\frac{1}{2} \sigma \frac{d^{2} \mathrm{~S}_{2}}{d s^{2}}\right)\right\} .
$$


Hence, in order that the permanent mole should be absent from the strain due to $\mathrm{N}, \mathrm{S}, \mathrm{Z}$ the following two conditions are sufficient:-

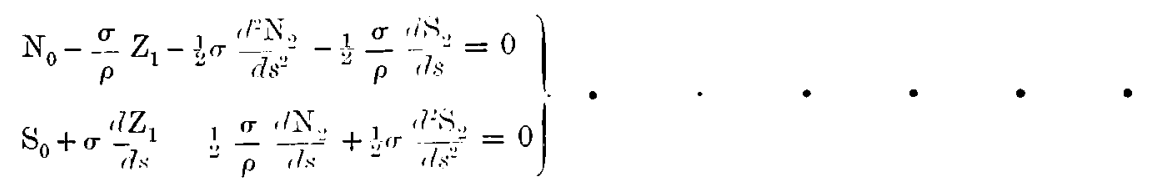

at every point of the edge line or lines.

Further, all systems of traction for which the left-hand members of (7) have given values at every point of an edge will produce the same permanent mode. Now as one such system of traction ue may take the traction dine to w producing, the permanent. mode by itself, as given in $\S 4$ (i). This gives at once the boundlary conditions satisfied by the functions $\mathrm{U}, \mathrm{V}$ of that section, and these boundary conditions, with the internal equations

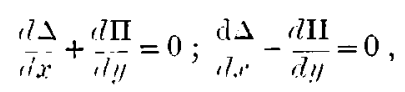

completely define $\mathrm{U}, \mathrm{V}$ which are thus determined, to a third approximation in general. The defining differential equations and surface conditions being practically of the same form as in the familiar first approximation, we need not detail the proof that $U, V$ are actually determinate from the conditions, but pass at once to the important conclusion, an immediate consequence of this determinateness, that the permanent strain will not be absent unless (7) are satisfied, or, in other words, that these conditions are necessary, as well as sufficient. From this again it follows that these conditions are fulfilled, to the order stated, by each of the transitory modes; and this remark is valuable, because, once it has been verified by direct integration, it obviously leads, by an extension of the process of $\$ 42$ (iv), to a completely independent method of dealing with the whole problem. The method is noticeable for its simplicity and directness, but a somewhat serious defect is the rifficuity of arlapting it to the case when the edge stress is discontinuous.

This leads us to consicler the corrcetion that must be ipplied to the integral (6) when the conditions of continuity stated in connection with it are not fulfilled. It will be sufficient to take a case in which breach of continuity occurs at only one point $\mathrm{E}$ of the edge line.

We have defined the positive direction of an edge line in $(k)$; let the excess of the value of $f(s)$ just on the negative side of $\mathbf{E}$ over its value just on the positive side be denoted by $[f()$.$] . Then if \phi(*)$ be continuous

$$
\int f(s) \frac{d}{d s} \phi(s) d s=\phi(s)[f(s)]-\int \phi(s) \frac{d}{d s} f(s) d s,
$$

the integrals, we may suppose, being taken round the elge line from the positive to the negative side of $\mathrm{E}$, and the value of $\phi(s)$ in the integrated term being taken as at $\mathrm{E}$. 
Then to (6) we have in general to ald

or, otherwise arranged,

$$
\begin{aligned}
& \left.-\frac{1}{2} \sigma\left(\frac{d l}{d s}-\frac{Q}{\rho}\right)\left[\mathrm{N}_{2}\right]+\frac{1}{2} \sigma\left(\frac{d(q}{d s}+\frac{\mathrm{P}}{\rho}\right)\left[S_{3}\right]-\sigma Q\left[Z_{1}\right]\right) \\
& +20 \mathrm{p}\left[\begin{array}{c}
d \mathrm{~N} . \\
d s
\end{array}\right]-\frac{1}{2} \sigma\left(s\left[\begin{array}{l}
d s \\
-d s
\end{array}\right]\right.
\end{aligned}
$$

$$
\begin{aligned}
& \left.\mathrm{P}\left\{\frac{1}{2} \frac{\sigma}{\rho}\left[\mathrm{S}_{2}\right]+\frac{1}{2} \sigma\left[\frac{d \mathrm{~N}_{2}}{d \dot{d}}\right]\right\}+q\left\{-\sigma\left[Z_{1}\right]+\frac{1}{2} \frac{\sigma}{\rho}\left[\mathrm{N}_{2}\right]-\frac{1}{2} \sigma\left[\frac{d \mathrm{~s}_{2}}{d x}\right]\right\}\right\} \\
& -\frac{d P^{\prime}}{d s} \frac{1}{2} \sigma\left[\mathrm{N}_{2}\right]+\frac{d g}{d s} 2 \sigma\left[\mathrm{s}_{2}\right]
\end{aligned}
$$

The vilrious terms of this expression may be interpreted with the help of the conceptions of soures and doulhlets. Thus, to go back to (3), we see that the part of $\mathrm{U}^{\prime}$ arising from an element $\mathrm{N}_{11}$ ls of normal traction at $\mathrm{E}$ has $\left(\mathrm{P}_{0}+\mathrm{P}_{1}\right) \mathrm{N}_{1}$ ds for principal term. $P_{0}+P_{1}$ is therefore (principal term of the) value of $U^{\prime}$ due to: 1 unit element of normal force at $\mathrm{E}$. (Since this unit element can only exist in any actual deformation as part of an equilibrating system, the phrase dur to in the last sentence must be taken under reservation. The solution of which $\mathrm{P}_{0}+\mathrm{P}_{1}$ is the $x$-displacement it $\left(x^{\prime}, y^{\prime}, 0\right)$ is in fact maintained by a unit clement of normal traction at $\mathrm{E}^{*}$, acting along with a continuous srstem of form in erfuilibrium with this element, and distributer orer the edge in a manner depending only on the statical value of the element, and not at all on the position of $\mathrm{E}$. For any equilibrating combination of element: the aggregate of these continuous systems will disappear.)

Now the first of the above integrated terms is equivalent to $\left(\mathrm{P}_{0}+\mathrm{P}_{1}\right) \frac{\sigma}{\rho} \cdot[\mathrm{s}:]$.

Hence the discontinuity in $\mathrm{S}_{2}$ at $\mathrm{E}$ has the same effect at a distince from the edge as would have an element of normal traction distributed orer the perpendicular at $\mathrm{E}$ so as to give a resultant $\frac{1}{2} \frac{\sigma}{\rho}\left[\mathrm{S}_{2}\right]$.

Again an element - $\mathbf{A}$ of normal traction at $\mathbf{E}$, combined with an element $\mathrm{A}$ at $\mathrm{E}^{\prime}$, where $\mathrm{EE}^{\prime}=d s$, will give

$$
\begin{gathered}
\mathrm{U}^{\prime}=\mathrm{A} \frac{d}{d s}\left(\mathrm{P}_{0}+\mathrm{P}_{1}\right) d s \\
=\frac{d}{d s}\left(\mathrm{P}_{0}+\mathrm{P}_{1}\right), \text { if we take } \Lambda l_{s}=1 .
\end{gathered}
$$

$\frac{l}{d s}\left(P_{1}+P_{1}\right)$ is therefore due to a unit doublet of normal force it $\mathrm{H}$, and from the term $-{ }_{d:} \mathrm{P}_{2}, r\left[\mathrm{~N}_{2}\right]$ we conclurle that the discontinuity in $\mathrm{N}_{2}$ at $\mathrm{E}$ has the same interior effect as a doublet of normal force at $\mathrm{E}$ of strength $-!_{2} \sigma\left[\mathrm{N}_{2}\right]$.

The other terms may be interpreter similinly. It rloes not seem posille to account on physicil grounds for any exerpt the prineipal terms of the solution given above. The principal terms are of course the simm as those derlucerl in the ondinary theory from the 'Principle of the elastic equivilence of statically "ipuipollent srstems of loul.'

TRANS. ROY. SOG: EDIN, VOL. XLI. PART I. (NO. 8) 
With reference to the equivalence of mere discontinuities to line elements and doublets one or two remarks may be made. Discontinuity in the applied force will not produce infinite displacement at a line where it takes place, but a line element of load, and, a fortiori, a doublet, will do so. The permanent mode may therefore contain infinities at the edge which do not exist in the exact solution. There is really no difficulty in this, since the permanent mode does not purport to represent the strain, even approximately, in the immediate vicinity of the edge. The point may be illustrated by the permanent part of the infinite solid solution for a single force. This becomes infinite on the perpendicular through the source in a totally different way from the exact solution. A good deal of discussion took place at one time over a similar point in the flexural solution. This will be referred to again, but the considerations we have adduced seem to remove the chief part of the difficulty.

\section{Flexural strain.}

In this case $\mathrm{N}, \mathrm{S}$ are odd functions, and $\mathrm{Z}$ an even function of $z$.

(i) Permanent mode.

This mode is defined in terms of one function $\mathrm{F}$ of $(x, y)$ satisfying $\nabla^{4} \mathrm{~F}=0$, and may be referred to simply as an $\mathrm{F}$ strain.

$$
\begin{aligned}
& \phi=-\left(\because \mathrm{F}-\frac{1}{6} z^{3} \nabla^{2} \mathrm{~F}\right) ; \theta=z \mathrm{~F}-\frac{1}{6} z^{3} \nabla^{2} \mathrm{~F}-2 \lambda^{2} z \Gamma^{2} \mathrm{~F} \\
& \left.\begin{array}{l}
p=\frac{d}{d n} \\
q=\frac{d}{d s}
\end{array}\left\{-4(1-\sigma)\left(z \mathrm{~F}-\frac{1}{d} z^{3} \nabla^{2} \mathrm{~F}\right)+2\left(\frac{1}{3} z^{3}-1\right)^{2}\right) \nabla^{* 2} \mathrm{~F}\right\} \\
& w=4(1-\sigma)\left(\mathrm{F}-\frac{1}{2} z^{2} \nabla^{2} \mathrm{~F}\right)+2\left(\ddot{\prime}^{\prime}-l^{2}\right) \Gamma^{2} \mid \mathrm{K}
\end{aligned}
$$

For shortness in writing out the stresses, we shall work with symbols $\vartheta_{1}, \vartheta_{3}, \vartheta_{3}$, denoting operations of differentiation applied to $\mathrm{F}$, and defined by the equations

Then

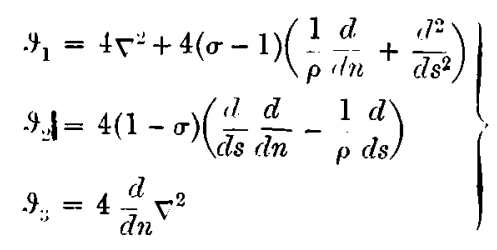

$$
\begin{aligned}
& \frac{\min }{2 \mu}=-y_{1} \mathrm{~F}+\left\{\frac{1}{6} z^{3}+\frac{1}{2} \frac{1}{1-\sigma}\left(\frac{1}{3} \cdot l^{3}-h^{2}\right)\right\} \cdot \overline{\nabla^{2} \mathrm{~F}} \\
& { }_{\nu_{\mu}}^{n s}=-\ldots \mathrm{y} F+\left\{\frac{1}{6} z^{3}+\frac{1}{2} \frac{1}{1-\sigma}\left(\frac{1}{3} z^{3}-h^{2} z\right)\right\} \cdot \vartheta_{2} \nabla^{2} F \\
& \frac{\hat{n z}}{\mu 2}=\frac{1}{2}\left(\because^{2}-h^{2}\right) 9_{3} \mathrm{~F}
\end{aligned}
$$


(ii) Rotational trensitory mode's.

These are as in $\$ 42$ (ii), except that now

$$
\begin{aligned}
& \psi=\sum_{n=0} \psi_{n}(x, y) \sin ^{2} n+1 \pi \\
& \Gamma " \psi_{n}-(\ddot{\prime} n+1)^{2}{ }_{4 l^{2}}^{\pi^{2}} \psi_{n}=0 .
\end{aligned}
$$

(iii) Dilatutional transitory morl's.

As in $\$+2: 2$ (iii),

$$
\phi=\sinh \kappa: .: f^{\prime}(x, y) ; \theta=-\cosh 2 \kappa h \cdot \phi
$$

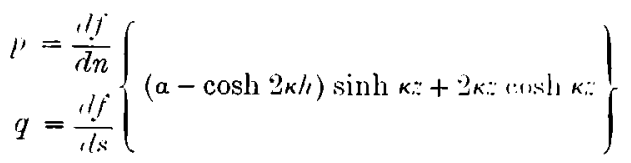

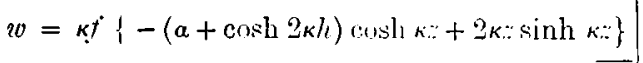

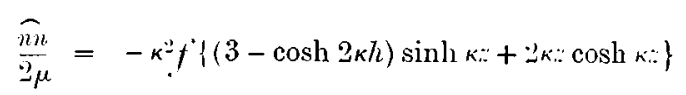

$$
\begin{aligned}
& -\left(\frac{1}{\rho} \frac{d f}{d i n}+\frac{d y t}{d s^{2}}\right)\{(a-\cosh 2 \kappa h) \sinh \kappa+2 \kappa * \cosh \kappa z\}
\end{aligned}
$$

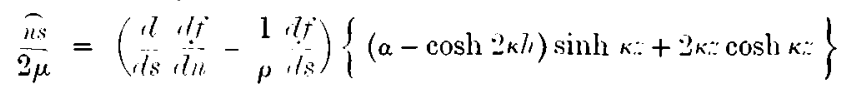

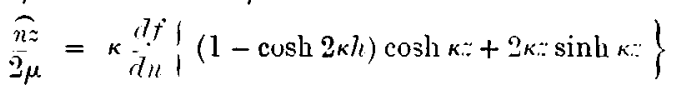

We have $\frac{d f}{d n}=-i \kappa f$ to the lowest order,

$$
\text { or } f=\begin{array}{ll}
i & d f \\
\kappa & d n
\end{array}
$$

Hence if we put

$$
\left.\begin{array}{l}
\kappa \frac{d f}{d n}=y_{\kappa} \\
i\{(\cosh 2 \kappa h-3) \sinh \kappa z-2 \kappa z \cosh \kappa:\}=\mathrm{N}(\kappa z) \\
\quad(1-\cosh 2 \kappa h) \cosh \kappa z+2 \kappa ; \sinh \kappa z=Z(\kappa z)
\end{array}\right\}
$$

the above strain gives

$$
\begin{aligned}
& \left.\begin{array}{l}
\frac{\hat{n n}}{2 \mu}=g_{\kappa} \mathrm{N}(\kappa:) \\
\widehat{n s}=0
\end{array}\right\} \text { with an error of relative order } h, \\
& \frac{\widehat{n} \tilde{z}}{2 \mu}=g_{\kappa} Z(\kappa) \text { exactly, }
\end{aligned}
$$

and we may note that $\int_{-n}^{n} \pi \mathrm{N}(\kappa ;)=0$ and $\int_{-h}^{h} Z(\kappa), r:=0 . \quad(\$ 41$.

The same remarks as in the extensional case might be made here about the complementary character of the types (ii), (iii) in regard to their contributions to edge displacement or traction, when $h$ is small.

(iv) If we follow the lines of the discussion of the extensional case, we have now to consider the approximate allocation of a given system of edge traction among the three types of strain. 
The investigation is this time more complex, chiefly in consequence of the presence of the stress $\widehat{n}:$ in the permanent mode. Since, moreover, flexure is much more important physically than extension, we shall give a fairly detailed discussion in the next article, but in the meantime we may examine what could be done with a solution in which, as in $\S+2$ (iv), the principal part of the edge traction is of order zero for each type of strain, and the parts of higher order are neglected.

Such a solution would give

$$
\begin{aligned}
& \mathrm{N}=\mathrm{N}_{\mu} \quad+\mathrm{N}_{a} \\
& \mathrm{~s}=\mathrm{S}_{\mu}+\mathrm{S}_{r} \quad Z_{d}
\end{aligned}
$$

But we see at once that we do not in this way get a perfectly general distribution of $N, S, Z$, since the list equation gives $\int_{-h}^{h} Z i z=0$. A closer examination is therefore necessary, and it will perhaps conduce to lucidity if we consirler separately the three ases of normal, tangential, and perpendicular traction.

(i) Normal traction.

\section{Flexural forces.}

$\mathrm{N}$ being of order zero, we can satisfy the conditions by taking $z \mathrm{~F}, \frac{d^{2} \psi}{d z^{2}}$, and $g_{x}$ all of this order, but besides the terms of order zero in the stresses, it will be necessary to take account of the terms of $\widehat{n z}$ which come from $\mathrm{F}$ and $\psi$, albeit these are of an order one higher. Then

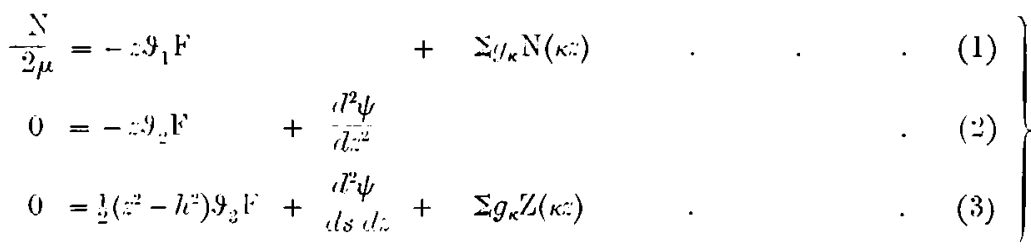

Assuming these provisionally, multiply (1) by $\approx$ and integrate from $-h$ to $h$.

$$
\begin{aligned}
& \text { 'Thus: } \\
& \vartheta_{1} \mathrm{~F}=-\frac{3}{4 \mu h} \int_{-h}^{h}: \mathrm{N} / z
\end{aligned}
$$

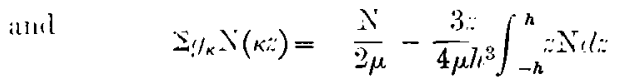

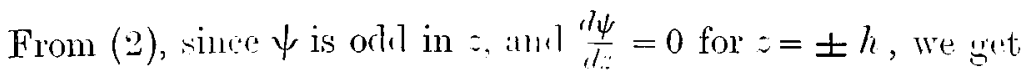

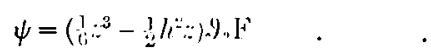

In (3) the rerms are of different orrers; thus, with the help of $(6)$,

$$
\begin{aligned}
& y_{j} \mathrm{~F}+\frac{d}{d s} y_{1} \mathrm{~F}=0 \\
& \because g_{k} Z(\kappa: i)=0 \quad \text {. . . }
\end{aligned}
$$

(4) anl (7) define F, (6) then gives the exlge vilue of $\psi$, ind (5), (8) determine $y_{k}$,

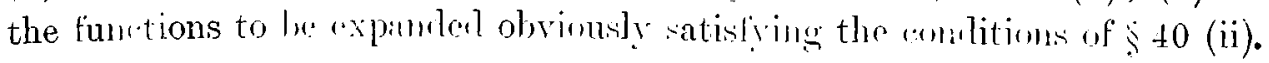


Further, with the values of $F, \psi, g_{x}$ sin determined, thr apuations (1), (2), (3) are

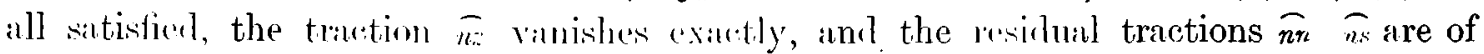
order 1.

(ii) Tangential trection.

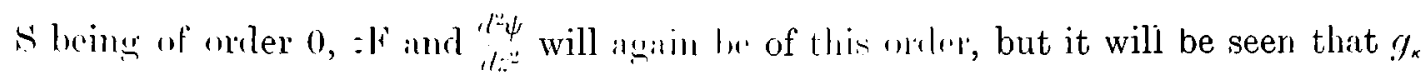
is of order 1. For, making these supprisitions, we have

$$
\begin{aligned}
& 0=-\because y_{1} \mathrm{~F} \quad \text {. . . . (1) }
\end{aligned}
$$

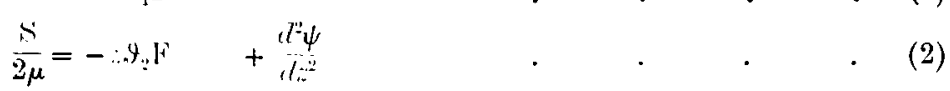

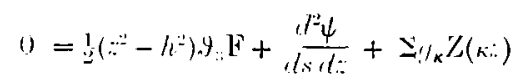

$$
\begin{aligned}
& \text { From (1), } \quad 9_{1} \mathrm{~F}=0
\end{aligned}
$$

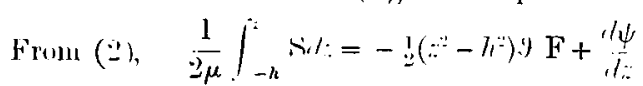

Difterentiate this with resperet to $s$, amd sulitriat from (3). Thus

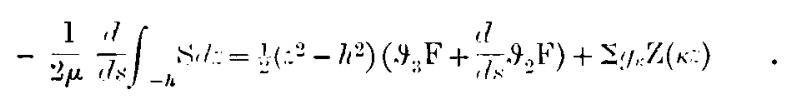

and, integrating the list from $-l$, to $h$,

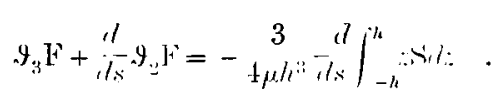

$(4)$ and $(5)$ define F, and (5) integriter from 0 to $z$ wives $\psi$. With the values so determined, and with $(6)$ satisfied hy $y_{\kappa}$ (and this is posilile in virtue of $(7)$ ), the equations (1), (2), (3) are all sitistierl, the trintion $\tilde{n}$ : ranishes ractly, and the residual tractions $\widehat{n n}, \widehat{n s}$ are of orlere 1 .

By combining this with the preceling case, we see that the results do in fact give it first approximation to the solution, since the residual stresses $N, S$ are ear.h of all order higher than their original wiven values.

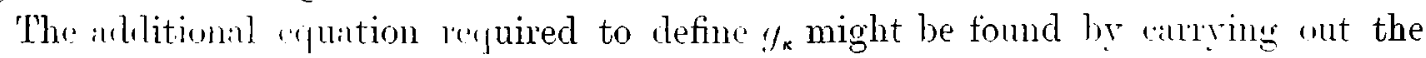
procese of (i) with the resilual normal traction $4 \mu \frac{d}{d s} \frac{d \psi}{d n}$. The allalysis would be practically the sime as will he oriven in connection with the next alse.

(iii) Prependicular traction.

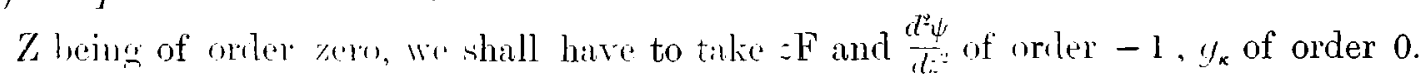

On this hypothesis, we shall write lown the watet explession for $\tilde{n}$, and the terms of

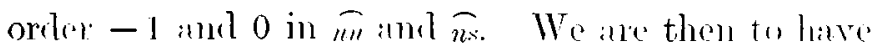

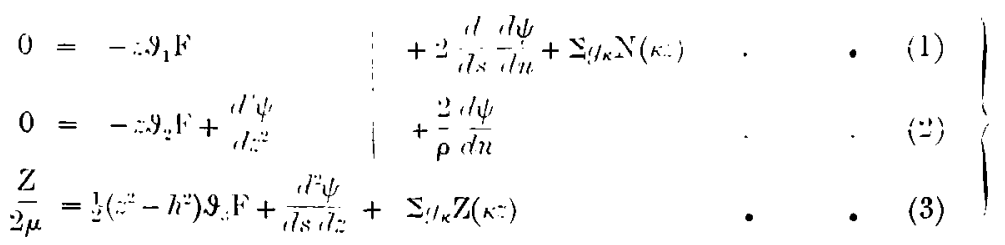


In the first two stresses the terms of order -1 are annulled by taking

$$
\begin{aligned}
& 9_{1} \mathrm{~F}=0 \quad \text {. } \quad . \quad \text {. }
\end{aligned}
$$

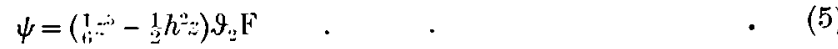

Then integration of (3) from $-h$ to $h$ gives

$$
\left(9_{3}+\frac{d}{d s} \cdot 9_{3}\right) \mathrm{F}=-\frac{3}{4 \mu h^{3}} \int_{-n}^{h} \mathrm{Znl}: \quad \text {. . . . }
$$

and from this with (3),

$$
\Sigma g_{\kappa} Z(\kappa)=\frac{Z}{2 \mu}+\frac{3}{8 \mu h^{3}}\left(\because \cdots-h^{2}\right) \int_{-h}^{h} Z l i
$$

Another equation is required before $g_{\kappa}$ is defined, but in view of the results of (i), (ii) we can already infer that the values of $\mathrm{F}, \psi$ determined by $(4),(6)$ and (5) are the correct principal values, since the residual stresses $\widehat{n n}, \widehat{n s}$ are of order zero. As for $g_{x}$, we note that we cannot complete its definition by annulling the residual stress $\widehat{n n}$, for the condition $\int_{-h}^{h} 2 \frac{d}{d s} \frac{d \psi}{d n} s d z=0$ is not satisfierl. In order to get the remaining equation for $g_{k}$ we must therefore solve by the method of (i) for the residual normal traction $4 \mu \frac{d}{d s} \frac{d \psi}{d n}$, so as to get the equation corresponding to (i) (5). The matter might be left at this stage, but it may be interesting actually to carry out the process of balancing the residual parts of $\widehat{n n}$ and $\widehat{n} s$. By so doing we shall not only define $g_{k}$, but also obtain a second approximation to $\mathrm{F}$ and $\psi$.

We have to introxluce into the solution $\mathrm{F}^{\prime}, \psi^{\prime}, g_{x}^{\prime}$ with $z \mathrm{~F}^{\prime}$ and $\frac{d^{2} \psi^{\prime}}{d z^{\prime \prime}}$ of order $0, g_{x}^{\prime}$ of order 1 . The equations to lx satisfied are

$$
\begin{aligned}
& -2 \frac{d}{d s} \frac{d \psi}{d n}-\Sigma g_{k} \mathrm{~N}(\kappa)=-2 y_{1} \psi^{\prime} \quad \text {. . . . (8) }
\end{aligned}
$$

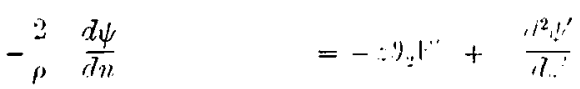

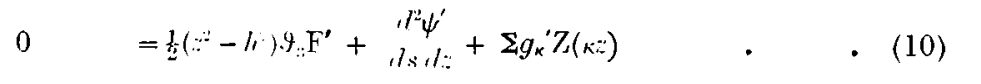

We must first find the principal value of $\frac{d \psi}{d n}$ in terms of defined quantities.

Now from (5),

$$
\psi=\left(\frac{1}{6} z^{3}-\frac{1}{2} / l^{2} ;\right) \cdot 9 . \mathrm{F}, \text { at the edge. }
$$

Hence, by Fourier's Theorem,

$$
, \psi=-\frac{32}{\pi^{4}} h^{3} \vartheta_{2} \mathrm{~F}\left(\sin \frac{\pi \cdot:}{2 h}-\frac{1}{3^{4}} \sin \frac{3 \pi i:}{2 h}+\frac{1}{5^{4}} \sin \frac{5 \pi::}{2 h}-\ldots\right)
$$

Within the plate, therefore,

$$
\psi=-\frac{32}{\pi^{\prime}} h^{2}\left(\psi_{0} \sin \frac{\pi z}{2 h}-\frac{1}{3^{3}} \psi_{1} \sin \frac{3 \pi z}{2 h}+\frac{1}{5^{4}} \psi_{2} \sin \frac{5 \pi \%}{2 h}-\ldots\right)
$$


where

$$
\Gamma^{\prime \prime} \psi^{\prime \prime m}-\frac{(2 ! n+1)^{2} \pi^{2}}{4 h^{2}} \psi_{m}=0
$$

and $\psi_{m}$ has the edge value $\vartheta_{2} \mathrm{~F}$.

'Thus

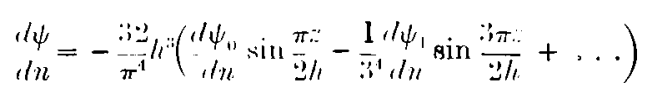

the principal value of which is

Let

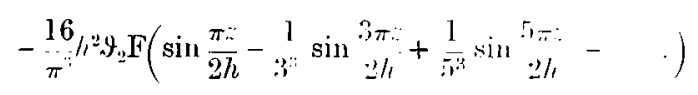

then

$$
\beta(z / h) \equiv \frac{16}{\pi^{3}}\left(\sin \frac{\pi *}{2 h}-\frac{1}{3 i} \sin \frac{3 \pi:}{2 h}+\frac{1}{5^{3}} \sin \frac{5 \pi *}{2 / h}-\ldots\right)
$$

ant

$$
\frac{d \psi}{d n}=-h: 3(z / h) \cdot y_{2} \mathrm{~F}
$$

$$
\begin{aligned}
& \int_{-h}^{h} z \frac{d \psi}{d n} d z=-\frac{1}{\pi^{5}} h^{4}\left(1+\frac{1}{3}+\frac{1}{5 j}+\ldots\right) \cdot 9 F \\
& =-\frac{128}{\pi^{3}} \gamma_{3} \cdot l^{4} \cdot y_{2} \mathrm{~F} \\
& \text { if } \\
& \gamma_{5} \equiv 1+\frac{1}{3^{5}}+\frac{1}{5^{5}}+\ldots
\end{aligned}
$$

Now multiply (8) by $z$, and integrate from $-h$ to $h$.

\section{Hence}

and

$$
\vartheta_{1} \mathrm{~F}^{\prime}=-\frac{384}{\pi^{5}} \gamma_{5} l \frac{d l}{\lambda_{1}} \cdot 9_{2} \mathrm{~F} \quad . \quad . \quad .
$$

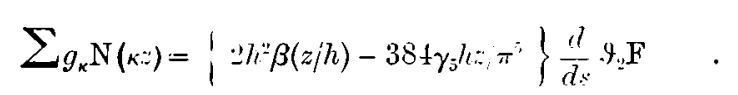

This, and equation (7), define $g_{k}$.

As we do not require $g_{k}^{\prime}$, we will climinate it from (10) at once by integrating. Thus

$$
0=-2 \frac{l^{\prime \prime}}{3} 9_{3} \mathrm{~F}^{\prime}+\frac{d}{d !}\left[\psi^{\prime}\right]_{-h}^{h}
$$

Multiply (9) by $z$ and integrate. Then

and from this with the last

$$
-\frac{2}{\rho} \int_{-h}^{n}: \frac{d \psi}{d l u} d:=-\frac{2 l^{3}}{3} 9_{3} \mathrm{~F}^{\prime}-\left[\psi^{\prime}\right]_{-h}^{h}
$$

or, from (13),

$$
\frac{d}{d s}\left\{\frac{2}{\rho} \int_{-h}^{h} z \frac{d \psi}{d u} d z\right\}=2 \frac{l^{3}}{3}\left(y_{2} \mathrm{~F}^{\prime}+d_{d s}^{\prime} y_{-} \mathrm{F}^{\prime}\right)
$$

$$
y_{3} \mathrm{~F}^{\prime}+\frac{d}{d s}, y_{y} \mathrm{~F}^{\prime \prime}=-\frac{384}{\pi^{b}} \gamma_{5} h \frac{d}{d s}\left(\frac{1}{\rho}, y_{3} \mathrm{~F}^{\mathrm{i}}\right)
$$

(14) and (16) give $\mathrm{F}^{\prime}$, and $\psi^{\prime}$ may be found from (9). 
If we write $\overrightarrow{\mathrm{F}}$ for $\mathrm{F}+\mathrm{F}^{\prime}$, we get from (4), (14)

$$
\begin{aligned}
\vartheta_{1} \overline{\mathrm{F}} & =-\frac{384}{\pi^{5}} \gamma_{5} l \frac{d}{d s} \vartheta_{2} \bar{F} \\
& =-\frac{384}{\pi^{5}} \gamma_{6} l d \frac{d}{d l s} \vartheta_{3} \overline{\mathrm{F}}
\end{aligned}
$$

neglecting the term in $h \mathrm{~F}^{\prime}$, which is of order $h^{2}$ relative to $\overrightarrow{\mathrm{F}}$.

This may be written

$$
\left(\vartheta_{1}+\frac{384}{\pi^{5}} \gamma_{5} h \frac{l}{d s} 9_{2}\right) \overline{\mathrm{F}}=0
$$

Similarly from (6), (16) we obtain

$$
\left(\vartheta_{3}+\frac{l}{d s} y_{2}+\frac{384}{\pi^{5}} \gamma_{5} h \frac{d}{d s} \frac{1}{\rho} y_{2}\right) \overline{\mathrm{F}}=-\frac{3}{4 h^{3}} \int_{-n}^{h} z_{t} l l:
$$

We may regard (17) and (18) as the equations giving $F$ to a second approximation. If we combine the results of the three case : of this article, we obtain

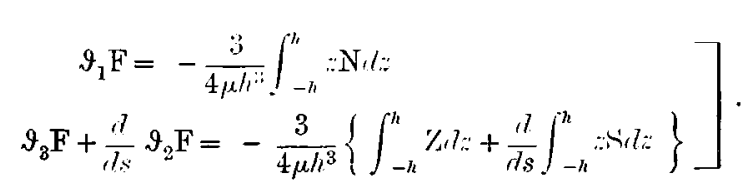

These are the equations usually referred to as Kirchhoff's boundiry conditions. The extension of the more approximate conditions (17), (18) to the general cise will be given in the next article.

46. Flexural strain under given edge trations. The triben's firmetion wethod fin the permucerint mods.

The displacement at $\left(x^{\prime}, y^{\prime},:^{\prime}\right)$ due to tractions $N, s, Z$ is defined in $\S 33$, II. (i), in terns of the work difference from the system

$$
\begin{aligned}
& \psi=-\left(332 \pi \mu h^{3}\right)\left(\because \gamma-1 \nabla^{2} \chi\right) \\
& t=\left(3 / 32 \pi \mu h^{3}\right)\left(\because \chi-1 ; \Gamma^{2} \chi-2 / \cdots \Gamma^{2} \chi\right) .
\end{aligned}
$$

From this system let the terms conveying resultint strens $h_{0}$ removed the resilue is still an $\mathrm{F}$ strain with $\mathrm{F}=\mathrm{F}_{0}$ say, and $\mathrm{F}_{0}$ is of order $h^{-3}$.

Wo have to balance $\mathrm{F}_{0}$ at the edge, and the edige displicements in thr halanced solution being $\mu^{\prime}, q^{\prime}$, $\prime^{\prime \prime}$, the work difference required $\left(\mathrm{F}_{1}\right.$ of $\left.\$ ?: 3\right)$ is

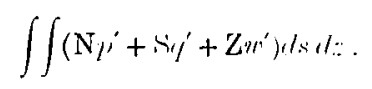

'The problem is to detenine $f^{\prime}, q^{\prime}, m^{\prime}$ as clowily as is puaticable.

The tractions to lo linliumen are

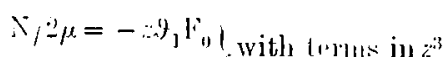

$$
\begin{aligned}
& \left.\dot{s} / 2 \mu=-\therefore \psi_{2} \mathrm{H}_{0}\right\}
\end{aligned}
$$

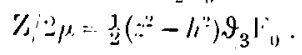




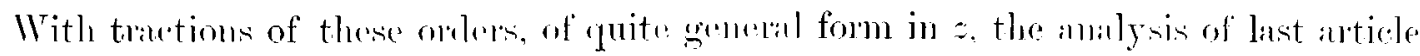

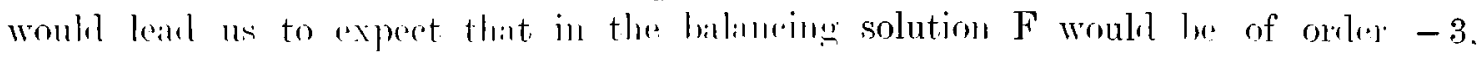

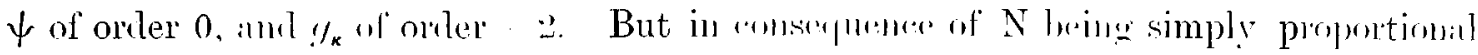

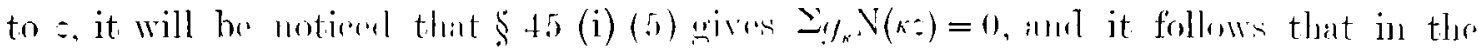

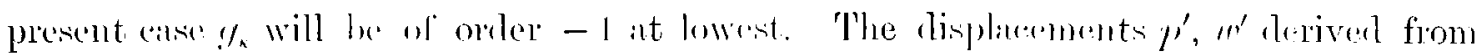

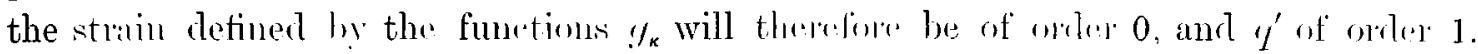

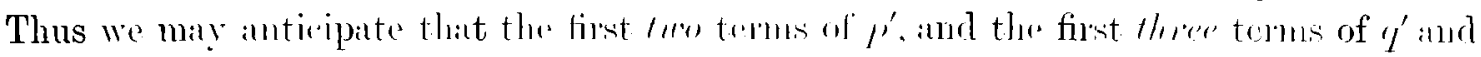

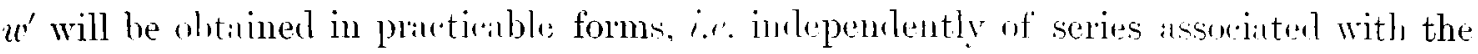
zeroes of $\sinh \ddot{2}_{k} h-2 \times h$.

'The tratetions written down alove may be balancen approximately by strains $F^{\prime}, \psi^{\prime}$. We require

These are equivilent to

$$
\begin{aligned}
& \begin{array}{l}
-\therefore y_{1}\left(\mathrm{~F}_{11}+\mathrm{F}^{\prime \prime}\right)=0 \\
-\therefore y_{2}\left(\mathrm{~F}_{0}+\mathrm{F}^{\prime}\right)+r^{\prime \prime} \psi^{\prime}=0
\end{array}
\end{aligned}
$$

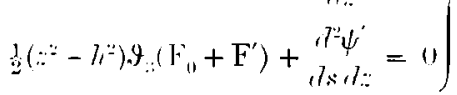

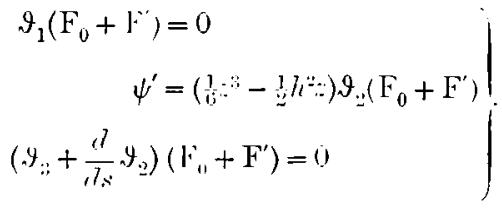

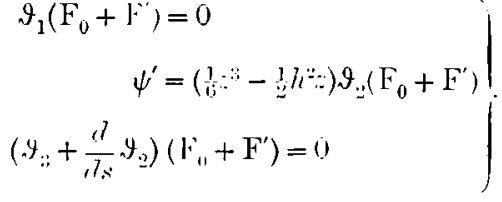

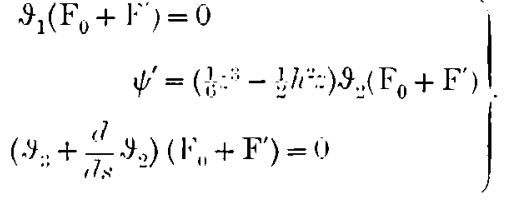

which determine $\mathrm{F}^{\prime}, \psi^{\prime}$.

The principal terms of the resiclual tractions are

$$
\begin{aligned}
& \left.\frac{\widehat{n n}}{2 \mu}=2 \frac{l}{d s} \frac{d \psi^{\prime}}{d n}=-2 / \cdots \beta(\cdots h) \frac{d}{d s} \cdot g_{2}\left(\mathrm{~F}_{0}+\mathrm{F}^{\prime}\right)\right) \\
& \frac{\overrightarrow{n s}}{2 \mu}=\frac{2}{\rho} \frac{d \psi^{\prime}}{d n}=-\frac{\partial 2 \lambda^{2}}{\rho} \beta(\cdot ; h) \cdot g_{2}\left(\mathrm{~F}_{11}+\mathrm{F}^{\prime}\right) \quad j
\end{aligned}
$$

as in (iii) (1:) of last article, and ther may be dealt with in the manner illustrated there.

To balance them take strains definel by $\mathrm{F}^{\prime \prime}, \psi^{\prime \prime}, g_{x}^{\prime \prime}$ with $: \mathrm{F}^{\prime \prime} .{ }^{\prime \prime \prime} \psi^{\prime \prime} \cdot \eta^{\prime \prime} \cdot g_{x}^{\prime \prime}$ of order -1 .

We must therrowe have

From these, as in last alticlu.

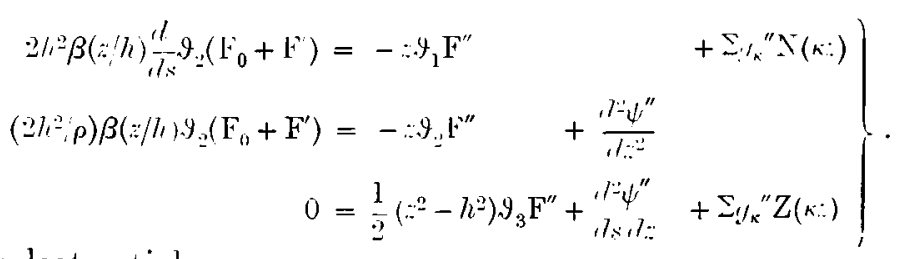

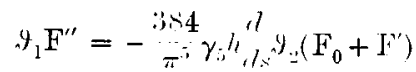

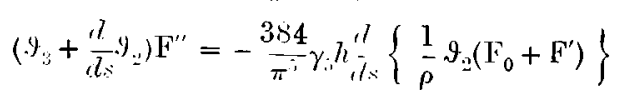

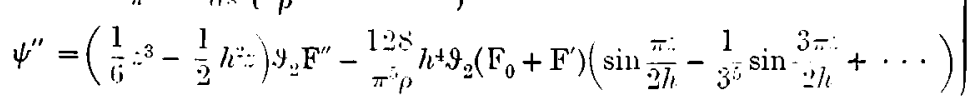

TRANS. ROY. SOC. EDIN., VOL. XLI. PART I. (NO. 8). 
whence

$$
\frac{d \psi^{\prime \prime}}{d n}=-h^{2} \beta(z / h) 9_{2} \mathrm{~F}^{\prime \prime}+\frac{2}{\rho}\left(\frac{1}{6} z^{3}-\frac{1}{2} h^{2} z\right) \vartheta_{2}\left(\mathrm{~F}_{0}+\mathrm{F}^{\prime}\right)
$$

We are now left with tractions $\widehat{n n}, \widehat{n s}$ of order zero. In the solution balancing these, $\mathrm{F}^{\prime \prime \prime}$ will be of order $-1, \psi^{\prime \prime \prime}$ of order $2, g_{k}^{\prime \prime \prime}$ of order 0 . We do not think it worth while to write down the equations defining $\mathrm{F}^{\prime \prime \prime}$, but it should be noticed that they can be found explicitly. In fact, although the residual tractions $\widehat{n n}, \widehat{n s}$ with which we are now dealing are partly defined by $\kappa$ series, the integrals $\int_{-h}^{h} z \widehat{n n} d z$ and $\int_{-h}^{h} z \widehat{n s} d z$ will be found to vanish to the order concerned so far as they come from these series, in virtue of the relation $\sum g_{\kappa}^{\prime \prime} Z(\kappa z)=0$, which follows from (iii) (7) of last article.

The functions $g_{x}^{\prime \prime}$ give terms of order zero in $y^{\prime}, u^{\prime}$.

$$
\begin{array}{rccc}
\text { Hence, including in } & \eta^{\prime} & \text { terms of orders } & -2,-1 \\
\ldots \ldots & q^{\prime} & \ldots \ldots & -2,-1,0 \\
\ldots \ldots & u^{\prime} & \ldots \ldots & 3,-2,-1
\end{array}
$$

we have

$$
\left.\begin{array}{l}
p^{\prime}=-4(1-\sigma): \frac{d}{d u}\left(\mathrm{~F}_{0}+\mathrm{F}^{\prime}+\mathrm{F}^{\prime \prime}\right) \\
q^{\prime}=-4(1-\sigma): \frac{l}{d s}\left(\mathrm{~F}_{0}+\mathrm{F}^{\prime}+\mathrm{F}^{\prime \prime}+\mathrm{F}^{\prime \prime \prime}\right)+\left(\frac{4-2 \sigma}{3} z^{3}-2 l l^{\prime 2}\right) \frac{d}{d s^{\prime}} \nabla^{2}\left(\mathrm{~F}_{0}+\mathrm{F}^{\prime}\right) \\
-2 \frac{d}{d n}\left(\psi^{\prime}+\psi^{\prime \prime}\right) \\
u^{\prime}=4(1-\sigma)\left(\mathrm{F}_{0}+\mathrm{F}^{\prime}+\mathrm{F}^{\prime \prime}+\mathrm{F}^{\prime \prime \prime}\right)+2\left(\sigma \varkappa^{\prime \prime}-l \iota^{2}\right) \nabla^{2}\left(\mathrm{~F}_{0}+\mathrm{F}^{\prime}\right) .
\end{array}\right\}
$$

The value of $\frac{d \psi^{\prime}}{d n}$ to a second approximation is

$$
\frac{d \psi^{\prime}}{d n}=-l^{2} \beta(z / h) \cdot 9_{2}\left(\mathrm{~F}_{0}+\mathrm{F}^{\prime}\right)-\frac{1}{2 \rho}\left(\frac{1}{6} z^{3}-\frac{1}{2} l l^{2} \cdot\right) \cdot 9_{2}\left(\mathrm{~F}_{0}+\mathrm{F}^{\prime}\right)
$$

and $\frac{d \psi^{\prime \prime}}{d n}$ is given by $(5)$.

The function $F_{1}$ which $(\$ 33)$ defines the permanent solution is

$$
\iint\left(\mathrm{N} p^{\prime}+\mathrm{S} q^{\prime}+Z w^{\prime}\right) d s d z
$$

For the case of a solid or hollow circular plate all the quantities in the right-hand members of (6) can actually be calculated, and we thus obtain the solution for normal traction to a secomel approximation, and for tangential or perpendicular traction to a third approximation, in a form, moreover, applicable without modification or addition even when the given edge stresses are discontinuous.

We conclude by deducing the equations rorresponiling to Kirchhoff's boundary conditions to a second approximation. (They might he found to one order higher in the case of vanishing normal traction.)

We suppose that the given tractions $\mathrm{N}, \mathrm{S}$ are of the sime order in $h$, and that $Z$ is of an orler onc higher. Any case may be reduced to combinations of cases satisfying this condition. 
If we write $F^{\prime}$ lon $F_{10}+F^{\prime}+F^{\prime \prime}$, then it follows from (6), (7), (8) that the terms of two lowest orkins in $F_{1}$ alde given by

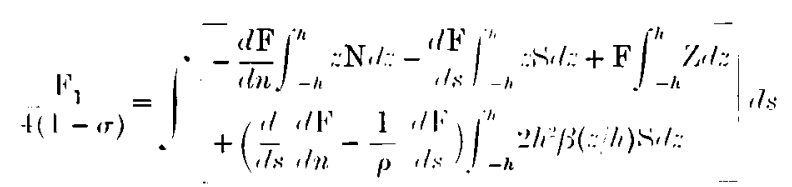

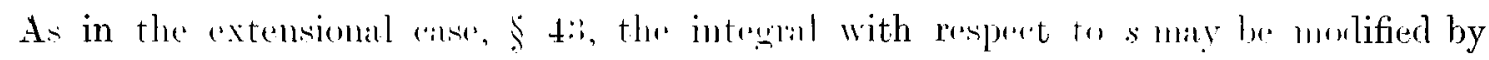

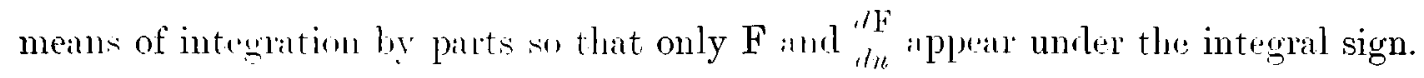

II rite

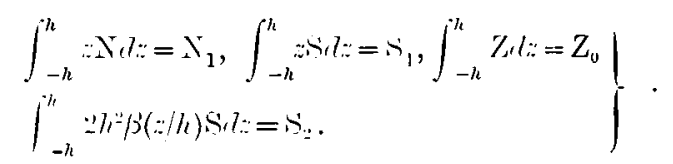

Then if $\mathrm{N}_{1}, \mathrm{~S}_{1}, \mathrm{Z}_{0}, \mathrm{~S}_{2}$ arc continuous functions of $s$,

$$
\frac{\mathrm{F}_{1}}{t(1-\sigma)}=\int\left\{-\frac{d \mathrm{~F}}{d n}\left(\mathrm{x}_{1}+\frac{d s}{d s}\right)+\mathrm{F}\left(\frac{d s}{d x}+Z_{0}+\frac{d s}{d s}\right)\right\} d s
$$

Hence any two systems of trution will give the same permanent mode to a second approximation, provided the values of $x_{1}+\frac{d s}{d s}$ and $\frac{d s_{1}}{d s}+Z_{n}+\frac{d}{d s}$ are the same for the two systems.

Now for the system $F_{1}, \$ 44$ (i),

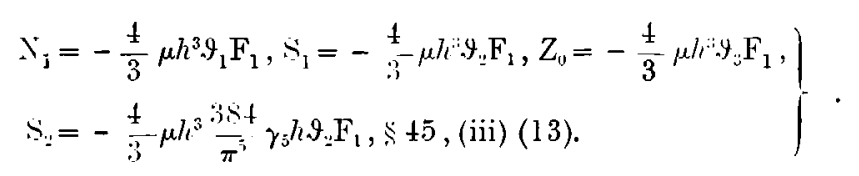

Thus, with $\vartheta_{1}, \vartheta_{2}, \vartheta_{2}$ defined as in $\$ 44$ (i), the boundary conditions are

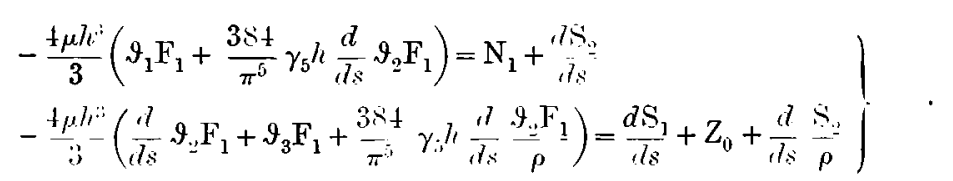

When only the principal terms are retained, these reduce to Firchhoff"s comditions.

If $S_{1} n S_{2}$ is riscontinuous at any point of the edge. integrater terms will appear in equation (11), as in the extensional case, $\$ 43$. Thus, if the normal couple sis be discontinuous at a point $\mathrm{P}\left(x_{=} s^{\prime}\right)$, there will appear wn the riglit of $(11)$ a term

$$
F\left(s^{\prime}\right)\left|s_{1}\right|_{s=s^{\prime}}^{s=}
$$

'The methor of dealing with such a discontinuity in any actual problem is obvious, for

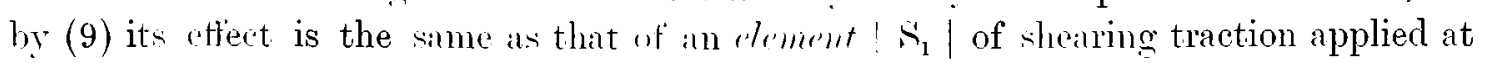
P. a result which on the 'elastic equivalence' thenry may easily be obtained by a trifling modification of the process by which THonson and Tait reconciled the conditions of Kirchhoff and Potsson. 


\section{Addition to Paper ey J. Doutall on- \\ "An Analytical Theory of the Equilibriom of an Isotropic Elastic Plate."}

(Note added May 20, 1904.)

The kindness of one of the referees enables me to supply the following references to recent work bearing on the subject of the paper.

(a) J. H. MicheLL, in a paper "On the direct determination of Stress in an Elastic Solid, with application to the Theory of Plates," Proc. Lond. Matll. Six., vol. 31. 1899, shows how the stresses might be found without prerious determination of the displacements. In the case of the stress $\ldots$ or $R$, he finds that $\nabla^{4} R$ is a given function in the body of the plate, while $\mathrm{R}$ and $d \mathrm{R} / \mathrm{r}$ are given on the faces. If we neglect the conditions at the edge, which have practically no influence on the result, a value of $\mathrm{R}$ satisfying these conditions can be found, in terms of Fourier integrals for instance. Mr MICHell does not determine $\mathrm{R}$-this has been done in the present paper-but proceeds to deduce the forms of the remaining stresses, and the differential equition for the normal displacement of a point on the mid plane. One special case of normal force is worked out to a first approximation, and Lagrange's equation for this case deduced.

For the conditions at the edge, reference is made to the ordinary ThomsonBoussinesq theory, which uses the principle of equipollent loads.

(b) L. N. G. FrLon, "On an ipproximite solution for the bending of a Beam of rectangular cross section under any system of Loml, with special reference to points of concentrated or discontinuous Loading," Phil. Trans. R. Sor. Lomel. (Sec. A), vol. 201 (1903).

Dr Frcon's solution applies to a beam in which the ratios of breadth to depth, and . of depth to length, are both small. The axis of : being taken in the direction of the breadth, the stress $-i$ is taken as negligible, and equations are deduced for the mean values, across the breadth, of the displacements $" x, r$. These erfuations are the same as equations (90), page 182 of the present paper, with the horly force null. In order to see the reason of this from our standpoint, we may notice that the assumption that :-.: vanishes eliminates all the solutions of whit we have called the dilatitional trinsitory type, and that taking the mean of the displacements eliministes all the flexural solutions. as well as the rotational transitory solutions:-

As regards the conditions at the ends, the beam is treater as a long rod.

It may be of interest to remark that tle results of $\$ 43$ above furnish the data for a more approximate treatment of the problem on the lines followed by Dr FiLos.

(c) A note appended to a pirper by Profensor Lane in I'ror. Lomer. Mreth. Soc.. vol. $34(1902)$, pp. 283,284 , contains a solution of a special case of the problem of face traction.

(d) In connection with existence theroms miting to the elastir equations, reference should be made to the wrolk of Italian elisticians, as Sonisiana, Lauricella, anl TeDone. 